\title{
Untersuchungen über die Erfassung von Waldflächen und deren Veränderungen mit Hilfe der Satellitenfernerkundung und segmentbasierter Klassifikation
}

Am Beispiel des Untersuchungsgebietes "Pyeong-Chang" in Korea

Dissertation

zur Erlangung des Doktorgrades der Fakultät für

Forstwissenschaften und Waldökologie der

Georg-August-Universität Göttingen

\author{
vorgelegt von \\ HyunKook Cho \\ geboren in Kangnung / Korea
}

Göttingen im Juni 2002 
D7

1. Berichterstatter: Prof. Dr. A. Akça

2. Berichterstatter: Prof. Dr. J. Saborowski

Tag der mündlichen Prüfung: 5. Juli 2002

Diese Arbeit wurde durch die Niedersächsische Staats- und Universitätsbibliothek unter folgender Internet-Adresse veröffentlicht:

http://webdoc.sub.gwdg.de/diss/2002/cho/index.html 


\section{VORWORT}

Die vorliegende Arbeit wurde unter der Leitung von Herrn Professor Dr. A. Akça am Institut für Waldinventur und Waldwachstum (ehemals Forsteinrichtung und Ertragskunde) der Georg-August-Universität durchgeführt. Für seine großzügige Förderung dieser Arbeit aber auch persönliche Unterstützung möchte ich mich besonders bedanken. Ohne seine familiäre Fürsorge und Geduld wäre die Arbeit nicht gelungen. Mein herzlicher Dank gilt auch Herrn Professor Dr. J. Saborowski für freundliche Übernahme des Korreferates und wertvolle kritische Bemerkungen der Arbeit.

Ein herzlicher Dank gebührt auch Herrn Professor Dr. K. von Gadow sowie allen anderen Mitarbeitern und Mitarbeiterinnen des Institutes für die freundliche Atmosphäre und Hilfestellungen. Besonders zu erwähnen sei Herr Dr. A. Mench, der mir jederzeit mit Rat und Tat zur Seite stand und durch eine sorgfältige fachliche Durchsicht bei der Korrektur meiner Arbeit geholfen hat, sowie Herr Ass. d. Fd. H. Fuchs und Herr Dipl. Forstwirt S. Kunth.

Die vorliegende Arbeit wurde im Rahmen eines gemeinsamen Projektes zwischen dem Institut für Waldinventur und Waldwachstum der Georg-August-Universität und dem „Department of Forest Resources“ der Korea-Universität in Seoul durch die DFG und KOSEF finanziert. Dafür möchte ich mich bei diesen Institutionen bedanken. Besonders bei Herrn Professor Dr. Woo-Kyun Lee, dem Projektleiter der koreanischen Seite, bedanke ich mich sehr herzlich für den freundlichen Empfang und die Unterstützung während der terrestrischen Aufnahmen in Korea.

Nicht zuletzt danke ich ganz besonders meinen Eltern, die meine wissenschaftliche Laufbahn mit großem Vertrauen und viel Geduld begleitet haben und durch ständige Ermutigungen und finanzielle Unterstützungen ein Gelingen der Arbeit ermöglicht haben. Einen ganz besonderen Dank schulde ich meiner Frau Sun-Cheon Kim und meinen Kindern Young-Jun und Young-Bin, die auf viele gemeinsame Zeit verzichten mußten und trotzdem mir immer wieder die Kraft gegeben haben, diese Arbeit zum erfolgreichen Abschluß zu bringen. 


\section{INHALT}

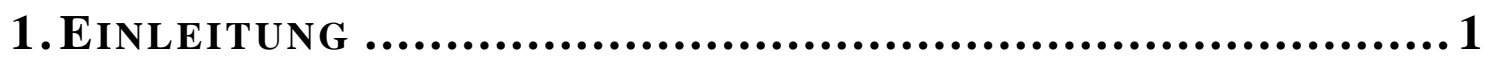

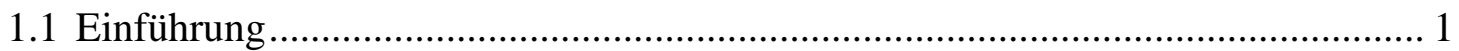

1.2 Entwicklung des koreanischen Waldplanungssystems ...................................... 2

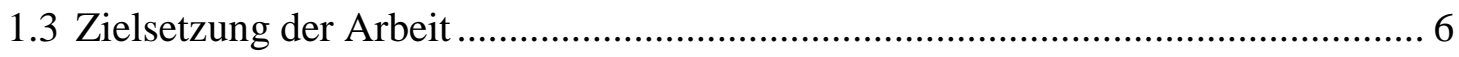

2.STAND DER FORSCHUNG ..................................... 7

2.1 Fernerkundung in der koreanischen Forstwissenschaft...................................... 7

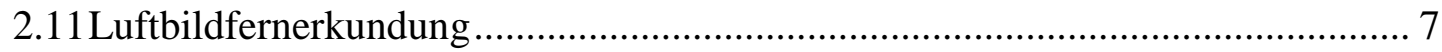

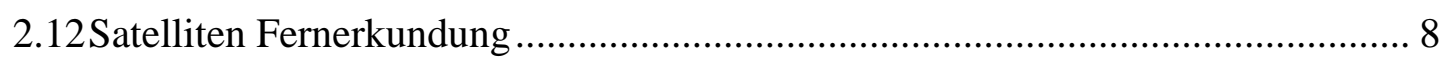

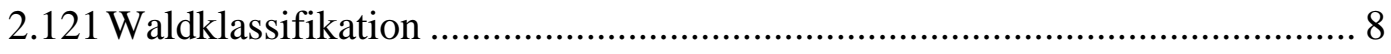

2.122 Erfassung der Waldschäden ................................................................ 9

2.123 Erfassung des nord-koreanischen Waldzustandes ................................. 10

3. UntersuChungSgebiet UND MATERIAL...................... 12

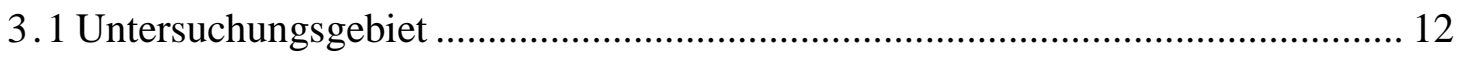

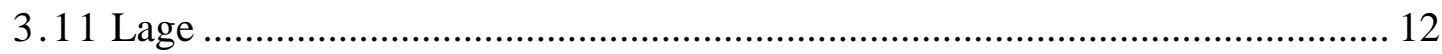

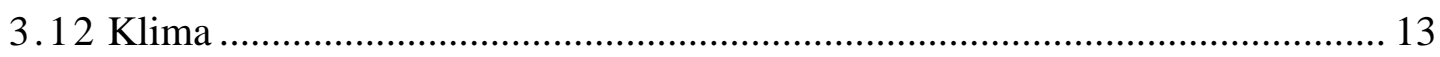

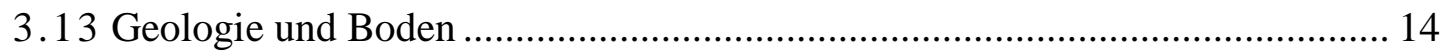

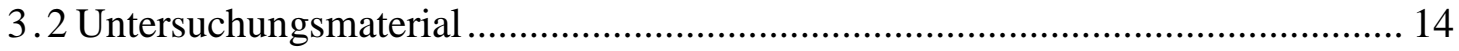

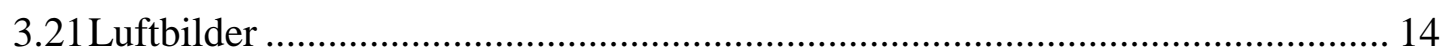

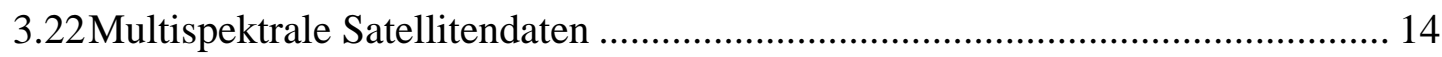

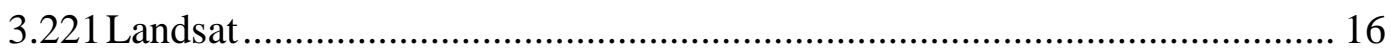

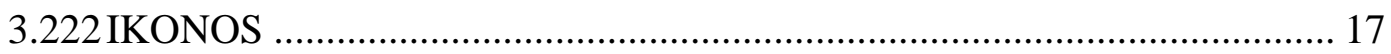

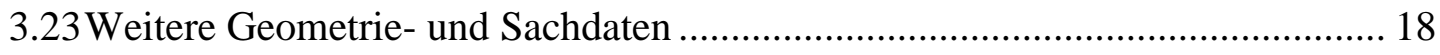

3.231 Topographische Karten und digitales Geländemodell ............................... 18

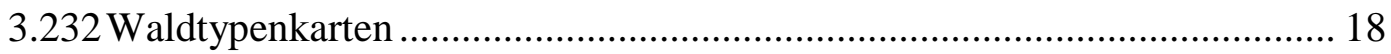

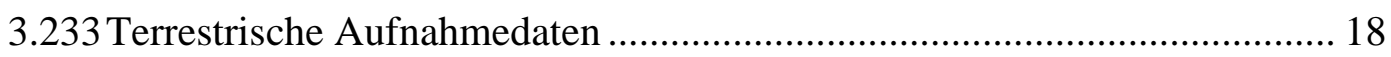

4. METHODE............................................................ 19

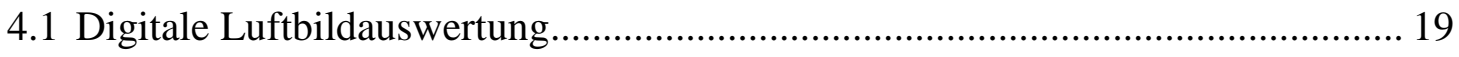

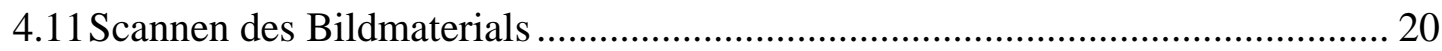

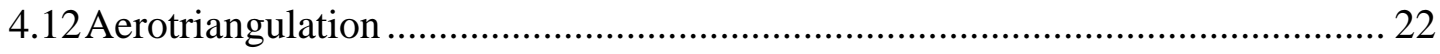

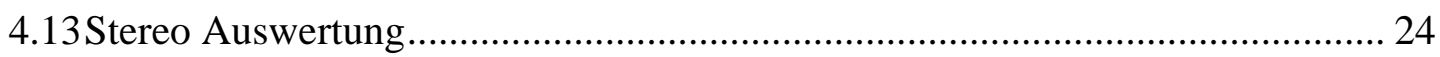

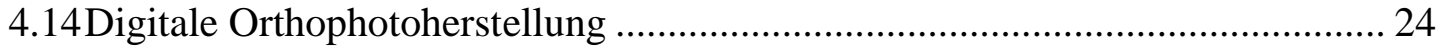

4.2 Vorbereitende Arbeit der Satellitenbildauswertung ........................................... 26

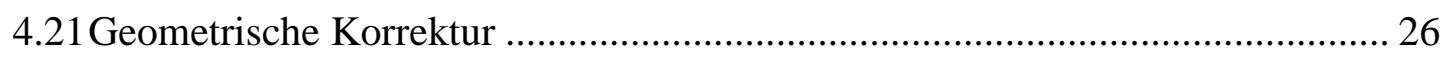

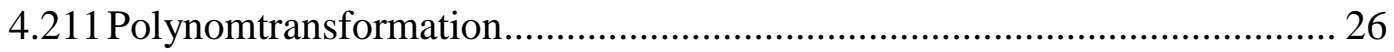




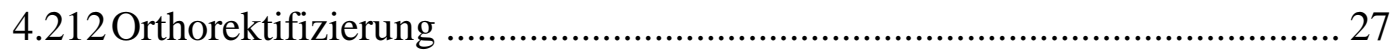

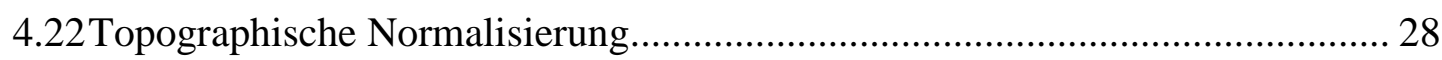

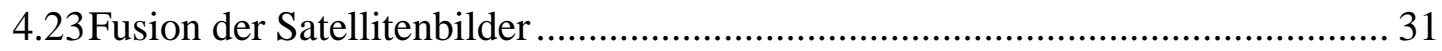

4.3 Klassifikation multispektraler Satellitenbilder ................................................ 34

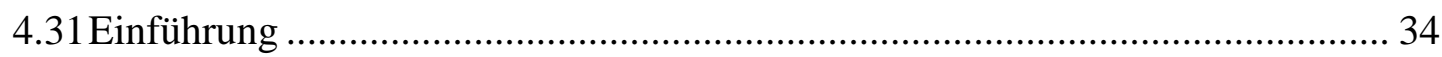

4.32Pixel- und Segment basierte Klassifikation ..................................................... 35

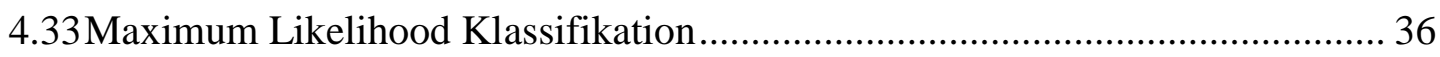

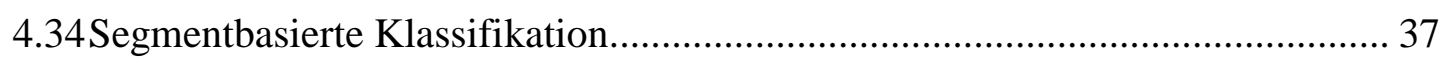

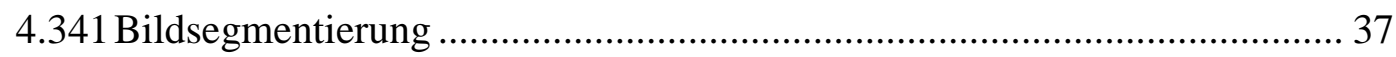

4.342 Multiresolution Segmentation in eCognition ............................................ 39

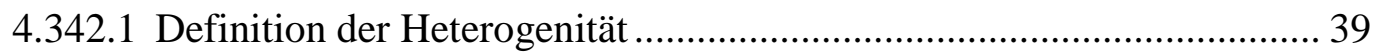

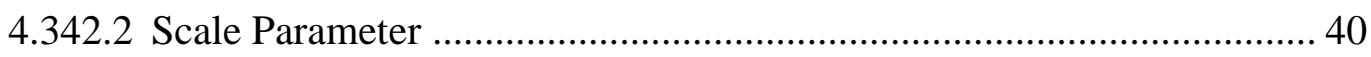

4.342.3 Bestimmung der Parametergröße.......................................................... 41

4.342.4 Hierarchische Netz der Segment........................................................ 43

4.343Zuweisung der Segmente in eine Klasse (Labelling) ................................ 44

4.343.1 Klassenzuweisung nach Mehrheitsprinzip (Mehrheitszuweisung) ....... 44

4.343.2 Segmentbasierte "Maximum Likelihood"-Klassifikation...................... 44

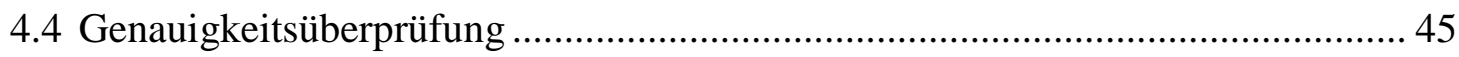

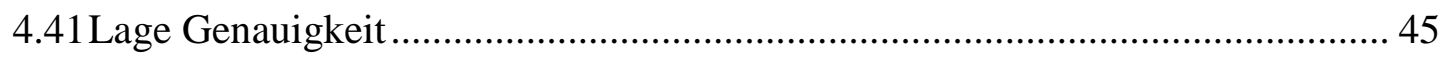

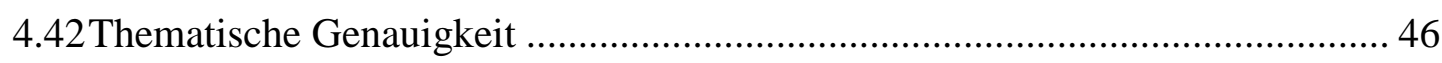

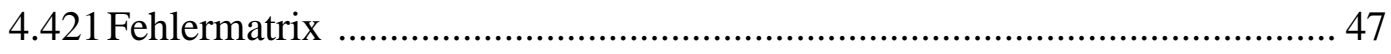

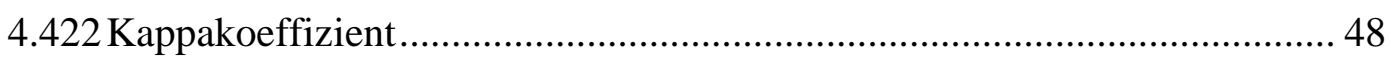

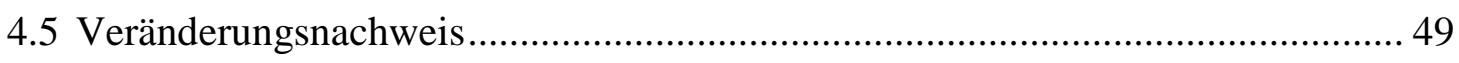

4.51 Veränderungsnachweis mit Vorklassifizierung ............................................ 50

4.52 Veränderungsnachweis mit Nachklassifizierung ........................................... 51

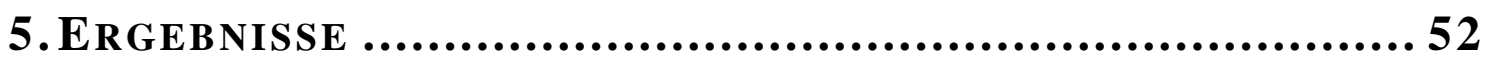

5.1 Herstellung des digitalen Geländemodells ....................................................... 52

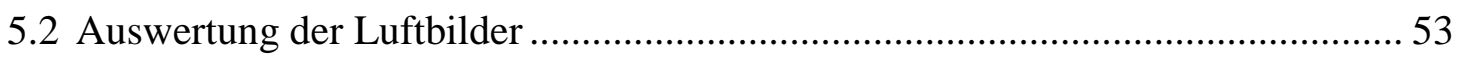

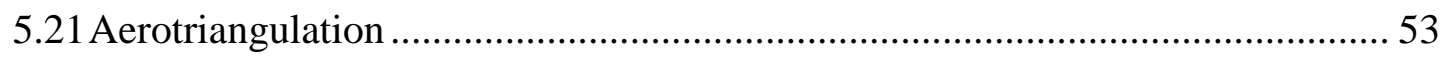

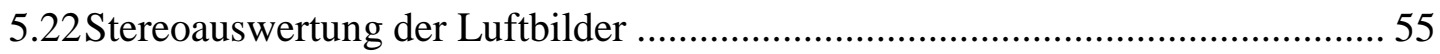

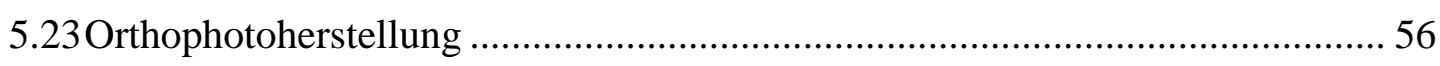

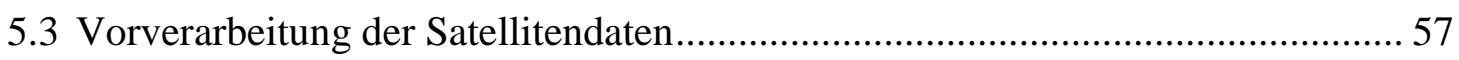

5.31Fusion der multispektralen und panchromatischen Kanäle

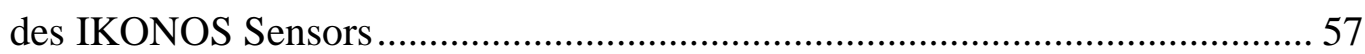

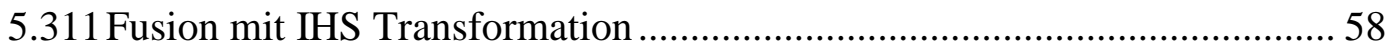

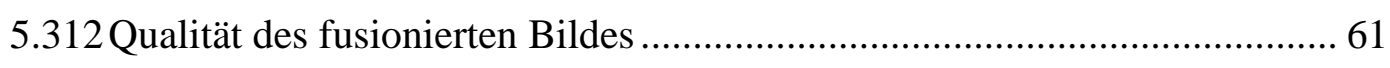

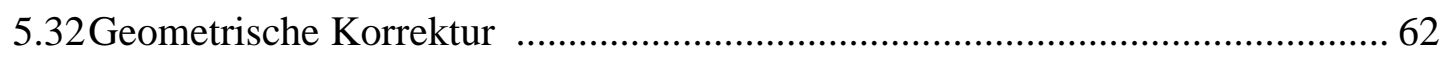

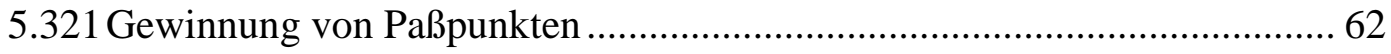

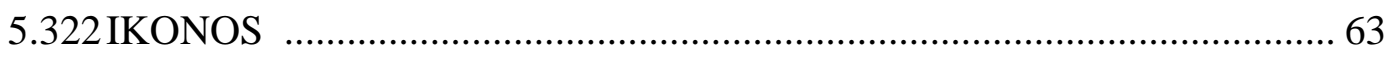

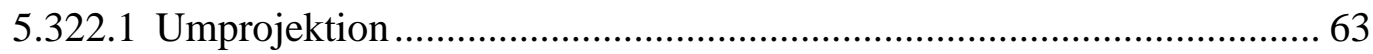




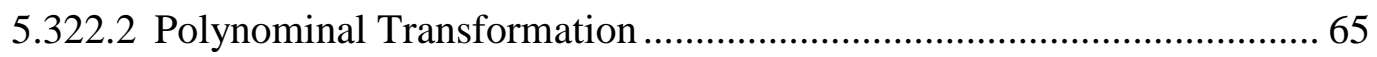

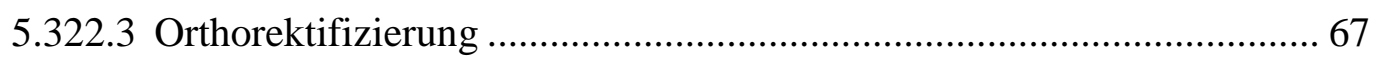

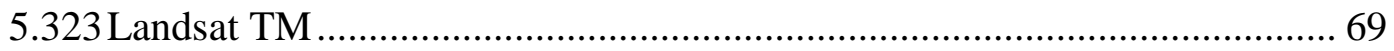

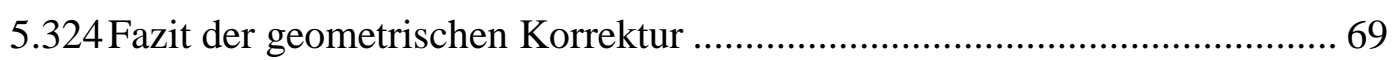

5.33 Topographische Normalisierung der Landsat TM ......................................... 71

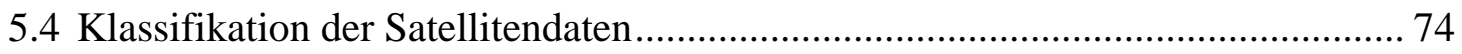

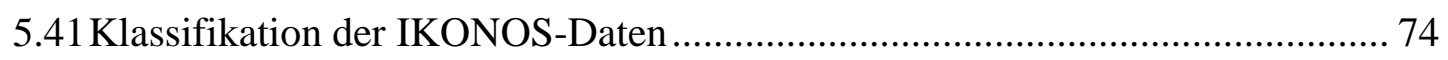

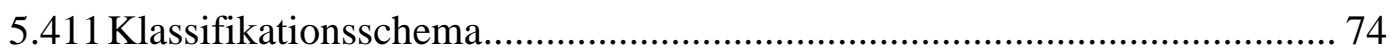

5.412 Pixelbasierte "Maximum Likelihood"-Klassifikation.................................. 75

5.413 Segmentbasierte Klassifikation von IKONOS-Daten............................... 80

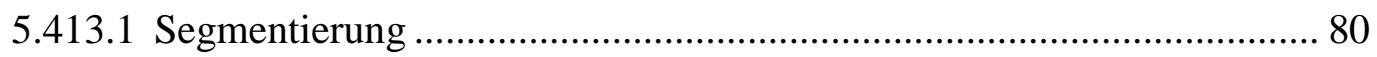

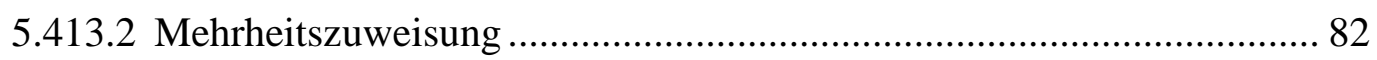

5.413.3 Segmentbasierte "Maximum Likelihood"-Klassifikation ..................... 82

5.414 Beurteilung und Analyse der Klassifikationsergebnisse von IKONOS-

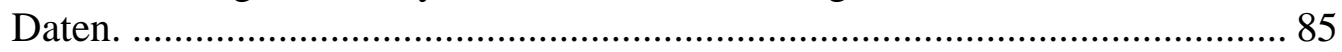

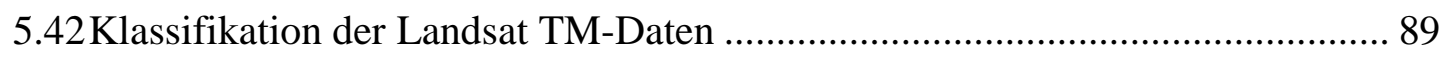

5.421 Pixelbasierte "Maximum Likelihood"-Klassifikation................................ 89

5.422 Segmentbasierte Klassifikation von Landsat TM-Daten ........................... 92

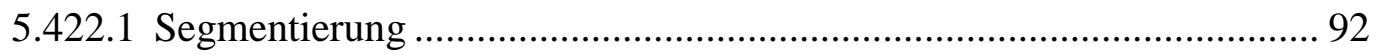

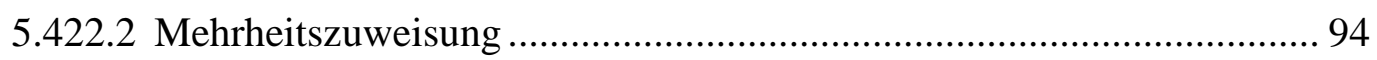

5.422.3 Segmentbasierte "Maximum Likelihood"-Klassifikation....................... 94

5.423 Fazit der Klassifikationsergebnisse von Landsat TM ............................... 95

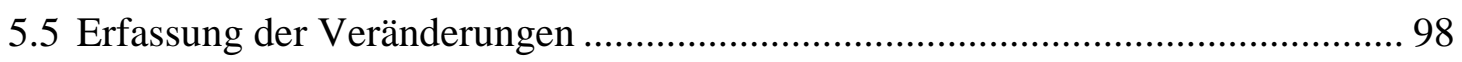

6. DisKUSSION $\ldots . \ldots \ldots \ldots \ldots \ldots \ldots \ldots \ldots \ldots \ldots \ldots \ldots \ldots \ldots \ldots \ldots \ldots \ldots \ldots \ldots \ldots . . \ldots 101$

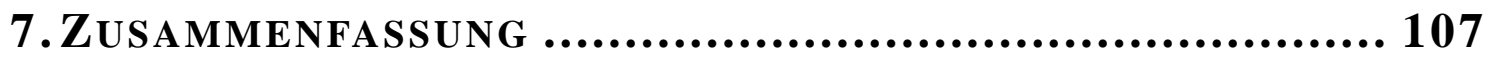

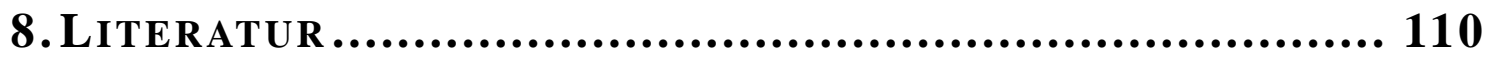

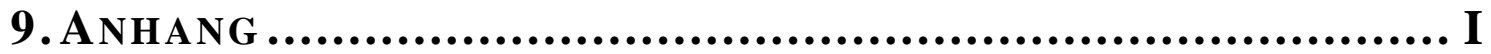




\section{Tabellenverzeichnis}

Tabelle 3.1: $\quad$ Mittlere Temperaturen und Niederschläge von 1991 bis $2000 . \quad 13$

Tabelle 3.2: $\quad$ Technische Daten des verwendeten Luftbildmaterials. $\quad 14$

Tabelle 3.3: Übersicht der wichtigsten Erdbeobachtungssatelliten. 15

Tabelle 3.4: $\quad$ Technische Daten der verwendeten Landsat 5 TM Szene. 16

Tabelle 3.5: $\quad$ Technische Daten der verwendeten IKONOS Szene. 17

Tabelle 4.1: $\quad$ Beispiele für die Dateigröße je Scannauflösung und dementsprechender Bodenauflösung für verschiedene Bildmaßstäbe.

Tabelle 4.2: Aufbau einer Fehlermatrix für eine Klassifikation mit q Klassen.

Tabelle 5.1: $\quad$ Korrelationskoeffizient zwischen den IKONOS Kanälen. $\quad 58$

Tabelle 5.2: $\quad$ Lagefehler der IKONOS Geo Daten nach der Umprojektion, berechnet anhand 20 unabhängiger Kontrollpunkte.

Tabelle 5.3: $\quad$ RMS Fehler nach einer Polynominal-Transformation mit unterschiedlichen Polynomgraden, ermittelt anhand von 27 GCP.

Tabelle 5.4: $\quad$ Lagefehler der IKONOS Geo Daten nach der polynominalen Transformation, berechnet mit 20 unabhängigen Kontrollpunkten.

Tabelle 5.5: $\quad$ RMS Fehler bei der Orthorektifizierung anhand von 27 GCPs.

Tabelle 5.6: Lagefehler der IKONOS Geo Daten nach der Orthorektifizierung, berechnet mit 20 unabhängigen Kontrollpunkten.

Tabelle 5.7: $\quad$ RMS Fehler bei der Transformation.

Tabelle 5.8: $\quad$ Ergebnisse der berechneten Minnaert-Konstanten. 72

Tabelle 5.9: $\quad$ Klassifikationsschema für die vorliegende Untersuchung. $\quad 75$

Tabelle 5.10: Trennbarkeit der Klassen in der IKONOS-Szene. (JefferiesMatusita Best-Minimum Distanz).

Tabelle 5.11: $\quad$ Fehlermatrix für die pixelbasierte "Maximum Likelihood"Klassifikation der IKONOS-Daten.

Tabelle 5.12: Verwendete Parameter für die Segmentierung der IKONOSDaten. 
Tabelle 5.13: Fehlermatrix bei einer Mehrheitszuweisung der IKONOSDaten aufgrund der pixelbasierten „Maximum Likelihood“Klassifikation.

Tabelle 5.14: Trennbarkeit der Klassen in der IKONOS-Szene (JefferiesMatusita Best-Minimum Distanz). Berechnet wurden auf der Segmentbasis.

Tabelle 5.15: Vergleich der Klassifikationsergebnisse der verschiedenen Methoden

Tabelle 5.16: $\quad$ Fehlermatrix für die segmentbasierte „Maximum Likelihood“Klassifikation der IKONOS-Daten.

Tabelle 5.17: Klassifikationsergebnis der IKONOS-Daten zur Kartierung von Waldtypen.

Tabelle 5.18: Flächenbilanz der Klassifikation der IKONOS-Daten

Tabelle 5.19: Trennbarkeit der Klassen auf der Landsat TM-Szene mit der Jefferies-Matusita Distanz.

Tabelle 5.20: $\quad$ Fehlermatrix für die pixelbasierte „Maximum Likelihood“Klassifikation der Landsat TM-Daten.

Tabelle 5.21: Die verwendeten Parameter für die Segmentierung der Landsat TM-Daten.

Tabelle 5.22: Fehlermatrix bei einer Mehrheitszuweisung der Landsat TMDaten aufgrund der pixelbasierten „Maximum Likelihood“Klassifikation.

Tabelle 5.23: $\quad$ Fehlermatrix für die segmentbasierte „Maximum Likelihood“Klassifikation der Landsat TM- Daten.

Tabelle 5.24: Vergleich der Genauigkeit von verschiedenen Klassifikationsmethoden für die Landsat TM-Daten.

Tabelle 5.25: Klassifikationsergebnis der Landsat TM-Daten zur Kartierung von Waldtypen.

Tabelle 5.26: Flächenbilanz der Klassifikation der Landsat TM-Daten.

Tabelle 5.27: Veränderungen der Waldflächen im Untersuchungsgebiet zwischen 1992 und 2000.

Tabelle 5.28: Flächengröße der Wald- bzw. Nichtwald-Klasse und deren Flächenveränderungen. 


\section{Abbildungsverzeichnis}

Abbildung 3.1: $\quad$ Lage des Untersuchungsgebietes. $\quad 12$

Abbildung 4.1: $\quad$ Grundprinzip der Bündelblockausgleichung (KRAUS 1996).

Abbildung 4.2: Zusammenhang zwischen digitalem Oberflächenmodell und Vorlagenbild (KRAUS 1996).

Abbildung 4.3: Schematische Darstellung der Illumination und Sonnenzenit $\left(\theta_{0}\right)$ (modifiziert nach JENSEN 1996).

Abbildung 4.4: Schematische Darstellung der RGB Farbmodelle (links) und der IHS-Doppel Hexcone Modelle (rechts).

Abbildung 4.5: Beispiele der Segmentbildung nach verschiedener Kombination der Parameter. Dargestellt wurden die Ergebnisse anhand einer fusionierten IKONOS Szene.

Abbildung 4.6: $\quad$ Schematische Darstellung einer Objekthierarchie mit drei Segmentierungsebenen (DEFINIENS IMAGING 2001).

Abbildung 5.1: Dreidimensionale Darstellung des Untersuchungsgebietes.

Abbildung 5.2: Verteilung der für die Aerotriangulation verwendeten Paßpunkte und Verknüpfungspunkte.

Abbildung 5.3: Überlagerung der vorhandenen Waldtypenkarte auf dem Luftbild-Orthophoto (a) und den georeferenzierten IKONOSDaten (b).

Abbildung 5.4: Orthophotomosaik aus 35 einzelnen Orthophotos.

Abbildung 5.5: Darstellung des Orthophotos mit Höhenschichtlinien.

Abbildung 5.6: Vergleich der Intensitätskomponente in IHS Farbsystem (a) mit dem panchromatischen Datensatz (b).

Abbildung 5.7: Gegenüberstellung der originalen multispektralen Bilder (links) und der fusionierten Bilder (rechts).

Abbildung 5.8: Verteilung der „Ground Control Points“ (GCP: \#) und der unabhängigen Kontrollpunkte (Independent Control Points: ICP : ð).

Abbildung 5.9: Graphische Darstellung der Lagefehler nach der Umprojektion.

Abbildung 5.10: Graphische Darstellung der RMS Fehler nach der Polynominal Transformation.

Abbildung 5.11: Graphische Darstellung der RMS Fehler nach der Orthorektifizierung. 
Abbildung 5.12: Vergleich des orthorektifizierten Satellitenbild Landsat TM (a), der fusionierten IKONOS Szene (b) und des LuftbildOrthophotos (c).

Abbildung 5.13: Berechnung der Minnaert-Konstante. Die Steigung der Regressionslinie mit umgekehrtem Vorzeichen ist die Minnaert Konstante.

Abbildung 5.14: Vergleich zwischen den Originaldaten (a) und den Korrekturergebnisse (b) nach der Minnaert-Korrektur für die Landsat TM-Daten.

Abbildung 5.15: Mittlere Spektralsignaturen der fusionierten IKONOS-Szene für ausgewählte Trainingsflächen verschiedener Landbedeckungsklassen(a) bzw. verschiedener Waldklassen(b)

Abbildung 5.16: Segmtierungsergebnisse der IKONOS-Daten.

Abbildung 5.17: Mittlere Standardabweichungen der IKONOS-Szene auf der Segmentbasis.

Abbildung 5.18: Waldtypenkarte des Untersuchungsgebietes erstellt aus der Klassifikation der IKONOS-Daten von 2000.

Abbildung 5.19: Mittlere Spektralsignaturen verschiedener Waldklassen anhand ausgewählter Trainingsflächen auf der Landsat TM-Szene.

Abbildung 5.20: Die Streuungsellipsen ( \pm zweifache Standardabweichung) verschiedener Waldtypen im zweidimensionalen Merkmalsraum.

Abbildung 5.21: Segmentierungsergebnisse der Landsat TM-Daten

Abbildung 5.22: Waldtypenkartierung des Untersuchungsgebietes, hergeleitet aus der Klassifikation der Landsat TM-Daten aus dem Jahr 1992.

Abbildung 5.23: Veränderungen von Wald und Nichtwald im Zeitraum 1992 bis 2000

Abbildung 6.1: Vergleich der beiden Datensätze Landsat TM (links) und IKONOS (rechts) in einem kleinen Ausschnitt von ca. $1 \mathrm{~km} \mathrm{x}$ $1 \mathrm{~km}$ Größe. (a) Original-Daten mit dem Grenzen der Segmente, (b) Pixelbasierte Klassifikation (c) Segmentbasierte Klassifikation

Abbildung 6.2: Beispiel einer GIS-Operationen mit Ergebnis einer segmentbasierten Klassifikation. 


\section{Einleitung}

\subsection{Einführung}

Unter dem Begriff „Fernerkundung“ versteht man die „Aufnahme, Messung bzw. Erkundung gewisser Eigenschaften eines Objektes aus der Distanz, d.h. ohne das Aufnahmeinstrument und Objekt miteinander in Kontakt stehen " (AKÇA, 2001). Die Anwendung der Fernerkundung in der Forst- bzw. Waldwirtschaft zielt unter anderem auf die Gewinnung von planungsrelevanten Informationen. Der Fernerkundung ist dann den Vorzug $\mathrm{zu}$ geben, wenn sie gewisse Vorteile gegenüber anderen Informationsquellen bietet, nämlich die Informationen zeitlich günstiger oder preiswerter oder mit besserer Qualität liefern.

Terrestrische Aufnahmemethoden ermöglichen zwar eine Gewinnung von detaillierten Informationen, sie sind jedoch großräumig zeit- und kostenaufwendig. Moderne terrestrische Stichprobenverfahren bieten inzwischen in Verbindung mit einem geographischen Informationssystem (GIS) und der Fernerkundung die Möglichkeit, raumbezogene Informationen mit einem erheblich geringeren Aufwand und höherer Qualität großräumig zu erfassen, wie zahlreiche Untersuchungen belegt haben.

In den letzten Jahren hat die Anzahl von verfügbaren SatellitenFernerkundungssystemen stark zugenommen. Vor allem wurde eine neue Generation von hochauflösenden optischen Satellitensystemen der zivilen Erdbeobachtung angekündigt und in Betrieb genommen. Sie verfügen neben einer hohen räumlichen und spektralen Auflösung über eine relativ hohe zeitliche Auflösung. Darüber hinaus bieten sie die Möglichkeit von Stereoaufnahmen an. Mit der Inbetriebnahme der neuen Generation moderner Satellitensysteme wie z.B. IKONOS hat die Anwendung von Satellitendaten in vielen Bereichen der Ökologie der Erdoberfläche an Attraktivität gewonnen.

Eine erhöhte geometrische Auflösung bietet insbesondere in kleinflächig bewirtschafteten Gebieten bessere Interpretationsmöglichkeiten. Auf der anderen Seite erschwert sie eine rechnergestützte digitale Klassifikation, weil die hohe geometrische Auflösung die Varianz der Pixelwerte innerhalb einer Klasse erhöht. Darüber hinaus stellt der sogenannte „Salz und Pfeffer Effekt“ (sehr kleinflächige 
Klassifizierungsergebnisse) bei einer herkömmlichen pixelbasierten Klassifikation ein Problem für die integrierte Nutzung in einem GIS dar.

Zur Lösung dieses Problems bietet sich die sogenannte segmentbasierte Klassifikation an. Segmentierung ist ein Verfahren, mit dem ein Bildraum in mehrere Regionen zerlegt wird, die sich untereinander nicht überlappen und homogen sind (PAL et al., 2000). Nach der Segmentierung können anstelle von Pixeln die einzeln Segmente klassifiziert werden.

Gegenüber der pixelbasierten zur der segmentbasierten Klassifikation sind unter anderem folgende Vorteile zu erwähnen.

- Ein Segment als eine Gruppe von Pixeln mit ähnlichen Spektralwerten kann sicherer klassifiziert werden als einzelne Pixel.

- Neue Merkmale, z.B. die Form oder Nachbarschaftsbeziehungen der Segmente können für die Klassifikation herangezogen werden. Darüber hinaus können durch die hierarchischen Beziehungen zwischen Ebenen von unterschiedlich großen Segmenten wertvolle Informationen zur Klassifikation gewonnen werden.

- Die segmentbasierte Klassifikation ist weniger rechenaufwendig als die pixelbasierte Klassifikation, weil durch die Segmentierung weniger Klassifikationseinheiten als Pixel entstehen.

- Die Ergebnisse einer segmentbasierten Klassifikation können leichter in ein GIS integriert werden, weil es keinen „Salz und Pfeffer Effekt“ gibt.

\subsection{Entwicklung des koreanischen Waldplanungssystems}

Bis zum 19-ten Jahrhundert waren die koreanischen Wälder meist vielfältige, strukturreiche Altwuchswälder. Diese Wälder wurden während der japanischen Besatzungszeiten (1910-1945) und des koreanischen Krieges (1950-1953) durch Übernutzung und illegale Nutzung für die Brennholz- und Baumaterialgewinnung sowie durch kriegerische Handlungen fast vollständig zerstört. Das durchschnittliche Vorratsvolumen im Jahr 1960 lag nur noch bei 10,6 $\mathrm{m}^{3}$ pro Hektar. 
Erst 1961 entstand das erste Waldgesetz Koreas. Damit wurden verschiedene Vorschriften, die aus japanischen Besatzungszeiten entlehnend angewendet und nicht kompetent waren, außer Kraft gesetzt und es wurde eine systematische Gesetzgebung geschaffen. Das Waldgesetz wurde im Laufe der Zeit mehrmals überarbeitet und am 01. 01. 2002 ist ein neues Waldgrundgesetz in Kraft getretnen. Das Gesetz schreibt vor, alle 10 Jahre eine Forstrahmenplanung auf nationaler Ebene aufzustellen, um die Wälder zu schützen, zu vermehren und eine nachhaltige Bewirtschaftung der Waldressourcen zu ermöglichen. Die Landesregierungen sollten anschließend auf Basis der nationalen Rahmenplanung regionale Forstplanungen erstellen.

Im folgenden soll ein kurzer Überblick über die Entwicklung des koreanischen Waldplanungssystems gegeben werden.

\section{Erster 10-jähriger Forstentwicklungsplan: 1973 - 1978}

Als ein Wendepunkt in der koreanischen Forstwirtschaft wird die Erstellung des "Ersten 10-jährigen Forstentwicklungsplanes" aus dem Jahr 1973 angesehen, der in erster Linie die Wiederaufforstung zerstörter Wälder vorsah. Etwa eine Million Hektar zerstörter Wälder sollte innerhalb dieses Planungszeitraumes aufgeforstet werden.

Eine bemerkenswerte Aktion in dieser Zeit war die Erklärung der "Nationalen Pflanzperiode", die vom 21. März bis zum 20. April dauerte und den 5. April als besten Pflanztag erklärte. Die gesamte Bevölkerung Koreas hat in dieser Zeit am Wiederaufforstungsprogramm teilgenommen. Darüber hinaus wurde die erste Woche im November als "Baumpflegewoche" erklärt, in der dann eine ganze Reihe von Pflegemaßnahmen durchgeführt wurde. Während der Periode des ersten Forstentwicklungsplanes wurden durch Einschränkungen des Zugangs zu den Waldbeständen und Gründung von speziellen Brennholzbeständen erhebliche Fortschritte im Hinblick auf einen Waldschutz in die Wege geleitet.

Durch die aktive Teilnahme der Bevölkerung und die Förderung der Regierung wurde das Planungsziel des "Ersten 10-jährigen Forstentwicklungsplanes" bereits nach 6 Jahren mit einer Wiederaufforstungsfläche von 1.08 Million Hektar erreicht und erfolgreich abgeschlossen. Die Hauptbaumarten, die in diesem Zeitraum gepflanzt wurden, waren Pappel, Lärche, koreanische weiße Kiefer und Kastanie. 


\section{Zweiter 10-jähriger Forstentwicklungsplan: 1979 - 1987}

Das Hauptziel des "Zweiten 10-jährigen Forstentwicklungsplanes" war die Gründung großflächiger Wirtschaftwälder zur Deckung des langfristigen Holzbedarfs. Zur erfolgreichen Umsetzung des Planes initiierte die Regierung verschiedene Entwicklungsprogramme, wie z.B. die Erweiterung des Wiederaufforstungsplanes, die Verstärkung von Waldschutzmaßnahmen, eine Erweiterung der Waldentwicklungsfonds für die Unterstützung von Privatwäldern, eine Vergrößerung des Nationalwaldes und die Einführung eines Walderhaltungsprogramms zur Verbesserung der öffentlichen Waldnutzung.

Eine Hauptleistung während dieser Periode war die Gründung von 80 großflächigen Forstbetrieben, wobei 325,000 Hektar Waldfläche wieder aufgeforstet wurden. Kontinuierliche Pflegemaßnahmen wurden in Naturwäldern und Jungplantagen durchgeführt. Weiterhin wurden verschiedene Waldschutzmaßnahmen gegen Waldbrände, Waldkrankheiten und Insektenschäden durchgeführt. Trotz zahlreicher Bemühungen wurde bis heute kein geeignetes Mittel gegen die Gallmücke entwickelt, die ein sehr kritischer Feind der Kiefernwälder, insbesondere der Pinus densiflora Bestände, ist. Darüber hinaus wurde eine Waldarbeiterschule eingerichtet, um die Maschineneinsatzquote bei Waldarbeiten zu erhöhen und die Waldarbeiter entsprechend auszubilden. Zusätzlich wurden auf der Grundlage eines neuen Walderschließungsplans $786 \mathrm{~km}$ Waldwege neu gebaut.

\section{Dritter Forstentwicklungsplan (Waldressourcen Erweiterungsplan): 1988 - 1997}

Auf der Basis der erfolgreichen Wiederaufforstung im Rahmen der Ersten und Zweiten Forstentwicklungspläne wurde der Dritte Forstentwicklungsplan aufgelegt. Das Hauptziel war dabei eine Maximierung der Wohlfahrtswirkungen der Wälder durch die Harmonisierung der wirtschaftlichen und übrigen öffentlichen Interessen. Der Plan sah vor eine vernünftige, geregelte und nachhaltige Nutzung der Wälder, die Sicherung der forstwirtschaftlichen Grundlagen und eine Verbesserung der Umweltqualität zu gewährleisten.

Die Hauptaktivitäten in dieser Planungsperiode waren die Gründung von 320,000 Hektar von Wirtschaftwäldern, die Durchführung von Pflegemaßnamen auf 3,030,000 Hektar Waldfläche, die Schaffung einer forstwirtschaftlichen Infrastruktur durch 
Walderschließung sowie die Ausbildung der Waldarbeiter. Weiterhin wurde ein großer Beitrag zur öffentlichen Nutzung der Wälder geleistet, wie z. B. die Einrichtung von Sportplätzen oder Erholungsplätzen in naturgeprägten Wäldern.

Darüber hinaus wurden verschiedene Naturschutzmaßnahmen durchgeführt. Für eine gesicherte Wasserversorgung wurden 5 bedeutende Flüsse und ihre Umgebungen unter einem Sonderprogramm intensiv kontrolliert. Darüber hinaus wurden wilde Vögel und andere Tiere in das Schutzprogramm einbezogen. Zusätzlich wurde im Jahr 1995 ein Walddorfentwicklungsplan initiiert, um den Lebensstandard der Dorfbewohner, die isoliert im Gebirgsdörfern wohnen, zu verbessern und für sie neue Einkommensquellen zu erschießen. Dazu hat auch die wissenschaftliche Forschung und die Anwendung von modernen Technologien wie z. B. die Entwicklung von Krebsmedikamenten aus Eiben oder die Herstellung von biologisch abbaubaren Holzkunststoffen beigetragen.

\section{Vierter Forstentwicklungsplan: 1998 - 2007}

Die ersten drei Forstentwicklungspläne waren in erster Linie unter der Leitung der Regierung durchgeführte Aufforstungs- und Begrünungspläne. Im vierten Forstentwicklungsplan ist hauptsächlich die Förderung der einzelnen Waldbesitzer vorgesehen. Sie sollen dabei zur Harmonisierung der vielfältigen Anforderungen der Gesellschaft an die Wälder ihre Entscheidungen selbst treffen.

Der zur Zeit laufende "Vierte Forstentwicklungsplan" hat als Hauptziel die "Schaffung einer Basis für die nachhaltige Waldbewirtschaftung". Diese Zielsetzung soll durch eine Förderung der Herstellung hochwertiger Forstprodukte, Stärkung einer wettbewerbsfähigen Forstindustrie und der Förderung einer gesunden Forstumwelt erreicht werden.

Zur umweltfreundlichen Bewirtschaftung der Wälder soll dabei die Forstverwaltung effizienter gestaltet werden. Die Wirtschaftwälder sollen zur nachhaltigen Deckung des nationalen Holzbedarfs weiterhin flächenmäßig vergrößert werden. Die Intensive Ausbildung der Förster soll fortgesetzt werden.

Zum Schutz der Waldökosysteme sollen darüber hinaus Waldschutzmaßnahmen verstärkt in Angriff genommen werden. Die Wettbewerbsfähigkeit der Forst- und Holzindustrie und des Holzmarktsystems soll gefördert bzw. verbessert werden. 
Weiterhin wird die Wiedervereinigung von Korea angestrebt und die internationale Zusammenarbeit im forstwirtschaftlichen Bereich verstärkt.

\subsection{Zielsetzung der Arbeit}

Die Zielsetzung dieser Arbeit ist vor diesem Hintergrund, einen Beitrag zur Verwendung von hoch- und höchstauflösenden Satellitendaten, Landsat TM und IKONOS, für forstliche Zwecke zu leisten. Die Untersuchung wurde in einem nordöstlichen Waldgebiet in Südkorea durchgeführt. Sowohl eine pixel- als auch eine segmentbasierte Klassifikation der beiden Satellitendaten sollen im Hinblick auf ihre geometrischen und thematischen Klassifizierungsgenauigkeiten überprüft und die Ergebnisse miteinander verglichen werden. Darüber hinaus sollen beide Klassifizierungsverfahren auch im Hinblick auf Ihre Eignung als Mittel zum Veränderungsnachweis getestet werden. 


\section{Stand der Forschung}

\subsection{Fernerkundung in der koreanischen Forstwissenschaft}

\subsection{Luftbildfernerkundung}

Der Einsatz von Luftbildern in der Forstwirtschaft Koreas begann Anfang der Siebziger Jahre. Im Rahmen der „Ersten Nationalwaldinventur“ (1971-1975) wurden die erstmalig Luftbilder für die Waldgebiete flächendeckend aufgenommen(KFS: KOREA FOREST SERVICE, 2001). Dieses Luftbildmaterial wurde unter zusätzlicher Verwendung von terrestrischen Aufnahmen zur Herstellung von Waldtypenkarten, Regressionsmodellen für die bestandesweise Holzvorratschätzung und für die Herstellung einer LuftbildMassentafel eingesetzt. Die Ergebnisse sind in den nationalen Waldstatistiken integriert und bilden die Grundlage für die Überprüfung der abgelaufenen Planung und der Erstellung der zukünftigen Planung, sowie für politische Entscheidungen. Die Nationalwaldinventur wird in einer 10-jährigen Periode wiederholt, zur Zeit läuft die vierte Inventur.

Die Luftbilder wurden ebenfalls für verschiedene Forschungsprojekte herangezogen. YUN und CHA (1995) untersuchten die Bestandesstrukturen der koreanischen Weißkiefer-Bestände (Pinus koraiensis) mit Hilfe der Luftbildauswertung. In dieser Untersuchung wurden Bestandesparameter, wie z.B. Dichte, Volumen der Bestände, der mittlere Durchmesser und die Durchmesserstreuung unter Verwendung einer Weibull Verteilungsfunktion auf Grund von photogrammetrischen Auswertungen und terrestrischen Aufnahmen geschätzt. Der Vergleich mit den terrestrischen Aufnahmeergebnissen zeigte, daß die aus dem Luftbild geschätzten Werte der Bestandesstrukturparameter und die terrestrisch hergeleiteten Werte auf einem 99\% Signifikanzniveau keine Unterschiede aufwiesen.

Für die Erfassung der Landnutzungsänderung und Volumenschätzung haben $\mathrm{OH}$ und $\operatorname{KIM}(1990)$ eine Luftbildzeitreihe herangezogen. Durch visuelle Interpretation von Schwarz-Weiß Luftbildern aus den Jahren 1979 und 1988 wurden die Waldbestände getrennt nach einzelnen Baumarten kartiert. Anschließend wurden die Volumina mit Hilfe eines Regressionsschätzers geschätzt und der Zuwachs hergeleitet. 


\subsection{Satelliten Fernerkundung}

1984 würde in Korea die „Korean Society of Remote Sensing (KSRS)“, die als ein Eckstein in der koreanischen Fernerkundungsgeschichte angesehen wird, gegründet. KSRS hat die Techniken für eine digitale Bildverarbeitung und ihre Anwendungsmöglichkeiten in verschiedenen Bereichen des Landes eingeführt. Mitte der neunziger Jahre wurde das „National Geographic Information System (NGIS)“, in dem die Fernerkundung als wichtige Informationsquelle erneut anerkannt wurde, initialisiert. Besonders nennenswert ist die Entwicklung des koreanischen MehrzweckSatelliten KOMPSAT, der einen Erdbeobachtungssensor besitzt. Es wird erwartet, daß die Satelliten-Fernerkundung für forstliche Fragestellungen auch in Korea immer häufiger zur Anwendung kommt.

\subsection{Waldklassifikation}

Zur Klassifizierung der Waldtypen und Charakterisierung der Waldverteilung verwendete KIM (1991) Landsat TM Daten und digitale Geländemodelle. Mit einer nicht überwachten Klassifizierungsmethode wurden im Untersuchungsgebiet Gumo-san in Korea sechs verschiedene Landbedeckungsarten einschließlich drei Waldtypen Nadelwald, Laubwald und Mischwald - klassifiziert und kartiert. Die Klassifikationsergebnisse wurden mit den Ergebnissen einer Interpretation der SchwarzWeiß Luftbilder verifiziert. Dabei wurde festgestellt, daß der Flächenanteil von Mischwäldern untergeschätzt während derjenige von reinen Nadel- und Laubwäldern übergeschätzt wurde. Zur Charakterisierung der Waldverteilung im Zusammenhang mit der Topographie wurden einige Parameter wie Bestandeslagehöhe, Aspekte und Niederschlag als Akkumulierungswerte aus dem DGM hergeleitet. Diese Werte zeigen eine hohe Korrelation mit dem Waldtypen. Die Einbeziehung dieser Parameter bei einer Klassifizierung von Waldtypen würde somit zu verbesserten Ergebnissen führen.

LEE et. al, (1999) klassifizierten die Landoberfläche der koreanischen Halbinsel mit Hilfe einer Bildkomposition aus monatlichen NOAA/AVHRR NDVI Aufnahmen von April bis November 1998. Mit einer visuellen Überprüfung der Klassifikation konnten befriedigende Ergebnisse bei allen Landbedeckungsklassen festgestellt werden. Die Waldtypenklassifizierung war dabei jedoch nicht befriedigend. Eine statistische Überprüfung der Genauigkeit wurde nicht durchgeführt. 


\subsection{Erfassung der Waldschäden}

Bei mehreren Untersuchungen wurde die Möglichkeit überprüft, Waldschäden wie z.B. durch Waldbrände oder Insekten mit Hilfe der Satelliten-Fernerkundung zu erfassen. WON und IM (2001) versuchten mit Landsat 7 ETM+ Daten, die einen Monat nach einem Waldbrand aufgenommen waren, die Waldbrandflächen nach einem 3-stufigen Schadensgrad zu klassifizieren. Dabei wurden die „Brightness-,, „Greenness-,, und „Wetness-,,Komponente der „Tasseled Cap“ Transformation zur Klassifizierung herangezogen und die Ergebnisse mit den Klassifikationsergebnissen der IHS transformierten Daten (Band 7,4,1) verglichen. Die Ergebnisse wurden mit 200 terrestrisch aufgenommenen Referenz-Daten verifiziert. Die K-Hat Statistik weißt einen Wert von 0.76 bei der „Tasseled Cap“ Transformation und von 0.67 für die IHS Transformation auf.

MA et. al, (1999) erfaßten die Waldbrandtflächen mit Landsat TM Daten und schätzten den Volumenverlust. In dieser Studie wurden mit Hilfe eines GIS die Stichproben aus den gleichen Waldtypen und topographischen Bedingungen wie die Brandflächen ausgewählt. Durch terrestrische Aufnahmen wurden zuerst das Volumen der Stichprobeflächen und anschließend die durch Waldbrand entstandenen Volumenverluste hergeleitet.

Jo et. al, (2000) versuchten die Waldbrandgefahr mit Hilfe der Satellitenfernerkundung zu modellieren. Als Eingangsparameter für die verwendeten logistischen Regressionsmodelle dienten dabei u. a. Waldtypen, gewonnen durch eine Klassifikation von Landsat TM Daten, Klimadaten und topographische Parameter. Sie prognostisierten sowohl die Waldbrandgefahr als auch die Richtung und Geschwindigkeit der Waldbrände.

Fernerkundung wurde auch für die Erfassung und Analyse der verschiedenen Insektenschäden eingesetzt. KIM et. al, (2001) haben für die Erfassung der Schadensflächen multitemporale Landsat TM Daten verwendet. Die temporalen und räumlichen Verhaltensweisen der Schäden wurden zusammen mit Klimadaten analysiert. Dabei konnte ein enger Zusammenhang zwischen den Insektenschäden, der Windgeschwindigkeit in den Monaten Mai und Juni sowie dem Niederschlag im Monat Juli festgestellt werden.

Zur Erfassung der Insektenschäden und deren räumlichen Verteilung hat $\mathrm{OH}$ (1998) 
multitemporale Landsat TM-Daten mit einer digitalen Waldtypenkarte kombiniert. In dieser Untersuchung wurden zunächst die Kiefernbestände aus der digitalen Waldtypenkarte ausgeschnitten, weil die in dem Untersuchungsgebiet aufgetretende Gallmücke(Thecodiplosis japonisis) nur die Kiefernbestände befällt. Nach einer Normalisierung der topographischen Einflüsse wurden die Kiefernbestände nach 4 Stufen klassifiziert. Durch Analyse des Zusammenhangs zwischen dem Schadensvorkommen und der Exposition konnte festgestellt werden, daß Bestände am Nordhang schwerere Schäden als am Südhang aufweisen.

\subsection{Erfassung des nord-koreanischen Waldzustandes}

In letzter Zeit wurde dem Waldzustand in Nord-Korea großes Interesse beigemessen. Weil es keine zuverlässigen Informationen über den aktuellen Waldzustand Nordkoreas gibt und kein Zugang zum nordkoreanischen Wald möglich ist, wird die SatellitenFernerkundung als einzige alternative Informationsquelle angesehen.

LEE et. al, (1998) untersuchten die Waldflächen und den Holzvorrat in Nordkorea mit Hilfe von Satelliten-Fernerkundung. Insgesamt 13 Landsat TM-Szenen der Jahre 1991 und 1994 wurde mit einer kombinierten Methode aus „ISODATA“ und „Maximum Likelihood“ die Landbedeckungsart klassifiziert. „Ground Truth“ Daten für die Klassifizierung wurden aus bereits verfügbaren Karten gewonnen. Gebiete, die als Wald klassifiziert worden sind, wurden mit NDVI in 3 Dichteklassen weiter differenziert. Eine Zwei-Stufige-Stichproben-Methode wurde für die Schätzung des Volumens herangezogen. Die gesamten Waldflächen Nordkoreas betrugen ca. 8,45 Millionen Hektar. Dies ist ca. 1,32 Millionen Hektar weniger als die letzte offizielle Angabe Nordkoreas aus dem Jahre 1970. Der Gesamtvorrat wurde auf 340 Millionen Kubikmeter, d.h. 40,6 Kubikmeter pro Hektar, geschätzt.

LEE und KIM (2000) versuchten die Veränderung der Waldflächen Nordkoreas und der Kronenzustände in den letzten 30 Jahren mit Hilfe von multisensor- und multitemporalen Satellitendaten zu erfassen. Landsat MSS, SPOT und KOMPSAT Daten wurden dabei eingesetzt. Für die Erfassung der Waldflächenänderung wurden die Waldgrenzen aus einer im Jahr 1981 veröffentlichten Karte digitalisiert und mit eine KOMPSAT Daten aus dem Jahre 2000 maskiert. Durch eine visuelle Interpretation und nicht überwachte Klassifikation wurden die kahlgeschlagenen Waldflächen aus dem 
maskierten Bild ausgeschnitten. Durch einen Vergleich der NDVI Werte konnte festgestellt werden, daß sich die Bedeckungsgrade der Wälder in der Nähe von Großstädten verschlechtert, dagegen in weiter von Großstädten entfernten Gebieten verbessert haben.

KIM und PARK (2000) analysierten die Waldfragmentierung in Nordkorea in dem Zeitraum zwischen 1979 und 1998. In dieser Untersuchung wurde zunächst die Landnutzung als Wald- und Nicht-Wald klassifiziert. Über die Waldklasse wurden einige Landscape-Indizes im Bezug auf Dichte, Größe, und Form gerechnet. Die Ergebnisse zeigten, daß sich die Gesamtwaldfläche in dem Untersuchungszeitraum signifikant reduziert hat und die einzelnen Waldflächen kleiner geworden sind. 


\section{Untersuchungsgebiet und Material}

\subsection{Untersuchungsgebiet}

\subsection{Lage}

Die koreanische Halbinsel liegt am östlichen Ende des asiatischen Kontinents zwischen China und Japan und grenzt landseitig im Norden an China und Rußland. Ihre Gesamtfläche beträgt $212300 \mathrm{~km}^{2}$, davon entfallen $99300 \mathrm{~km}^{2}$ auf die Republik Korea (Südkorea).

Das Untersuchungsgebiet "PyeongChang" befindet sich in der Mitte der koreanischen Provinz "Kangwon-Do", die im östlichen Teil von Südkorea liegt. Das Gebiet umfasst eine Fläche von ca. $1500 \mathrm{~km}^{2}$ und liegt zwischen den geographischen Koordinaten $128^{\circ} 14^{\prime}$ und $128^{\circ} 46^{\prime}$ östliche Länge sowie zwischen $37^{\circ} 16^{\prime}$ und $37^{\circ} 49^{\prime}$ nördliche Breite. Da die finanziellen Mittel innerhalb des Forschungsprojektes begrenzt und lediglich die Möglichkeiten und eventuelle Beschränkungen einer neuen Untersuchungsmethode dargestellt werden sollten, wurde innerhalb dieses Gebietes die IntensivUntersuchungsfläche "BongPyeong" mit einer Größe von 11 km * 7 km ausgeschieden. In Abbildung 3.1 ist die Lage des Untersuchungsgebietes graphisch dargestellt.
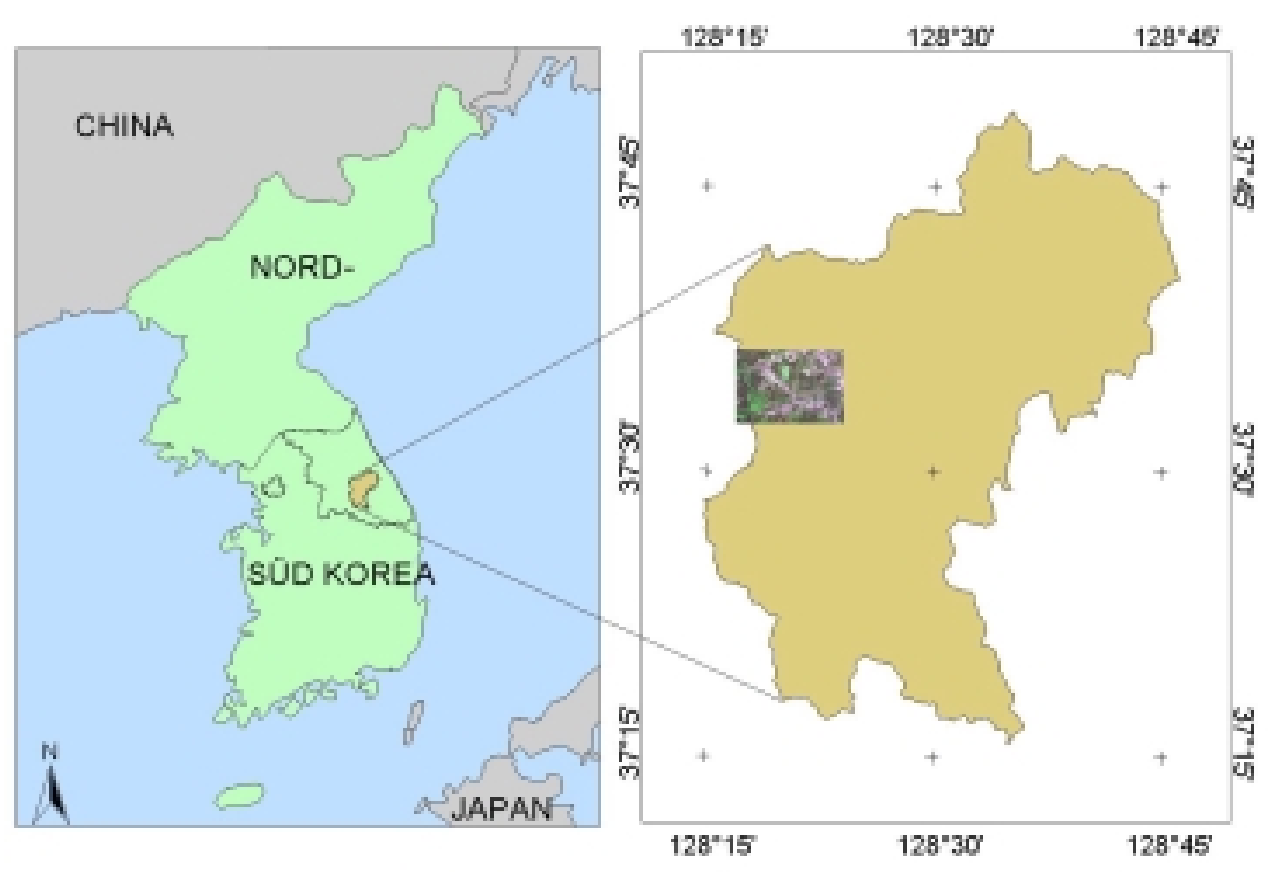

Abbildung 3.1: $\quad$ Lage des Untersuchungsgebietes 


\subsection{Klima}

Korea gehört zur gemäßigten Klimazone und hat vier ausgeprägte Jahreszeiten. Das Klima wird im Winter durch eine kalte Hochdruck-Wetterlage über Sibirien und im Sommer durch eine Hochdrucklage über dem Nordpazifik stark beeinflußt. Die vorherrschenden Windrichtungen sind im Sommer südwestlich und im Winter nordwestlich. Im Vergleich zu europäischen Ländern, die auf dem gleichen Breitengrad wie Südkorea liegen, ist der Sommer in Korea relativ heißer und feuchter mit starken Regenfällen. Der Winter ist kalt und trocken mit gelegentlichen Schneefällen.

Die mittleren Jahrestemperaturen schwanken zwischen $10{ }^{\circ} \mathrm{C}$ und $16{ }^{\circ} \mathrm{C}$. Im wärmsten Monat August erreicht die mittlere Temperatur $25^{\circ} \mathrm{C}$, im kältesten Monat Januar $-0,7$ ${ }^{\circ}$ C. Der jährliche Niederschlag liegt bei ca. $1500 \mathrm{~mm}$ im südlichen Teil von Korea und bei ca. $1300 \mathrm{~mm}$ im zentralen Teil. Mehr als die Hälfte des jährlichen Niederschlages fällt in der Monsunzeit von Juni bis August. Der Winter-Niederschlag beträgt weniger als $10 \%$ von der Gesamtsumme. Die mittlere monatliche Luftfeuchtigkeit erreicht im Juli die höchsten Werte von $70 \%$ bis $80 \%$, im Januar bis April die niedrigsten Werte von $30 \%$ bis $40 \%$. Die Trockenheit im Frühjahr führt häufig zu Zuwachseinbußen bei Waldbäumen und erhöht die Waldbrandgefahr. Da es keine Wetterstation im Untersuchungsgebiet gibt, müssen zur klimatischen Charakterisierung die Informationen der Wetterstation "Wonju", welche ca. $50 \mathrm{~km}$ westlich entfernt liegt, herangezogen werden. In Tabelle 3.1 sind klimatische Kenndaten der letzten 10 Jahre angegeben (KMA, Korea METEOROGiCAl AdMinistration, 2001).

Tabelle 3.1: $\quad$ Mittlere Temperaturen und Niederschläge von 1991 bis 2000

\begin{tabular}{|c|c|c|c|c|c|c|c|c|c|c|c|c|c|c|}
\hline \multicolumn{2}{|l|}{ Monat } & 1 & 2 & 3 & 4 & 5 & 6 & 7 & 8 & 9 & 10 & 11 & 12 & Mittel \\
\hline \multirow{3}{*}{$\begin{array}{c}\text { Temperaturen } \\
\left({ }^{\circ} \mathrm{C}\right)\end{array}$} & Mittel & $-3,5$ & $-1,4$ & 4,8 & 11,9 & 17,0 & 21,7 & 24,8 & 24,7 & 19,4 & 12,4 & 5,3 & $-1,0$ & 11,3 \\
\hline & Min & $-8,5$ & $-7,0$ & $-1,2$ & 4,9 & 10,8 & 16,6 & 20,8 & 20,7 & 14,6 & 6,7 & 0,0 & $-5,9$ & 6,0 \\
\hline & Max & 2,3 & 5,1 & 11,2 & 19,0 & 23,5 & 27,5 & 29,8 & 29,8 & 25,7 & 19,6 & 11,8 & 4,9 & 17,5 \\
\hline \multicolumn{2}{|c|}{$\begin{array}{l}\text { Niederschlag } \\
(\mathrm{mm})\end{array}$} & 16 , & 22 & 43, & 64 & 97 & 14 & 30 & 32 & 14 & 6 & & 27 & $\begin{array}{c}1298,0 \\
\text { (Summe) }\end{array}$ \\
\hline
\end{tabular}




\subsection{Geologie und Boden}

Das geologische Ausgangsmaterial des Untersuchungsgebietes besteht aus einer Mischung von Gneis und Kalksteinen. Aufgrund hoher Temperaturen und hoher Niederschläge im Sommer verwittern die Urgesteine rasch und bilden Böden, die zwar physikalisch günstige Eigenschaften aufweisen, aber durch die Auswaschung nährstoffarm sind (CHUNG, 1996). Es besteht eine Tendenz zur Ausbildung subtropischer Böden (BEON, 1997). Nach einer Gliederung des koreanischen Forstministeriums, welche Waldböden in 8 Gruppen untergliedert, wird der vorherrschende Bodentyp in die Kategorie „Waldbraunerde“ eingeordnet.

\subsection{Untersuchungsmaterial}

\subsection{Luftbilder}

Insgesamt konnten 35 infrarot-schwarz-weiß Luftbilder, die im Jahr 1992 im Auftrag des „Korea Forest Research Institute (KFRI)“ im Maßstab 1:15000 aufgenommen wurden, vom "Institute of Natural Environment Information, Division of Environmental Science \& Ecological Engineering, Korea University" zur Verfügung gestellt werden. Die technischen Daten des Luftbildmaterials sind in Tabelle 3.2 beschrieben.

\section{Tabelle 3.2: $\quad$ Technische Daten des verwendeten Luftbildmaterials}

\begin{aligned} & \hline Aufnahmedatum und Uhrzeit 01. Oktober 1992 / gegen 11 Uhr \\ & Kamera Wild 15/4 UAGA-F \\ & Brennweite $152.39 \mathrm{~mm} \\ &$ Filmtyp Infrarot Schwarz-Weiß \\ & Mittlere Flughöhe ca. 2000 m über Grund \\ & Mittlerer Bildmaßstab $1: 13000 \\ &$ Geplanter Bildmaßstab $1: 15000 \\ &$ Überdeckung(Längs/Quer) $60 / 20 \% \\ &$ Anzahl der Luftbilder 35 \\ & \hline\end{aligned}

\subsection{Multispektrale Satellitendaten}

In den letzten Jahren hat sich gezeigt, daß die Satellitenfernerkundung ein sehr wertvolles Hilfsmittel zur Waldinventur und zum Management natürlicher Ressourcen darstellt. Tabelle 3.3 listet wichtige technische Angaben von optischen Sensoren, die 
bisher für thematische Kartierungen und zum Monitorring der Erdoberfläche am häufigsten eingesetzt wurden sowie diejenigen einiger ausgewählter Sensoren der neuen Generation, die bereits heute bzw. in der nahen Zukunft operationell sein werden auf.

Eine ausführliche Beschreibung über die bereits verfügbaren bzw. sich in der Entwicklung befindlichen Erdbeobachtungssysteme sind beispielsweise bei RICHARDS(2000) oder im Internet Seite http://www.asprs.org/asprs/news/satellites/ zu finden.

Tabelle 3.3: $\quad$ Übersicht der wichtigsten Erdbeobachtungssatelliten.

\begin{tabular}{|c|c|c|c|c|c|c|c|c|c|c|}
\hline \multicolumn{2}{|c|}{ Satellitensystem } & $\begin{array}{c}\text { IRS-1 } \\
\text { C/D } \\
\end{array}$ & SPOT 4 & $\begin{array}{c}\text { Landsat } \\
7 \\
\end{array}$ & Ikonos 2 & $\begin{array}{c}\text { Quick } \\
\text { Bird } \\
\end{array}$ & $\begin{array}{c}\text { OrbView } \\
3 / 4 \\
\end{array}$ & EROS B & ALOS & $\begin{array}{c}\text { SPOT } \\
5 \mathrm{~A} \\
\end{array}$ \\
\hline \multicolumn{2}{|c|}{ Status } & \multicolumn{5}{|c|}{ In Betrieb } & \multicolumn{4}{|c|}{ In Entwicklung } \\
\hline \multicolumn{2}{|c|}{ Eigentümer } & ISRO & $\begin{array}{l}\text { SPOT } \\
\text { Image }\end{array}$ & $\begin{array}{l}\text { NASA } \\
\text { NOAA }\end{array}$ & $\begin{array}{c}\text { Space } \\
\text { Imaging }\end{array}$ & $\begin{array}{l}\text { Digital } \\
\text { Glove }\end{array}$ & $\begin{array}{l}\text { Orb- } \\
\text { image }\end{array}$ & ImageSat & NASDA & $\begin{array}{l}\text { SPOT } \\
\text { Image }\end{array}$ \\
\hline \multicolumn{2}{|c|}{ Start } & $\begin{array}{c}1995 / \\
1997\end{array}$ & 1998 & 1999 & 1999 & 2001 & - & 2003 & 2003 & 2002 \\
\hline \multirow{7}{*}{$\begin{array}{c}\text { Band } \\
\text { Spektral } \\
(\mu \mathrm{m})\end{array}$} & Blau & & & $\begin{array}{l}0.45- \\
0.52\end{array}$ & $\begin{array}{c}0.45- \\
0.52\end{array}$ & $\begin{array}{c}0.45- \\
0.52\end{array}$ & $\begin{array}{c}0.45- \\
0.52\end{array}$ & & $\begin{array}{c}0.42- \\
0.50\end{array}$ & \\
\hline & Grün & $\begin{array}{c}0.52- \\
0.59\end{array}$ & $\begin{array}{c}0.50- \\
0.59\end{array}$ & $\begin{array}{c}0.53- \\
0.61\end{array}$ & $\begin{array}{c}0.52- \\
0.60\end{array}$ & $\begin{array}{c}0.52- \\
0.60\end{array}$ & $\begin{array}{c}0.52- \\
0.60\end{array}$ & 4 Band & $\begin{array}{c}0.52- \\
0.60\end{array}$ & $\begin{array}{c}0.50- \\
0.59\end{array}$ \\
\hline & Rot & $\begin{array}{c}0.62- \\
0.68\end{array}$ & $\begin{array}{c}0.61- \\
0.68\end{array}$ & $\begin{array}{c}0.63- \\
0.69\end{array}$ & $\begin{array}{c}0.63- \\
0.69\end{array}$ & $\begin{array}{c}0.63- \\
0.69\end{array}$ & $\begin{array}{c}0.625- \\
0.695\end{array}$ & & $\begin{array}{c}0.61- \\
0.69\end{array}$ & $\begin{array}{c}0.61- \\
0.68\end{array}$ \\
\hline & NIR & $\begin{array}{c}0.77- \\
0.86\end{array}$ & $\begin{array}{c}0.79- \\
0.89\end{array}$ & $\begin{array}{c}0.75- \\
0.90\end{array}$ & $\begin{array}{c}0.76- \\
0.90\end{array}$ & $\begin{array}{c}0.76- \\
0.90\end{array}$ & $\begin{array}{c}0.76- \\
0.90\end{array}$ & & $\begin{array}{c}0.76- \\
0.89\end{array}$ & $\begin{array}{c}0.79- \\
0.89\end{array}$ \\
\hline & $\begin{array}{l}\text { MIR } \\
\text { MIR }\end{array}$ & $\begin{array}{l}1.55- \\
1.75\end{array}$ & $\begin{array}{l}1.58- \\
1.75\end{array}$ & $\begin{array}{c}1.55- \\
1.75 \\
2.09- \\
2.35\end{array}$ & & & & & & $\begin{array}{l}1.58- \\
1.75\end{array}$ \\
\hline & THIR & & & $10.4-2.5$ & & & & & & \\
\hline & Pan. & $\begin{array}{l}0.5- \\
0.75\end{array}$ & $\begin{array}{c}0.61- \\
0.68\end{array}$ & $\begin{array}{c}0.52- \\
0.90 \\
\end{array}$ & $\begin{array}{c}0.45- \\
0.90\end{array}$ & $\begin{array}{c}0.445- \\
0.90\end{array}$ & $\begin{array}{c}0.45- \\
0.90 \\
\end{array}$ & $\begin{array}{c}0.50- \\
0.90 \\
\end{array}$ & $\begin{array}{c}0.52- \\
0.77\end{array}$ & $\begin{array}{c}0.51- \\
0.73 \\
\end{array}$ \\
\hline \multirow{3}{*}{ Auflösung } & $\mathrm{G}(\mathrm{m})$ & $\begin{array}{l}5.6 / \\
23.5\end{array}$ & $10 / 20$ & $15 / 30$ & $1 / 4$ & $\begin{array}{c}0.61 / \\
2.44\end{array}$ & $1 / 4$ & 0.82 & $2.5 / 10$ & $\begin{array}{c}2.5-5 / \\
10\end{array}$ \\
\hline & $\mathrm{R}$ (bit) & $6 / 7$ & 8 & 8 & 11 & 11 & 11 & $8 / 11$ & KA & 8 \\
\hline & $\begin{array}{c}\mathrm{T} \\
\text { (Tage) }\end{array}$ & 5 & 4 & 16 & $<3$ & $<3.5$ & $<3$ & 1 & 2 & $2-3$ \\
\hline \multicolumn{2}{|c|}{ Stereoaufnahme } & $\mathrm{Ja}$ & $\mathrm{Ja}$ & Nein & $\mathrm{Ja}$ & $\mathrm{Ja}$ & $\mathrm{Ja}$ & KA & Ja & $\mathrm{Ja}$ \\
\hline \multicolumn{2}{|c|}{ Altitude $(\mathrm{km})$} & 817 & 822 & 705 & 681 & 450 & 470 & $480 / 600$ & 692 & 830 \\
\hline \multicolumn{2}{|c|}{ Streifenbreite $(\mathrm{km})$} & $70 / 142$ & 60 & 185 & 11 & 16.5 & 8 & $12.5 / 16$ & 70 & 60 \\
\hline
\end{tabular}

Im folgenden soll ein kurzer Überblick über die satellitengestützten Fernerkundungssysteme, die in der vorliegenden Arbeit Verwendung fanden, gegeben werden. 


\subsection{Landsat}

Mit dem Start des ERTS-A (Earth Resources Technology Satellite) Programms am 23. Juli 1972 begann die Ära der zivilen Erderkundungssatelliten. Kurz vor dem Start des ERST-B am 22. Januar 1975 wurde das Programm in Landsat umgenannt. Landsat 1-3 hatten neben einer "Return Beam Vidicon (RBV)" Kamera einen multispektralen Sensor (MSS) an Bord. Landsat 4 und der baugleiche Landsat 5 brachten als eine wesentliche Weiterentwicklung des Landsat-Programms neben dem Einsatz des MSS einen neuartigen Thematic Mapper (TM) hervor.

Im Vergleich zu MSS Daten verfügt Landsat TM über eine erhöhte radiometrische Auflösung mit 8bit, eine verbesserte geometrische Auflösung mit 30m x 30m und eine erweiterte spektrale Auflösung mit 7 Bänden im visuellen, nahinfraroten und thermalen Bereich. Landsat 5 befindet sich seit seinem Start am 1. März 1984 ununterbrochen im Einsatz. Wegen der hohen Betriebskosten ist jedoch vor kurzem das Ende des Betriebes angekündigt worden (USGS Pressemitteilung, 2001). Landsat 6 scheiterte durch einen technischen Defekt. Am 15. April 1999 wurde Landsat 7 mit einem "Enhanced Thematic Mapper Plus (ETM+)" in den Weltraum gebracht. Der ETM+ besitzt ein zusätzliches panchromatisches Band mit $15 \mathrm{~m}$ geometrischer Auflösung und ist damit ein acht-band-multispektraler Sensor. Ausführliche technische Daten des Landsat ETM+ und aktuelle Informationen über das Landsat-Programm sind im Internet unter der Internet Adresse http://landsat7.usgs.gov/index.html zu finden

Für die vorliegenden Untersuchungen wurde eine Landsat 5 TM Szene vom 02. November 1992 vom "Institute of Natural Environment Information, Division of Environmental Science \& Ecological Engineering, Korea University" zur Verfügung gestellt. In Tabelle 3.4 sind die technischen Daten der verwendeten Landsat TM Szene angegeben.

Tabelle 3.4: $\quad$ Technische Daten der verwendeten Landsat 5 TM Szene

\begin{aligned} & \hline Sensor Landsat 5 TM \\ & Aufnahmedatum / Uhrzeit 02. Nov. 1992 / 01:26 \\ & Path/ Row 115 / 34 \\ & Sonne Elevation / Azimuth 32.12 / 149.66 Degrees \\ & Karten Projektion / Datum UTM, Zone 52 / WGS84 \\ & Resampling Method Cubic Convolution \\ & \hline\end{aligned}




\subsection{IKONOS}

Nach einem vorhergehenden Mißerfolg ist es der Firma Space Imaging (SI) gelungen, am 24 September 1999 mit einer Lockheed Martin Athena II Rakete den IKONOS-2 Satelliten in den Weltraum $\mathrm{zu}$ bringen. IKONOS-2 ist der erste kommerzielle Fernerkundungssatellit mit einer sehr hohen geometrischen Auflösung und stellt somit den Beginn eines neuen Zeitalters der Satelliten-Fernerkundung dar. Der Satellit fliegt in ca. 680 Kilometer Höhe in einer sonnensynchronen Umlaufbahn mit 98 Minuten für einen Erdumlauf. Der Sensor ist „off-nadir“ Aufnahmen bis zu 60 Grad fähig. Damit kann der IKONOS-2 innerhalb 3 Tage das selbe Gebiet erneut aufnehmen und weiterhin sind damit Stereo-Aufnahme möglich. IKONOS-2 verfügt sowohl über einen panchromatischen als auch über multispektrale Sensoren. Die höchst mögliche geometrische Auflösung beträgt ca. $0.85 \mathrm{~m}$ im Nadir. Daraus werden unter dem Produktnamen CARTERA ${ }^{\mathrm{TM}}$ verschiedene Produkte abgeleitet. Diese Produkte werden mit einer räumlichen Auflösung von $1 \mathrm{~m}$ x 1m für die panchromatischen und $4 \mathrm{~m}$ x $4 \mathrm{~m}$ für die multispektralen Daten in unterschiedlicher geometrischen Genauigkeit und Preis angeboten.

Die für die vorliegende Arbeit angeschaffte, wolkenfreie IKONOS-Szene wurde am 24. April 2000 aufgenommen und als GEO-Produkt bezogen. In der Tabelle 3.5 sind wichtige technische Daten der verwendeten IKONOS Szene beschrieben. Vollständigen „Image Metadaten“ sind im Anhang 1 angegeben. Die detaillierten geometrischen Charakteristiken der IKONOS-GEO Daten werden in Abschnitt 5.322 erläutert.

\section{Tabelle 3.5: Technische Daten der verwendeten IKONOS Szene}

\begin{aligned} & \hline Sensor IKONOS-2 \\ & Aufnahmedatum / Uhrzeit 24. Apr. 2000 / 01:46 \\ & Sonne Elevation / Azimuth 57.10895 / 131.5839 Degrees \\ & Sensor Elevation / Azimuth 57.51640 / 140.1404 Degrees \\ & Vorprozeß Level Standard Geometrically Corrected \\ & Karten Projektion / Datum UTM, Zone 52 / WGS84 \\ & Resampling Methode Bicubic \\ & File Format GeoTIFF, 11 bit per Pixel \\ & \hline\end{aligned}




\subsection{Weitere Geometrie- und Sachdaten}

\subsection{Topographische Karten und digitales Geländemodell}

Die topographischen Karten im Maßstab 1:25000 vom "National Geography Institute (NGI)" Koreas wurden zur Gewinnung der Paßpunkte und Generierung eines digitalen Geländemodells verwendet. Diese Karten lagen sowohl in analoger als auch in digitaler Form vor. Sie enthalten Angaben über das Wegenetz, Flüsse, die Landnutzungsform, Ortschaftsgrenzen und die Lage von Gebäuden. Weiterhin sind auf den Karten Höhenangaben in Form von Konturlinien im Abstand von 10m enthalten. Da die Luftbilder nicht flächendeckend vorlagen, wurden die Höhenlinien aus den digitaltopographischen Karten extrahiert und mit Hilfe von ArcInfo ein digitales Geländemodel berechnet.

\subsection{Waldtypenkarten}

Außer den topographischen Karten wurden Waldtypenkarten des KFRI als Hilfsmittel für die Auswertung der Luftbilder und Satellitendaten einbezogen. Es war geplant, dieses Material als Referenzdaten zu verwenden. Nach einer Voruntersuchung wurde jedoch festgestellt, daß die Karten als Referenzdaten nicht geeignet sind. Obwohl die Waldtypenkarten von einer Auswertung der selben Luftbilder stammen, welche in den vorliegenden Untersuchungen verwendet wurden, waren die Fehler der geometrischen und thematischen Angaben relativ groß. Dies ist vermutlich auf wenig geeignete Auswertungsgeräte bei der Erstellung der Waldtypenkarten zurückzuführen.

\subsection{Terrestrische Aufnahmedaten}

Durch eine Feldaufnahme wurden die Referenzdaten für die Auswertung des IKONOS Satellitenbildes gewonnen. Zur Identifizierung der einzelnen Landnutzungstypen und Baumarten war eine terrestrische Aufnahme nicht zu vermeiden, obwohl die IKONOS Daten ein sehr gutes geometrisches und radiometrisches Auflösungsvermögen haben. 


\section{Methode}

\subsection{Digitale Luftbildauswertung}

Unter digitaler Photogrammetrie, auch „Softcopy“ Photogrammetrie genannt, wird die Auswertung digitaler Luftbilder verstanden. Die Arbeitschritte der „Softcopy“ Photogrammetrie weichen im wesentlichen nicht von den als „Hardcopy“ Photogrammetrie bezeichneten analogen oder analytischen Verfahren ab. Jedoch hat die digitale Photogrammetrie viele Vorteile gegenüber der „Hardcopy“ Photogrammetrie. PRÖBSTING (1996) faßt die Vor- und Nachteile der digitalen Photogrammetrie gegenüber der analytischen Photogrammetrie zusammen. Als wesentliche Unterschiede sind u.a. folgende zu erwähnen:

Vorteile: - Hohe Transportabilität

- Keine optisch- mechanische Instrumente erforderlich

- Halb- bzw. vollautomatische Innere und Relative Orientierung

- Automatische und halbautomatische Bildauswertung

- Integration mit Orthophotoherstellung und GIS

- Beliebige Vervielfältigung der Bilddaten ohne Qualitätsverlust

- Bildverbesserungsmöglichkeiten

Nachteile: - Lange Rechenzeiten

- Hoher Speicherplatzbedarf

- Verminderte Qualität der Stereobetrachtung

- Verminderte Bildqualität gegenüber den Originalluftbildern

Die Vorteile der digitalen Photogrammetrie bestehen nicht nur in den oben genannten Punkten, sondern auch in der Tatsache, daß die innere Orientierung nur einmal durchgeführt werden muß und die Bilder dann sehr schnell in orientiertem Zustand für das System immer wieder verfügbar sind.

BaltSAVIAS (1999) beschreibt den Hauptanwendungsbereich der Softcopy Photogrammetrie als

- die digitale Orthophoto Herstellung,

- die automatische Aerotriangulation,

- die automatische DTM-Generation, 
- Aufbau und Update von digitalen Merkmalsdatenbanken und

- die Integration von digitalen Daten, insbesondere DTMs, Orthophotos und sich daraus ableitenden Produkte in ein GIS.

Bei der vorliegenden Untersuchung wurden die Luftbilder sowohl für die Bereitstellung der Referenzdaten (Ground Truth) für die Klassifikation der Landsat TM Daten aus dem Jahr 1992 und zur Überprüfung der Klassifikationsgenauigkeit als auch für die Gewinnung von Paßpunkten für die geometrische Korrektur der IKONOS Daten herangezogen. Die Auswertungen erfolgten an der institutseigenen digitalen photogrammetrischen Arbeitstation Softplotter Ver. 1.8.3 von der Firma Vision International. In diesem Kapital wird nicht in die Einzelheiten der photogrammetrischen Methoden eingegangen, sondern es werden einige Besonderheiten der digitalen Photogrammetrie gegenüber der traditionellen anlogen oder analytischen Photogrammetrie beschrieben. Ausführliche Informationen über Photogrammetrie sind u.a. in Huss et. al. (1984), HILDEBRANDT (1996) und KRAUS (1996) zu finden.

\subsection{Scannen des Bildmaterials}

Digitale Bilder können direkt in digitaler Form mit Hilfe einer digitalen Kamera oder eines elektronisch-optischen Scanners aufgenommen werden. Für die Auswertung von analogen Luftbildern mit Hilfe der „Softcopy“ Photogrammetrie muß zunächst das Bildmaterial durch Scannen digitalisiert werden. Die Qualität der gescannten Bilder hängt zum einen vom Bildmaterial selber, zum anderen von dem verwendeten Scanner ab. Der Scanner soll geometrisch korrekt und radiometrisch präzise Ergebnisse liefern.

Vor dem Scannen stellt sich die Frage nach der räumlichen Auflösung. Eine allgemein gültige optimale Auflösung zu nennen ist schwer, weil die optimale geometrische Auflösung von dem Anwendungszweck und den Benutzeranforderungen abhängig ist. Um Informationsverlust beim Scannen $\mathrm{zu}$ vermeiden ist die ideale Pixelgröße offensichtlich die Größe, die der ursprünglichen Auflösung des analogen Filmes entspricht. Für hochauflösende Filme und Luftbildkameras kann eine Auflösung von 60 lp/mm angenommen werden (MEIER 1984). Dies würde einer Pixelgröße von ungefähr $6 \mu \mathrm{m}$ entsprechen (BALTSAVIAS UND BILL, 1994) und resultiert für ein $23 \mathrm{~cm} * 23 \mathrm{~cm}$ großes Luftbild in einer ca. 1.4 Gigabyte großen Datei. Bei Farbbildern wird das Dreifache erreicht. Trotz einer schnellen Entwicklung zu immer leistungsfähigen 
Rechnern wird es Schwierigkeiten bei der Behandlung dieser Datenmengen geben. Außerdem hängt die Bildqualität nicht nur von der Pixelgröße sondern auch von den Merkmalen des gesamten Scannersystems einschließlich Beleuchtung, Optik, Filtern, Sensor, Analog-Digital Konverter, „Hardware“ und „Software“bearbeitung etc. ab (BAltsavias und BiLl, 1994). Deswegen müssen in der Praxis gewisse Kompromisse von den Benutzern eingegangen werden.

Für eine DTM Erstellung gibt es zahlreiche Untersuchungen, die mit einer Pixelgröße von 10-30 $\mu \mathrm{m}$ ein zufriedenstellendes Ergebnis erzielt haben. KRZYSTEK und WILD (1992) berichteten über Genauigkeitsvergleiche für eine DTM Erstellung mit Hilfe von zwischen 15 und $30 \mu \mathrm{m}$ gescannten Bildern. Der Genauigkeitsunterschied zwischen den zwei Versionen war gering und die $30 \mu \mathrm{m}$-Version hatte eine Genauigkeit von ca. 0.01 $\%$ der Flughöhe über dem Gelände. In vielen Orthophoto Projekten wurden Luftbilder mit einer Pixelgröße von $25 \mu \mathrm{m}$ oder mehr gescannt. Für eine hohe geometrische Genauigkeit kann ein Scannen mit 600-1200 dpi (42 - $21 \mu \mathrm{m})$ hinreichend sein, wobei 1200 dpi die Erkennung der meisten Merkmale erlaubt. Für eine visuelle Interpretation besonders in städtischen und aufgeforsteten Gebieten ist jedoch eine noch höhere Auflösung wünschenswert (SKALET et. al., 1992).

Vom praktischen Gesichtpunkt her scheint die Grenze für eine operationale Handhabung und interaktive Arbeit bei 15 - $20 \mu \mathrm{m}$ zu liegen (BALTSAVIAS und BILL, 1994). In Tabelle 4.1 sind Beispiele für die Datenmenge je Scannauflösung und dementsprechender Bodenauflösung getrennt für verschiedene Bildmaßstäbe angegeben.

Wegen einer schnellen Entwicklung der Technik und günstigen Preisverhältnissen ist die Nutzung von DTP (DeskTop Publishing) Scannern für photogrammetrische Aufgaben möglich. BALTSAVIAS et. al. (1995, 1996) haben die Qualität der DTP Scanner überprüft und festgestellt, daß eines der Hauptprobleme von DTP Scannern für photogrammetrische Auswertungen die geringe geometrische Genauigkeit ist. Trotz der geringen geometrischen Genauigkeit können DTP Scanner für gewisse photogrammetrische Anwendungen benutzt werden. Für eine Herstellung analoger Orthophotos oder Ortophotokarten kann die geometrische Genauigkeit solcher Scanner genügen, während sie für genaue photogrammetrische Auswertungen im allgemeinen nicht ausreicht. 
Tabelle 4.1: Beispiele für die Dateigröße je Scannauflösung und dementsprechender Bodenauflösung für verschiedene Bildmaßstäbe.

\begin{tabular}{c|cccccccc}
\hline & \multicolumn{7}{|c}{ Auflösung beim Scannern } \\
\hline Mikron & 10 & 12 & 20 & 24 & 40 & 48 & 60 & 96 \\
dpi & 2540 & 2116 & 1270 & 1058 & 635 & 529 & 423 & 265 \\
Dateigröße $(\mathrm{Mb})^{*}$ & 504.5 & 350.3 & 126.1 & 87.6 & 31.5 & 21.9 & 14.0 & 5.8 \\
\hline
\end{tabular}

*Beim Farbscannen werden die Daten 3-fach größer als die angegebenen Größen

\begin{tabular}{c|cccccccc}
\hline Bild Maßstab & \multicolumn{7}{|c}{ Bodenauflösung $(\mathrm{mm})$} \\
\hline $1: 1000$ & 10 & 12 & 20 & 24 & 40 & 48 & 60 & 96 \\
$1: 2000$ & 20 & 24 & 40 & 48 & 80 & 96 & 120 & 192 \\
$1: 3000$ & 30 & 36 & 60 & 72 & 120 & 144 & 180 & 288 \\
$1: 4000$ & 40 & 48 & 80 & 96 & 160 & 192 & 240 & 384 \\
$1: 5000$ & 50 & 60 & 100 & 120 & 200 & 240 & 300 & 480 \\
$1: 6000$ & 60 & 72 & 120 & 144 & 240 & 288 & 360 & 576 \\
$1: 7000$ & 70 & 84 & 140 & 168 & 280 & 336 & 420 & 672 \\
$1: 8000$ & 80 & 96 & 160 & 192 & 320 & 384 & 480 & 768 \\
$1: 9000$ & 90 & 108 & 180 & 216 & 360 & 432 & 540 & 864 \\
$1: 10000$ & 100 & 120 & 200 & 240 & 400 & 480 & 600 & 960 \\
$1: 20000$ & 200 & 240 & 400 & 480 & 800 & 960 & 1200 & 1920 \\
$1: 30000$ & 300 & 360 & 600 & 720 & 1200 & 1440 & 1800 & 2880 \\
$1: 40000$ & 400 & 480 & 800 & 960 & 1600 & 1920 & 2400 & 3840 \\
\hline
\end{tabular}

\subsection{Aerotriangulation}

Für die Orientierung von mehreren Bildflugstreifen wird die sogenannte Aerotriangulation zur photogrammetrischen Punktbestimmung herangezogen. Aerotriangulation ist eine photogrammetrische Methode zur Ermittlung von Paßpunktkoordinaten aus Luftbildstreifen oder auch Luftbildblöcken. In „Softcopy“ Photogrammetrie werden dabei für ein Bildstreifen oder auch -block durch Messung der Koordinaten von Paß- und Verknüpfungspunkten in den Bildern gleichzeitig die Orientierungsparameter aller Bilder bestimmt. Der wesentliche Vorteil dieser Methode liegt darin, daß im Vergleich zur Einzelbildorientierung deutlich weniger Paßpunkte benötigt werden und die Genauigkeit nur unwesentlich gegenüber der Orientierung von Einzelbildern abnimmt. Voraussetzung für das Verfahren ist eine Luftbildaufnahme des Blocks mit mindestens $60 \%$ Längsüberdeckung und $20 \%$ Querüberdeckung.

Die Aerotriangulation wird im allgemein nach zwei Methoden durchgeführt (KRAUS, 1996): Blockausgleichung mit unabhängigen Modellen und Bündelblockausgleichung 
Die Blockausgleichung mit unabhängigen Modellen beginnt mit Modellkoordinaten, die durch rechnerische relative Orientierung und Modellbildung aus dem jeweiligen Überlappungsbereich von zwei Meßbildern gewonnen werden. Im Rahmen der Blockausgleichung werden die einzelnen Modelle $\mathrm{zu}$ einem Modellverband verschmolzen und in das übergeordnete Landeskoordinatensystem überführt. Bei einer Aerotriangulation mit unabhängigen Modellen ist das einzelne Stereomodell die Elementareinheit.

Die Bündelblockausgleichung ist die strengste Methode der photogrammetrischen Punktbestimmung. Ausgangsmaterial ist ein streifen- oder blockförmiger Bildverband. Bei der Bündelblockausgleichung wird auf rechnerische Weise der Zusammenhang zwischen den Bildkoordinaten und den Objektkoordinaten direkt, d.h. ohne Umweg über Modellkoordinaten, hergestellt. Bei der Bündelblockausgleichung ist also das Bild die Elementareinheit. Abbildung 4.1 zeigt dieses Grundprinzip.

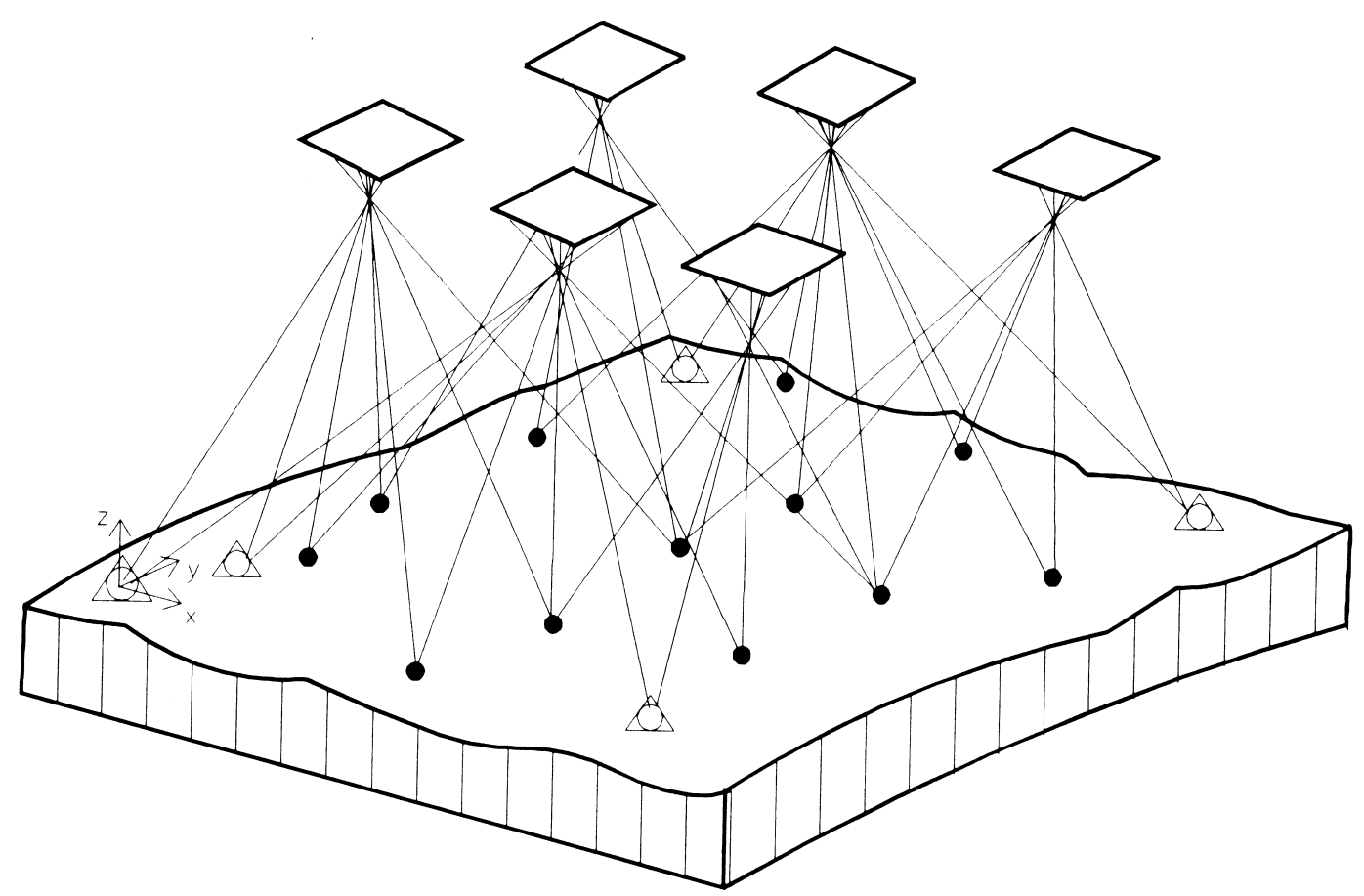

Abbildung 4.1:Grundprinzip der Bündelblockausgleichung (KRAUS 1996)

Die Bildkoordinaten und das dazugehörige Projektionszentrum definieren ein räumliches Strahlenbündel. Die äußeren Orientierungselemente der Strahlenbündel eines Blockverbandes werden für alle Bilder gleichzeitig bestimmt. Als Information stehen dafür die Bildkoordinaten der Verknüpfungspunkte (Punkte, die in mehr als 
einem Bild vorkommen) und die terrestrischen Koordinaten der Paßpunkte zur Verfügung.

\subsection{Stereo Auswertung}

Die stereoskopische Auswertung von Luftbildern ermöglicht durch die Einbeziehung der dritten Dimension eine bessere Erkennung der Objekte. Bei der stereoskopischen Luftbildinterpretation wird z.B. die Höheninformation zu den Merkmalen wie Textur, Form und Große, Lage und Schatten mit herangezogen. Eine stereoskopische Betrachtung ist wesentlich angenehmer und sicherer als eine monoskopische Betrachtung. Bei einer Untersuchung für die Erfassung der Veränderung konnte gezeigt werden, daß durch eine räumliche Betrachtung etwa 50\% mehr Veränderungen erfaßt und wesentlich weniger Fehlinterpretation gemacht wurden (KRAUS, 1996).

Die räumliche Betrachtung bei dem in die Untersuchung herangezogenen digitalen photogrammetrischen System „Softplotter“ basiert auf der Bildblenden-Technik. Bei dieser Methode wird jedes Einzelbild eines Bildpaares mit $120 \mathrm{hz}$ Frequenz aufeinanderfolgend eingeblendet. Dabei sorgt eine spezielle Brille „Crystal Eye“ dafür, daß die Bildblenden im Monitor mit den Augen synchronisiert werden. So sieht man das linke Bild mit dem linken Auge, wenn im Monitor das linke Bild eingeblendet wird und entsprechend das rechte Bild mit dem rechten Auge.

\subsection{Digitale Orthophotoherstellung}

Ein Orthophoto ist ein differentiell entzerrtes und dadurch von der Zentralperspektive in eine Orthogonalprojektion gebrachtes Luftbild. Unabhängig von der Geländegestalt werden im Orthophoto die Fülle an Informationen des Luftbildes mit der Geometrie einer topographischen Karte verbunden.

Die digitale Orthophoto-Technologie bietet bedeutende Vorteile gegenüber traditionellen analogen Verfahren. Dies sind u.a. die verbesserte Bildqualität, die Integration mit GIS sowie eine rechnergestützte Bildinterpretation. Digital hergestellte Orthophotos sind weiterhin billiger als analog erzeugte (BÄHR und WIESEL, 1991).

Für die Herstellung digitaler Orthophotos benötigt man digitale Vorlagebilder und ein digitales Oberflächenmodell. Mit Hilfe des digitalen Oberflächenmodells werden die Bildkoordinaten $(\mathrm{x}, \mathrm{y})$ jedes Pixels im Vorlagebild in Landeskoordinaten $(\mathrm{x}, \mathrm{y}, \mathrm{z})$ 
transformiert. So wird für jedes Bildpaar ein Orthophoto getrennt erzeugt und anschließend werden die einzelnen Orthophotos $\mathrm{zu}$ einem Orthophotomosaik zusammengesetzt Die Abbildung 4.2 zeigt den Zusammenhang zwischen digitalem Oberflächenmodell und Vorlagenbild.

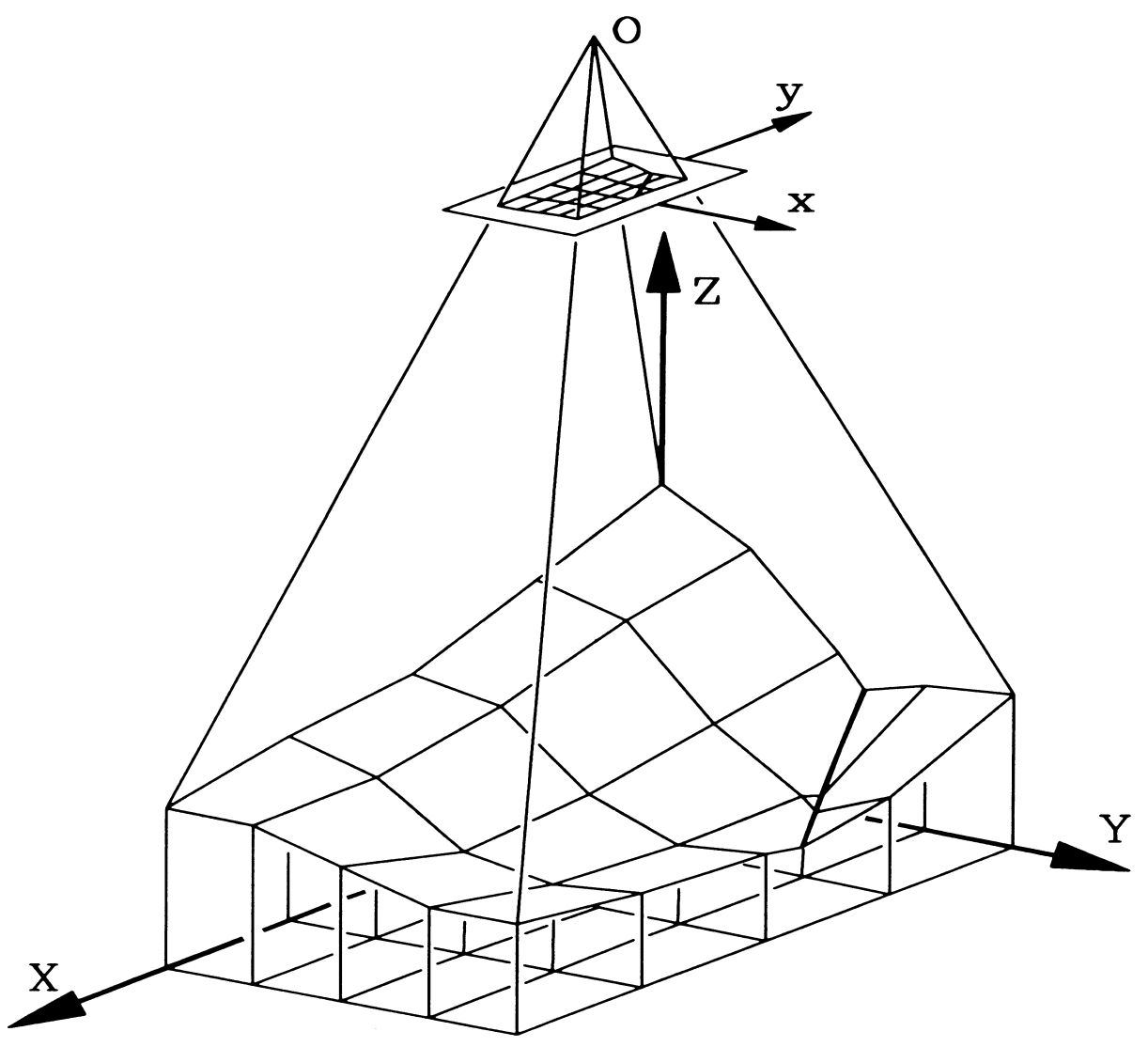

Abbildung 4.2:Zusammenhang zwischen digitalem Oberflächenmodell und Vorlagenbild (KRAUS 1996) 


\subsection{Vorbereitende Arbeit der Satellitenbildauswertung}

\subsection{Geometrische Korrektur}

Die Geometrie einer Satellitenaufzeichnung wird einerseits durch das Aufnahmesystem und andererseits durch Reliefbedingungen des Geländes bestimmt, welche unerwünschte Verzerrungen erzeugen. Die systembedingten Verzerrungen, die auf die Aufnahmeprinzip des Sensors, sowie auf die Bewegungen und Lage des Sensorträgers zurückzuführen sind, werden in der Regel von dem Satellitenbetreiber korrigiert. Die Verzerrungen durch Höhenunterschiede im Gelände und die Abbildungsunterschiede durch unterschiedliche Kartenprojektionen müssen im allgemein von den Benutzern korrigiert werden. Unter Georeferenzierung versteht man die Überführung der Bildkoordinaten in die vorgegebene Kartenprojektion. Diesen Vorgang nennt man auch Geokodierung und ist in der Regel notwendig, wenn multitemporale Daten zur Anwendung kommen und das Bildmaterial mit anderen raumbezogenen Daten in Verbindung gebracht werden soll, oder wenn aus den Satellitendaten selbst Bildkarten erstellt werden sollen.

Grundsätzlich unterscheidet man zwei verschiedene Verfahren der Georefernzierung: parametrische und nichtparametrische Verfahren (HILDEBRANDT 1996, KELLENBERGER 1996). Mit dem parametrischen Ansatz wird versucht, die Aufnahmesituation anhand der Aufnahmeparameter, Sensorposition, -orientierung und Geländehöhe zu rekonstruieren. Nicht parametrische Ansätze als Näherungslösung dienen zur Erfassung der geometrischen Verhältnisse, ohne die Aufnahmesituation zu rekonstruieren. Die Näherungslösungen sind im Vergleich zu den parametrischen Ansätzen wesentlich einfacher und können vom Sensor unabhängig eingesetzt werden. Nachfolgend werden einige Methoden der nichtparametrischen Ansätze erläutert.

\subsection{Polynomtransformation}

In der Praxis kommt als nichtparametrischer Ansatz häufig eine Polynomtransformation mit Hilfe von Paßpunkten (Ground Control Point: GCP) zur Anwendung. Die Gleichungen der Transformation in einem polynom t-ten Grades sind : 


$$
\begin{aligned}
& x_{0}=\left(\sum_{i=0}^{t}\right)\left(\sum_{j=0}^{i}\right) a_{k} x^{i-j} y^{j} \\
& y_{0}=\left(\sum_{i=0}^{t}\right)\left(\sum_{j=0}^{i}\right) b_{k} x^{i-j} y^{j}
\end{aligned}
$$

wobei $t$ die Grade des Polynoms, und $a_{k}$ und $b_{k}$ die Koeffizienten sind.

Die Ausgleichung wird nach der „Methode der kleinsten Quadrate“ durchgeführt. Die Mindestzahl der benötigen GCPs ist abhängig von dem verwendeten Polynomgrad und kann mit der folgenden Formel berechnet werden.

$$
N=\left(\frac{(t+1)(t+2)}{2}\right)
$$

Die Methode der Polynomtransformation ist eine Transformation, in der Verzerrungen durch Geländehöhenunterschiede unberücksichtigt bleiben. Ob die reliefbedingten geometrischen Verzerrungen toleriert werden können, hängt vom Auswertungszweck und von den in der Szene vorkommenden Höhenunterschieden ab. Die reliefbedingten Verzerrungen wirken sich zweifach auf die Genauigkeit der Entzerrung aus: einmal verfälschen sie die der Transformation zugrunde liegenden Bildkoordinaten der Paßpunkte und verursachen dadurch eine fehlerhafte Berechnung der Transformationsgleichungen. Zum anderen sind dadurch die Position der Pixel im Ausgabebild nach der Transformation entsprechend den jeweiligen Geländehöhen mit Lagefehlern behaftet (HILDEBRANDT 1996).

\subsection{Orthorektifizierung}

Mit Erhöhung der geometrischen Auflösung moderner Satellitendaten wurden die Erwartungen für die geometrische Genauigkeit dementsprechend größer. Bei den sog. „off-nadir“ fähigen hochauflösenden Satelliten wie SPOT und IKONOS werden die reliefbedingten Verzerrungen noch größer, wenn eine Szene im „off-nadir“ Modus, der bei der solchen Satellitendaten im allgemeinen der Normalfall ist, aufgenommen wird.

Toutin aus dem „Canada Centre for Remote Sensing (CCRS)“ entwickelte im Jahr 1983 ein nichtparametrisches Verfahren zur Orthorektifizierung für SPOT-HRV Daten. 
Dieses Verfahren wurde für die anderen multispektralen Satellitendaten (Landsat TM, Spot XS/P, IRS, ASTER, KOMPSAT) und SAR Daten (ERS, JERS, SIR-C, und RADARSAT) weiter entwickelt und erfolgreich angewendet (TOUTIN und CHENG, 2000). Das Korrekturmodel basiert auf dem Prinzip der Orbitographie, der Photogrammetrie, der Geodäsie, und der Kartographie. Die Abbildungsgeometrie des Sensors wird dabei mit Informationen über die Position und Geschwindigkeit der Plattform, mit Sensorparametern und mit geometrischen Charakteristiken der Erde und der Kartenprojektion verknüpft (TOUTIN 1995). Eine ausführliche Beschreibung der mathematischen Herleitung des Korrekturmodels ist in den Veröffentlichungen von Toutin (1983), Toutin et al. (1992), und Toutin und CARBOnNEAU (1992) zu finden.

In den letzten Jahren wurde das Model für IKONOS Geo-Daten weiter entwickelt (Toutin und ChEng, 2000). Obwohl die detaillierten Informationen des IKONOS Satelliten noch nicht veroffentlicht wurden, kann sie mit Hilfe der Grundinformationen aus den Metadaten ein Näherungswert für das Korrekturmodel hergeleitet werden. Der Aufnahmewinkel wurde dabei z.B. aus der „Nominal Collection Elevation“ (Winkel zwischen der Erdebene und des Sensors) und der „Nominal Ground Resolution“ (Aufnahme-Auflösung) in beiden Scannrichtungen berechnet.

Dieses Model ist in das kommerzielle Bildverarbeitungssystem PCI OrthoEngine integriert. In der vorliegende Untersuchung wurde die PCI OrthoEngine V8.0 für die Orthorektifizierung der IKONOS Daten verwendet.

\subsection{Topographische Normalisierung}

Die Intensität der Grauwerte in einem Satellitenbild wird von dem Sensor, den atmosphärischen Bedingungen, den georadiometrischen Faktoren (vor allem Topographie, Illumination und Blickwinkel) und der Reflektionscharakteristik der Objekte beeinflußt (TEILLET 1986). Die Variabilität der Grauwerte auf Grund von georadiometrischen Faktoren wird als topographischer Effekt bzw. Neigung-AspektEffekt bezeichnet. Der topographische Effekt führt dazu, daß Gebiete mit gleichen Oberflächeneigenschaften im Fernerkundungsbild durch unterschiedliche Reflexionsverhältnisse anders abgebildet werden. Besonders in reliefreichen Gebieten sind die radiometrischen Unterschiede aufgrund der Topographie viel größer als die eigentlichen Reflektionsunterschiede von verschiedenen Objekten (GILLESPIE et al. 
1986, Gregory und Compagna 1990, Peng und PhiliP 1990, zitiert nach NiRALA und VenKATACHALAM, 2000). Zwar wird die Verschärfung von topographischen Merkmalen aufgrund unterschiedlicher Topographie in vielen geologischen Anwendungen als vorteilhaft betrachtet, jedoch sind die Wirkungen für die digitale Bildklassifizierung im allgemeinen unerwünscht (TEILLET et. al. 1982).

Eine oft benutzte Methode, um dieses Problem $\mathrm{zu}$ umgehen, ist eine nach Beleuchtungsklassen stratifizierte Klassifizierung. Bei dieser Methode werden Trainingsgebiete getrennt in jeder Beleuchtungsklasse genommen und anschließend werden die Klassen getrennt klassifiziert. Erst hinterher werden die einzelnen Klassen zusammengebracht.

Weiterhin wurden mehrere modelbasierte Methoden zur Korrektur des topographischen Effektes, welche als topographische Normalisierung bezeichnet werden, erarbeitet und angewandt. Die meisten Methoden beschrieben die reliefbedingten Grauwertvariationen der Oberfläche durch Beziehungen zwischen den lokalen Einstrahlungswinkel und den Sonnenzenitwinkel mit Hilfe eines digitalen Geländemodells (DGM).

Im folgenden wird die in dieser Untersuchung verwendete Methode der „Minnaert Korrektur“" kurz erläutert.

\section{Minnaert Korrektur}

In einem Lambertschen Strahlungsmodell wird die auf die Erdoberfläche einfallende Strahlung unabhängig von der Struktur der Oberfläche einheitlich in alle Richtungen reflektiert. Unter dieser Annahme beschreiben TEILLET et al. (1982) die Intensität der Strahlung, die auf einen Pixel fällt, als direkt proportional zur Illumination. Die Illumination wurde als der Kosinus des Zwischenwinkels der Sonneneinstrahlung und dem Flächennormal ( $\left.L_{H}\right)$ eines Pixels definiert (Abbildung 4.3). 


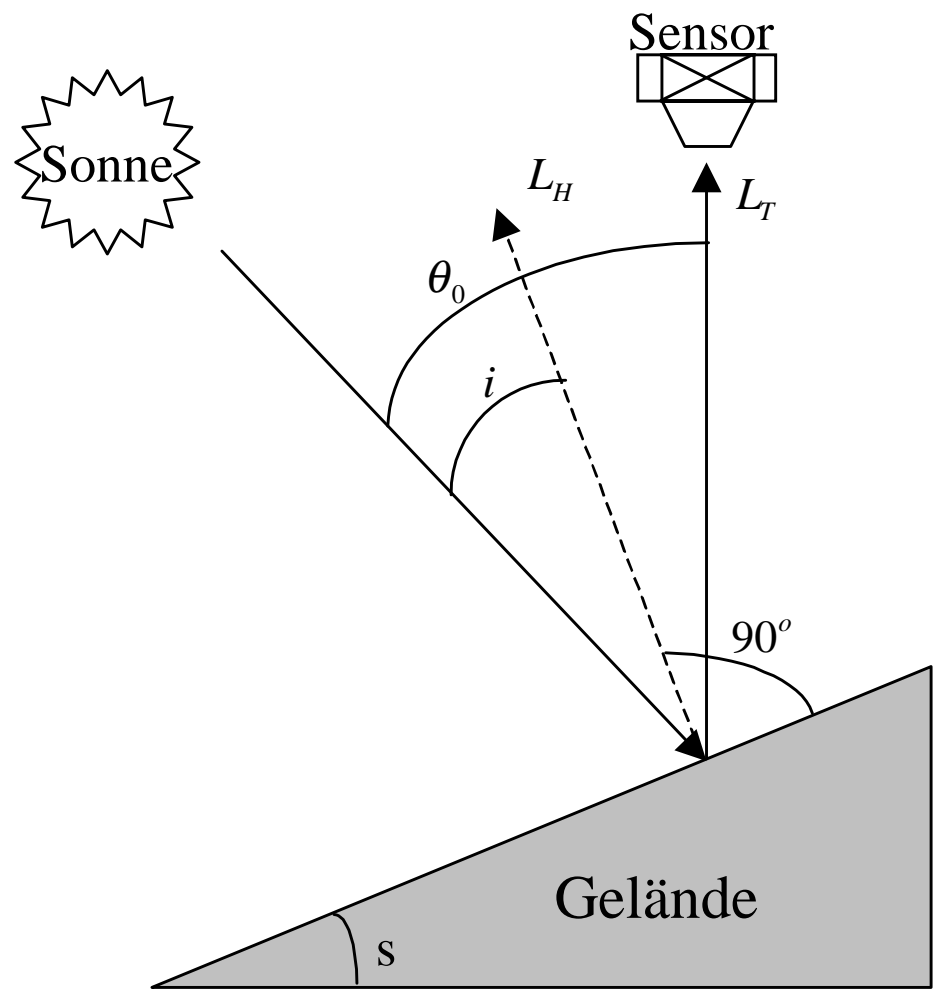

Abbildung 4.3:Schematische Darstellung der Illumination und Sonnenzenit $\theta_{0}$ ) (modifiziert nach JENSEN, 1996).

Die Illumination berechnet sich aus dem Sonnenzenit- und dem Sonnenazimutwinkel sowie der Hangneigung und der Exposition der Pixelflächen.

$$
\cos (i)=\cos \left(\theta_{0}\right) \cos (s)+\sin \left(\theta_{0}\right) \sin (s) \cos \left(\alpha_{0}-a\right)
$$

wobei $\theta_{0} \quad=$ Sonnenzenit

$\alpha_{0} \quad=$ Sonnenazimuth

$s \quad=$ Hangneigung

$a \quad=$ Exposition

Die Korrektur des topographischen Effektes wird dann nach der Formel

$$
L_{H}=L_{T} \frac{\cos \theta_{0}}{\cos _{i}}
$$

durchgeführt.

Wobei $L_{H}=$ Strahlung von einer horizontalen Fläche 
$L_{T} \quad=$ Strahlung von einer geneigten Fläche

sind.

Diese Methode ist als Kosinuskorrektur bekannt und ergibt eine Überkorrektur bei steilen und sonnenabgewandten Hängen, was auch von anderen Autoren berichtet wird (SMith et al. 1980, Civco 1989, BAyer 1990, Colby 1991, Meyer et al. 1993). Der Grund für die Überkorrektur liegt an der in diesem Verfahren angenommenen Lambertschen Richtungsverteilung der Reflexion.

Einer der Lösungsansätze zur Überkorrektur in der Kosinuskorrektur ist die Erweiterung der einfachen Kosinuskorrektur durch die sogenannte Minnaert-Konstante $k$ im Exponenten.

$$
L_{H}=L_{T}\left(\frac{\cos \theta_{0}}{\cos _{i}}\right)^{k}
$$

Mit der Einführung der Minnaert-Konstante $\mathrm{k}$ ist die Annahme der Lambertschen Reflektion nicht mehr gültig. Die Minnaert-Konstante wird empirisch durch lineare Regression zwischen den Reflexionswerten der Szene und den entsprechenden lokalen Einfallswinkeln ermittelt. Der Wert der Minnaert-Konstanten k entspricht der Steigung der Regressionsgeraden. Die Werte dieser Konstante k können zwischen 0 und 1 variieren. Sie geben an, inwiefern eine Oberfläche von den Lambertschen Reflektionsverhältnissen abweicht.

Zahlreiche Untersuchungen haben gezeigt, daß die Minnaert Korrektur für die topographischen Normalisierung vielversprechende Ergebnisse bringt (TEILLET et al. 1982, 1986, WoOdham and Grey 1987, Jones et al. 1988, Civeco 1989).

\subsection{Fusion der Satellitenbilder}

Eine Fusion von Bildern ist die Kombination von zwei oder mehreren Bildern, um ein neues Bild durch bestimmten Algorithmus zu erzeugen (POHL und GENDEREN 1998). Das Ziel von Bild-Fusionen ist es, unterschiedliche Informationsvorzüge miteinander zu integrieren. Ein fusioniertes Bild kann nicht nur mehr Information als jedes einzelne 
Bild, sondern auch verbesserte Interpretationsmöglichkeiten und damit verbesserte Ergebnisse liefern. Ein typisches Beispiel dafür ist u.a. die Fusion radiometrisch hoch auflösender multispektraler Satellitendaten mit geometrisch hoch auflösenden panchromatischen Daten. Allgemein sind die Fusionsverfahren in zwei Gruppen zu kategorisieren (POHL und GENDEREN 1998):

1) Farb-Transformationsverfahren

2) Statistische / Numerische Verfahren.

Statistische/Numerische Methoden werden auf Grundlage der Kanalstatistik einschließlich der Korrelation oder den Kovarianzen zwischen den Kanälen und Filtern entwickelt. Verfahren wie PCA, Regressionen und die Nutzung von Hochpaß Filtern oder Wavelet gehören zu dieser Gruppe. Anders als die Farb-Transformationsverfahren können bei Statistischen/Numerischen Verfahren beliebig viele multispektrale Kanäle gleichzeitig mit panchromatischen Daten fusioniert werden. Ein Nachteil dieser Methoden liegt u.a. darin, daß die Ergebnisse spezifisch jeweils für die bei der Fusion verwendeten Bilder sind. Unterschiedliche Größen von Bildern oder Kanalkombinationen liefern verschiedene Ergebnisse. Daher ist der direkte Vergleich zwischen den fusionierten Bildern nicht möglich.

Farb-Transformation Verfahren beziehen sich auf die Vor- und Rücktransformation von drei Bildkanälen im RGB-Farbraum in einen anderen Farbraum, z.B. IHS, HSV und YIQ. In diesem Abschnitt wird die IHS Transformation näher erläutert, welche in der vorliegenden Untersuchung verwendet wurde. Allgemeine und detaillierte Informationen zur „Daten Fusion“ sind z.B. bei MATHER(1999), JenSEN(1996), RICHARDS(1994) oder BÄHR und VÖGLTE(1991) zu finden.

\section{IHS Transformation}

Unter den farbbezogenen Verfahren ist die IHS Transformation die am häufigsten verwendete Methode (CARPer et. al, 1990., ShetTingara, 1992., Nunez et. al., 1999). Dabei werden drei Kanäle vom RGB-Farbsystem in ein IHS-Farbsystem transformiert, indem die Farbe mit den Begriffen Intensität (Intensity), Farbton (Hue) und Sättigung (Saturation) beschrieben wird. „Intensity“ ist die gesamte Helligkeit des Bildes und variiert zwischen 0 (schwarz) und 1 (weiß). „Hue“ ist der Begriff für die dominierende Wellenlänge eines Pixels. Sie wird in einem Farbkreis mit einem Wert zwischen 0 und 
360 dargestellt. Mit „Hue“ kann der Farbname z.B. Rot (0 und 360), Gelb (60), Grün (120), Cyan (180), Blau (240) und Magenta (300), bezeichnet werden. Die „Saturation“ stellt die Reinheit einer Farbe dar und nimmt einen Wert zwischen 0 und 1. Der Aufbau dieser Systeme ist zum Teil stark unterschiedlich, so wie die Werte I, H und S auch unterschiedlich definiert werden (KRAUS, 1990). Dies führt dazu, daß auch die Transformation zwischen IHS und RGB unterschiedlich definiert wird. Daher ist es nicht möglich, die IHS-Werte verschiedener Methoden direkt zu vergleichen.

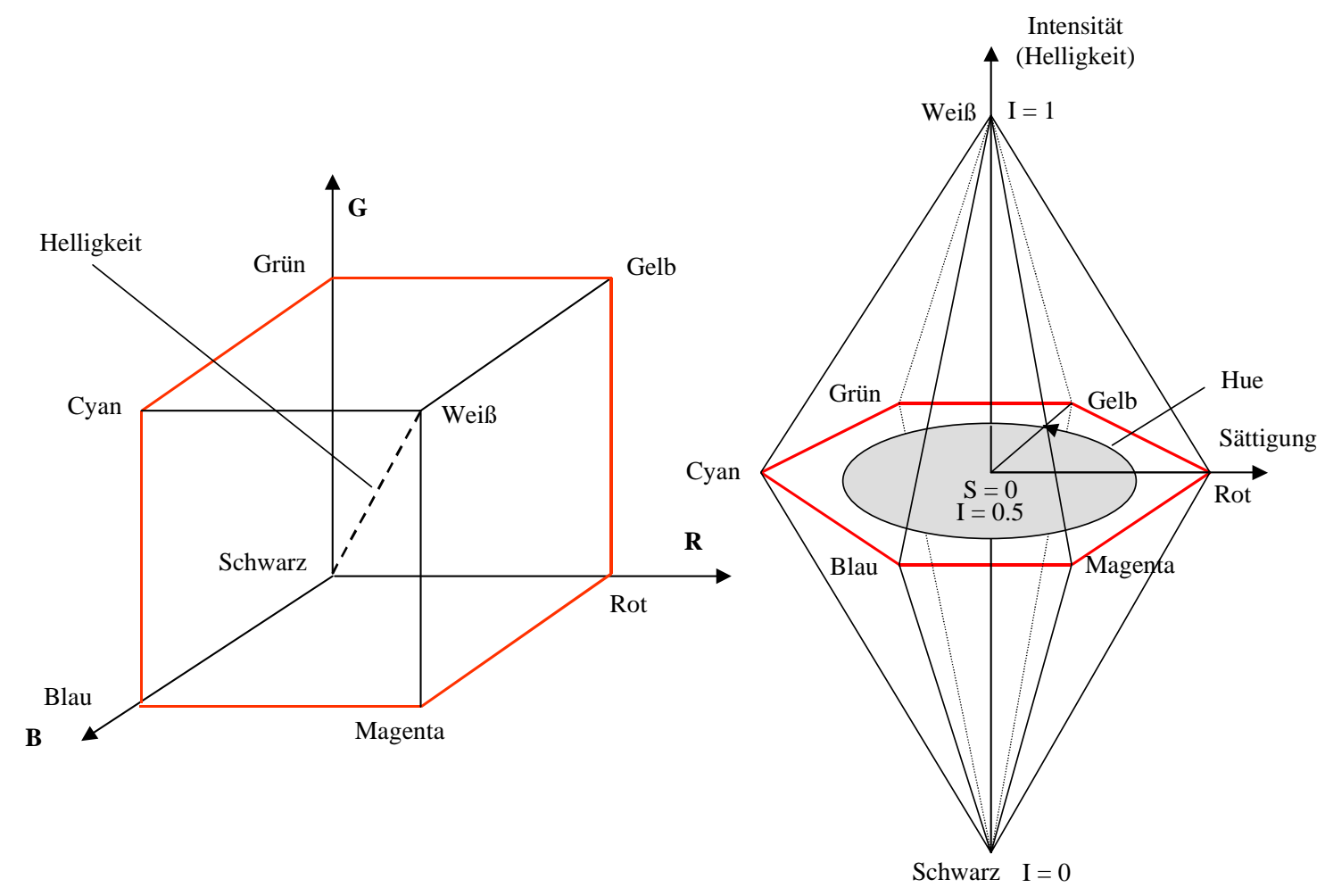

Abbildung 4.4:Schematische Darstellung der RGB Farbmodelle (links) und der IHSDoppel Hexcone Modelle (rechts)

Abbildung 4.4 stellt die Beziehung zwischen dem RGB-Farbmodell (links) und dem sog. „Doppel Hexcone“ Modell (rechts) für ein IHS-Farbsystem schematisch dar. Wie die Abbildung veranschaulicht, erhält man bei Betrachtung des RGB-Farbmodells in der Achse der Diagonalen von weiß nach schwarz ein „Doppel Hexcone“ Modell.

Für Transformationsformeln zwischen RGB Würfelkoordinaten und IHS Koordinaten sei auf Fachliteratur, z.B. KRAUS (1996) oder ERDAS (1999) verwiesen. 
Bei Durchführung der Fusion mit der Methode der IHS-Transformation werden zuerst die beiden Datensätze aufeinander rektifiziert. Dabei wird die geometrische Auflösung der multispektralen Daten an die der panchromatischen Daten angepaßt. Man wählt dann drei multispektrale Kanäle für die RGB-Darstellung aus und transformiert diese in das IHS-Farbsystem. Die transformierte Intensitätkomponente weist eine hohe Korrelation und Ähnlichkeit mit den hoch auflösenden panchromatischen Daten auf. Auf Grund dieser hohen Korrelation wird die Intensitätskomponente mit dem panchromatischen Datensatz ersetzt. Normalerweise werden dabei die hoch auflösenden panchromatischen Daten so modifiziert, daß die spektralen Unterschiede zur Intensitätskomponente minimiert werden. Dies kann durch eine Histogrammanpassung der panchromatischen Daten an die Intensitätskomponente erfolgen. Nach der Ersetzung wird eine inverse IHS-Transformation durchgeführt, so erhält man ein fusioniertes Bild, welches die Information von beiden Datensätzen enthält. Ein Nachteil dieser Methode besteht darin, daß die Transformation nur mit jeweils 3 Kanälen durchgeführt wird.

\subsection{Klassifikation multispektraler Satellitenbilder}

\subsection{Einführung}

Grundsätzlich gibt es zwei Methoden zur Auswertung multispektraler Satellitenbilder: die Visuelle Interpretation und die digitale Klassifizierung. Bei der visuellen Interpretation, wie man sie von der konventionellen Luftbildinterpretation her kennt, werden nicht nur die einzelnen Pixelwerte, sondern zusätzlich lokale und globale Informationen über das gesamte Bild wie Textur, Muster, Form und Große, Lage und Schatten mit herangezogen (BÄHR und VöGLTE 1991). Darüber hinaus kann der Interpreter seine gesamte Erfahrung, Ortskenntnis und weitere Informationen, wie z.B. topographische oder thematische Karten, falls verfügbar, in die Auswertung einbringen.

Eine digitale Klassifikation im Sinne der digitalen Bildverarbeitung ist ein computergestütztes automatisches Auswertungsverfahren, welches eigentlich ein Sortierungsprozeß von Pixeln aufgrund ihrer charakteristischen Merkmale in eine endliche Anzahl von individuellen Klassen ist (ERDAS 1999). 
Der Klassifikationsprozeß wird im allgemeinen in zwei Phasen, der Erstellung der Regeln (Training) und der Benennung (Labeling), durchgeführt (ERDAS 1999). Bei der Trainingsphase wird das Computer-System anhand von Pixelwerten erlernt, charakteristische Merkmale der Objekte im Bild zu erkennen und Kriterien zur Unterscheidung der einzelnen Objektklassen zu bilden. Dieser Vorgang kann entweder durch eine überwachte Methode oder eine unüberwachte Methode durchgeführt werden. Bei der überwachten Methode werden zuerst die gewünschten Objektklassen definiert und für jede Objektklasse ein oder mehrere Trainingsflächen (Referenzdaten) ausgewählt. Dabei sollen die Trainingsflächen homogen und für die Klasse repräsentativ sein. Aufgrund dieser bekannten Referenzdaten werden dann die Unterscheidungskriterien gebildet. Liegen keine Referenzdaten vor, so muß eine unüberwachte Methode verwendet werden. Mit Hilfe einer vorgegebenen Anzahl von Klassen benutzt die unüberwachte Methode die Informationen, die die Daten selbst tragen, für die Erkennung von Klassen und die Bildung ihrer Unterscheidungskriterien.

Die zweite Phase der Klassifikation ist die Zuweisung von jedem einzelnen Pixel zu einer bestimmten Klasse (Labeling). Erfolgte eine überwachte Klassifikationsmethode, dann wird der Pixel direkt zu der Klasse übertragen, die den Kriterien entspricht. Bei einem unüberwachten Trainingsverfahren sind die Ergebnisse einfach die vorgegebene Anzahl von Clustern. Die Pixel, die dem selben Cluster zugehören, haben ähnliche spektrale Merkmale. Es ist dann die Aufgabe des Analytikers, jedes Cluster einer bestimmten Klasse zuzuweisen.

\subsection{Pixel- und Segment basierte Klassifikation}

Es gibt eine ganze Reihe von Klassifikationsverfahren für Fernerkundungsdaten, die sich methodisch teilweise erheblich unterscheiden. Neben der Maximum Likelihood und der ISODATA(Iterative Self Organising Data Analysis Technique) Methode, die in der Literatur als überwachte- und unüberwachte Klassifikationsmethoden am häufigsten zur Anwendung kommen, sind die „k-Means Clustering“, die „Parallelepiped“ Klassifikation, die Wissensbasierte Methode, Fuzzylogik Algorithmen und das künstliche Neuronale Netze Verfahren als einige Beispiele von Klassifikationsmethoden zu nennen. Darüber hinaus gibt es auch zahlreiche Versuche zusätzliche Informationen wie z. B. Texturinformationen im Bezug auf Nachbarpixel oder aus externen Quellen, z.B. Geländehöheninformationen, in die Klassifikationsverfahren einzuschießen. 
Alle oben genannten Klassifikationsmethoden außer der Methode, die auf Texturinformationen beruht, sind Methoden, die individuelle Pixel aufgrund ihrer spektralen Eigenschaften zu einer der vordefinierten Klassen zuzuweisen. Diese Methoden, die als auch pixelbasierte Klassifikation bezeichnet werden, beruhen unter anderem auf der Annahme, daß alle Pixel in einem Bild überall die gleiche Erscheinungswahrscheinlichkeit haben. Aber in der realen Welt besteht eine höhere Wahrscheinlichkeit, das benachbarte Pixel die gleiche Klassenzugehörigkeit haben. Die pixelbasierten Klassifikationsmethoden berücksichtigen diese räumliche Abhängigkeit nicht. Besonders bei den räumlich hochauflösenden Satellitendaten wie IKONOS ist die räumliche Abhängigkeit jedoch größer als bei herkömmlichen Satellitendaten, welche eine geringere Auflösung haben.

Eine alternative Methode gegenüber der „Pixelbasierte Klassifikation“ ist die Einbeziehung der Segmentierung in das Klassifikationsverfahren (MATHER 1999). Nach PAL et al.(2000) ist die Segmentierung ein Verfahren, mit dem ein Bildraum in mehrere Regionen zerlegt wird, die sich untereinander nicht überlappen, homogen und sinnvoll sind. Das Wort sinnvoll ist von der Fragestellung abhängig. Nach der Segmentierung können statt Pixel die einzelnen Regionen (Segmente) klassifiziert werden. Diese Methode nennt man „Segmentbasierte Klassifikation“

Einige zusammenfassende und ausführliche Arbeiten zum Thema Klassifikationsmethoden finden sich beispielsweise bei JENSEN (1995), SCHOWENGERDT (1997) oder in MATHER(1999).

\subsection{Maximum Likelihood Klassifikation}

Die „Maximum Likelihood“ Methode ist, wie schon erwähnt, das in der Praxis am häufigsten angewendete überwachte Klassifikationsverfahren. Der Algorithmus setzt voraus, daß die Pixelwerte einer Klasse auf der multi-dimensionalen Ebene normalverteilt sind. Unter dieser Voraussetzung kann zu jeder Trainingsklasse unter Berücksichtigung der Mittelwerte und Kovarianzen eine Wahrscheinlichkeitsdichtefunktion berechnet werden. Zur Durchführung der Klassifikation wird für jedes zu klassifizierende Pixel seine Wahrscheinlichkeit, mit der es zu jeder einzelnen Klasse gehört, berechnet. Das Pixel wird dann zu der Klasse, für die es eine maximale Wahrscheinlichkeit besitzt, zugewiesen. Mit der Maximum 
Likelihood Methode wird die Klassenangehöhrigkeit eines Bildelementes nach SWAIN (1978) wie folgt bestimmt.

$$
x \in \omega_{i} \text { wenn } \mathrm{P}\left(x \mid \omega_{i}\right) \mathrm{P}\left(\omega_{i}\right)>\mathrm{P}\left(x \mid \omega_{j}\right) \mathrm{P}\left(\omega_{j}\right) \text { für alle } j \neq i
$$

wobei $\mathrm{P}\left(x \mid \omega_{i}\right), \quad i=1, \ldots, k$, die Diskriminanzfunktionen sind, die aus den Trainingsflächen über die Formel

$$
P\left(x \mid \omega_{i}\right)=\ln P\left(\omega_{i}\right)-\left[0,5 \cdot \ln \mid \operatorname{Cov}_{i}\right]-\left[0,5 \cdot\left(x-\bar{x}_{i}\right)^{\prime} \operatorname{Cov}_{i}^{-1}\left(x-\bar{x}_{i}\right)\right]
$$

geschätzt werden.

$P\left(\omega_{i}\right)$ ist die apriori Wahrscheinlichkeit der Klasse ' $i$ '. Wenn die apriorischen Wahrscheinlichkeiten unbekannt sind oder aus anderen Informationsquellen nicht geschätzt werden können, was oft in praktischen Anwendungen der Fall ist, wird der Wert '1' für alle $P\left(\omega_{i}\right)$ gesetzt (ERDAS, 1999; JENSEN, 1996).

Obwohl in vielen praktischen Fällen die Voraussetzung der Normalverteilung nicht erfüllt ist, liefert der ML Klassifikator akzeptable Ergebnisse und hat sich in vielen Untersuchungen bewährt.

\subsection{Segment basierte Klassifikation}

\subsection{Bildsegmentierung}

Segmentierung ist der ersten Schritt für eine segmentbasierte Klassifikation. Bei der Segmentierung wird das Bild in zahlreiche homogene Teilflächen eingeteilt, welche anschließend die Basiseinheiten für die Klassifikation bilden. Aus der Literatur sind zahlreiche Segmentierungsverfahren bekannt. NIEMEYER (2001) unterscheidet Segmentierungsverfahren mit folgenden Ansätzen:

- Mit Hilfe von Histogrammanalysen oder Clusterverfahren können die Daten in Segmente geordnet werden (DYLYAKARN et al. 1999, PUZICHA et al. 1999).

- Segmentierungsverfahren auf der Grundlage der Kantendetektion suchen anhand verschiedener Kriterien die Grenzen zwischen homogenen Bildbereichen (FARAG 1992, FJORTOFT et al. 1998). $\mathrm{Zu}$ diesen Techniken werden auch morphologische Segmentierungsverfahren, wie die sogenannte Wasserscheidentransformation 
(Watershed Transformation) gezählt (SOILLE 1998, PESARI und KANELLOPOULOS 1999, BEUCHER und MEYER 1993, MEYER und BEUCHER 1990).

- Sogenannte „Split“-Techniken teilen entsprechend der definierten Homogenitätskriterien die Bilddaten in Segmente (KING 1997).

- Das Flächenwachstum (Region Growing) erfolgt ausgehend von der kleinsten Bildeinheit unter Berücksichtigung von Homogenitätskriterien (COOK et al. 1994, FELZENSWALB und HutTENLOCHER 1998, MiliaResis 1999).

- Sogenannte „Split and Merge“-Algorithmen verbinden „Split-,, und „Region Growing“ Techniken (vgl. HARALICK und SHAPIRO 1992).

- Hybride Techniken kombinieren verschiedene Verfahren, wie „Clustering“ oder Kantendetektion und „Region Growing“ (FRERY et al. 1999, KARTIKEYAN et al. 1998, Tilton 1996, Tilton 1998).

- Segmentierungsverfahren mit Hilfe Neuronaler Netze stellen COOLEY und COOLEY (1999) sowie KURGÖLLÜS und SANKUR (1999) vor.

- Textursegmentierungsverfahren arbeiten auf der Grundlage der sogenannten Haralick-Parameter (HARALICK et al. 1973, HARALICK und SHAPIRO 1992), der Markov- Zufallsfelder (Markov Random Fields) (Li 1995, WANG 1999), der fraktalen Dimension (CHAUdHURI und SARKAR 1995, Keller und CHEN 1989, MÜSSIGMANN 1992) oder mit Hilfe von spektralen Filtertechniken (HoFFMANN et al. 1998, JAIN und FARroKhNIA 1991, LiU und PICKARD 1996). Die Gruppierung in homogene Segmente erfolgt zumeist mit Hilfe der Kostenfunktion (JAIN und FARROKHNIA 1991, HoFMANN et al. 1998, PUZICHA und BUHMANN 1999).

- Regelbasierte Verfahren verwenden ein wissensbasiertes System für die Segmentierung (GorTE 1998, KunZ et al. 1998, McKeOwn et al. 1988, SHANDLEY et al. 1996, TönJes 1999, WoOdCOCK und HARWARd 1992, XUAN und AdAH 1996).

- Multiskalige Segmentierungsverfahren basieren beispielsweise auf dynamischen Zuweisungsbäumen (Label Tree) (REHRAUER et al. 1998) oder Fuzzy ClusteringTechniken (ThITIMAJSHIMA und CHUMSAMRONG 1999). 
Für einen guten Überblick zum Thema Segmentierung ist auf die Arbeit von HARALICK und Shapiro (1992), SAgERER und Niemann (1997), SchnÖrr (1997) und SonKa et al. (1998) zu verweisen.

Im Rahmen der vorliegenden Untersuchung wurde die Software „eCognition“ zur Segmentierung verwendet.

\subsection{2 ,Multiresolution Segmentation“ in eCognition}

„eCognition“ ist eine von der Firma „Definiens Imaging“ entwickelte BildanalyseSoftware, die eine Klassifikation nach einer segmentbasierten Methode durchführt. Bei einer segmentbasierten Klassifikation spielt die Qualität der Segmentation eine entscheidende Rolle, weil die einzelnen Segmente die Eingangseinheit der Klassifikation sind. Die in der Software „eCognition“ implementierte „Multiresolution Segmentation“ kann als eine „Region Growing Methode“ angesehen werden. Nach BAATZ und SCHÄPE (2000) fängt die Prozedur zuerst auf der Pixelebene an, d.h. jedes einzelne Pixel wird als eigenes Segment behandelt. Dann werden die Segmente mit den Nachbarsegmenten zusammen fusioniert und bilden neue Segmente. Diese Prozedur wird so lange durchgeführt, bis ein vorgegebener minimaler Grenzwert nicht überschritten wird.

\subsubsection{Definition der Heterogenität}

Die Heterogenität wurde in eCognition in zwei Kategorien, der spektralen- und der räumlichen Heterogenität, definiert und kombiniert verwendet.

Die spektrale Heterogenität, in eCognition als „Color“ bezeichnet, ist die Summe der gewichteten Standardabweichungen der Spektralwerte in einem Segment (DEFINIENS IMAGING, 2001).

$$
h_{\text {color }}=\sum_{c} \omega_{c} \sigma_{c}
$$

wobei $\omega$ : Gewichtsfaktor

$\sigma:$ Standardabweichung

c : Einzelner Kanal

In vielen Fällen ist jedoch die alleinige Minimierung der spektralen Heterogenität nicht zielführend, da dadurch weitverzweigte Segmente, bzw. Segmente mit sehr hoher 
fraktaler Dimension entstehen (siehe. Abbildung 4.5). Um diese Probleme zu umgehen wurde die räumliche Heterogenität, in eCognition „Shape“ genannt, als ein weiteres Kriterium eingeführt. Die räumliche Heterogenität ist wiederum über zwei Merkmale definiert: die Glattheit (Smoothness) und die Kompaktheit (Compactness).

Die „Smoothness“ ist das Verhältnis zwischen der Länge der Umrißlinie $L$ eines Segmentes und der Länge der kürzest möglichen „Bounding Box“ Linie $B$. Die „Bounding Box“ ist ein Rechteck parallel zum Bildraster und schließt das Segment genau ein.

$$
h_{\text {Smooth }}=\frac{L}{B}
$$

Die „Compactness“ eines Segments ist durch die Länge der Umrißlinie $L$ geteilt durch die Quadratwurzel der Anzahl von Pixeln $(N)$ des Segmentes beschrieben.

$$
h_{\text {Compact }}=\frac{L}{\sqrt{N}}
$$

Die drei Heterogenitäten zusammengefaßt, wobei die einzelnen Werte zu gewichteten sind, bilden die Heterogenität eines Segmentes.

$$
h=w_{\text {Color }} h_{\text {Color }}+w_{\text {Shape }}\left(w_{\text {Smooth }} h_{\text {Smooth }}+w_{\text {Compact }} h_{\text {Compact }}\right)
$$

wobei $w_{\text {Color }}+w_{\text {Shape }}=1$

$$
w_{\text {Smooth }}+w_{\text {Compact }}=1 \text { sind. }
$$

\subsubsection{Scale Parameter}

Der minimale Grenzewert, der bei der Segmentierung nicht überschritten werden darf, wird in „eCognition“ als Maßstabsparameter (Scale Parameter) genannt. Der „Scale Parameter" ist die Differenz der kumulativen Heterogenität zwischen dem Vor und Nach einer Fusionierung von zwei Segmenten. Dabei werden die einzelnen Kanäle und die Heterogenität gewichtet eingesetzt.

BAATZ und SCHÄPE (2000) haben den „Scale Parameter“ wie folgt formuliert: 


$$
h_{\text {diff }}=\sum_{c} \omega_{c}\left[n_{1}\left(\bar{h}_{m c}-h_{1 c}\right)+n_{2}\left(\bar{h}_{m c}-h_{2 c}\right)\right]
$$

wobei :

$h_{\text {diff }}:$ Scale Parameter, Differenz der Heterogenität

$h_{i c}$ : Heterogenität der Segment, $i=1,2$

$\bar{h}_{m c}$ : Mittelwerte der Heterogenität von zwei Segmente

$\omega$ : Gewichtsfaktor

$n$ : Pixelzahl in einem Segment

c : Einzelner Kanal

\subsubsection{Bestimmung der Parametergröße}

Die Bestimmung der Parametergroße bei der „Multiresolution Segmentation“ ist eine heuristische Optimierungsprozedur. Zur Bestimmung der Parametergröße bietet eCognition die Möglichkeit, jede Heterogenität einzeln zu gewichten. Mit einem hohen Gewicht auf dem „Shape Parameter“ und der „Compactness“ erhält man ein rundförmiges Segment.

Der „Scale Parameter“ bestimmt dann die Größe des Segmentes. Hierbei ist zu beachten, daß der „Scale Parameter“ nicht mit einem herkömmlichen Bildmaßstab direkt vergleichbar ist. Er ist ein Schwellenwert, der ein Maß für die maximal erlaubte Heterogenität eines Segmentes ist. Mit einem kleinen „Scale Parameter“ wird eine kleine maximale Heterogenität erlaubt und es werden kleinere Segmente im Vergleich zu einem größeren „Scale Parameter“ gebildet. Da der „Scale Parameter“ über alle Kanäle aufsummiert wird, sollte man auch die Anzahl der verwendeten Kanälen beachten. Ein „Scale Parameter“ von 90 bei der Verwendung von drei Kanälen entspricht z.B. in etwa einem Wert von 30 für einen Kanal oder ist vergleichbar mit einem „Scale Parameter“ von 180 bei der Verwendung von sechs Kanälen.

Die Auswahl der optimalen Kombination der Parameter ist vom Zweck der Segmentierung und von den verwendeten Daten abhängig, eine allgemeingültige Lösung gibt es nicht. In Abbildung 4.5 wurde die Segmentbildung mit verschiedenen Kombinationen der Parameter beispielhaft dargestellt. 


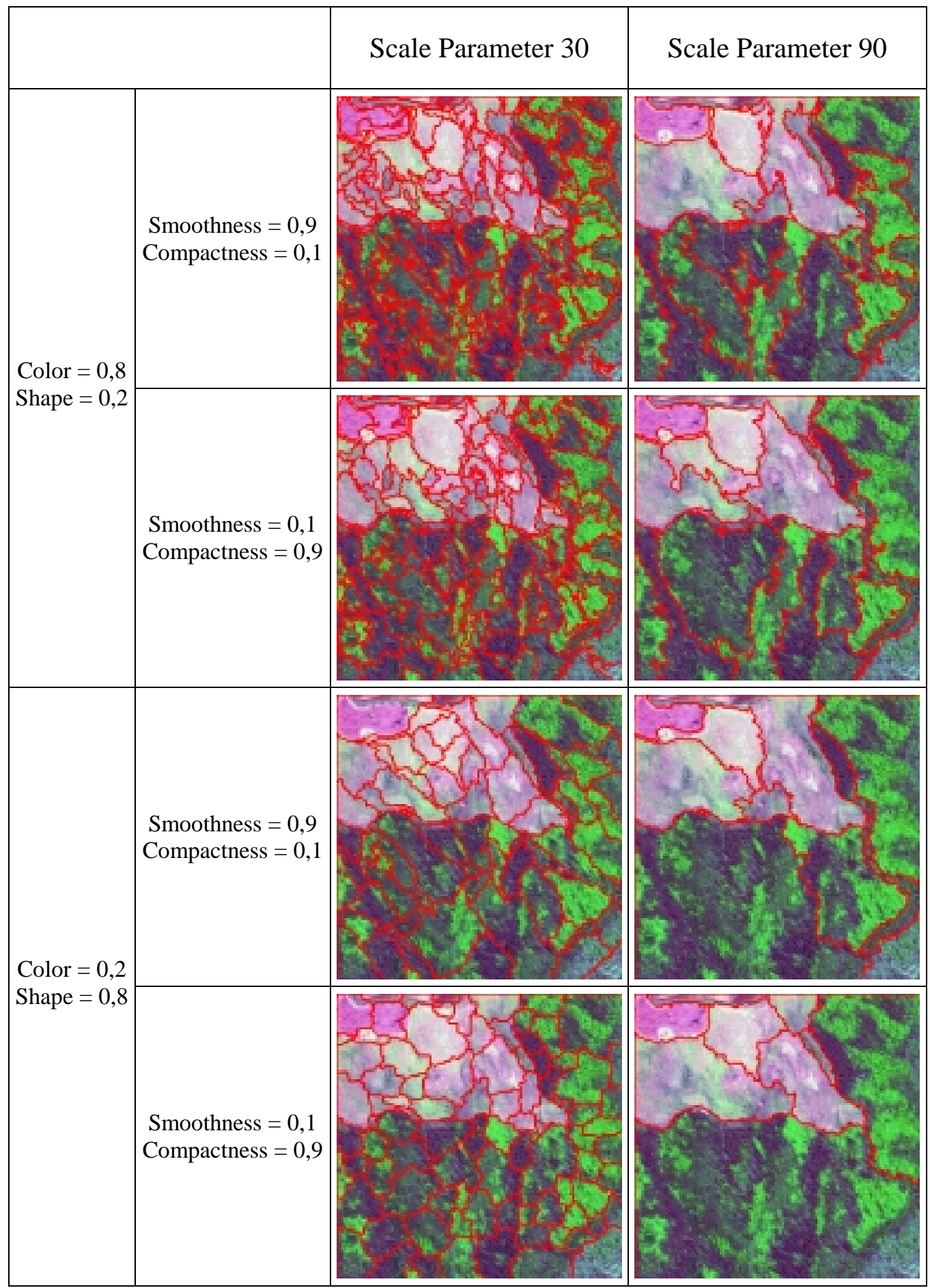

Abbildung 4.5: Beispiele der Segmentbildung nach verschiedener Kombination der Parameter. Dargestellt wurden die Ergebnisse anhand einer fusionierten IKONOS Szene. 


\subsubsection{Hierarchische Netz der Segment}

Mit Vergrößerung des „Scale Parameters“ ist es möglich, größere Segmente zu bilden. Versucht man ein bereits segmentiertes Bild mit einem größeren „Scale Parameter“ noch mal zu segmentieren, dann ergibt sich eine zusätzliche Ebene mit größeren Segmenten. Die einzelen Segmente der ersten Segmentation sind dabei Eingangselement der zweiten Segmentation. Da in eCognition ein „Region Growing Algorithmus“ für die Segmentation benutzt wird, liegen die Grenzen der Segmente im oberen Level (Superobjects) immer genau auf die Grenzen der unteren Level (Subobjects). Es entsteht dadurch eine Objekthierarchie, bei der jedes Segment seine Sub- und Super-Segmente kennt.

Eine neue Segmentierung kann zwischen beliebigen Leveln durchgeführt werden. Dabei wird jeweils ein neuer Level erzeugt, der auf den Segmenten seines direkten unteren Level basiert. Jedoch werden die neuen Segmente von den Grenzen der Superobjekte beschränkt. Die Grenze der Superobjekte können nicht vermischt werden. In Abbildung 4.6 wird dieses Prinzip veranschaulicht.

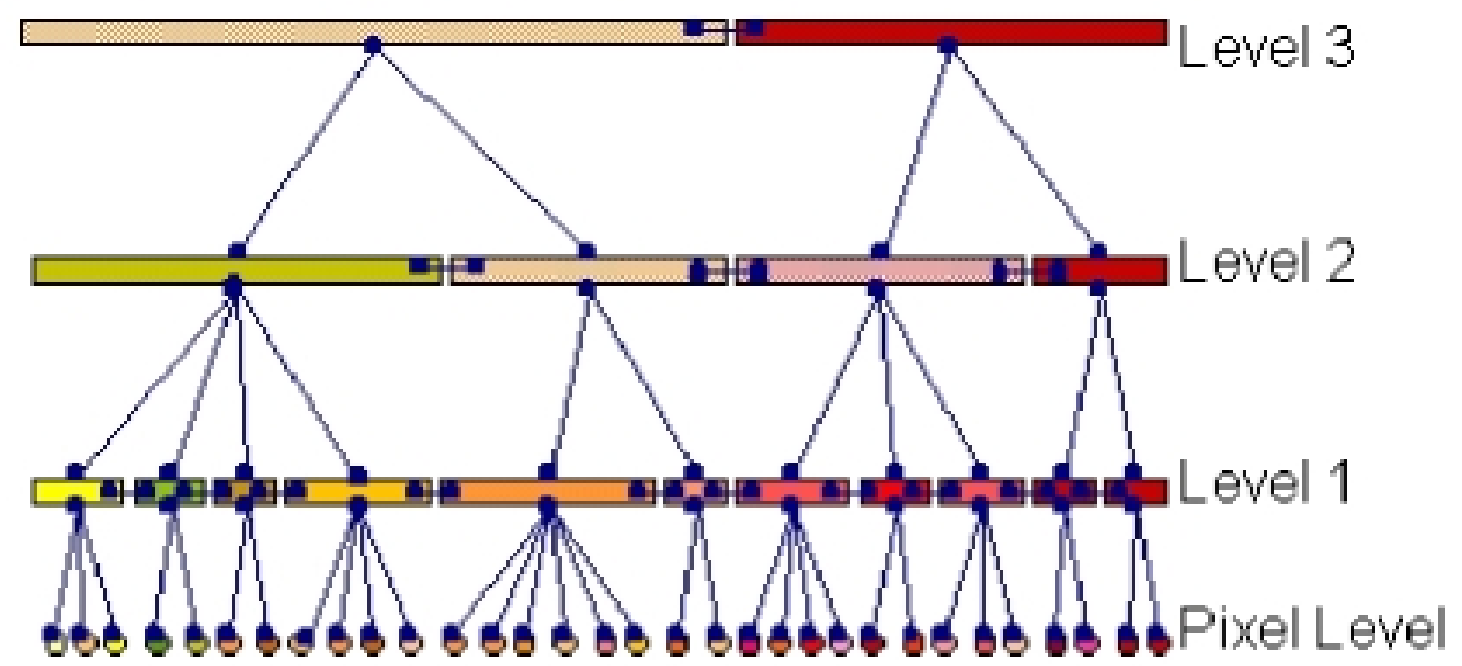

Abbildung 4.6: Schematische Dargestellung einer Objekthierarchie mit drei Segmentierungsebenen (DEFINIENS IMAGING, 2001) 
Das hierarchische Netz der Bildsegmente stellt neue Analysemöglichkeiten digitaler Bilder zur Verfügung (DEFINIENS IMAGING, 2001) :

- Strukturen von unterschiedlichen Maßstäben können gleichzeitig dargestellt und für die Klassifikation neue Variablen, die auf den Verhältnissen zwischen den Segmenten basieren, mit herangezogen werden.

- Daten aus unterschiedlicher Herkunft, z.B. eine thematischen Karte, können für die Segmentation in einem beliebigen Level herangezogen werden.

- Die Form der Bildsegmente kann durch eine Umgruppierung der Segmente im unteren Level korrigiert werden.

\subsection{Zuweisung der Segmente in eine Klasse (Labelling)}

\subsubsection{Klassenzuweisung nach dem Mehrheitsprinzip (Mehrheitszuweisung)}

Nach der Segmentierung kann aufgrund der Ergebnisse der pixelbasierten Klassifikation jedes einzelne Segment eindeutig in eine Klasse zugeordnet werden. Hierbei wird das jeweilige Segment derjenigen Klasse zugeordnet, welche am häufigsten dem im Segment vorkommenden Klasse zugewiesen wurde. Treten beispielsweise innerhalb eines Segmentes zu 75\% Pixel der Klasse Waldflächen und zu 25\% Pixel der Klasse Nichtwald auf, so wird das Segment der Klasse Waldflächen zugewiesen.

\subsubsection{Segmentbasierte „Maximum Likelihood“-Klassifikation}

Die Methode der „Maximum Likelihood“-Klassifikation kann auch anstatt mit Pixeln auf der Segmentebene durchgeführt werden. Das einzelne Segment ist dann die Grundeinheit der Klassifikation. Dabei werden die Merkmale zur Klassifikation bezogen auf Segmente gewonnen. In der vorliegenden Untersuchung wurden die segmentweise hergeleiteten Mittelwerte und die Standardabweichungen von Grauwerten einzelner Kanäle mit in die Klassifikation einbezogen. 


\subsection{Genauigkeitsüberprüfung}

Auswertungsergebnisse von Fernerkundungsdaten können sowohl als eine Grundlage für politische und wirtschaftliche Entscheidungen, als auch Ausgangsdaten für weitere Analysen mit oder ohne anderen Daten dienen. Für den potentiellen Benutzer ist es notwendig $\mathrm{zu}$ beurteilen, ob die Genauigkeit der verwendeten Daten für seine Anwendungszwecke ausreicht. Vor allem bei den Benutzern, die eine integrierte Nutzung mit einem GIS vorhaben, ist die Genauigkeit einer thematischen Karte von großer Bedeutung. Daher ist die Genauigkeitsüberprüfung ein wichtiger Schritt bei der Analyse der Fernerkundungsdaten.

Die Genauigkeit eines raumbezogenen Datensatzes wird definiert als die Nähe der Ergebnisse von Beobachtungen, Berechnungen und Schätzungen zu den wahren Werten oder den als wahr angenommenen Werten (USGS, 1990). Bei einer thematischen Karte, die durch Klassifikation von Satellitendaten entsteht, kann die Genauigkeit in zwei Gesichtpunkten, geometrisch und thematisch, beurteilt werden (Janssen und Wel 1994).

\subsection{Lage Genauigkeit}

\section{RMS Fehler}

Die Lagegenauigkeit bezieht sich auf das Georeferenzierungsverfahren, das bereits im Abschnitt 4.21 erläutert wurde. Nach Abschluß der Georeferenzierung werden die Residuals in X- und Y-Richtung hergeleitet. Diese werden dann für die Rechnung des mittleren quadratischen Fehlers (RMSE: Root Mean Square Error) verwendet. Der RMS Fehler in Richtung X wird berechnet durch

$$
R M S E_{x}=\sqrt{\frac{1}{n} \sum_{i=1}^{n}\left(\delta_{x i}\right)^{2}}
$$

wobei, $\delta_{x i}$ der Residual des $i$-te GCPs in Richtung X und $n$ die Anzahl des GCPs sind.

Der RMS Fehler in Richtung Y kann mit der selben Formel berechnet werden, wobei dann die entsprechenden Residuen in Y-Richtung verwendet werden. Anschließend werden die $R M S E_{x}$ und $R M S E_{y}$ für die Berechnung eines planimetrischen RMS Fehlers $\left(R M S E_{x y}\right)$ nach der Formel 


$$
R M S E_{x y}=\sqrt{R M S E_{x}^{2}+R M S E_{y}^{2}} \text { herangezogen. }
$$

\section{Circular Error $90 \%$ : CE90}

Die Lagegenauigkeit kann auch anhand von „Circular Error“ mit Vertrauensbereich angegeben werden. „Circular Error $90 \%$ \% (CE90) z.B. bedeutet, alle Punkte im Bild mit 90\% Wahrscheinlichkeit innerhalb einer angegeben Entfernung zu ihrer wahren Lageposition liegen.

Wenn die RMS Fehler in X- und Y-Richtung normal verteilt und gleich groß in beide Richtungen sind, kann CE90 aus dem RMS Fehler mit dem Faktor 2.146 berechnet werden. (Greenwalt und Schultz, 1968 zitiert nach FGDC, 1998).

$$
\begin{aligned}
\text { CE90 } & =2.146 * \text { RMSEx }=2.146 * \text { RMSEy } \\
& =2.146 * \text { RMSExy } / 1.4142 \\
& =1.5175 * \text { RMSExy }
\end{aligned}
$$

\subsection{Thematische Genauigkeit}

Die Genauigkeitsüberprüfung einer thematischen Karte, die durch Klassifikation einer Satellitenaufzeichnung entsteht, erfolgt durch Vergleich mit Referenzdaten (Ground Truth). Dabei beeinflussen mehrere Faktoren die Ergebnisse der Genauigkeitsüberprüfung. Die Auswahl der Referenzdaten (z. B. Quelle der Referenzdaten, Stichprobenverfahren und Größe der Stichprobe), das Klassifikationsschema (z.B. Anzahl und Definition der Klasse, Minimale Größe der Klasse) und die Analysemethode der Genauigkeitsüberprüfung können als Einflußfaktoren genannt werden. Für eine ausführliche Diskussion über dieses Thema sei auf Congalton (1998, 1991), Jensen (1996), STEHMAn und CZAPlewsKi (1998), StehmAn (1999), SMits et al. (1999), und SMith et al. (2002) verwiesen.

In diesem Abschnitt wird die in der vorliegenden Untersuchung verwendete Analysemethode mit einer Fehlermatrix näher erläutert. Die Fehlermatrix-Methode wurde von mehreren Autoren für die Genauigkeitsüberprüfung thematischer Karten empfohlen und ist inzwischen ein Standardverfahren geworden (CONGALTON 1991, JANSSEN und VAN DER Wel 1994, STEHMAN 1999, SMits et al. 1999, Zhu et al. 2000). 


\subsection{Fehlermatrix}

In einer Fehlermatrix werden die Referenzdaten auf der horizontalen Achse und die klassifizierten Ergebnisse auf der vertikalen Achse dargestellt. In die einzelnen Zellen der Fehlermatrix können entweder die Anzahl der Pixel, die Flächengröße einer bestimmten Klasse, oder auch prozentuale Anteile (normalisierte Zahlen) eingetragen werden. Im folgenden wird dies mit Pixel-Angaben erläutert. In der Diagonalen einer Fehlermatrix wird die Anzahl richtig klassifizierter Pixel und in den restlichen Zellen die Anzahl der falsch klassifizierten Pixel eingetragen. In der Tabelle 4.2 ist die Schreibweise einer Fehlermatrix für eine Klassifikation mit $q$ Klassen dargestellt, wobei $x_{i j}$ die Anzahl der Pixel darstellt, welche zur klassifizierten Klasse $i$ und Referenzklasse $j$ gehört. Weiterhin stellt der Ausdruck $X_{i+}=\sum_{j=1}^{q} x_{i j}$ die Summe der Zeile $i, \quad X_{+j}=$ $\sum_{i=1}^{q} x_{i j}$ die Summe der Spalte und die $N$ den Gesamtumfang der zu überprüfenden Pixel dar.

Tabelle 4.2: $\quad$ Aufbau einer Fehlermatrix für eine Klassifikation mit q Klassen. Siehe im Text zur Erläuterung des Symbols (modifiziert nach STEHMAN und CZAPLEWSKI, 1998).

\begin{tabular}{|c|c|c|c|c|c|c|}
\hline & \multicolumn{6}{|c|}{ Referenzdaten } \\
\hline \multirow{8}{*}{ 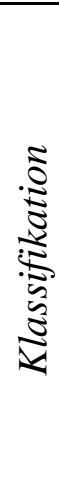 } & Klasse & 1 & 2 & $\ldots$ & $q$ & Summe \\
\hline & 1 & $x_{11}$ & $x_{12}$ & $\cdots$ & $x_{1 q}$ & $X_{1+}$ \\
\hline & 2 & $x_{21}$ & $x_{22}$ & $\ldots$ & $x_{2 q}$ & $X_{2+}$ \\
\hline & . & . & . & $\ldots$ & . & . \\
\hline & $\cdot$ & $\cdot$ & · & $\cdots$ & $\cdot$ & - \\
\hline & $\cdot$ & $\cdot$ & · & $\cdots$ & $\cdot$ & . \\
\hline & $q$ & $x_{q 1}$ & $x_{q 2}$ & $\cdots$ & $x_{q q}$ & $X_{q+}$ \\
\hline & Summe & $X_{+1}$ & $X_{+2}$ & $\ldots$ & $X_{+q}$ & $N$ \\
\hline
\end{tabular}

Prinzipiell erlaubt eine Fehlermatrix sowohl beschreibende als auch analytische Statistiken (SMITS et al. 1999). Beschreibende Verfahren sind relativ einfach und schließen die Herleitung der Gesamtgenauigkeit und der klassenspezifischen Genauigkeit ein. Die Gesamtgenauigkeit („overall accuracy“) ist das Verhältnis zwischen der Summe der Hauptdiagonalen d.h. Anzahl der richtig klassifizierten Pixel 
und der Gesamtanzahl der zu überprüfenden Pixel. Die Gesamtgenauigk errechnet durch:

$$
P_{\text {all }}=\frac{\sum_{i=j=1}^{q} x_{i j}}{N}
$$

Da die Gesamtgenauigkeit die zufälligen Übereinstimmungen nicht berücksichtigt und ungünstige Verteilungen von einzelnen Klassenübereinstimmungen nicht erfaßt, ist sie wenig aussagekräftig und insbesondere für den Vergleich verschiedener Klassifikationsabläufe ungeeignet (DARVISHSEFAT 1995).

Die klassenspezifische Genauigkeit gibt Auskunft darüber, wie gut eine Klasse klassifiziert worden ist. Sie kann auf zwei Arten aufgefaßt werden (STORY und Congalton 1986). Die Benutzergenauigkeit (,user's accuracy“) ist interessiert an der Zuverlässigkeit einer klassifizierten thematischen Karte. Sie ist auf den Zeilen dargestellt und zeigt, ob ein Pixel, welches zur Klasse $i$ klassifiziert worden ist, auch in der Wirklichkeit zu der Klasse gehört. Damit stellt sie die Wahrscheinlichkeit der Fehler 1. Art (,commission error"), d.h.die irrtümlich zu einer anderen Klasse zugewiesenen Wahrscheinlichkeit, dar. Die Benutzergenauigkeit für die Klasse $i$ wird mit folgender Formel berechnet:

$$
P_{U i}=\frac{x_{i i}}{X_{i+}}
$$

Die Genauigkeit des Herstellers (,producer's accuracy“) wird auf den Spalten dargestellt. Sie berücksichtigt die fälschlicherweise zur bestimmten Klasse $i$ zugeordneten Pixels nicht und zeigt die Wahrscheinlichkeit, mit der ein Pixel in den Referenzdaten richtig klassifiziert worden ist. Damit beschreibt die Herstellergenauigkeit die Fehler 2. Art (,ommission error“) und wird nach folgender Formel berechnet:

$$
P_{P j}=\frac{x_{j j}}{X_{+j}}
$$




\subsection{Kappakoeffizient}

Mit einer Fehlermatrix lassen sich durch analytische statistische Verfahren noch weitere Genauigkeitsmerkmale herleiten. Eine der Auswertungstechniken ist das „Diskrete multivariable Verfahren“ zur statistischen Prüfung der Klassifikationsgenauigkeit. Der Kappakoeffizient, welcher von CONGALTON et al. (1983) als ein geeignetes Maß dafür vorgestellt wurde, ist ein häufig verwendetes multivariables Verfahren für die Genauigkeitsüberprüfung. Für die Schätzung des Kappakoeffizienten wird die sogenannte KHAT Statistik herangezogen (CONGALTON und Mead 1983, STEHMAN 1996). Sie eliminiert die sogenannte Zufalls-Übereinstimmung in einer Gesamtgenauigkeit und gibt damit ein Maß, ob eine Fehlermatrix sich vom zufälliges Ergebnis signifikant unterscheidet. Der Kappakoeffizient kann auch benutzt werden, um unterschiedliche Fehlermatrizen aus verschiedenen Klassifikationsmethoden miteinander zu vergleichen und zu beurteilen, ob ein Ergebnis besser als das andere ist.

Die KHAT Statistik kann über

$$
\hat{K}=\frac{N \sum_{i=j=1}^{q} x_{i j}-\sum_{i=j=1}^{q}\left(x_{i+} x_{+j}\right)}{N^{2}-\sum_{i=j=1}^{q}\left(x_{i+} x_{+j}\right)}
$$

berechnet werden (CONGALTON 1991).

Der Kappakoeffizient variiert zwischen 0 und 1, wobei der Wert 1 die volle Übereinstimmung mit den Referenzdaten bedeutet.

\subsection{Veränderungsnachweis}

Die Erfassung der Veränderung (Change detection) ist der Prozeß der Identifizierung der Zustandsdifferenzen eines Objektes bzw. Phänomens in unterschiedlichen Zeitpunkten (SINGH, 1989). Die grundliegende Idee des Veränderungsnachweises ist die Annahme, daß sich Änderungen in der Landbedeckung in Änderungen der Merkmale eines Fernerkundungsdatensatzes wiederspiegeln. Diese müssen größer sein als die Änderungen, die durch andere Faktoren, wie z.B. atmosphärische Bedingungen, Bodenfeuchtigkeit und Sonneneinstrahlungswinkel verursacht werden. Außerdem haben folgende Faktoren nachteilige Auswirkungen bei einem Veränderungsnachweis, 
weshalb deren Wirkungen möglichst weitgehend ausgeschlossen werden sollen: die Kalibrierung des Sensors (im Falle der Verwendung verschiedener Aufnahmesysteme), die geometrische Korrektur oder der phänologischer Zustand der Vegetation.

Die Ergebnisse eines Veränderungsnachweises und damit verbundende Verfahrensmethoden wurden u.a. für Zwecke der forstlichen Behandlung (HAME et al. 1998, COPPIN et al. 2001), zur Überwachung und Erfassung des Waldzustandes (AKÇA et al. 1996, Collins und WoODCOCK 1996), und zur Überwachung, Kartierung und Modellierung von Waldschäden (MUCHONEY und HAACK 1994, GARCÍA-HARO et al. 2001, LEBLON et al. 2001) herangezogen. Außer forstlichen Anwendungen wurden Veränderungsnachweis-Methoden beim Monitoring der Vegetations- und Landnutzungsveränderungen (ZERDA 1998), Monitorring und der Modellierung der Urbanisierung (RADBERGER 2001), der Analyse der Landnutzungsentwicklung und deren Einfluß auf die nachhaltige Entwicklung (CHEN 2002), und zur Überprüfung der topographischen Karten (METTERNICHT 1999) eingesetzt.

Zahlreiche Methoden wurden für Veränderungsnachweise entwickelt, um die Veränderungen auf den Landoberflächen halb- oder vollautomatisch mit Hilfe von multitemporalen Fernerkundungsdatensätzen zu erfassen. Die Methoden können grob in zwei Kategorien aufgeteilt werden: Vor- und Nachklassifizierungsmethoden.

\subsection{Veränderungsnachweis mit Vorklassifizierung}

Als Vorklassifizierung kommen folgende Methoden häufig zur Anwendung:

Abbildungsalgorithmus: Bei dieser Methode werden die mathematischen Beziehungen zwischen zweien bzw. mehren Datenquellen hergestellt und analysiert. Zu dieser Kategorie gehören u.a. Methoden der Bilddifferenzierung, Ratiobildung oder Bildregression. Die Eingangsdaten sind Roh- bzw. transformierte oder normalisierte Daten z.B. verschiedene Vegetationsindizes oder Hauptkomponenten.

Multitemporale Komposite: Die multitemporalen Daten werden in einem Datensatz kombiniert benutzt. Aus zwei 6-kanaligen Landsat TM-Dateien werden z.B. eine neue 12-kanalige Datei hergestellt. Die neue Datei wird dann für die weiteren Analysen herangezogen. Zur Analyse gehören u.a. die direkte multitemporale Klassifikation oder die Analyse des Veränderungsvectors. 
Zur detaillierten Beschreibung der o.a. Verfahren wird insbesondere auf die Arbeiten von JENSEN (1996) und Singh (1989) verwiesen. Im folgenden wird die in dieser Untersuchung verwendete Nachklassifizierungsmethode bezüglich ihrer Vor- und Nachteile im Vergleich zur Vorklassifizierungsmethode diskutiert.

\subsection{Veränderungsnachweis mit Nachklassifizierung}

Bei der Nachklassifizierungsmethode wird eine vollständige Klassifizierung der jeweils einzelner der multitemporalen Daten benötigt. Die einzelnen klassifizierten Bilder werden dann pixelweise verglichen und eine Veränderungsmatrize hergestellt. Mit Hilfe der Veränderungsmatrize werden dann die Veränderungen in den einzelnen Klassen identifiziert und quantitativ analysiert. Mit der Vorklassifizierungsmethode werden zwar die veränderten Flächen identifiziert, aber nicht die Art der Veränderung erfaßt. Dies ist ein großer Vorteil der Nachklassifizierungsmethode gegenüber den Vorklassifizierungsmethoden.

Ein anderer Vorteil der Nachklassifizierungsmethode ist die nicht unbedingt erforderliche radiometrische Korrektur bzw. Normalisierung. Bei der Klassifikation der einzelnen Daten hat die atmosphärische Korrektur nur einen geringen Einfluss auf die Ergebnisse der Klassifikation (SoNG et al. 2001). Damit können die Probleme, die oben als negative Einflußfaktoren erwähnt wurden (Unterschiede in den atmosphärischen Bedingungen, der Bodenfeuchtigkeit, der Kalibrierung des Sensors und im phänologischen Zustand der Vegetation), minimiert werden.

Der Hauptnachteil der Nachklassifizierungsmethode ist die Fehlerfortpflanzung. Fehler, die bei der individuellen Klassifikation entstehen, pflanzen sich in den Ergebnissen des Veränderungsnachweises fort. So ergibt sich z.B. bei zwei Klassifikationen mit jeweils $80 \%$ Genauigkeit nur eine Genauigkeit von $64 \%$ bei einem Veränderungsnachweis.

Trotz der Nachteile wurde in einer vergleichenden Untersuchung von mehreren Veränderungsnachweismethoden gezeigt, dass die Methode der Nachklassifizierung mit einer überwachten Klassifizierung die höchsten Genauigkeiten erzielt (MAS 1999). Die Nachklassifizierungsmethode ist daher die am meisten verwendete Methode für quantitative Analysen bei Veränderungsnachweis-Untersuchungen. 


\section{Ergebnisse}

In diesem Kapital wird zunächst die Bereitstellung von Grundlagedaten für die Auswertung der Satellitendaten vorgestellt. Hierzu gehören die Herstellung eines digitalen Geländemodells (Abschnitt 5.1), die Orientierung der Luftbilder, die Kartierung der Landnutzungen, die Herstellung digitaler Orthophotos (Abschnitt 5.2), die Gewinnung von Paßpunkten für die Georeferenzierung der Landsat TM und der IKONOS Geo-Daten und die Gewinnung der Referenzdaten sowohl für die Klassifizierung als auch der Verifizierung der Landsat TM Satellitenaufzeichnugen. Anschießend werden im Abschnitt 5.3 die Ergebnisse der vorbereitenden Arbeiten für die Auswertung der Landsat TM und IKONOS Daten einschließlich der geometrischen Korrektur, der topographischen Normalisierung und der Fusion multispektraler Daten mit panchromatischen Daten zusammengefaßt.

Die Ergebnisse der pixel- und segmentbasierten Klassifikation und die Vergleichsstudien beider Methoden werden in Abschnitt 5.4 und 5.5 dargestellt. In dem letzten Abschnitt 5.6 wird die Erfassung der Waldveränderung erläutert.

\subsection{Herstellung des digitalen Geländemodells}

Ein digitales Geländemodel (DGM) und daraus abgeleitete Informationen wie Hangneigung und Exposition können wichtige Informationen sowohl für die Auswertung der Satellitendaten als auch für die Analyse der Ergebnisse liefern. In der vorliegenden Untersuchung wurde das DGM für die Orthorektifizierung und für die topographische Normalisierung der Landsat TM Daten eingesetzt. Für die Herstellung des digitalen Geländemodells wird zuerst ein TIN (Triangulated Irregular Network) Model mit Hilfe von Höhenlinien sowie von Höhenwerten einiger Berggipfel erstellt, welche aus der digitalen topographischen Karte (siehe Abschnitt 3.231) entnommen wurden. Danach wurden zwei DGM mit der Rastergröße von 30m bzw. $1 \mathrm{~m}$ aus dem TIN Model abgeleitet. Die ausgewählten Rastergrößen entsprechen dem Auflösungsvermögen der in vorliegende Untersuchung verwendeten Satellitendaten Landsat TM und IKONOS. Diese Arbeiten erfolgten mit dem Softwarepaket ARC/INFO. Die Höhenwerte des DGMs liegen zwischen 478 und 1251m mit einer mittleren Höhe von $732.3 \mathrm{~m}$ über dem Meeresspiegel. 
Wobei Abbildung 5.1 zeigt eine dreidimensionale Darstellung des Untersuchungsgebietes, eine IKONOS Komposition mit R (Kanal 4), G (Kanal 3), B (Kanal 1) dem DGM überlagert wurde.

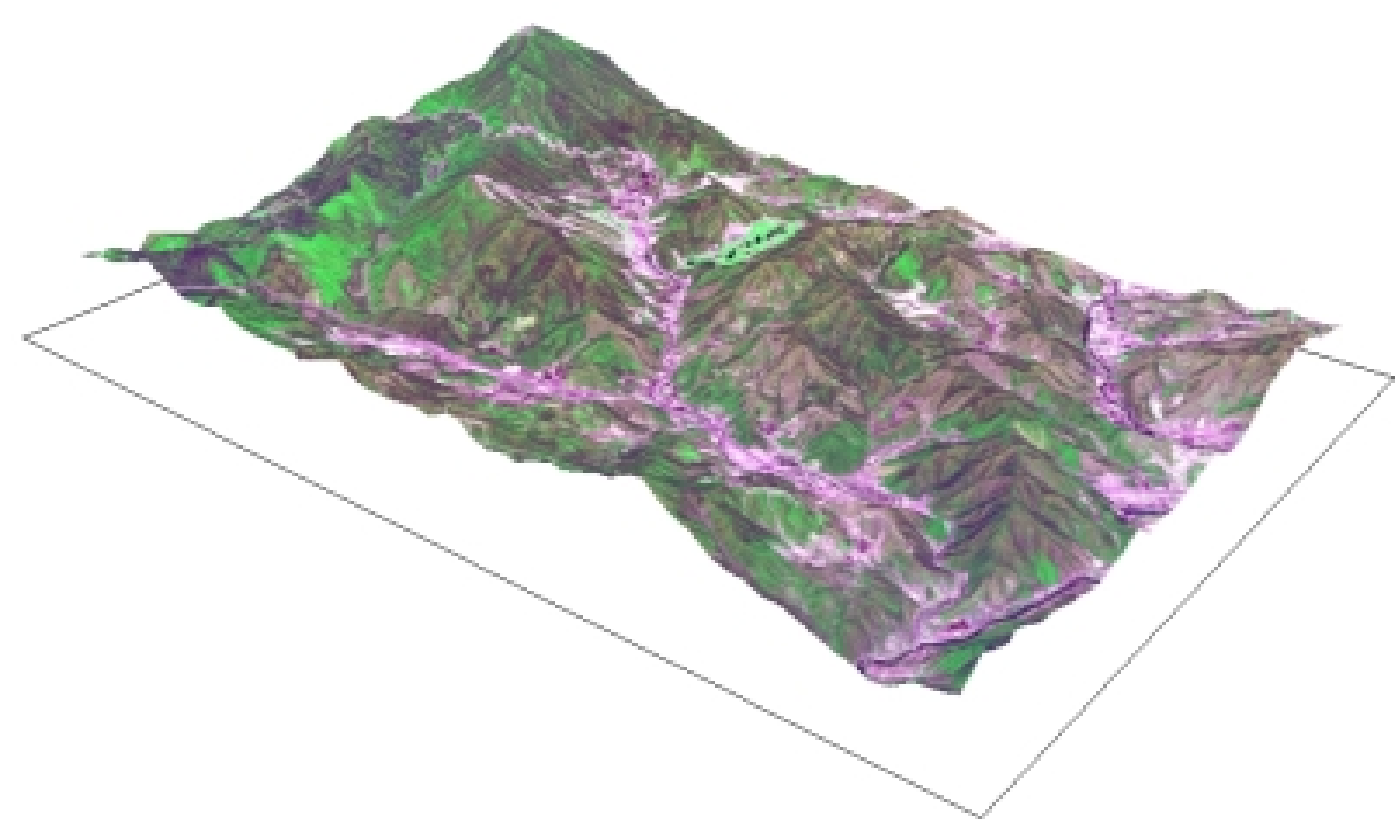

Abbildung 5.1: $\quad$ Dreidimensionale Darstellung des Untersuchungsgebietes.

\subsection{Auswertung der Luftbilder}

Aus rechtlichen Gründen war es nicht möglich, die Original-Luftbilder nach Deutschland mitzunehmen. Für die digitale photogrammetrische Auswertung der Luftbilder wurden die Bilder mit einem einfachen Büroscanner mit einer Auflösung von 300 dpi (ca. $85 \mu$ ), die bei einem mittleren Bildmaßstab von 1: 13000 ca. 1m auf dem Boden entspricht, digitalisiert und mit dem digitalen photogrammetrischen Auswertungssystem ,Softplotter“ ausgewertet.

\subsection{Aerotriangulation}

Da die Kamera-Kalibrierungsdaten für die innere Orientierung der Luftbilder nicht vorhanden waren, konnten hierfür nur Schätzwerte verwendet werden. Die Objektivverzerrungen wurden nicht berücksichtigt und der Bildmittelpunkt gleich dem Bildhauptpunkt gesetzt. Der Abstand der Bildrahmenmarken vom Bildhauptpunkt 
wurde nach mehren Messungen mit ca. 109,5 Millimeter ermittelt. Die Paßpunkte zur Aerotriangulation der Luftbilder wurden aus den digitalen und den analogen topographischen Karten im Maßstab 1:25000 vom „National Geography Institute (NGI)“ Koreas gewonnen. Insgesamt wurden 23 Paßpunkte, davon 6 Vollpaßpunkte, 12 Lagepaßpunkte und 5 Höhenpaßpunkte für die 30 Modelle überwiegend im Bereich von Straßenkreuzungen und Brücken gesammelt. Dabei wurden 107 Verknüpfungspunkte für die Aerotriangulation herangezogen. Die Abbildung 5.2 zeigt die Lage der verwendeten Paßpunkte. Damit konnte eine Lagegenauigkeit von 6,5 $\mathrm{m}$ in X-Richtung, 8,2 $\mathrm{m}$ in Y-Richtung und eine Höhengenauigkeit von 10,7 m erreicht werden.

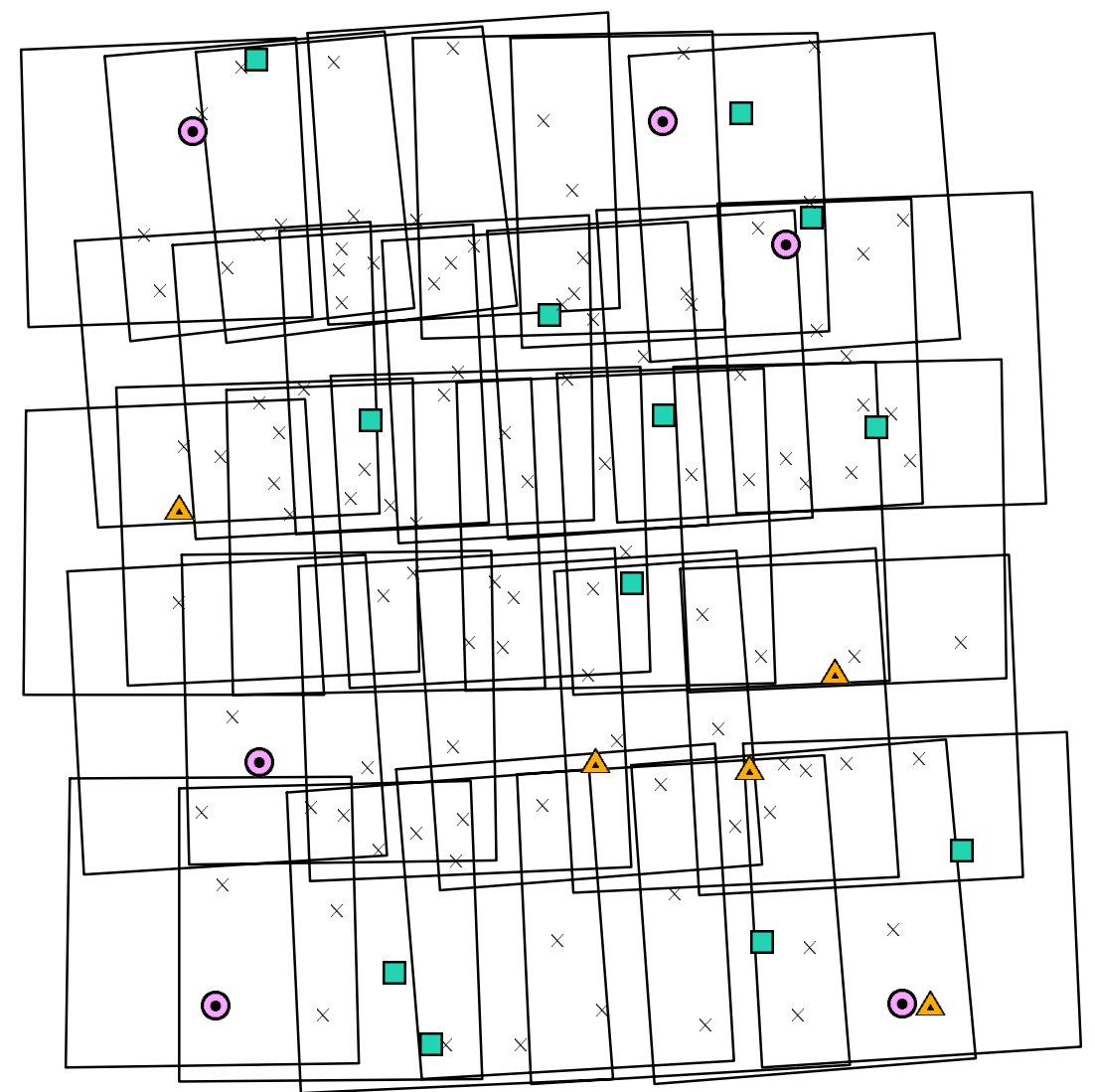

Abbildung 5.2: $\quad$ Verteilung der für die Aerotriangulation verwendeten Paßpunkte und Verknüpfungspunkte. Dabei bedeuten $\odot$ Vollpaßpunkte, $\square$ Lagepaßpunkte, $\triangle$ Höhepaßpunkte und $\times$ für die Verknüpfungspunkte. 


\subsection{Stereoauswertung der Luftbilder}

Nach der Aerotriangulation wurde ein Stereomodel für jedes Bildpaar erzeugt. Durch eine stereoskopische Interpretation wurden die „Ground Truth“ Daten für die Klassifikation der Landsat TM Daten und ihre Verifikation kartiert. Obwohl die bereits vorhandene Waldtypenkarte durch Interpretation der selben Luftbilder hergestellt wurde, war es nicht möglich diese Karte als Grundlage für „Ground Truth“ zu verwenden. Durch eine Überlagerung der vorhandenen Waldtypenkarte auf dem Stereomodel wurde festgestellt, daß die geometrische und thematische Genauigkeit der Waldtypenkarte für die Referenzdaten nicht geeignet ist. Dies konnte auch bei einer Überlagerung mit den IKONOS Daten bestätigt werden (Abbildung $5.3 \mathrm{a}, \mathrm{b}$ ).

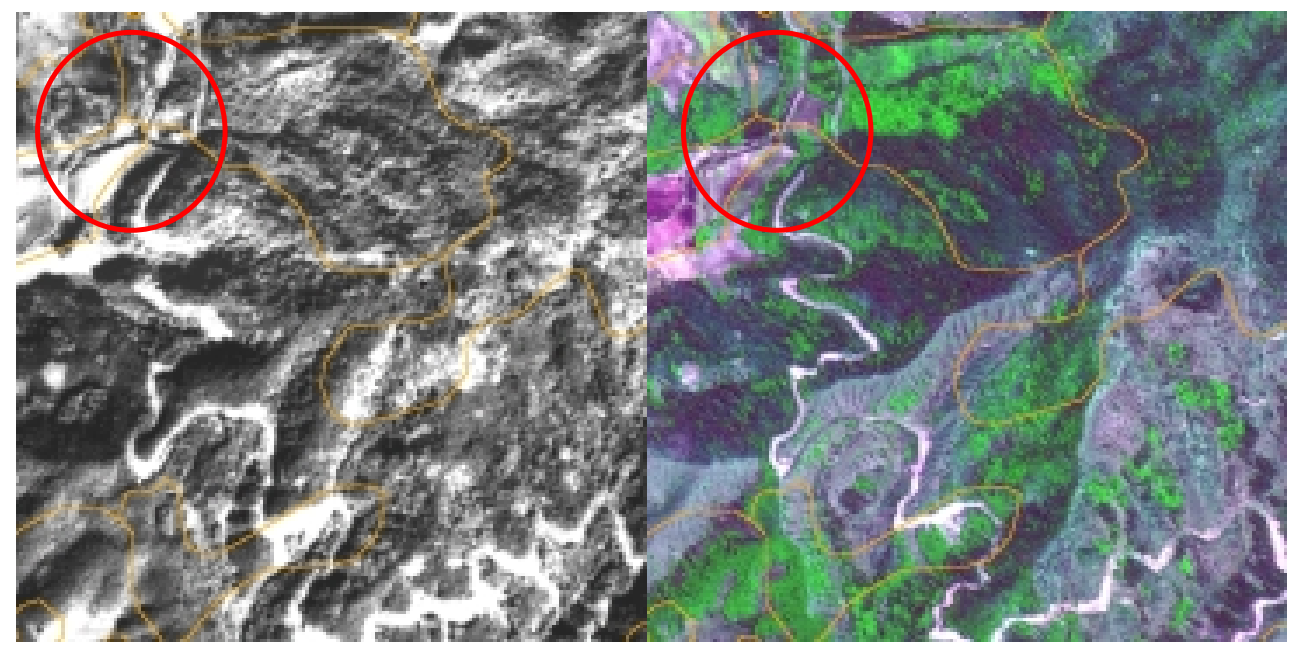

( a )

( b )

Abbildung 5.3: $\quad$ Überlagerung der vorhandenen Waldtypenkarte auf dem LuftbildOrthophoto (a) und den georeferenzierten IKONOS-Daten (b). Im Bereich des Kreises ist die geometrische Ungenauigkeit zu sehen.

Nach der Erstellung eines Klassifizierungsschemas (siehe Abschnitt 5.41) wurde die Lokalisierung und Kartierung der „Ground Truth“-Flächen anhand eines terrestrisch aufgenommenen Interpretationsschlüssels stereoskopisch am „Softplotter“ durchgeführt, wobei die IKONOS Daten und die vorhandene Waldtypenkarte als Hilfsmaterial zur Interpretation mit einbezogen wurden. 


\subsection{Orthophotoherstellung}

Ein digitales Orthophoto des Untersuchungsgebietes wurde mit dem Programmtool „Ortho Tool“ an dem digitalen photogrammetrischen System „Softplotter“ erstellt. Das Programmtool „Ortho Tool“ bietet die Erstellung eines Orthophotos mit einem DGModer TIN-Model. Obwohl aus dem Stereomodel ein DGM- bzw. TIN-Model erstellt werden kann, wurde in der vorliegenden Untersuchung aus zeitlichen Gründen das bereits aus den topographischen Karten hergestellte DGM herangezogen.

Für jedes orientierte Luftbild wird zunächst ein Orthophoto erstellt. Dabei wurde eine bilineare Transformation für die Zuweisung neuer Pixelwerte herangezogen. Anschließend wurden die einzelnen Orthophotos unter „ERDAS IMAGINE“ zu einem Orthophotomosaik zusammengesetzt. Auf eine radiometrische Korrektur vor dem Mosaikierungsprozeß wurde verzichtet, weil die verwendeten Luftbilder nicht so große Grauwertunterschiede innerhalb und zwischen den einzelnen Luftbildern aufwiesen und das Orthophotomosaik überwiegend zur Gewinnung der GCP („Ground Control Point“) dienen soll. In Abbildung 5.4 ist ein Überblick über das Orthophotomosaik und in Abbildung 5.5 ein kleines Teilgebiet des Orthophotos mit Höhenschichtlinien beispielhaft dargestellt.

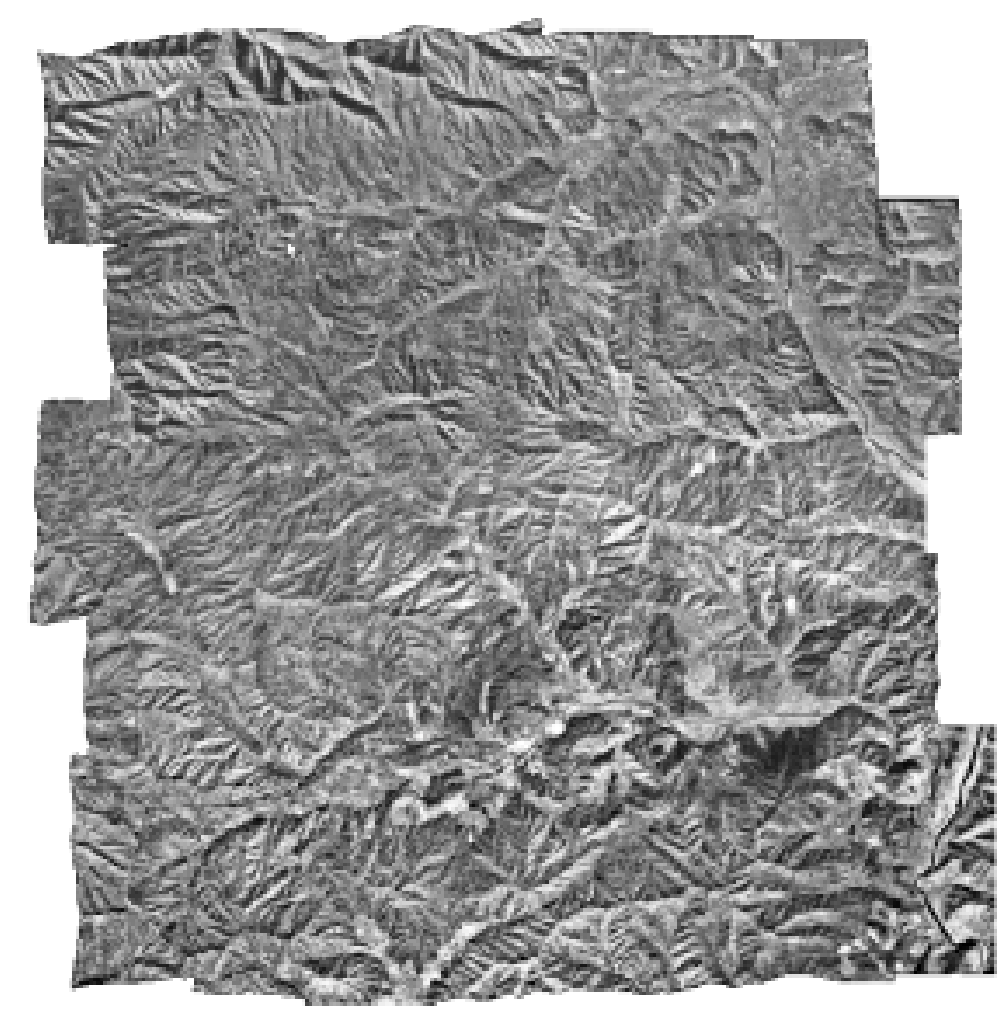

Abbildung 5.4: Orthophotomosaik aus 35 einzelnen Orthophotos 


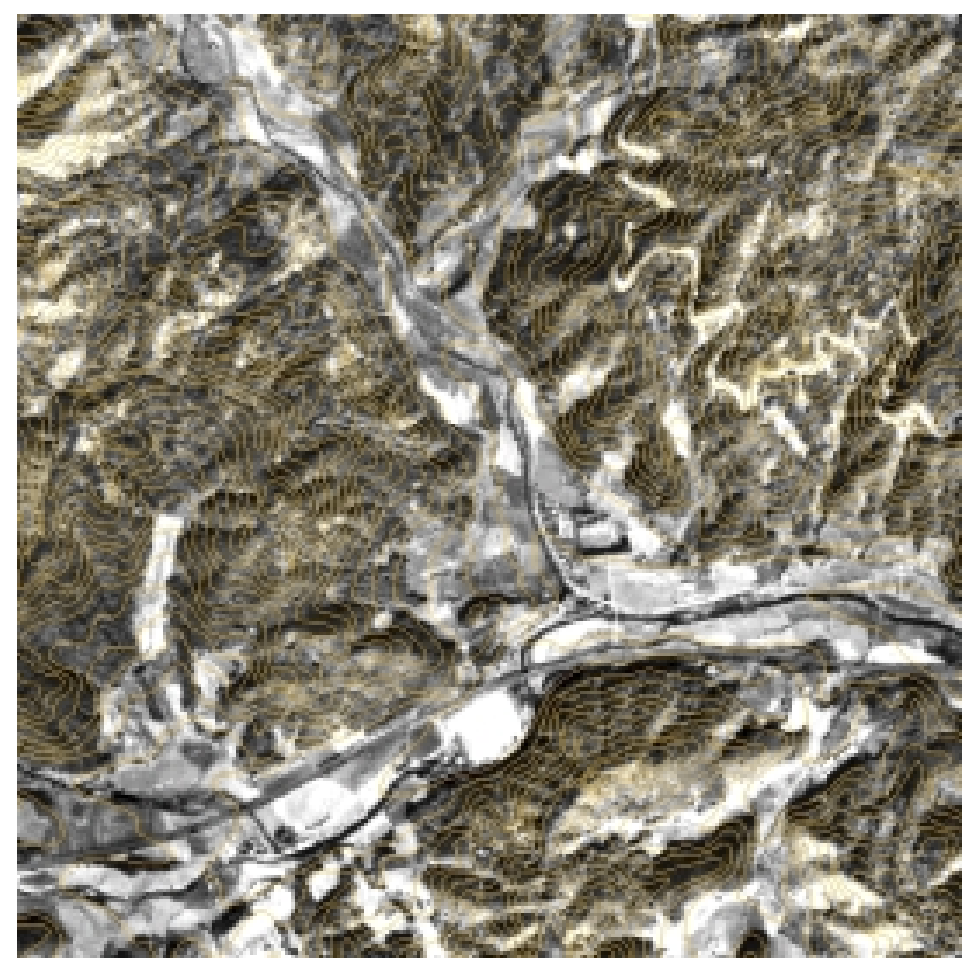

Abbildung 5.5: $\quad$ Darstellung des Orthophotos mit Höhenschichtlinien

\subsection{Vorverarbeitung der Satellitendaten}

Zur Vorverarbeitung einer Satellitenaufzeichnung gehören allgemein eine geometrische und radiometrische Korrektur und eine Bildverbesserungsprozedur. In dieser Arbeit wurde versucht, die multispektralen und panchromatischen Daten von IKONOS zu fusionieren und damit die Vorteile beider Datensätze gleichzeitig nutzbar zu machen (Abschnitt 5.31). In Abschnitt 5.32 werden die geometrischen Korrekturen im Rahmen der Georeferenzierung erläutert. Dabei liegen die Schwerpunkte auf der Erprobung verschiedener Georeferenzierungsmethoden und der Analyse ihrer Genauigkeit für die hochauflösenden Satelliten „IKONOS Geo“-Daten. Die Topographische Normalisierung der Landsat TM-Szene für die Eliminierung bzw. Minimierung topographiebedingter Einflüsse bei der Klassifizierung wird in Abschnitt 5.33 erläutert.

\subsection{Fusion der multispektralen und panchromatischen Kanäle des IKONOS Sensors}

Eine geometrisch genaue Registrierung der verschiedenen Datensätze ist eine unabdingbare Voraussetzung für die Fusion. Die IKONOS-Geo-Daten, die bereits in 
UTM Projektion geliefert wurden, sind nach Angaben von „Space Imaging (SI)“ mit einer Genauigkeit von 0,12 Pixeln bei einem LE90 (Linear Error 90\%) zwischen den multispektralen Kanälen miteinander registriert. Die geometrische Übereinstimmung zwischen den panchromatischen und multispektralen Kanälen beträgt 1,8 panchromatischen Pixel bei einem LE90. Daher konnten die beiden multispektralen und panchromatischen Datensätze ohne weiteres für die Fusion herangezogen werden.

Um eine unnötige Veränderung der Pixelwerte durch andere Bildverarbeitungen zu vermeiden wurde die Fusion der multispektralen Kanäle mit dem panchromatischen Kanal der IKONOS Daten als erster Schritt der Bildverarbeitung durchgeführt,.

\subsection{Fusion mit IHS Transformation}

Für die Fusion der IKONOS Daten wurden zunächst die originalen 11-bit Daten als 16bit Datenformat in „ERDAS IMAGINE“ importiert und die multispektralen Daten in einem 4-bandigen Datensatz zusammengesetzt. Bei einem farbbezogenen Fusionsverfahren wie die IHS Transformation können nur drei Kanäle vom RGB Farbsystem in einen IHS Farbsystem transformiert werden. Deswegen sollten zuerst drei aus der vier IKONOS Multispektralkanäle für das RGB Farbsystem ausgewählt werden. Zur Auswahl der optimalen Bandkombination mit minimalem Informationsverlust wurde eine Korrelationsanalyse durchgeführt. In Tabelle 5.1 sind die Korrelationskoeffizienten zwischen den vier multispektralen Kanälen der IKONOS Daten dargestellt. Wie Tabelle 5.1 veranschaulicht, zeigt sich die höchste Korrelation zwischen dem Kanal 1 (Blau) und 2 (Grün) mit einem Korrelationskoeffizienten von 0,98, wobei der Kanal 1 mit den anderen Kanälen eine geringe Korrelation zeigt. Aus diesem Grund wurde der Kanal 2 ausgelassen und die Kombination 3(Rot) 4(NahInfrarot) 1(Blau) für das RGB Farbsystem ausgewählt.

Tabelle 5.1: $\quad$ Korrelationskoeffizient zwischen den IKONOS Kanälen

\begin{tabular}{ccccc}
\hline Kanal & 1 & 2 & 3 & 4 \\
\hline 1 & 1 & 0,98 & 0,95 & 0,6 \\
2 & 0,98 & 1 & 0,97 & 0,67 \\
3 & 0,95 & 0,97 & 1 & 0,64 \\
4 & 0,6 & 0,67 & 0,64 & 1 \\
\hline
\end{tabular}


Mit der gewählten Bandkombination wurde zuerst die ursprüngliche geometrische Auflösung der multispektralen Kanäle von $4 \mathrm{~m}$ auf $1 \mathrm{~m}$ des panchromatischen Kanals verfeinert und dann ins IHS Farbsystem transformiert. Da die Fusion mittels IHS Transformation eine hohe Korrelation zwischen der Intensitätskomponente und den geometrisch hochauflösende panchromatischen Daten voraussetzt, wurde die Korrelation zwischen beiden Datensätzen durch Ermittelung des Korrelationskoeffizienten überprüft. Der Korrelationskoeffizient lag bei 0,91. Dies wurde auch durch eine visuelle Kontrolle bestätigt (siehe Abbildung 5.6). Nach der Transformation wurde die Intensitätskomponente mit den panchromatischen Daten ersetzt. Dabei wurde die Pixelwertverteilung des panchromatischen Datensatzes mit der Intensitätskomponente durch eine lineare Transformation angepaßt. Die Pixelwerte schwanken bei der Intensitätskomponente zwischen 0,75882 und 6,6627 und bei dem panchromatischen Datensatz zwischen 132 und 2047.

Schließlich wurde der ersetzte Datensatz wieder in das RGB Farbsystem rücktransformiert. Da es sich bei den rücktransformierten Datensatz um ein „Floatingpoint“ Datenformat mit ca. 1 GB Größe handelt, wurde es wieder auf 8-bit reduziert. So wurde ein fusioniertes Bild mit drei spektralen Kanälen und $1 \mathrm{~m}$ geometrischer Auflösung für die weiteren Arbeiten bereitgestellt. 


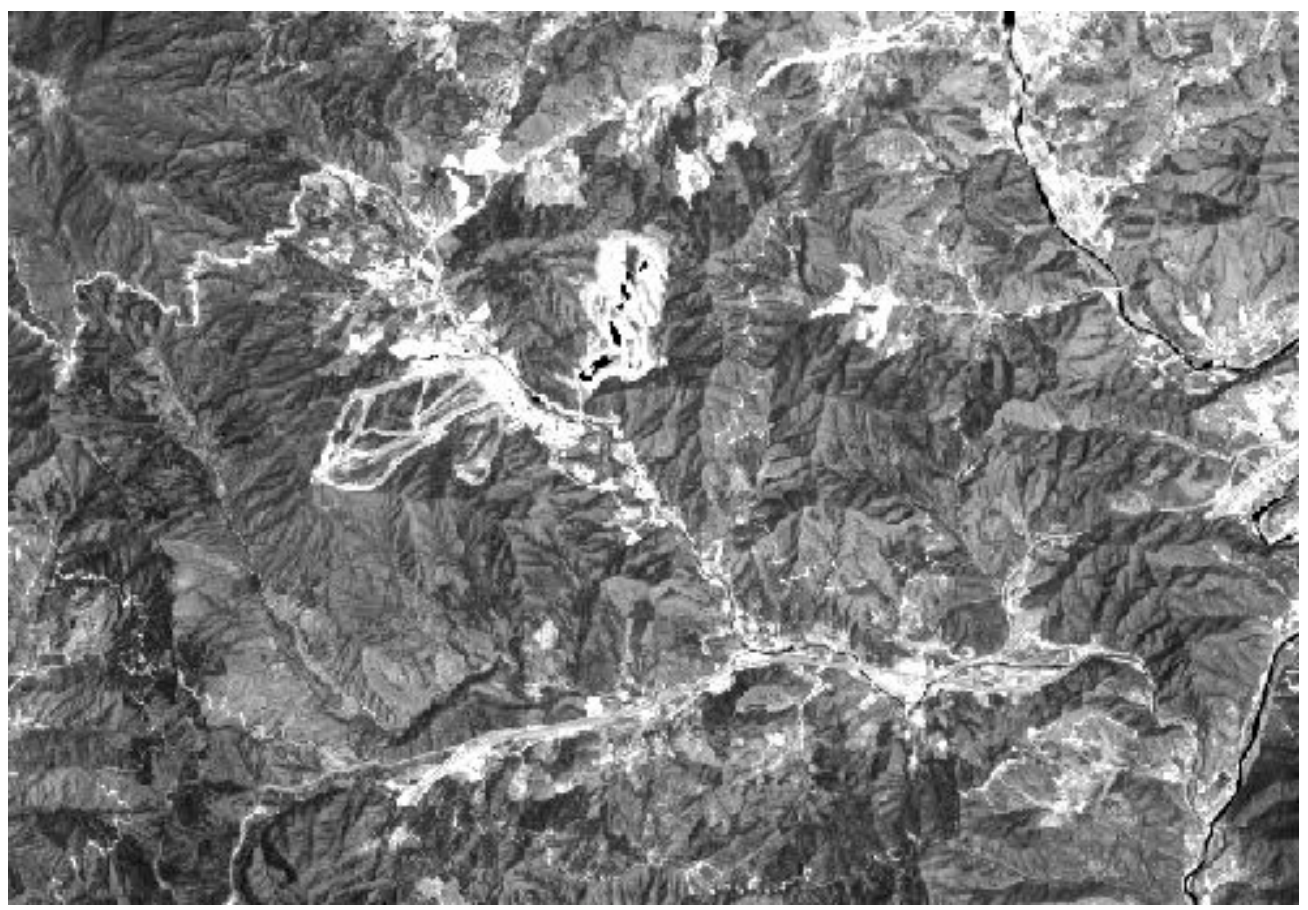

(a)

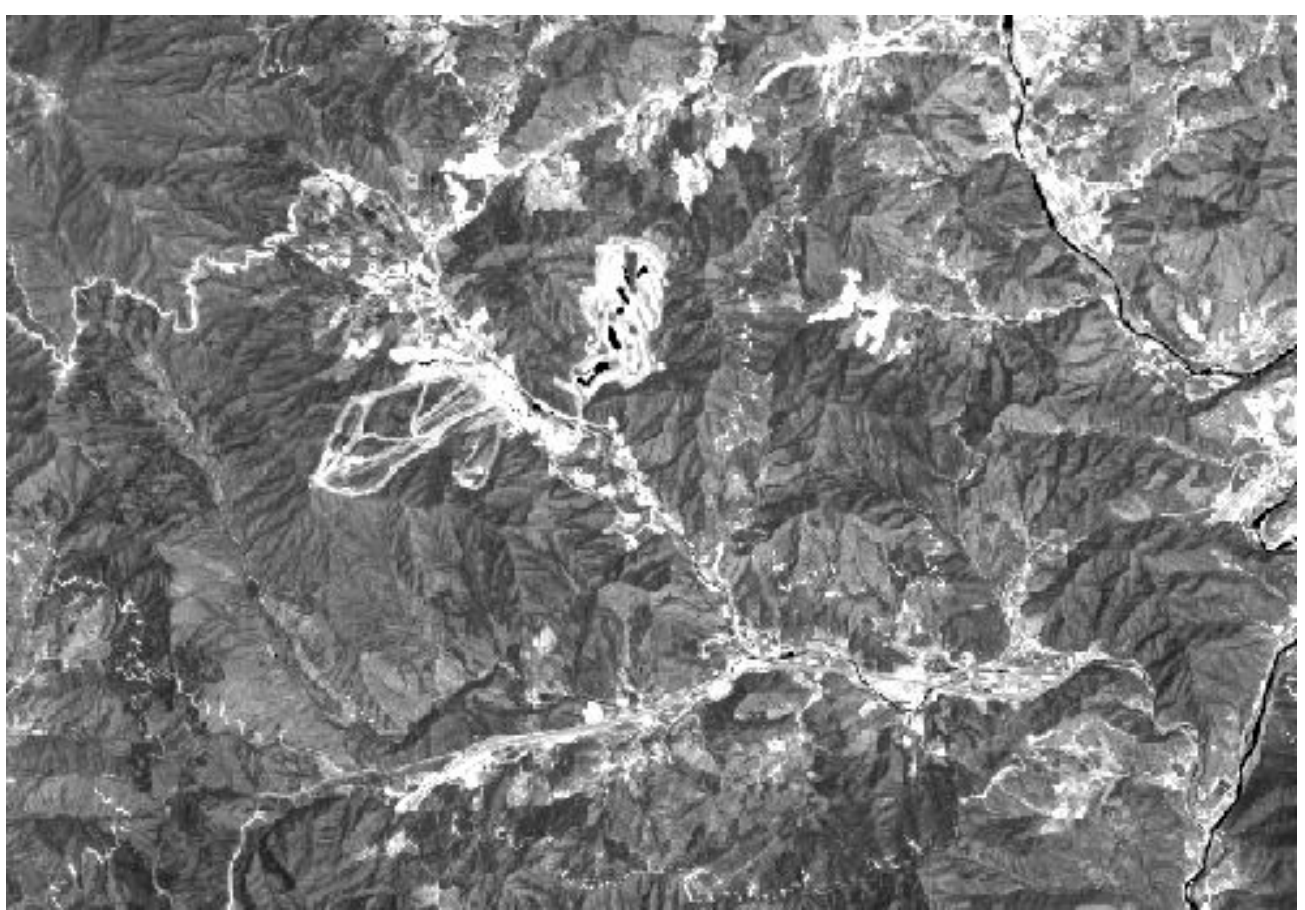

(b)

Abbildung 5.6: Vergleich der Intensitätskomponente in IHS Farbsystem (a) mit dem panchromatischen Datensatz (b). Die Pixelwertverteilung des panchromatischen Datensatzes wurde mit der Intensitätskomponente durch eine lineare Transformation angepaßt. 


\subsection{Qualität des fusionierten Bildes}

Durch visuelle Betrachtung wurde die Qualität des fusionierten Bildes evaluiert. Der Schwerpunkt lag dabei auf der Objekterkennbarkeit im Vergleich zum Bild der multispektralen Originalkanälen. Zur Beurteilung der Bildqualität vor und nach der Fusion wurde beispielhaft eine Autobahnausfahrt und eine Waldfläche mit neuer Pflanzung in Abbildung 5.7 gegenüber gestellt. Dabei zeigt sich, daß die fusionierten Bilder (rechts) wesentlich detaillierter als die originalen multispektralen Bilder (links) sind. Durch die hohe Auflösung sind z.B. sowohl die Fahrbahntrennlinie, als auch die Trennlinie zwischen den Ackerfeldern zu erkennen (Abb. 5.7 (a)). Eine hohe geometrische Auflösung ist ein wichtiges Kriterium nicht nur für die Kartierung sondern auch für die Identifizierung der Position von GCPs bei der Georeferenzierung. In Abbildung 5.7 (b) können sowohl die Pflanzreihen auf den neu gepflanzten Flächen, als auch die einzelnen Baumschatten in einem Altholzbestand erkannt werden.

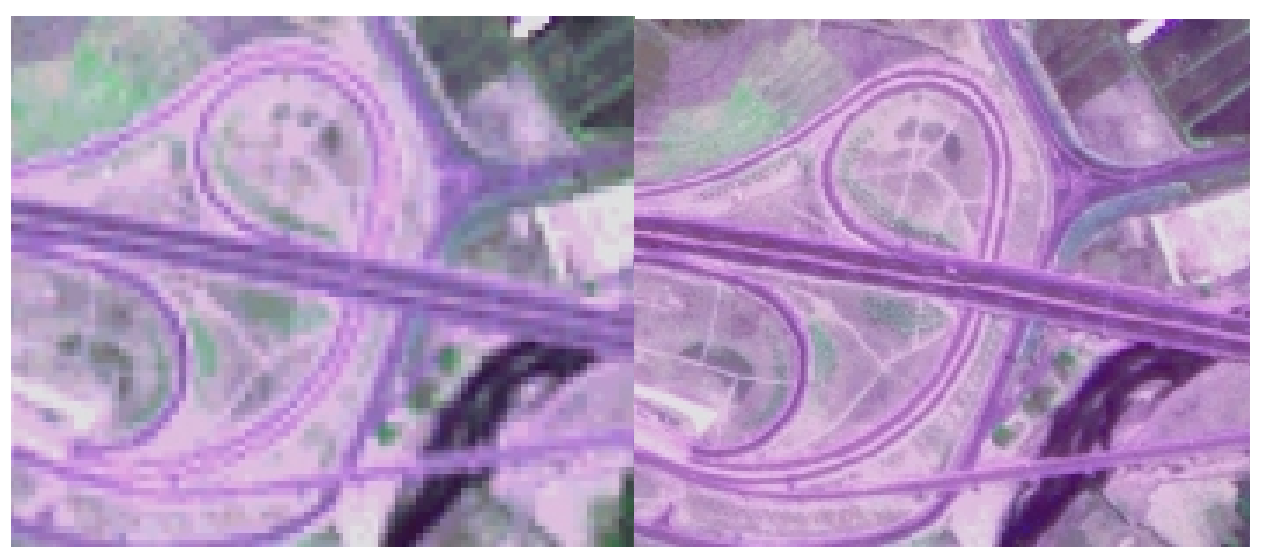

(a)

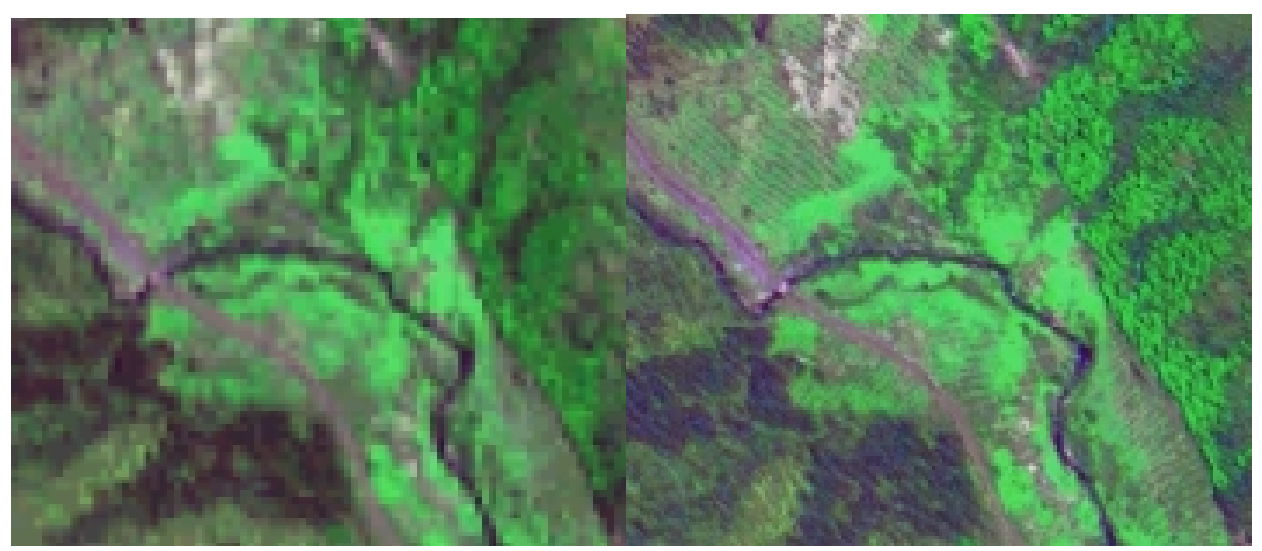

(b)

Abbildung 5.7: Gegenüberstellung der originalen multispektralen Bilder (links) und der fusionierten Bilder (rechts). 


\subsection{Geometrische Korrektur}

\subsection{Gewinnung von Paßpunkten}

Die Paßpunkte wurden mit Hilfe des mosaikierten Orthophotos gewonnen. Es wurden insgesamt 47 Punkte gemessen. Dabei wurde darauf geachtet, daß die Punkte im Bild gut verteilt sind und in den beiden Bildern, Luftbild-Orthophoto und fusioniertes IKONOS Bild, deutlich identifizierbar sind. Die Nutzung von Orthophotos bei der Paßpunktgewinnung lieferte gute Ergebnisse und gewährte eine große Erleichterung der Arbeit, besonders in einem Gebiet, welches überwiegend mit Wald bedeckt und wo dadurch die Gewinnung von Paßpunkten erschwert ist. Aus den gemessenen 47 Punkten wurden 27 Punkten zufällig ausgewählt und als GCP (Ground Controll Point) für die Georeferenzierung verwendet. Die restlichen 20 Punkte wurden dann als unabhängige Kontrollpunkte (ICP : Independent Controll Point) für die Überprüfung der Georeferenzierung herangezogen. In Abbildung 5.8 ist die Lage der verwendeten GCPs und ICPs graphisch dargestellt.

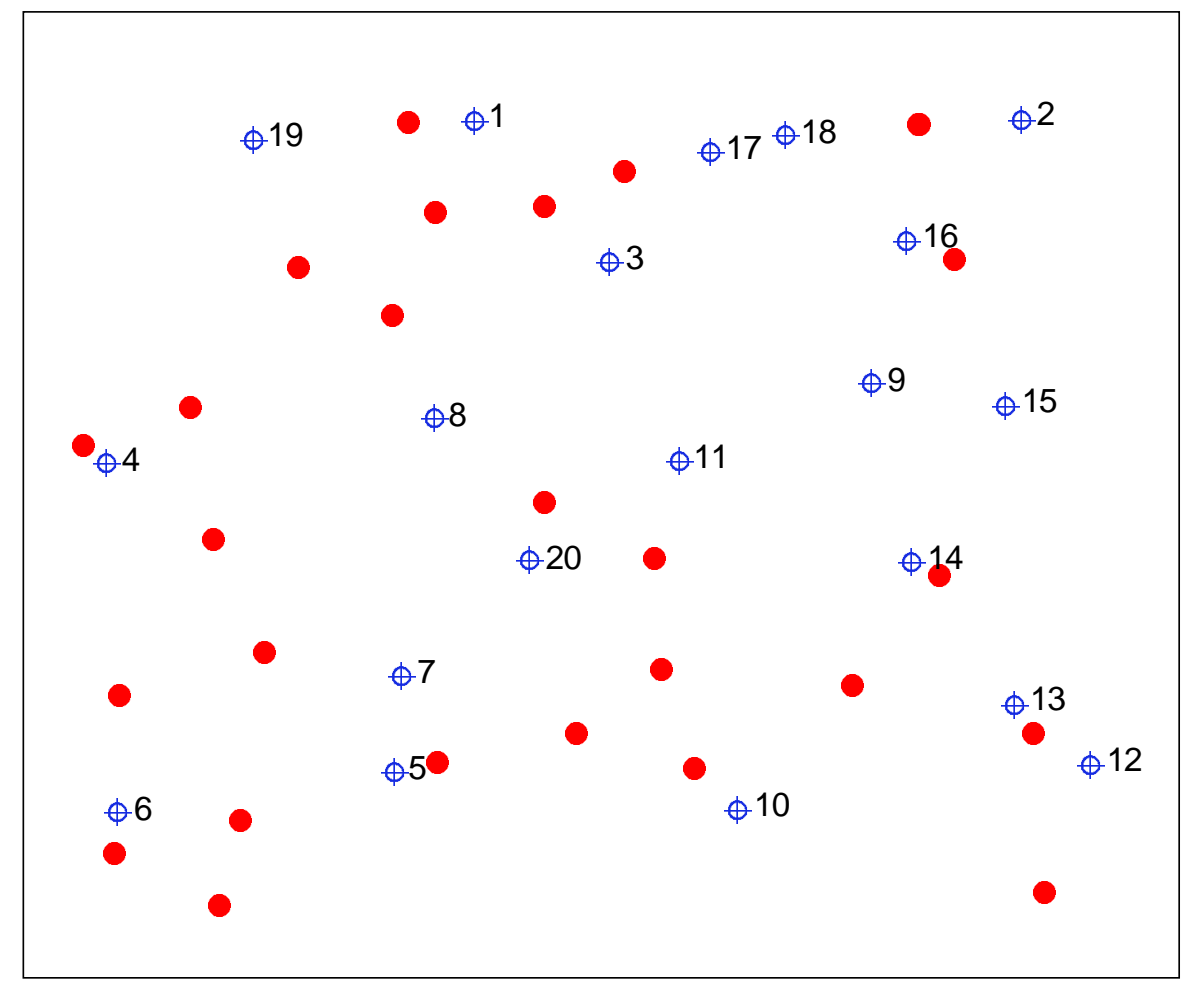

Abbildung 5.8: Verteilung der „Ground Control Points“ (GCP: ○) und der unabhängigen Kontrollpunkte (Independent Control Points: ICP : $\oplus$ ). 


\subsection{IKONOS}

Die in der vorliegenden Untersuchung verwendeten IKONOS Geo-Daten haben nach Angabe von ,Space Imaging (SI)“ einen Lagefehler von 50 m mit CE90 (Circular Error $90 \%$ ), d.h. alle Punkte im Bild liegen mit 90\% Wahrscheinlichkeit innerhalb einer Entfernung von nicht mehr als $50 \mathrm{~m}$ zu ihrer wahren Lageposition. Die Lagegenauigkeit der IKONOS Geo-Daten entspricht damit etwa den geometrischen Genauigkeitsanforderungen für eine Kartierung im Maßstab 1:100000.

Bei IKONOS Geo-Daten sind allerdings die reliefbedingten Verzerrungen bei den Angaben der Lagegenauigkeit nicht berücksichtigt. Deswegen sind die Lagefehler in reliefreichen Gebieten größer als die von „SI“ angegebenen Fehler. Außerdem wird die Lagegenauigkeit der Geo-Daten noch geringer, wenn die Szene im sog. „off-nadir“ Modus aufgenommen wird, was bei den IKONOS Daten der Normalfall ist. Die Lagefehler können in solch einem Fall bis zu mehren Hundert Meter betragen. Die in der vorliegende Untersuchung verwendeten IKONOS Geo Daten wurden nach Angaben der Metadaten mit einer „Nominal Collection Elevation“ 57,51640 Grad aufgenommen (Siehe Anhang 1).

Nachfolgend wird die Lagegenauigkeit der IKONOS-Geo-Daten überprüft und die Ergebnisse der geometrischen Korrektur mit einer Polynominal-Transformation und Orthorektifizierung dargestellt und ihre Lagegenauigkeit angegeben.

\subsubsection{Umprojektion}

Da die IKONOS Geo Daten bereits in eine Kartenprojektion entzerrt sind, konnten sie mit Hilfe des Bildverarbeitungsprogramms „ERDAS Imagine“ auf die in Korea verwendete Kartenprojektion „Transversale Mercator (TM)“ Projektion transformiert werden. Diese Methode benötigt keine Paßpunkte und ermöglicht eine schnelle und kostengünstige Georeferenzierung. In Tabelle 5.2 sind die Lagefehler des transformierten Bildes, berechnet nach verschiedenen Kriterien mit 20 ICPs (Independent Control Point) dargestellt. Die vollständigen Ergebnisse der einzelnen Kontrollpunkte sind in Anhang 2. (a) zu finden. In Abbildung 5.8 sind die Größe und Richtung der Fehler graphisch dargestellt. Die Lagefehler nach der Umprojektion können als die Lagegenauigkeit der IKONOS-Geo-Daten angesehen werden. 
Tabelle 5.2: $\quad$ Lagefehler der IKONOS Geo Daten nach der Umprojektion, berechnet anhand 20 unabhängiger Kontrollpunkte.

$(\mathrm{m})$

\begin{tabular}{cc}
\hline MDD & 60,7 \\
Std DD & 41,5 \\
CE 90 & 110,8 \\
RMSE X & 51,8 \\
RMSE Y & 51,3 \\
RMSE XY & 73,0 \\
\hline
\end{tabular}

MDD : Mean Difference Distance

Std DD : Standard Deviation of Difference Distance

CE 90 : Circular Error 90\%

RMSE : Root Mean Square Error

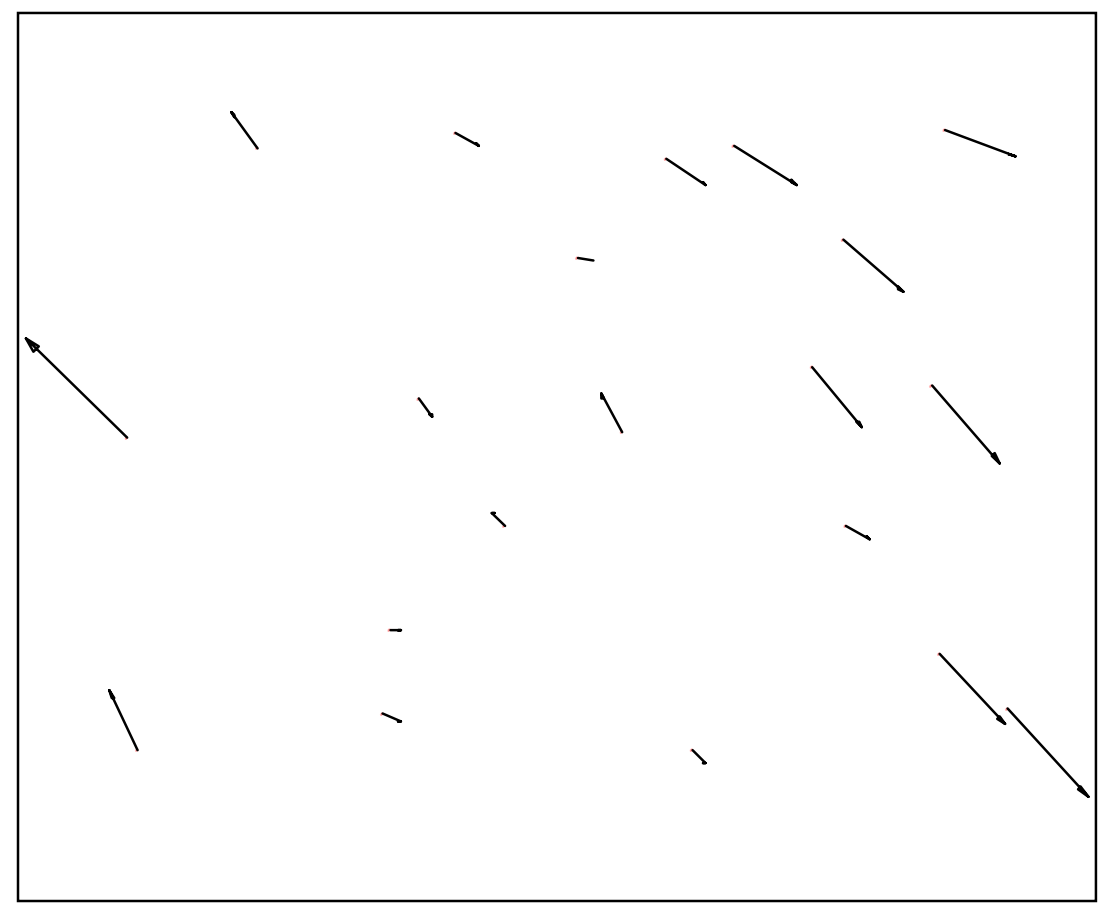

Abbildung 5.9: $\quad$ Graphische Darstellung der Lagefehler nach der Umprojektion. Die Fehlervektoren sind in 10-facher Vergrößerung dargestellt. Die Pfeile zeigen die Richtung des Fehlers an. Die Anfangspunkte der Pfeile zeigen die Position des unabhängigen Kontrollpunktes (Soll-Punkte) und die Endpunkte die entsprechende Position auf dem georeferenzierten Bild (IstPunkte) an.

Die Ergebnisse zeigen, daß der Lagefehler nach der Umprojektionwesentlich größer als der von „SI“ angegebene Lagefehler ist. Die maximale Lagefehler betragen bis zu 153 m während der mittlere Lagefehler 60,7 m ist. Der Fehler mit Angabe CE90 betrug 
110,8m und lag damit ebenfalls deutlich höher als der von „SI“ angegebene Fehler von 50m. Die Abbildung 5.9 zeigt, daß die Lagefehler räumlich unterschiedlich sind. Tendenziell sind die Lagefehler jedoch im linken und rechten Randbereich erheblich größer als im mittleren Bereich. Dies ist vermutlich sowohl auf die nicht vollständige Korrektur der „off-nadir“-Effekte bei der Aufnahme als auch auf die fehlende Berücksichtigung reliefbedingter Verzerrungen zurückzuführen.

\subsubsection{Polynominal Transformation}

Für die Auswahl des optimalen Polynomgrades wurden zunächst die RMS Fehler bei verschiedenen Polynomgraden mit 27 GCPs berechnet. In Tabelle 5.3 wurden die RMS Fehler in Abhängigkeit des Polynomgrades angegeben. Allgemein werden die RMS Fehler nach einer Erhöhung des Polynomgrades geringer, jedoch sind die RMS Fehler mit einem höherem als dem dritten Polynomgrad nur geringfügig verbessert. Deswegen wurde in der vorliegenden Untersuchung ein Polynom 3. Grades für die Transformation ausgewählt.

Tabelle 5.3: RMS Fehler nach einer Polynominal-Transformation mit unterschiedlichen Polynomgraden, ermittelt anhand von 27 GCP.

\begin{tabular}{cccccc}
\hline Polynom Grad & 1 & 2 & 3 & 4 & 5 \\
\hline RMSE x & 25.9 & 19.6 & 15.4 & 15.2 & 14.0 \\
RMSE y & 27.4 & 19.7 & 14.8 & 14.5 & 13.8 \\
RMSE xy & 37.7 & 27.8 & 21.4 & 21.0 & 19.7 \\
\hline
\end{tabular}

Zur Überprüfung der Georeferenzierung nach der Polynominal-Transformation wurde die Lagegenauigkeit mit Hilfe der 20 ICPs erneut berechnet (Tabelle 5.4, siehe auch Anhang 2.b). Der maximale und mittlere Lagefehler betrug dabei $99 \mathrm{~m}$ bzw. $31 \mathrm{~m}$. Obwohl die Lagefehler durch die polynominale Transformation kleiner geworden sind, ist der Fehler bei Angabe CE90 immer noch größer als der von „SI“ angegebenen Fehler von $50 \mathrm{~m}$. Der mit Hilfe von GCPs berechnete RMS Fehler war weniger als halb so groß wie derjenige, welcher mit Hilfe der ICPs ermittelt wurde. Dies zeigt, daß die Angabe der Lagegenauigkeit mit GCPs mit einer großen Unsicherheit behaftet ist. Deswegen sollte eine Überprüfung der Lagegenauigkeit in der Regel mit unabhängigen Kontrollpunkten durchgeführt werden. 
In Abbildung 5.10 wurden die Größe und Richtung der Lagefehler nach der polynominalen Transformation graphisch dargestellt. Im Vergleich zu den Ergebnissen nach der Umprojektion sind die Fehler im Randbereich durch die polynominale Transformation deutlich keiner geworden. Im Bereich der Bildmitte wurden die Lagefehler jedoch noch größer als vor der Transformation. Es konnte somit festgestellt werden, daß die Größe und Richtung der Lagefehler je nach der Position im Bild unterschiedlich ist.

Tabelle 5.4: Lagefehler der IKONOS Geo Daten nach der polynominalen Transformation, berechnet mit 20 unabhängigen Kontrollpunkten.

$(\mathrm{m})$

\begin{tabular}{cl}
\hline MDD & 31,1 \\
Std DD & 24,2 \\
CE 90 & 59,2 \\
RMSE X & 22,4 \\
RMSE Y & 32,0 \\
RMSE XY & 39,0 \\
\hline
\end{tabular}

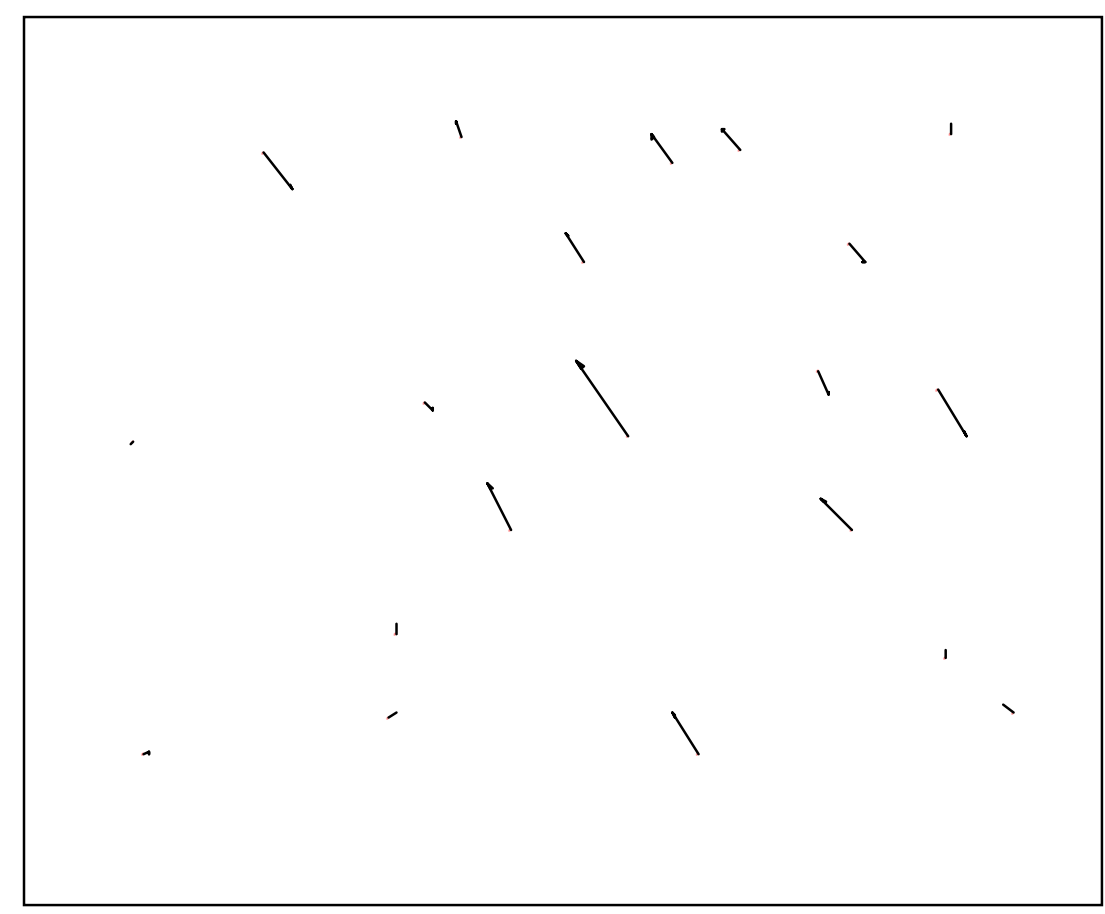

Abbildung 5.10: Graphische Darstellung der RMS Fehler nach der Polynominal Transformation. Zur Beschreibung der Abbildung siehe Abbildung 5.9. 


\subsubsection{Orthorektifizierung}

Die Orthorektifizierung der IKONOS-Geo-Daten wurde mit Hilfe von „OrthoEngine“ in dem Bildverarbeitungssystem „PCI“ durchgeführt. Da in der vorliegende Untersuchung die fusionierten Daten als Eingangsdaten zur Orthorektifizierung einbezogen wurden und die „OrthoEngine“ direkt aus den Metadaten die Sensorinformationen ließt, mußten zuerst diese Metadaten für die fusionierten Daten vorbereitet werden. Als Metadaten für die fusionierten Daten wurden die umbenannten Metadaten für die panchromatischen Daten verwendet, weil beide Daten die gleichen geometrischen Charakteristiken besitzten. Das im Abschnitt 5.1 beschriebene DGM mit 1 m Rastergröße wurde für die Prozedur mit einbezogen.

Die gleichen GCPs der Polynomtransformation wurden auch für die Orthorektifizierung verwendet. Tabelle 5.5 gibt den bei der Orthorektifizierung ermittelten RMS Fehler mit 27 GCPs an und zeigt deutlich verbesserte Ergebnisse im Vergleich zu den Ergebnissen der Polynomtransformation.

Tabelle 5.5: $\quad$ RMS Fehler bei der Orthorektifizierung anhand von 27 GCPs.

\begin{tabular}{lcc} 
& & $(\mathrm{m})$ \\
\hline RMSE $x$ & 2.5 \\
RMSE y & 2.2 \\
RMSE xy & 3.3 \\
\hline
\end{tabular}

In Tabelle 5.6 sind die Lagefehler nach der Orthorektifizierung mit unabhängigen Kontrollpunkten angegeben. In Anhang 2 (c) wurden die vollständigen Ergebnisse der einzelnen Kontrollpunkte widergegeben. Der mittlere Lagefehler lag bei 9,5 m und die maximale Abweichung bei 24,6 m. Der Lagefehler von 16,8 m bei CE90 liegt zwischen der Genauigkeit der von „SI“ definierten Kategorien „Reference“ und „Pro“, die jedoch ca. 3- bzw. 4-fach teuer als Daten der Genauigkeitskategorie „Geo“ sind.

Die RMS Fehler (7,9 $\mathrm{m}$ in X- und 7,7 $\mathrm{m}$ in Y-Richtung) liegen sehr nahe an den RMS Fehlern bei der Luftbildaerotriangulation (6,5 und 8,2 $\mathrm{m}$ in X- und Y-Richtung). Obwohl ein direkter Vergleich der Genauigkeit mit beiden Ergebnissen nicht möglich ist, kann dieser Verglich einen Anhaltspunkt für die Interpretation der Ergebnisse 
geben, weil die Genauigkeit der Orthophotos, aus denen die Lagekoordinaten der GCPs und ICPs extrahiert wurden, die Orthorektifizierung beeinflußt.

In Abbildung 5.11 kann festgestellt werden, daß die Orthorektifizierung relativ einheitliche Lagefehler über das gesamte Bild aufweist.

Tabelle 5.6: Lagefehler der IKONOS-Geo-Daten nach der Orthorektifizierung, berechnet mit 20 unabhängigen Kontrollpunkten.

(m)

\begin{tabular}{cc}
\hline MDD & 9,5 \\
Std DD & 5,8 \\
CE 90 & 16,8 \\
RMSE X & 7,9 \\
RMSE Y & 7,8 \\
RMSE XY & 11,1 \\
\hline
\end{tabular}

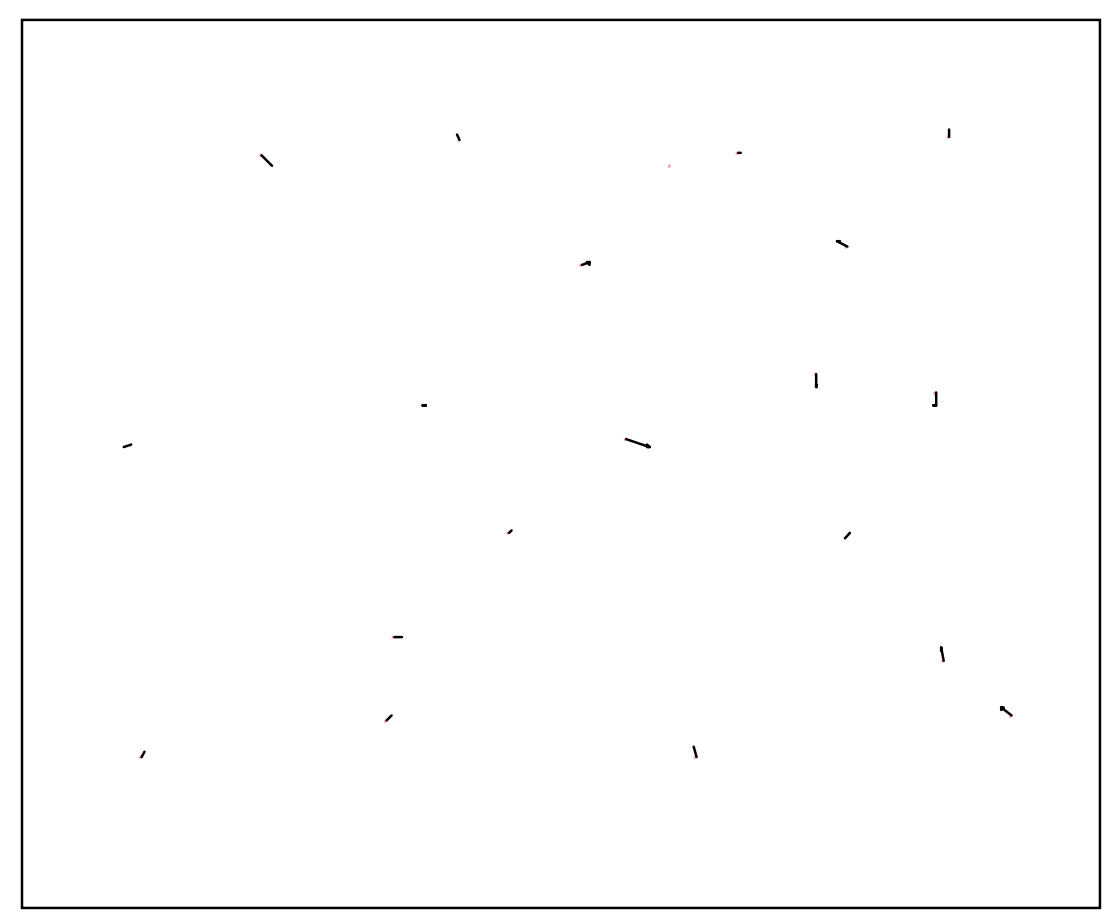

Abbildung 5.11: Graphische Darstellung der RMS Fehler nach der

Orthorektifizierung. Zur Beschreibung der Abbildung siehe Abbildung 5.9. 


\subsection{Landsat TM}

Die Identifizierung der GCPs in den Landsat TM Daten war wegen ihrer groben Auflösung relativ schwer. Es konnten nur 23 Punkte aus den im Orthophoto bestimmten GCPs im Bild identifiziert worden. Weil mindestens 20 unabhängige Kontrollpunkte (ICPs) für die Überprüfung der Lagegenauigkeit empfohlen werden (FDGC, 1998), würden dann eine nicht genügende Anzahl von Punkten als GCPs verbleiben. Daher wurde auf eine Überprüfung der Lagegenauigkeit mit unabhängigen Kontrollpunkten verzichtet. Die Transformation wurde anhand von 23 GCPs durchgeführt.

Die geometrische Korrektur der Landsat TM wurde mit einer Polynomtransformation zweiten Grades und mit einem Orthorektifizierungsverfahren durchgeführt. Polynome zweiten Grades wurden bereits in zahlreiche Untersuchungen angewendet und deren Eignung bereits mehrfach nachgewiesen. Die Orthorektifizierung wurde mit Hilfe eines DGMs mit einer 30 m Rastergröße vorgenommen. Die Georektifizierung der Landsat TM Daten wurde im Bildverarbeitungssystem „ERDAS Imagine“ durchgeführt.

In Tabelle 5.7 wurden die RMS Fehler bei der Transformation angegeben. Bei beiden Methoden zeigt sich fast der gleiche Fehler von etwa einem halben Pixel, was ca. $15 \mathrm{~m}$ auf dem Boden entspricht. Jedoch ist hier zu beachten, daß die RMS Fehler bei der Transformation nicht dem Fehler, der anhand von unabhängigen Kontrollpunkten ermittelt wird, entspricht.

Tabelle 5.7: $\quad$ RMS Fehler bei der Transformation

\begin{tabular}{ccc} 
& & (Pixel) \\
\hline RMS x & Polynom 2.-Grades & Orthorektifizierung \\
\hline RMS y & 0.5 & 0.5 \\
RMS xy & 0.5 & 0.4 \\
\hline
\end{tabular}

\subsection{Fazit der geometrischen Korrektur}

Bei hochauflösenden Satellitendaten wie IKONOS sind die Erwartungen an die Genauigkeit entsprechend höher. Diese Erwartungen gelten nicht nur für die thematische sondern auch für die geometrische Genauigkeit. Da die IKONOS-GeoDaten ohne Berücksichtigung der reliefbedingten Verzerrung georeferenziert wurden, waren die Lagefehler deutlich größer als die von „SI“ angegebenen Fehler. Obwohl 
durch eine polynominale Transformation die Lagefehler reduziert werden konnten, waren sie immer noch $\mathrm{zu}$ groß und räumlich unterschiedlich. Daher war eine Orthorektifizierung notwendig. Die Orthorektifizierung führte zu deutlich verbesserten Ergebnissen. Die Lagefehler waren kleiner und einheitlicher als bei den anderen verwendeten Methoden.

Bei den Landsat TM-Daten ergab sich kein großer Unterschied zwischen einer polynominalen Transformation und einer Orthorektifizierung. Trotzdem zeigt die Orthorektifizierung etwas bessere Resultate und wurde deswegen im weiteren Verlauf der Auswertungen verwendet. Als Vergleich der Ergebnisse wurde beispielhaft ein Ausschnitt der orthorektifizierten Satellitenbilder und des Luftbildorthophotos in Abbildung 5.12 dargestellt.

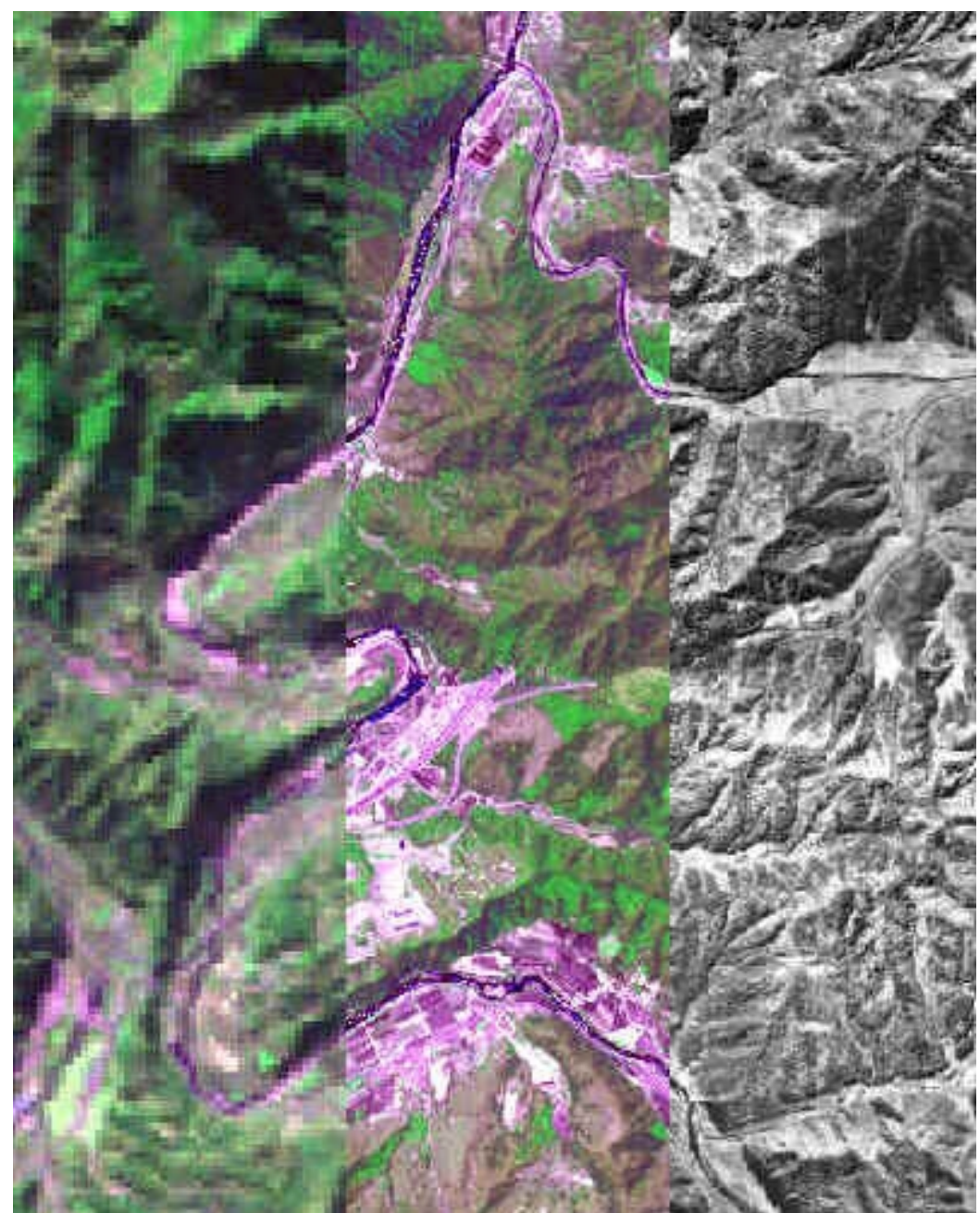

(a)

(b)

(c)

Abbildung 5.12: Vergleich des orthorektifizierten Satellitenbildes Landsat TM (a), der fusionierten IKONOS Szene (b) und des Luftbild-Orthophotos (c). 


\subsection{Topographische Normalisierung der Landsat TM}

Neben den Einflußfaktoren der topographischen Effekte, die bereits in Abschnitt 4.22 erläutert wurden, spielt die Sonnenhöhe eine große Rolle bei der Schattenbildung. Die Ausprägung der Schatten auf den IKONOS Daten war deutlich geringer als auf den Landsat TM-Daten, weil die Sonne zum Zeitpunkt der Aufnahme deutlich höher stand. Die Sonnenhöhe war bei der IKONOS Aufnahme 57,1 Grad und bei der Landsat TM Aufnahme nur 32,12 Grad (siehe Tabelle 3.4, 3.5 und auch Anhang 1). Die Unterschiede der Schattenprägung auf den beiden Bildern sind auch in Abbildung 5.12 und in Abbildung 5.14 (a) zu erkennen. Aufgrund der zum Teil tief eingeschnittenen Täler und großen Hangneigungen war eine topographische Normalisierung der TMSzenen dringend erforderlich. Auf eine topographische Normalisierung für die IKONOS Daten wurden in der vorliegende Untersuchung verzichtet, weil die reliefbedingten Grauwertunterschiede auf den IKONOS Daten nicht so groß wie bei den Landsat TM-Daten waren. Eine Normalisierung des Reliefeinflusses mit der Methode der Minnaertkorrektur wurde somit nur auf den Landsat TM Daten, welche ausgeprägte Reliefeffekte besitzt, vorgenommen.

Für die Berechnung der Minnaert-Konstanten soll normalerweise für jede einzelne Klasse eine eigene Minnaert-Konstante getrennt für jeden Spektralkanal abgeleitet werden, weil der Methode kein Lambertschen-Strahlungsmodel zugrunde liegt. Falls die topographische Normalisierung zum Zwecke einer besseren Klassifikation dienen soll, ist es nicht möglich, für jede einzelne Klasse eine eigene Minnaert-Konstante herzuleiten, weil die Klasse noch nicht bekannt ist. In der vorliegenden Untersuchung wurde deswegen nur eine Minnaert-Konstante für das gesamte Bild abgeleitet. Diese Methode wurde durch eine Voruntersuchung in ihrer Anwendbarkeit bereits bestätigt (CHO et al. 1999). Zur Berechnung der Minnaert-Konstante wurden zunächst mit Hilfe der Bildverarbeitungssoftware „ERDAS Imagine“ die Illumination $(\cos (i))$ und der Kosinus des Sonnenzenits $(\cos (\theta))$ des Untersuchungsgebietes berechnet. Dabei wurde das DGM mit $30 \mathrm{~m}$ Rastergröße mit herangezogen. Durch eine logarithmische Regression wurde die Minnaert-Konstante für jeden spektralen Kanal hergeleitet. Abbildung 5.13 stellt graphisch die Berechnung einer Minnaert-Konstanten beispielhaft dar und in Tabelle 5.8 wurden die Ergebnisse der Minnaert-Konstanten für die einzelnen Kanäle angegeben. 


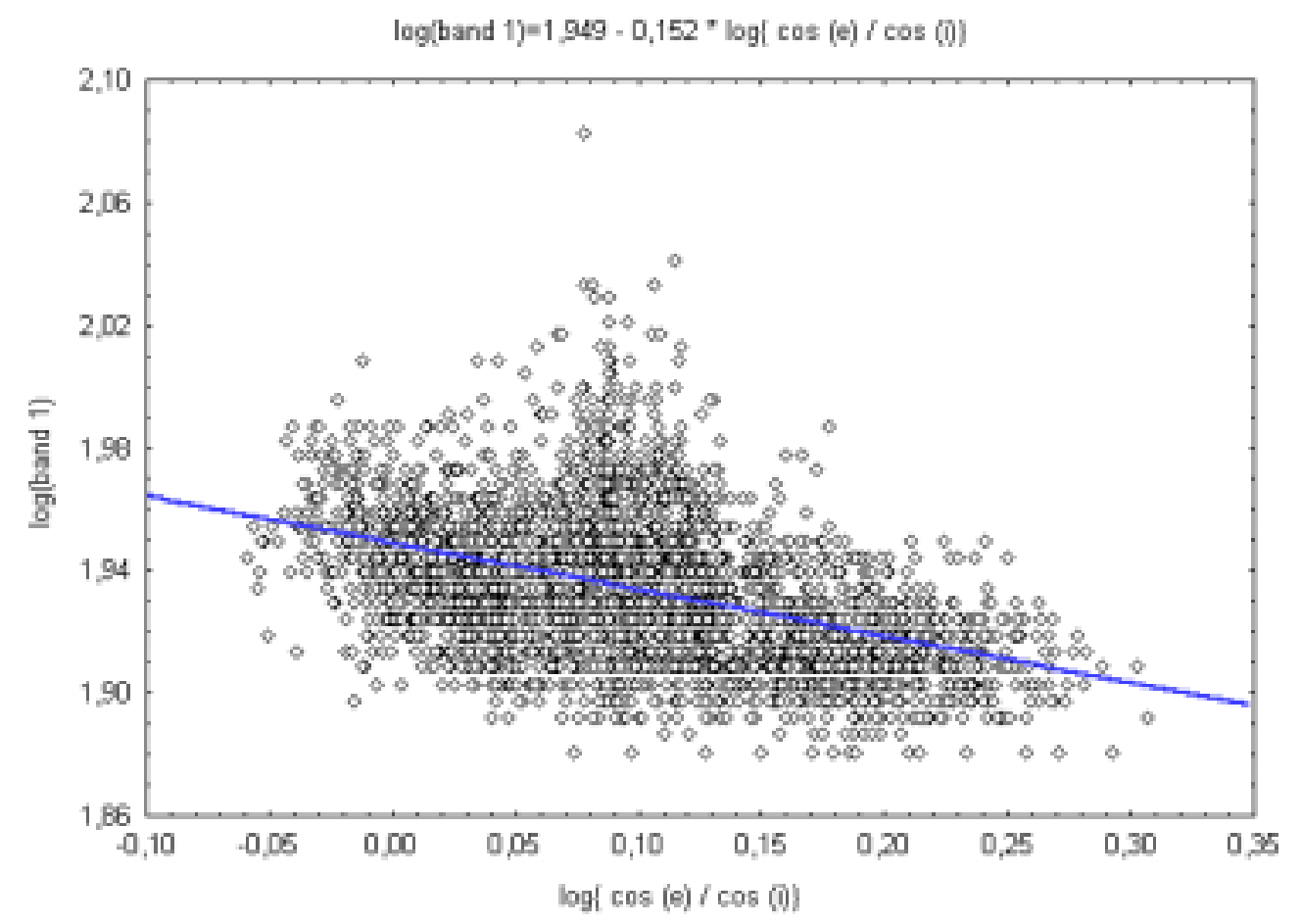

Abbildung 5.13: Berechnung der Minnaert-Konstanten. Die Steigung der

Regressionslinie ist mit umgekehrtem Vorzeichen die Minnaert Konstante.

Tabelle 5.8: $\quad$ Ergebnisse der berechneten Minnaert-Konstanten.

\begin{tabular}{c|cccccc}
\hline Band & 1 & 2 & 3 & 4 & 5 & 7 \\
\hline $\begin{array}{c}\text { Minnaert } \\
\text { konstante }\end{array}$ & 0,152 & 0,170 & 0,2770 & 0,467 & 0,705 & 0,740 \\
\hline
\end{tabular}

Mit den berechneten Minnaert-Konstanten wurde die topographische Normalisierung durchgeführt. Abbildung 5.14 zeigt den Vergleich zwischen den originalen Bilddaten (a) und den Ergebnissen der topographischen Normalisierung (b). Durch visuelle Betrachtung konnte festgestellt werden, daß die topographiebedingten Beleuchtungsunterschiede in der originalen Szene klar ersichtlich sind. Dagegen konnten die reliefbedingten Beleuchtungsunterschiede in der korrigierten Szene weitgehend normalisiert werden.

Die Auswirkung der topographischen Normalisierung auf die Klassifikation wurde durch verbesserte Klassifikationsergebnisse in einer anderen Untersuchung bereits nachgewiesen (CHO et al. 1999). 


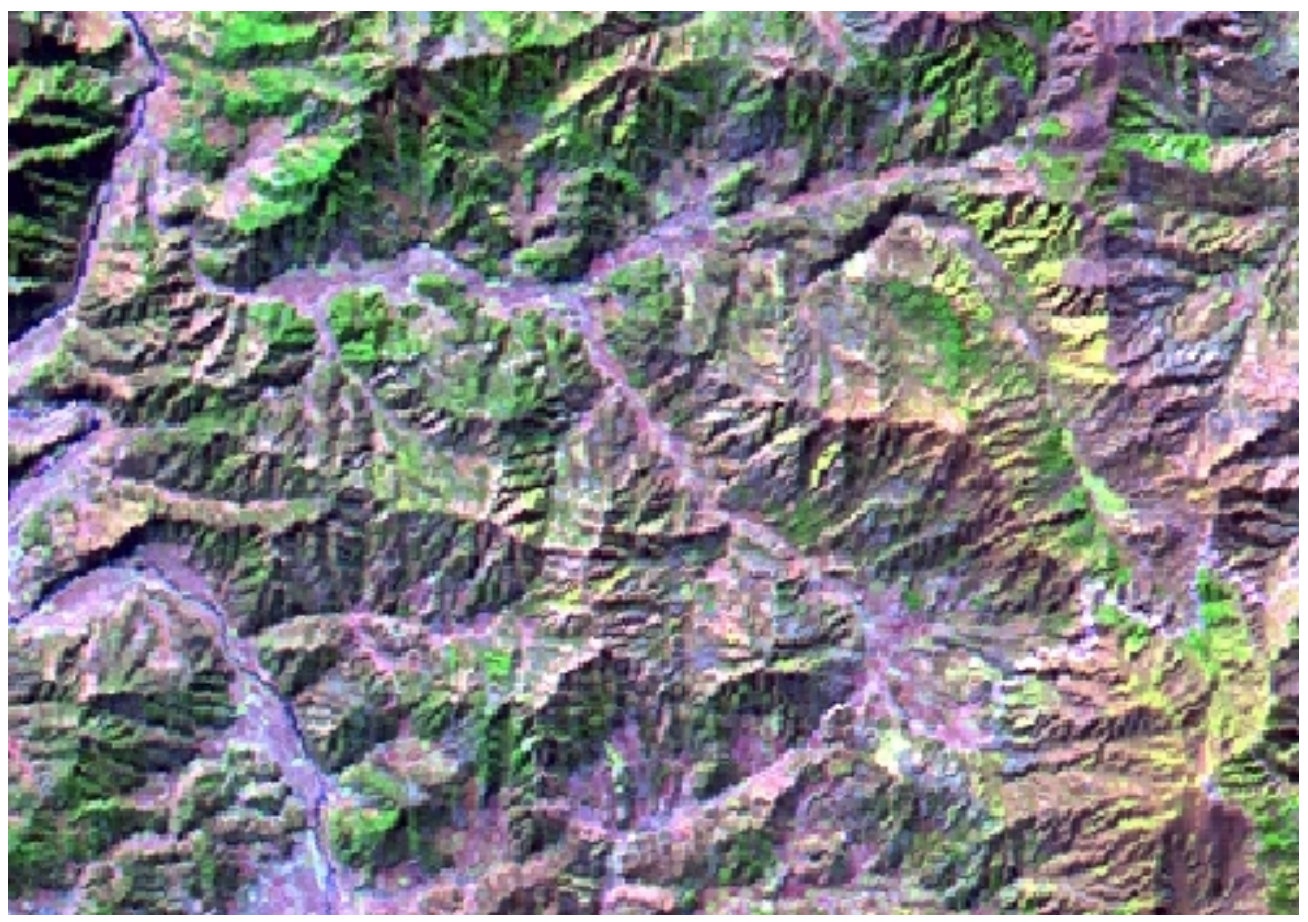

(a)

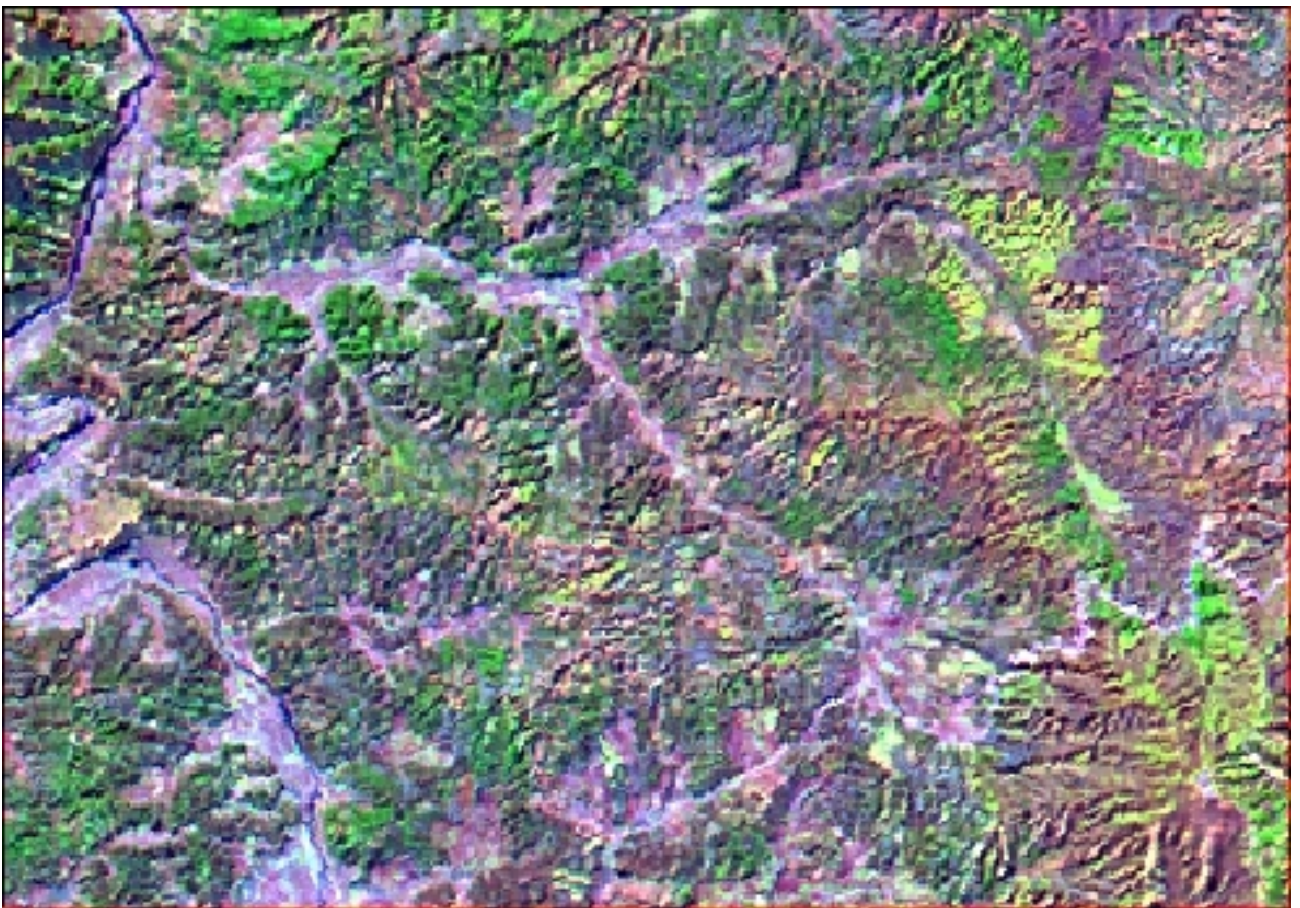

(b)

Abbildung 5.14: Vergleich zwischen den Originaldaten (a) und den

Korrekturergebnissen (b) nach der Minnaert-Korrektur für die Landsat TM-

Daten. Dargestellt wurde die Kanalkombination 5, 4, 3 für die RGB

Farbkanäle. 


\subsection{Klassifikation der Satellitendaten}

Die Klassifikation der Satellitendaten wurde sowohl durch die herkömmliche pixelbasierte Methode als auch durch die segmentbasierte Methode durchgeführt. Für die pixelbasierte Klassifikation wurde die „Maximum Likelihood“-Methode herangezogen. Als Segmentbasierte Klassifikation wurden sowohl die Methode der Mehrheitszuweisung nach einer pixelbasierten „Maximum Likelihood“-Klassifikation als auch „Maximum Likelihood“-Klassifikation auf Segmentbasis verwendet. Anschließend wurden die Genauigkeiten beiden Klassifikationen überprüft und miteinander verglichen.

Eingangsdaten für die Klassifikation waren fusionierte IKONOS- und topographisch normalisierte Landsat TM-Daten. Beide Satellitendaten wurden orthorektifiziert. Die Vorverarbeitungen wurden bereits im vorhergehenden Abschnitt 5.33 erläutert.

\subsection{Klassifikation der IKONOS-Daten}

\subsection{Klassifikationsschema}

Für die Klassifikation sollte zuerst ein geeignetes Klassifikationsschema, in dem alle interessierenden Informationsklassen eindeutig definiert sind, hergestellt werden. Das Klassifikationsschema hängt einerseits von den Informationsbedürfnissen der gestellten Aufgabe und andererseits von dem benutzten Fernerkundungsmaterial ab. Da in der vorliegenden Untersuchung die Verwendbarkeit der Satellitenfernerkundungsdaten zur Kartierung und zur Erfassung von Veränderungen der Waldflächen überprüft werden soll, wurde die koreanische Waldkartierungsvorschrift (Korean Forest Research Institut: KFRI, 1996) für die Erstellung der Waldtypenkarte als Klassifizierungsschema übernommen. Die Waldkartierungsvorschrift ist in die nationalen Waldinventur Koreas implementiert. Durch einen Waldbegang und Studien der Waldtypenkarte wurden die in Frage kommenden Klassen festgelegt. Da in der Waldkartierungsvorschrift nur die Klassen im Waldgebiet definiert worden sind, wurden einige Klassen außerhalb des Waldgebietes als Nichtwaldflächen zusätzlich definiert (Tabelle 5.9). 
Tabelle 5.9: $\quad$ Klassifikationsschema für die vorliegende Untersuchung

\begin{tabular}{|c|c|c|c|}
\hline \multicolumn{3}{|c|}{ Beschreibung der Klassen } & Abkürzung \\
\hline \multirow{7}{*}{$\begin{array}{c}\text { Wald } \\
\text { Klassen }\end{array}$} & \multirow{9}{*}{$\begin{array}{c}\text { Definition der } \\
\text { KFRI }\end{array}$} & Pinus densiflora-Bestände & PD \\
\hline & & Pinus koraiensis-Bestände & PK \\
\hline & & Larix leptolepis-Bestände & PL \\
\hline & & Quercus spp. -Bestände & Q \\
\hline & & Laub-Nadel Mischwald-Bestände & M \\
\hline & & Nadel Mischwald-Bestände & $\mathrm{C}$ \\
\hline & & Kahlflächen / Jungbestände & $\mathrm{F}$ \\
\hline \multirow{5}{*}{$\begin{array}{l}\text { Nichtwald } \\
\text { Klassen }\end{array}$} & & Grassland & LP \\
\hline & & Landwirtschaftlichen Flächen & $\mathrm{L}$ \\
\hline & \multirow{3}{*}{$\begin{array}{c}\text { Eigene } \\
\text { Definition }\end{array}$} & Infrastruktur & Infra \\
\hline & & Wasserflächen & Wasser \\
\hline & & Sonstige vegetationslose Flächen & Rest \\
\hline
\end{tabular}

\subsection{Pixelbasierte „Maximum Likelihood“-Klassifikation}

\section{Auswahl der Referenzflächen}

Um Klassifikationsergebnisse unterschiedlicher methodischer Ansätze zu vergleichen und zu beurteilen, sollen störende Einflußfaktoren möglichst ausgeschlossen sein. Dies gilt auch bei der Auswahl von Referenzflächen(„Ground Truth“ oder Trainingsflächen) für die Klassifikation. Da es sich in der vorliegenden Untersuchung eine um Vergleichstudie mehrerer Klassifikationsmethoden handelt, wäre es sinnvoll, wenn alle herangezogenen Klassifikationen die gleichen Referenzdaten verwenden. Aus diesem Grund erfolgte die Auswahl der Referenzflächen auf der Basis von Segmenten (näheres zur Segmentierung siehe Abschnitt 5.422.1). So wurde ein Segment als Referenzfläche verwendet, wenn es sich innerhalb der terrestrisch angesprochenen Fläche befindet. Zusätzliche Referenzflächen wurden durch eine visuelle Interpretation am Bildschirm mit Hilfe verschiedener Kanalkombinationen ausgewählt, weil die Anzahl der terrestrisch angesprochenen Flächen nicht ausreichend für die Klassifikation und Überprüfung der Klassifikationsergebnisse waren. Dabei wurde festgestellt, daß die 
landwirtschaftlich genutzten Flächen unter den zu klassifizierenden Klassen zu große spektrale Unterschiede aufweisen. Ursächlich dafür sind vor allem unterschiedliche Wassermengen in Böden. So schwankt z.B. die Bodenfeuchtigkeit in einigen Reisfeldern sehr stark. Deswegen wurden die landwirtschaftlich genutzten Flächen in zwei Klassen, feucht $\left(\mathrm{L}_{-} \mathrm{N}\right)$ und trocken $\left(\mathrm{L}_{-} \mathrm{T}\right)$, getrennt. Diese Trennung erfolgte nur durch visuelle Interpretation.

Am Ende standen 260 Referenzflächen (20 Flächen je Klasse) zur Verfügung. Die Referenzflächen wurden dann zufällig in zwei Gruppen zusammengefaßt. Die erste Gruppe wurde zur Klassifizierung die zweite zur Verifizierung der Klassifikation herangezogen.

\section{Signatur Analyse}

Abbildung 5.15 (a) zeigt die Signaturdiagramme von 13 Klassen aus der IKONOSSzene. Die Signaturdiagramme für Waldtypen wurden in Abbildung 5.15 (b) vergrößert dargestellt.

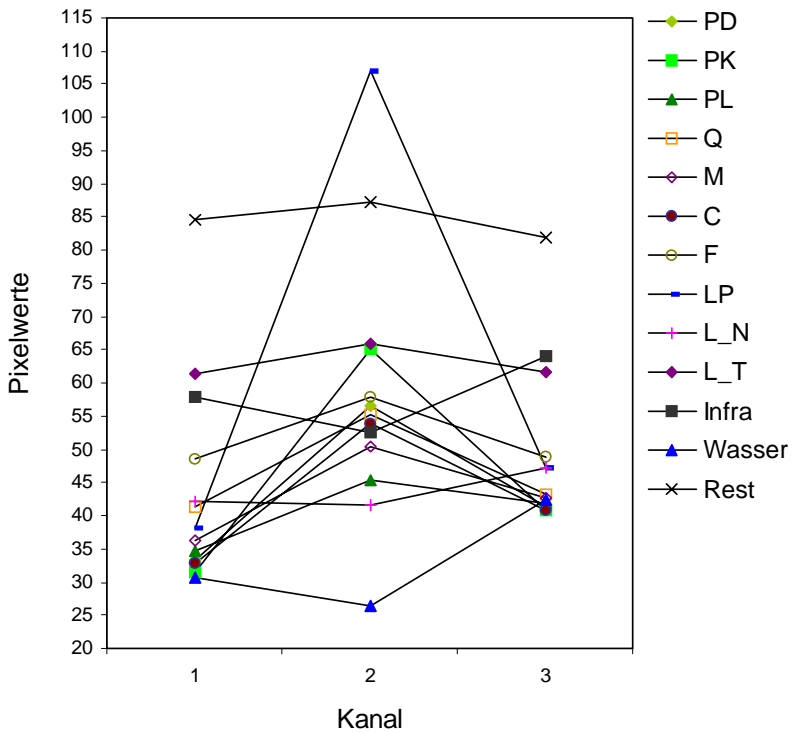

(a)

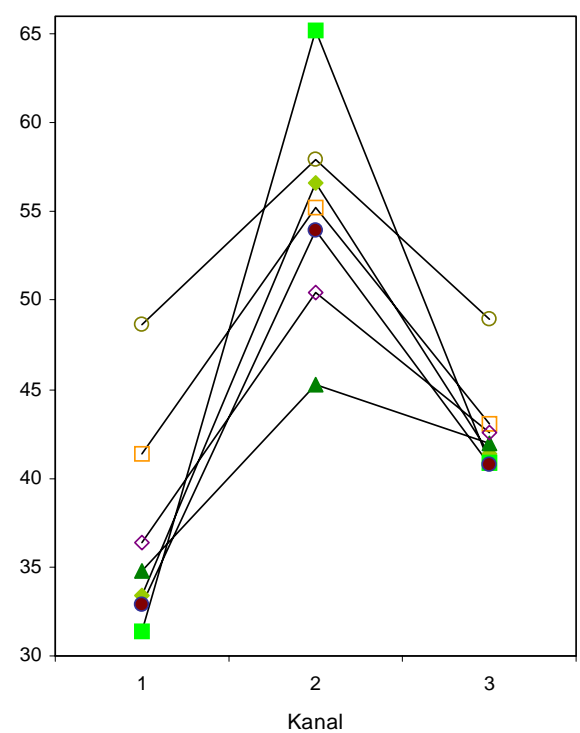

(b)

\section{Abbildung 5.15: Mittlere Spektralsignaturen der fusionierten IKONOS-Szene für} ausgewählte Trainingsflächen verschiedener Landbedeckungsklassen(a) bzw. verschiedener Waldklassen( $b$ ) 
Im allgemeinen zeigten das Signaturdiagramme eine gute Differenzierung zwischen den Klassen. Der zweite Kanal der fusionierten IKONOS-Daten, welcher aus dem Infra-Rot Kanal hergeleitet wurde, zeigt wie erwartet relativ große spektrale Differenzen zwischen den unterschiedlichen Klassen. Hingegen läßt der dritte Kanal (Blau im multispektralen Datensatz) eine Trennung verschiedener Waldtypen nicht zu.

Die Trennung sowohl zwischen den Pinus densiflora Beständen (PD) und NadelMischwäldern (C) als auch Lärchenbestände (PL) und Laub-Nadel-Mischwälder (M) war schwierig. Dies ist möglicherweise auf die Artenzusammensetzung der Mischwaldklassen zurückzuführen. Die Trennbarkeitsmatrix in Tabelle 5.10 zeigt die JefferiesMatusita (JM) Best-Minimum-Distanzen zwischen den verschiedenen Klassen und verdeutlicht die Schwierigkeit bei der spektralen Trennung der beiden Mischwaldtypen. Die Werte der Jefferies-Matusita -Distanzen variieren theoretisch zwischen 0 und 1414 und größere Distanzwerte bedeuten eine bessere Trennbarkeit.

Tabelle 5.10: Trennbarkeit der Klassen in der IKONOS-Szene. (Jefferies-Matusita Best-Minimum Distanz)

\begin{tabular}{c|cccccccccccc}
\hline & PD & PK & PL & Q & M & C & F & LP & L_N & L_T & Infra & Wasser \\
\hline PK & 720 & & & & & & & & & & & \\
PL & 1112 & 1319 & & & & & & & & & & \\
Q & 974 & 1253 & 1059 & & & & & & & & & \\
M & 793 & 1183 & 623 & 707 & & & & & & & & \\
C & 456 & 812 & 934 & 1017 & 724 & & & & & & & \\
F & 1343 & 1400 & 1368 & 1028 & 1280 & 1371 & & & & & & \\
LP & 1414 & 1414 & 1414 & 1414 & 1414 & 1414 & 1414 & & & & & \\
L_N & 1368 & 1404 & 1291 & 1346 & 1280 & 1305 & 1335 & 1414 & & & & \\
L_T & 1372 & 1404 & 1325 & 1331 & 1323 & 1360 & 1140 & 1414 & 1237 & & & \\
Infra & 1409 & 1413 & 1406 & 1404 & 1402 & 1401 & 1377 & 1414 & 1156 & 1244 & & \\
Wasser & 1359 & 1397 & 1312 & 1302 & 1298 & 1315 & 1321 & 1414 & 1206 & 1310 & 1265 & \\
Rest & 1414 & 1414 & 1414 & 1414 & 1414 & 1414 & 1410 & 1414 & 1414 & 1128 & 1359 & 1401 \\
\hline
\end{tabular}

Verifizierung der Ergebnisse der pixelbasierten „Maximum Likelihood“Klassifikation

Nach der „Maximum Likelihood“-Klassifikation auf Pixelebene wurde mit Hilfe der unabhängigen Referenzflächen, wie bereits oben erläutert, eine Verifizierung der Klassifikationsergebnisse durchgeführt. Die Verifizierung wurde in vorliegender 
Untersuchung nicht einzeln pixelweise, sondern flächenweise vorgenommen. Alle Pixel in einer Referenzfläche wurden für die entsprechende Klasse zur Verifikation herangezogen.

In Tabelle 5.11 wurde die Fehlermatrix der Klassifikationsergebnisse der pixelbasierten „Maximum Likelihood“-Methode dargestellt. Die Tabelle gibt die Anzahl der richtig bzw. falsch klassifizierten Pixel in einer Klasse an. Die Waldklassen, d.h. Pinus densiflora (PD), Pinus koraiensis (PK), Larix leptolepis (PL), Quercus spp. (Q), LaubNadel Mischwald (M), Nadel-Mischwald (C) und Kahlfläche / Jungbestände (F), zeigen niedrigere Klassifikationsgenauigkeiten als die Nichtwald-Klassen. Ein möglicher Grund dafür ist neben den ähnlichen mittleren Spektralwerten zwischen den Waldklassen auch die hohe geometrische Auflösung der IKONOS-Daten. Die hohe geometrische Auflösung führt $\mathrm{zu}$ einer hohen Streuung der Pixelwerte sowie zu Fehlklassifikation insbesondere innerhalb einer Waldklasse. Die stärkere Strukturierung der Waldoberflächen im Vergleich zur Nichtwaldfläche ist dafür verantwortlich zu machen. Die Kartierung der Klasse Nadel-Mischwald (C) erreichte eine Herstellergenauigkeit von nur 10,4\%. Sie wurde oftmals als Pinus densiflora- (PD) bzw. Larix leptolepis (PL) Bestände klassifiziert.

Bei der Klasse Grassland erreichte die Klassifikation die höchste Übereinstimmung mit den Referenzflächen mit einer Genauigkeit von über 99\%. Jedoch sind in dieser Klasse einige fehlklassifizierte Pixel zu sehen, obwohl sich ihre Reflektionscharakteristiken deutlich von den anderen Klassen unterscheidet. Dies ist wahrscheinlich auf die fehlerhafte Abgrenzung der Referenzflächen zurückzuführen. 
Tabelle 5.11: Fehlermatrix für die pixelbasierte „Maximum Likelihood“-Klassifikation der IKONOS-Daten.

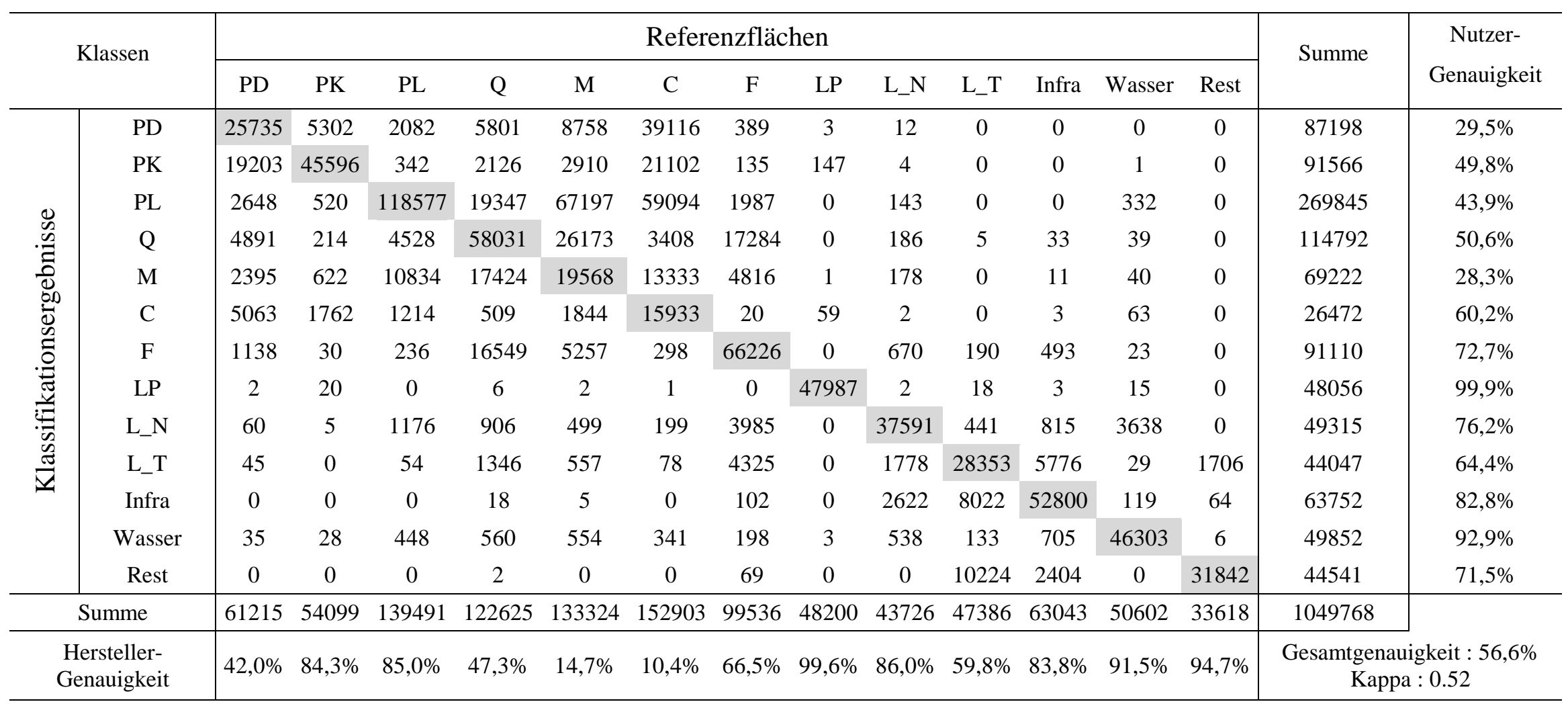




\subsection{Segmentbasierte Klassifikation der IKONOS-Daten}

\subsubsection{Segmentierung}

Die Segmentierung ist der erste Schritt für eine segmentbasierte Klassifikation. Bei der Segmentierung wird das Bild in zahlreiche homogene Teilflächen eingeteilt, welche die Basiseinheiten für eine anschließende Klassifikation bilden. In vielen Fällen wird die Segmentierung nicht zur direkten Extraktion eines letztendlich gewünschten Segmentes führen. Die optimale Segmentgröße kann über mehrere Segmentierungen schrittweise herausgefunden werden. Bei der zur Segmentierung verwendeten Software „eCognition“ ist dies durch die Erstellung verschiedener Level mit verschiedenen Kombinationen von Parametern möglich, wobei die Objekte aus der vorhergehenden Segmentierung als Basisbausteine für die nächste dienen. Die Einflüsse der einzelnen Parameter auf die Segmentbildung wurden bereits im Abschnitt 4.343.3 erläutert.

Nach mehren Erprobungen mit verschiedenen Kombinationen von Parametern wurde eine Strategie für die Segmentierung der IKONOS-Daten erstellt.

Im ersten Schritt der Segmentierung wurden mit einem relativ kleinen „Scale Parameter“ und einer starken Gewichtung des „Shape Parameters“, vor allem des „Compactness Parameters“ gearbeitet. So wurde vermieden, zu stark verzweigte Segmente zu bilden, welche bei einer Flächenkartierung nicht sinnvoll sind.

Im zweiten Schritt der Segmentierung wurde die Gewichtung des „Color Parameters“ verstärkt und der „Scale Parameter“ etwas erhöht. Dabei wurde der „Smoothness“ unter dem „Shape Parameter“ eine stärkere Gewichtung gegeben. Die dadurch resultierenden Segmente sind spektral sehr homogen und von einer relativ naturnahen Form.

Im dritten Schritt wurde dann versucht, eine optimale Segmentgröße für die Klassifikation zu finden. Eine optimale Segmentgröße im Sinne der Klassifikation bedeutet möglichst große Segmente zu bilden, welche die Grenze anderer Klassen nicht überschreiten. Dabei wurde nur der „Scale Parameter“ vergrößert, jedoch darauf geachtet, daß er nicht zu groß wird. Da ein kleiner „Scale Parameter“ nur eine kleine Heterogenität bei der Segmentbildung zuläßt, werden hierdurch die Segmente homogener. Außerdem wird bei kleinen Segmenten die Gefahr geringer, ein Segment mit Pixeln aus verschiedenen Klassen zu erzeugen. 
So wurde in der vorliegenden Untersuchung drei Segmentierungsläufe vollzogen. Tabelle 5.12 zeigt die verwendeten Parameter für die Segmentierung der IKONOSDaten. In Abbildung 5.16 werden daraus resultierte Segmente beispielhaft in einem kleinen Ausschnitt graphisch dargestellt.

Tabelle 5.12: Verwendete Parameter für die Segmentierung der IKONOS-Daten.

\begin{tabular}{cccc}
\hline Level & Scale Parameter & Color / Shape & $\begin{array}{c}\text { Smoothness / } \\
\text { Compactness }\end{array}$ \\
\hline Level 1 & 20 & $0,3 / 0,7$ & $0,2 / 0,8$ \\
Level 2 & 25 & $0,9 / 0,1$ & $0,9 / 0,1$ \\
Level 3 & 75 & $0,9 / 0,1$ & $0,9 / 0,1$ \\
\hline
\end{tabular}

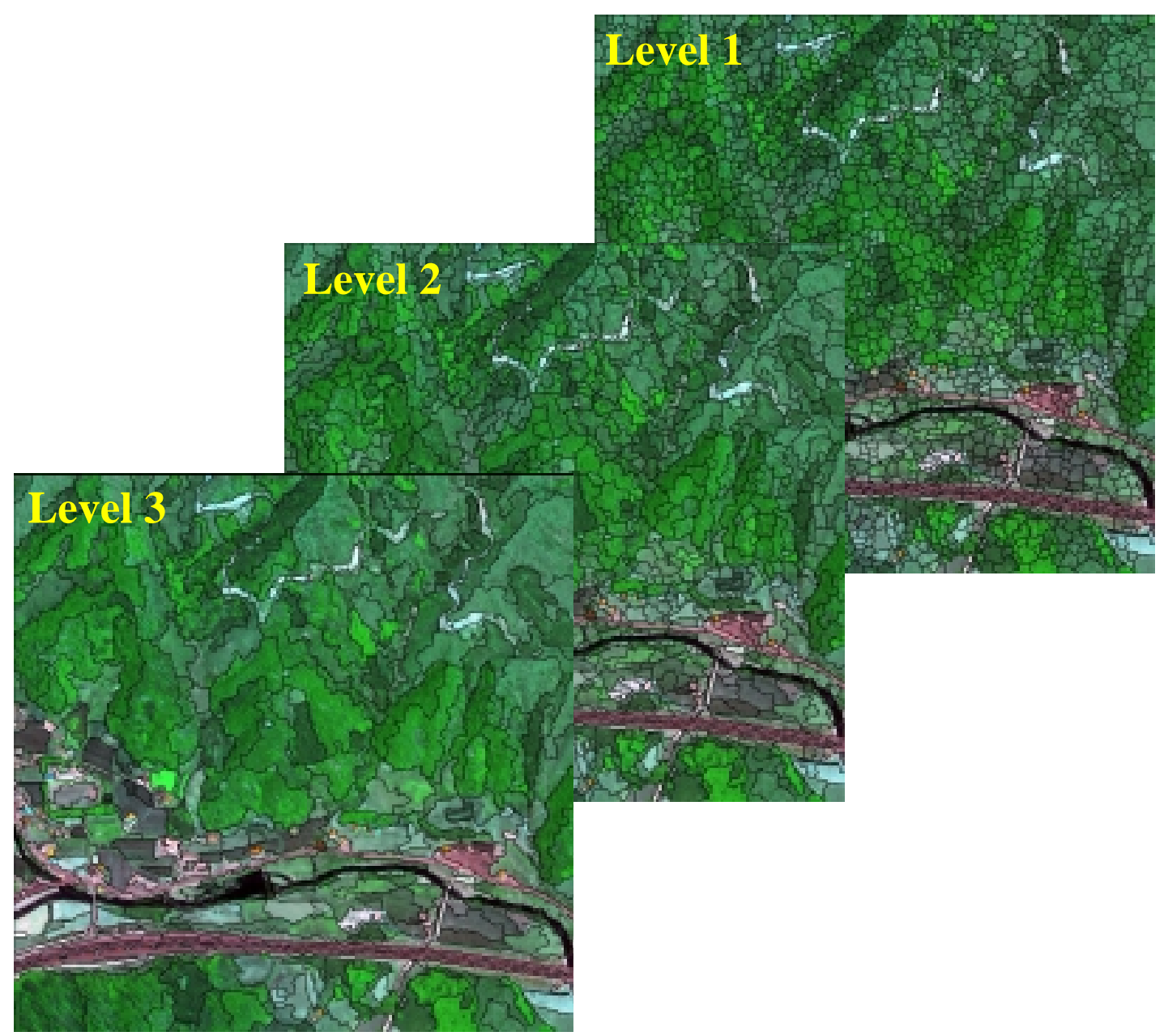

Abbildung 5.16: Segmtierungsergebnisse der IKONOS-Daten 


\subsubsection{Mehrheitszuweisung}

Nach der Segmentierung wurde jedem einzelnen Segment eine eindeutige Klasse aufgrund der Ergebnisse der pixelbasierten „Maximum Likelihood“-Klassifikation nach dem Mehrheitsprinzip zugeordnet.

Die Ergebnisse wurden anhand von unabhängigen Referenzflächen verifiziert (Tabelle 5.13). Durch die Mehrheitszuweisung wurde ein verbessertes Ergebnis mit einer Gesamtgenauigkeit von 65,7\% erreicht. Bei der pixelbasierten „Maximum Likelihood“Klassifikation lag die Gesamtgenauigkeit lediglich bei 56,6\%. Bei den einzelnen Klassen außer den beiden Mischwaldklassen ( $M$ und $C$ ) hatten die Segmente eine eindeutige Klassenzugehörigkeit. Kein Segment wurde den Mischwaldklassen zugeordnet. Dies ist auf die relativ hohe Varianz, bedingt durch die hohe geometrische Auflösung der IKONOS-Daten zurückzuführen (vergl. Abschnitt 5.412)

\subsubsection{Segmentbasierte „Maximum Likelihood“-Klassifikation}

\section{Signaturanalyse}

Bei einer segmentbasierten Klassifikation können segmentspezifische, repräsentative Merkmale bestimmt werden, die eine Klassifikation auf Segmentebene ermöglichen. In der vorliegenden Untersuchung wurde der spektrale Mittelwert und deren Standardabweichung als segmentspezifische Merkmale herangezogen. So stand ein neuer Datensatz mit sechs künstlichen Kanälen (drei Segmentmittelwertkanäle und drei Segmentstandardabweichungskanäle) zur segmentbasierten Klassifikation bereit.

Zur Signaturanalyse wurden die dieselben Trainingsflächen wie bei der pixelbasierten Klassifikation herangezogen. Da die Referenzflächen für die pixelbasierte Klassifikation bereits mit den Segmenten abgegrenzt wurden, weisen die spektralen Signaturen der Segmentmittelwertkanäle die gleichen Werte wie die Signaturen der pixelbasierten Klassifikation auf (siehe Abbildung 5.15). In Abbildung 5.17 wurden die Signaturdiagramme der Standardabweichungen dargestellt. Trotz relativ geringer Unterschiede in den absoluten Werten der Standardabweichungen können jedoch tendenzielle Unterschiede sowohl zwischen den Klassen Pinus densiflora (PD) und Nadel-Mischwald (C) als auch zwischen Laub-Nadelmischwald (M) und Larix leptolepis (PL) festgestellt werden. Eine Trennung dieser Klassen ist also in diesem 
Tabelle 5.13: Fehlermatrix bei einer Mehrheitszuweisung der IKONOS-Daten aufgrund der pixelbasierten „Maximum Likelihood“Klassifikation.

\begin{tabular}{|c|c|c|c|c|c|c|c|c|c|c|c|c|c|c|c|c|}
\hline \multirow{2}{*}{\multicolumn{2}{|c|}{ Klassen }} & \multicolumn{13}{|c|}{ Referenzflächen } & \multirow{3}{*}{$\begin{array}{c}\text { Summe } \\
84046\end{array}$} & \multirow{3}{*}{$\begin{array}{c}\text { Nutzer- } \\
\text { Genauigkeit } \\
56,4 \%\end{array}$} \\
\hline & & \multirow{2}{*}{$\begin{array}{c}\text { PD } \\
47388\end{array}$} & \multirow{2}{*}{$\begin{array}{c}\text { PK } \\
0\end{array}$} & \multirow{2}{*}{$\begin{array}{c}\mathrm{PL} \\
0\end{array}$} & \multirow{2}{*}{$\begin{array}{l}\mathrm{Q} \\
0\end{array}$} & \multirow{2}{*}{$\begin{array}{c}\mathrm{M} \\
0\end{array}$} & \multirow{2}{*}{$\begin{array}{c}\mathrm{C} \\
36658\end{array}$} & \multirow{2}{*}{$\begin{array}{c}\mathrm{F} \\
0\end{array}$} & \multirow{2}{*}{$\begin{array}{c}\text { LP } \\
0\end{array}$} & \multirow{2}{*}{$\frac{\text { L_N }}{0}$} & \multirow{2}{*}{$\frac{\mathrm{L}_{-} \mathrm{T}}{0}$} & \multirow{2}{*}{$\frac{\text { Infra }}{0}$} & \multirow{2}{*}{$\begin{array}{c}\text { Wasse } \\
\text { r } \\
0\end{array}$} & \multirow{2}{*}{$\begin{array}{c}\text { Rest } \\
0\end{array}$} & & \\
\hline \multirow{13}{*}{ 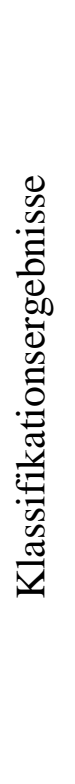 } & $\mathrm{PD}$ & & & & & & & & & & & & & & & \\
\hline & PK & 13827 & 54099 & 0 & 0 & 0 & 8027 & 0 & 0 & 0 & 0 & 0 & 0 & 0 & 75953 & $71,2 \%$ \\
\hline & PL & 0 & 0 & 139491 & 35288 & 121328 & 108218 & 0 & 0 & 0 & 0 & 0 & 0 & 0 & 404325 & $34,5 \%$ \\
\hline & $Q$ & 0 & 0 & 0 & 87337 & 11996 & 0 & 0 & 0 & 0 & 0 & 0 & 0 & 0 & 99333 & $87,9 \%$ \\
\hline & M & 0 & 0 & 0 & 0 & 0 & 0 & 0 & 0 & 0 & 0 & 0 & 0 & 0 & 0 & $0,0 \%$ \\
\hline & $\mathrm{C}$ & 0 & 0 & 0 & 0 & 0 & 0 & 0 & 0 & 0 & 0 & 0 & 0 & 0 & 0 & $0,0 \%$ \\
\hline & $\mathrm{F}$ & 0 & 0 & 0 & 0 & 0 & 0 & 93458 & 0 & 0 & 0 & 0 & 0 & 0 & 93458 & $100,0 \%$ \\
\hline & LP & 0 & 0 & 0 & 0 & 0 & 0 & 0 & 48200 & 0 & 0 & 0 & 0 & 0 & 48200 & $100,0 \%$ \\
\hline & L_N & 0 & 0 & 0 & 0 & 0 & 0 & 6078 & 0 & 43726 & 0 & 0 & 0 & 0 & 49804 & $87,8 \%$ \\
\hline & $\mathrm{L}_{-} \mathrm{T}$ & 0 & 0 & 0 & 0 & 0 & 0 & 0 & 0 & 0 & 28838 & 0 & 0 & 0 & 28838 & $100,0 \%$ \\
\hline & Infra & 0 & 0 & 0 & 0 & 0 & 0 & 0 & 0 & 0 & 10263 & 63043 & 0 & 0 & 73306 & $86,0 \%$ \\
\hline & Wasser & 0 & 0 & 0 & 0 & 0 & 0 & 0 & 0 & 0 & 0 & 0 & 50602 & 0 & 50602 & $100,0 \%$ \\
\hline & Rest & 0 & 0 & 0 & 0 & 0 & 0 & 0 & 0 & 0 & 8285 & 0 & 0 & 33618 & 41903 & $80,2 \%$ \\
\hline \multicolumn{2}{|c|}{ Summe } & 61215 & 54099 & 139491 & 122625 & 133324 & 152903 & 99536 & 48200 & 43726 & 47386 & 63043 & 50602 & 33618 & 1049768 & \\
\hline \multicolumn{2}{|c|}{$\begin{array}{l}\text { Hersteller- } \\
\text { Genauigkeit }\end{array}$} & $77,4 \%$ & $100,0 \%$ & $100,0 \%$ & $71,2 \%$ & $0,0 \%$ & $0,0 \%$ & $93,9 \%$ & $100,0 \%$ & $100,0 \%$ & $60,9 \%$ & $100,0 \%$ & $100,0 \%$ & $100,0 \%$ & \multicolumn{2}{|c|}{$\begin{array}{c}\text { Gesamtgenauigkeit : } \\
65,7 \% \\
\text { Kappa : } 0.62\end{array}$} \\
\hline
\end{tabular}


Fall möglich, wie auch die „Jefferies-Matusita“-Distanzwerte bestätigen (Tabelle 5.14). Die Trennbarkeit zwischen den o.g. Klassen waren zwar am niedrigsten unter den Klassen, aber im Vergleich zu den Trennbarkeiten bei der Pixelbasierte Klassifikation sehr hoch (siehe Tabelle 5.10).

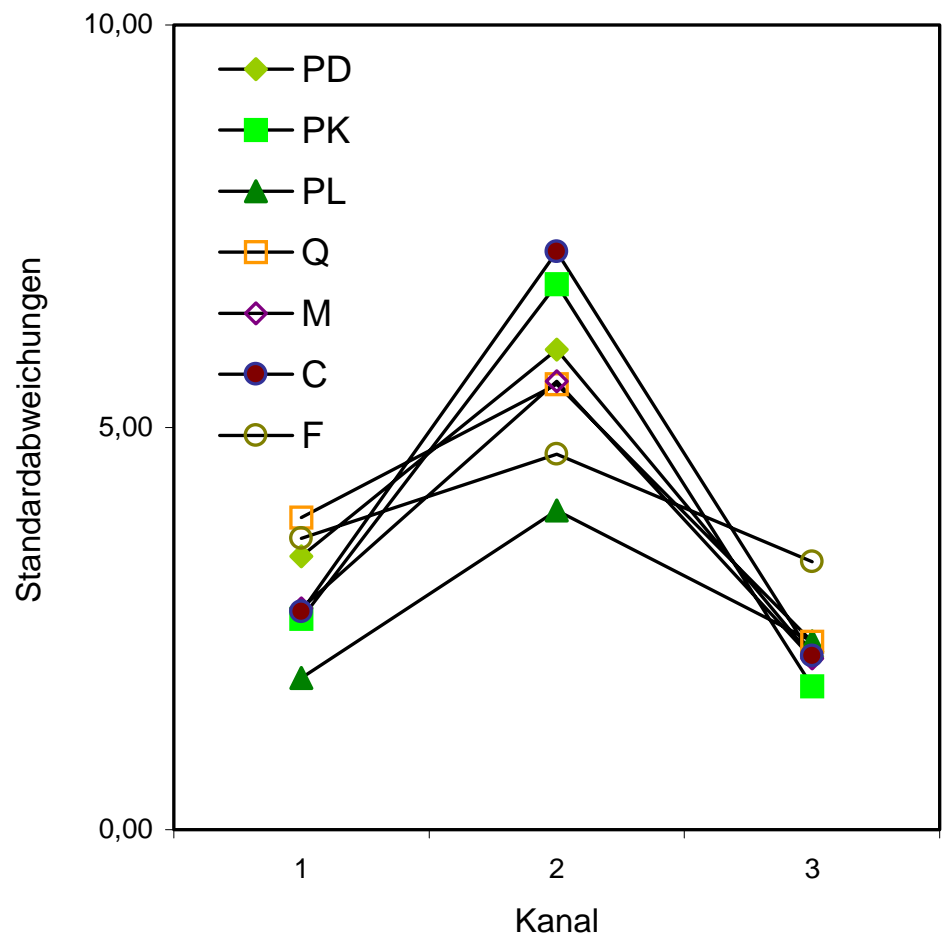

Abbildung 5.17: Mittlere Standardabweichungen der IKONOS-Szene auf der Segmentbasis.

Tabelle 5.14: Trennbarkeit der Klassen in der IKONOS-Szene (Jefferies-Matusita Best-Minimum Distanz). Berechnet wurden auf der Segmentbasis.

\begin{tabular}{c|cccccccccccc}
\hline & PD & PK & PL & Q & M & C & F & LP & L_N & L_T & Infra & Wasser \\
\hline PK & 1379 & & & & & & & & & & & \\
PL & 1411 & 1414 & & & & & & & & & & \\
Q & 1385 & 1414 & 1381 & & & & & & & & & \\
M & 1388 & 1414 & 1308 & 1255 & & & & & & & & \\
C & 1323 & 1386 & 1396 & 1387 & 1355 & & & & & & & \\
F & 1414 & 1414 & 1414 & 1403 & 1414 & 1414 & & & & & & \\
LP & 1414 & 1414 & 1414 & 1414 & 1414 & 1414 & 1414 & & & & & \\
L_N & 1414 & 1414 & 1414 & 1414 & 1414 & 1411 & 1414 & 1414 & & & & \\
L_T & 1414 & 1414 & 1401 & 1414 & 1411 & 1412 & 1395 & 1414 & 1406 & & & \\
Infra & 1414 & 1414 & 1414 & 1414 & 1414 & 1414 & 1414 & 1414 & 1406 & 1414 & & \\
Wasser & 1414 & 1414 & 1414 & 1414 & 1414 & 1414 & 1414 & 1414 & 1400 & 1414 & 1397 & \\
Rest & 1414 & 1414 & 1414 & 1414 & 1414 & 1414 & 1414 & 1414 & 1414 & 1370 & 1412 & 1414 \\
\hline
\end{tabular}




\section{Verifizierung der Ergebnisse der Segmentbasierten „Maximum Likelihood“- Klassifikation}

Die Verifizierungsergebnisse der Klassifikation sind in Tabelle 5.15 in Form einer Fehlermatrix angegeben. Mit der Methode der segmentbasierten „Maximum Likelihood“-Klassifikation wurden eine Gesamtgenauigkeit von 81,9 \% und ein „Kappawert“ von 0.8 erreicht.

Die zwei Mischwald-Klassen (M und C), welche mit der vorhergehenden Methode nicht von den anderen Klassen zu trennen waren, konnten mit einer Nutzer-Genauigkeit von $85,4 \%$ bzw. 84,1 \% klassifiziert wurden. Aus Sicht der Hersteller wurde die Klasse Nadel-Mischwald (C) mit einer Genauigkeit von 57,7\% klassifiziert. Die niedrigste Genauigkeit wurde bei landwirtschaftlich genutzten, trockenen Flächen (L_T) mit einer Genauigkeit von ca. $54 \%$ erreicht. Durch Zusammenfassung der beiden landwirtschaftlichen Flächen ergibt sich eine Herstellergenauigkeit von 76,7 \% und eine Nutzergenauigkeit von $75,8 \%$.

\subsection{Beurteilung und Analyse der Klassifikationsergebnisse der IKONOS-Daten.}

Die Klassifikationsergebnisse der drei erprobten Methoden sind in Tabelle 5.16 noch einmal vergleichend zusammengefaßt. Unter den verwendeten Methoden wurde die Methode „Segmentbasierte-Maximum-Likelihood-Klassifikation“ mit einer Gesamtgenauigkeit von ca. $82 \%$ als best geeignete Methode nachgewiesen.

Tabelle 5.15: Vergleich der Klassifikationsergebnisse der verschiedenen Methoden

\begin{tabular}{ccc}
\hline Methode & Gesamtgenauigkeit & Kappa \\
\hline Pixelbasierte MLK & $57 \%$ & 0,52 \\
Mehrheitszuweisung & $66 \%$ & 0,62 \\
Segmentbasierte MLK & $82 \%$ & 0,80 \\
\hline
\end{tabular}

Das Hauptinteresse der Klassifikation der hoch auflösenden Satellitendaten war in der vorliegenden Untersuchung die Überprüfung der Verwendbarkeit von IKONOS-Daten für forstliche Zwecke, vor allem zur Kartierung von Waldtypen. Daher wurden die nicht zu Wald gehörenden Klassen in einer Klasse zusammengefaßt, und die Genauigkeit der Klassifikation noch einmal hergeleitet. 
Tabelle 5.16: Fehlermatrix für die segmentbasierte „Maximum Likelihood“-Klassifikation der IKONOS-Daten.

\begin{tabular}{|c|c|c|c|c|c|c|c|c|c|c|c|c|c|c|c|c|}
\hline \multirow{2}{*}{\multicolumn{2}{|c|}{ Klassen }} & \multicolumn{13}{|c|}{ Referenzflächen } & \multirow{3}{*}{$\begin{array}{c}\text { Summe } \\
77871\end{array}$} & \multirow{3}{*}{\begin{tabular}{|c|} 
Nutzer- \\
Genauigkeit
\end{tabular}} \\
\hline & & \multirow{2}{*}{\begin{tabular}{|l|} 
PD \\
42681
\end{tabular}} & \multirow{2}{*}{$\frac{\mathrm{PK}}{0}$} & \multirow{2}{*}{$\begin{array}{c}\mathrm{PL} \\
0\end{array}$} & \multirow{2}{*}{$\frac{\mathrm{Q}}{0}$} & \multirow{2}{*}{$\begin{array}{c}\mathrm{M} \\
8343\end{array}$} & \multirow{2}{*}{$\frac{C}{26847}$} & \multirow{2}{*}{$\begin{array}{c}\mathrm{F} \\
0\end{array}$} & \multirow{2}{*}{$\begin{array}{c}\text { LP } \\
0\end{array}$} & \multirow{2}{*}{$\frac{\text { L_N }}{0}$} & \multirow{2}{*}{$\frac{\mathrm{L}_{-} \mathrm{T}}{0}$} & \multirow{2}{*}{$\begin{array}{c}\text { Infra } \\
0\end{array}$} & \multirow{2}{*}{$\begin{array}{c}\text { Wasser } \\
0\end{array}$} & \multirow{2}{*}{$\frac{\text { Rest }}{0}$} & & \\
\hline \multirow{13}{*}{ 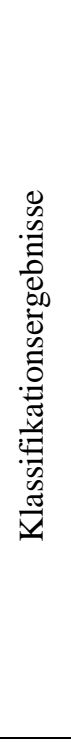 } & PD & & & & & & & & & & & & & & & \\
\hline & PK & 5205 & 54099 & 0 & 12975 & 0 & 8027 & 0 & 0 & 0 & 0 & 0 & 0 & 0 & 80306 & $67,4 \%$ \\
\hline & PL & 0 & 0 & 139491 & 0 & 17278 & 14536 & 0 & 0 & 0 & 0 & 0 & 0 & 0 & 171305 & $81,4 \%$ \\
\hline & Q & 0 & 0 & 0 & 89256 & 0 & 15253 & 0 & 0 & 0 & 0 & 0 & 0 & 0 & 104509 & $85,4 \%$ \\
\hline & M & 0 & 0 & 0 & 20394 & 107703 & 0 & 0 & 0 & 0 & 0 & 0 & 0 & 0 & 128097 & $84,1 \%$ \\
\hline & $\mathrm{C}$ & 13329 & 0 & 0 & 0 & 0 & 88240 & 0 & 0 & 0 & 0 & 0 & 0 & 0 & 101569 & $86,9 \%$ \\
\hline & $\mathrm{F}$ & 0 & 0 & 0 & 0 & 0 & 0 & 79848 & 0 & 0 & 0 & 0 & 0 & 0 & 79848 & $100,0 \%$ \\
\hline & LP & 0 & 0 & 0 & 0 & 0 & 0 & 0 & 48200 & 0 & 0 & 0 & 0 & 0 & 48200 & $100,0 \%$ \\
\hline & $\mathrm{L} \_\mathrm{N}$ & 0 & 0 & 0 & 0 & 0 & 0 & 0 & 0 & 40156 & 4270 & 0 & 0 & 0 & 44426 & $90,4 \%$ \\
\hline & L_T & 0 & 0 & 0 & 0 & 0 & 0 & 19688 & 0 & 0 & 25494 & 0 & 0 & 2577 & 47759 & $53,4 \%$ \\
\hline & Infra & 0 & 0 & 0 & 0 & 0 & 0 & 0 & 0 & 0 & 0 & 63043 & 0 & 0 & 63043 & $100,0 \%$ \\
\hline & Wasser & 0 & 0 & 0 & 0 & 0 & 0 & 0 & 0 & 3570 & 5993 & 0 & 50602 & 0 & 60165 & $84,1 \%$ \\
\hline & Rest & 0 & 0 & 0 & 0 & 0 & 0 & 0 & 0 & 0 & 11629 & 0 & 0 & 31041 & 42670 & $72,8 \%$ \\
\hline \multicolumn{2}{|c|}{ Summe } & 61215 & 54099 & 139491 & 122625 & 133324 & 152903 & 99536 & 48200 & 43726 & 47386 & 63043 & 50602 & 33618 & 1049768 & \\
\hline & $\begin{array}{l}\text { eller- } \\
\text { igkeit }\end{array}$ & $69,7 \%$ & $100,0 \%$ & $100,0 \%$ & $72,8 \%$ & $80,8 \%$ & $57,7 \%$ & $80,2 \%$ & $100,0 \%$ & $91,8 \%$ & $53,8 \%$ & $100,0 \%$ & $100,0 \%$ & $92,3 \%$ & \multicolumn{2}{|c|}{$\begin{array}{c}\text { Gesamtgenauigkeit : } \\
81,9 \% \\
\text { Kappa : } 0,8\end{array}$} \\
\hline
\end{tabular}


In Tabelle 5.17 sind die Ergebnisse mit der Methode „Segmentbasierte Maximum Likelihood Klassifikation" aus der Sicht von Hersteller in prozentualen Werten angeben. Dabei wurde eine Gesamtgenauigkeit von ca. $85 \%$ erreicht. Das Ergebnis ist in Form einer Karte in Abbildung 5.18 dargestellt.

Tabelle 5.17: Klassifikationsergebnis der IKONOS-Daten zur Kartierung von Waldtypen. Die Angaben sind Prozentuale Werte aus der Sicht von Hersteller.

\begin{tabular}{c|cccccccc}
\hline Klasse & PD & PK & PL & Q & M & C & F & $\begin{array}{c}\text { Nicht- } \\
\text { Wald }\end{array}$ \\
\hline PD & 69,7 & - & - & - & 6,3 & 17,6 & - & - \\
PK & 8,5 & 100 & - & 10,6 & - & 5,2 & - & - \\
PL & - & - & 100 & - & 13 & 9,5 & - & - \\
Q & - & - & - & 72,8 & - & 10,0 & - & - \\
M & - & - & - & 16,6 & 80,8 & - & - & - \\
C & 21,8 & - & - & - & - & 57,7 & - & - \\
F & - & - & - & - & - & - & 80,2 & - \\
Nicht-Wald & - & - & - & - & - & - & 19,8 & 100 \\
\hline
\end{tabular}

\section{Berechnung einer Flächenbilanz}

Aus den Klassifikationsergebnissen wurde eine Flächenbilanz nach Waldtypen berechnet und in Tabelle 5.18 dargestellt. Der Waldflächenanteil im Untersuchungsgebiet beträgt ca. $78 \%$. Keine dominierende Baumart ist zu erkennen. Etwa $51 \%$ der Waldflächen ist mit Nadelbaumarten bedeckt. Laubwälder (Q) und Mischwälder nehmen jeweils ca. 20 \% der gesamten Waldflächen ein. Die Kahlflächen / Jungbestände (ca. 9,6 \% der Waldfläche) sind fast alle wegen Insektenschäden (Gallmücke) geschlagene Flächen, die früher mit Pinus densiflora bestockt waren. Daher könnten diese Flächen als Insektenschadflächen angesehen werden. 


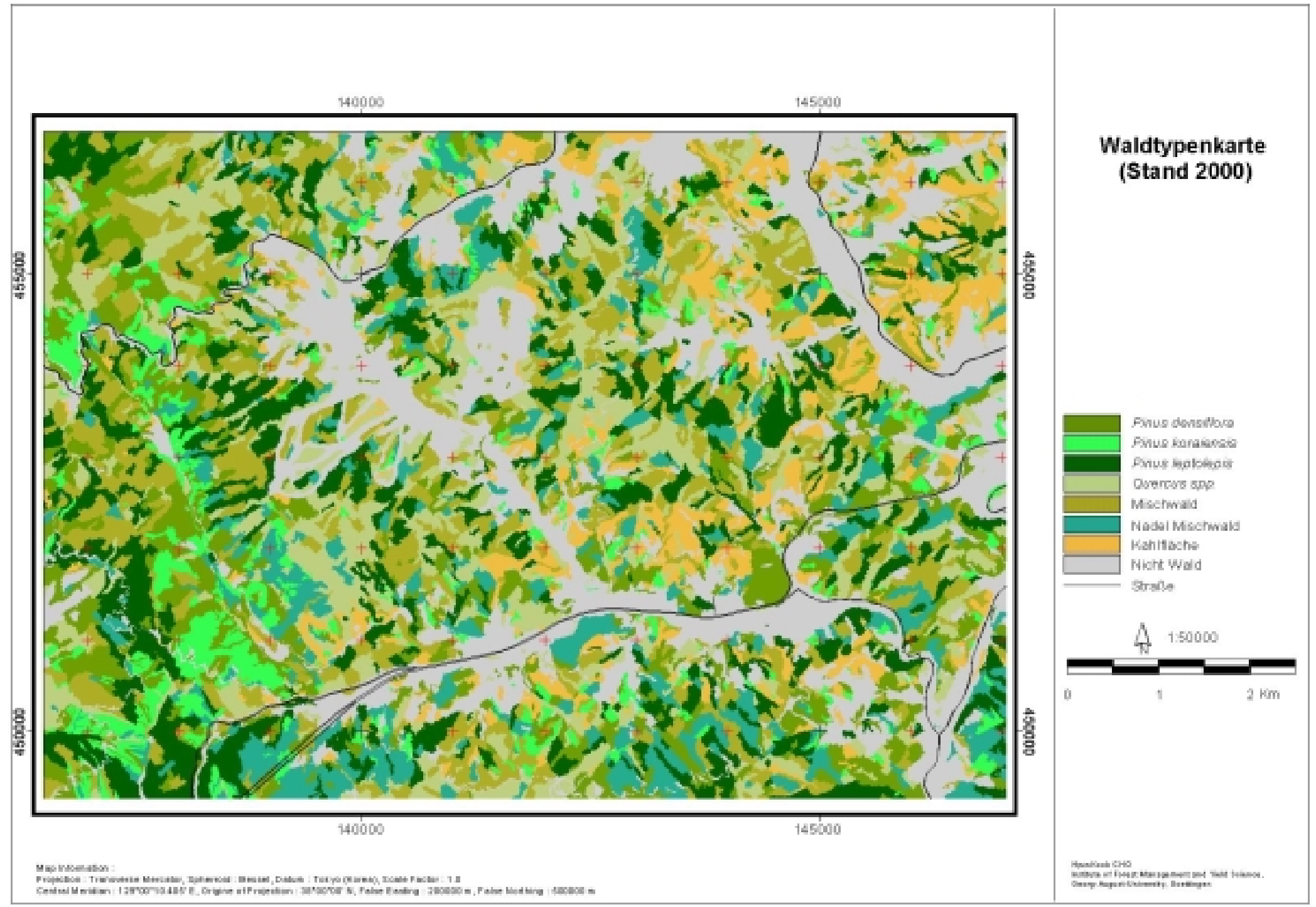

Abbildung 5.18: Waldtypenkarte des Untersuchungsgebietes erstellt aus der Klassifikation der IKONOS-Daten von 2000 
Tabelle 5.18: Flächenbilanz der Klassifikation der IKONOS-Daten.

\begin{tabular}{|c|c|c|c|}
\hline \multirow{2}{*}{ Waldtypen } & \multirow{2}{*}{$\begin{array}{l}\text { Flächengröße } \\
\text { (ha) }\end{array}$} & \multicolumn{2}{|c|}{ Flächen Anteil (\%) } \\
\hline & & Gesamtfläche & Waldfläche \\
\hline Pinus densiflora $(P D)$ & 720,5 & 9,4 & 12,1 \\
\hline Pinus koraiensis (PK) & 493,0 & 6,4 & 8,3 \\
\hline Larix leptolepis $(P L)$ & 1080,7 & 14,1 & 18,2 \\
\hline Quercus spp. (Q) & 1157,6 & 15,1 & 19,5 \\
\hline Laub-Nadel Mischwald (M) & 1213,2 & 15,8 & 20,4 \\
\hline Nadel Mischwald (C) & 713,7 & 9,3 & 12,0 \\
\hline Kahlfläche / Jungbestände (F) & 569,5 & 7,4 & 9,6 \\
\hline Nicht-Wald & 1727,8 & 22,5 & - \\
\hline Gesamt & 7676,0 & 100 & 100 \\
\hline
\end{tabular}

\subsection{Klassifikation der Landsat TM-Daten}

Für die Klassifikation der Landsat TM-Daten wurde die gleiche Vorgehensweise wie bei den IKONOS-Daten angewandt. Im folgenden Abschnitt wird die Klassifikation der Landsat TM-Daten von 1992 und deren Ergebnisse mit besonderer Betonung der abweichenden Punkte von der Klassifikation der IKONOS-Daten angewandten Methodik erläutert.

\subsection{Pixelbasierte ,Maximum Likelihood“-Klassifikation}

\section{Auswahl der Referenzflächen}

Die Gewinnung der Referenzinformationen erfolgte durch eine Stereoauswertung der Luftbilder (siehe Abschnitt 5.52). Dabei wurde festgestellt, daß im Untersuchungsgebiet die Klassen Kahlflächen / Jungbestände (F) und Grassland (LP) 1992 nicht existierten. Daher wurden die beiden Klassen aus der Klassifikation ausgeschlossen. Darüber hinaus waren die Klassen „Infrastruktur (Infra)“ und „Sonstige vegetationslose Flächen (Rest)“ nicht im Satellitenbild zu erkennen. In den meisten Fällen wurden diese Klassen in der Klasse der landwirtschaftlichen Flächen (L) zusammengefaßt, weil diese nur kleinflächig vorkommen und wegen der relativ groben Auflösung der TM-Daten sog. Mischpixel mit einer landwirtschaftlich genutzten Fläche gebildet wurden. So blieben acht Klassen für weitere Analysen übrig. 
Die Flächen, die als Referenzflächen herangezogen wurden, wurden wie bei der Klassifizierung von IKONOS-Daten mit einem Segment abgegrenzt.

\section{Signatur Analyse}

In Tabelle 5.19 wurde die Trennbarkeit der acht Klassen mit den Jefferies-Matusita Distanzen angegeben. Die Trennbarkeitsmatrix zeigt im Vergleich zur Klassifikation der IKONOS-Daten eine viel geringere Distanz zwischen den Klassen (vergleiche Tabelle 5.10). Vor allem sind die Distanzwerte der beiden Mischwald-Klassen LaubNadel-Mischwald (M) und Nadel-Mischwald (C) mit Quercus spp. (Q) bzw. Pinus densiflora (PD) besonders klein.

Tabelle 5.19: Trennbarkeit der Klassen auf der Landsat TM-Szene mit der JefferiesMatusita Distanz.

\begin{tabular}{cccccccc}
\hline & PD & PK & PL & Q & M & C & L \\
\hline PK & 840,9 & & & & & & \\
PL & 958,6 & 1113,5 & & & & & \\
Q & 1117,2 & 1031,6 & 634,8 & & & & \\
M & 1072,1 & 952,5 & 655,7 & 459,5 & & & \\
C & 471,3 & 862,4 & 861,6 & 955,4 & 924,1 & & \\
L & 1258,3 & 1255,1 & 1067,7 & 1013,0 & 1036,3 & 1187,8 & \\
Wasser & 1209,2 & 1306,8 & 1131,0 & 1171,2 & 1211,7 & 1155,6 & 990,7 \\
\hline
\end{tabular}

Die Signaturdiagramme von verschiedenen Waldklassen sind in Abbildung 5.19 dargestellt. Die Spektralsignaturen der Waldtypen unterscheiden sich im sichtbaren Bereich nicht von einander, während im Infra-rot Bereich eine bessere Trennungsmöglichkeit existirt. Dennoch lassen sich die beiden Mischwaldklassen nicht von den Pinus densiflora- bzw. Quercus spp.-Beständen trennen. Um die Differenzierungsprobleme der beiden Mischwaldtypen zu verdeutlichen, sind in Abbildung 5.20 die Streuungsellipsen für die Kanäle 4 und 5 dargestellt. Aufgrund der Spektralwerte sind die beiden Mischwald-Klassen nicht zu trennen. Daher wurde die Laub-Nadel-Mischwald-Klasse mit der Quercus spp.-Klasse und die Nadel-MischwaldKlasse mit der Pinus densiflora-Klasse zusammengefaßt. Insgesamt wurden dann nur sechs Klassen zur Klassifikation der Landsat TM-Daten ausgewiesen. 


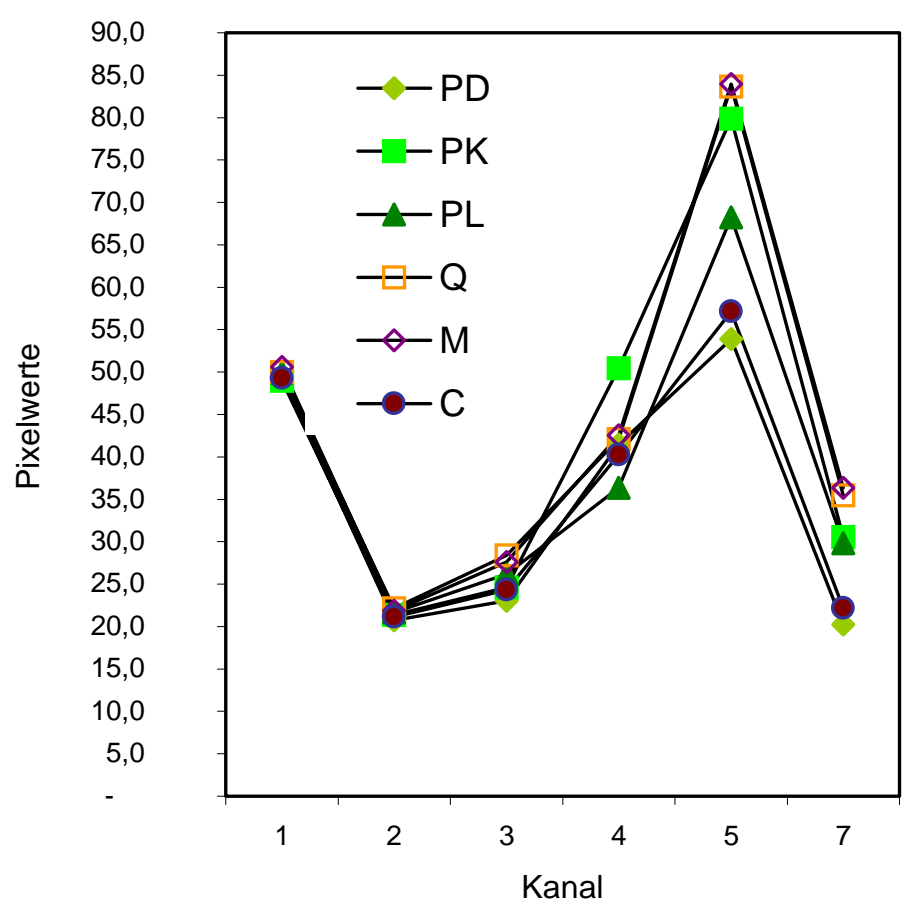

Abbildung 5.19: Mittlere Spektralsignaturen verschiedener Waldklassen anhand ausgewählter Trainingsflächen auf der Landsat TM-Szene

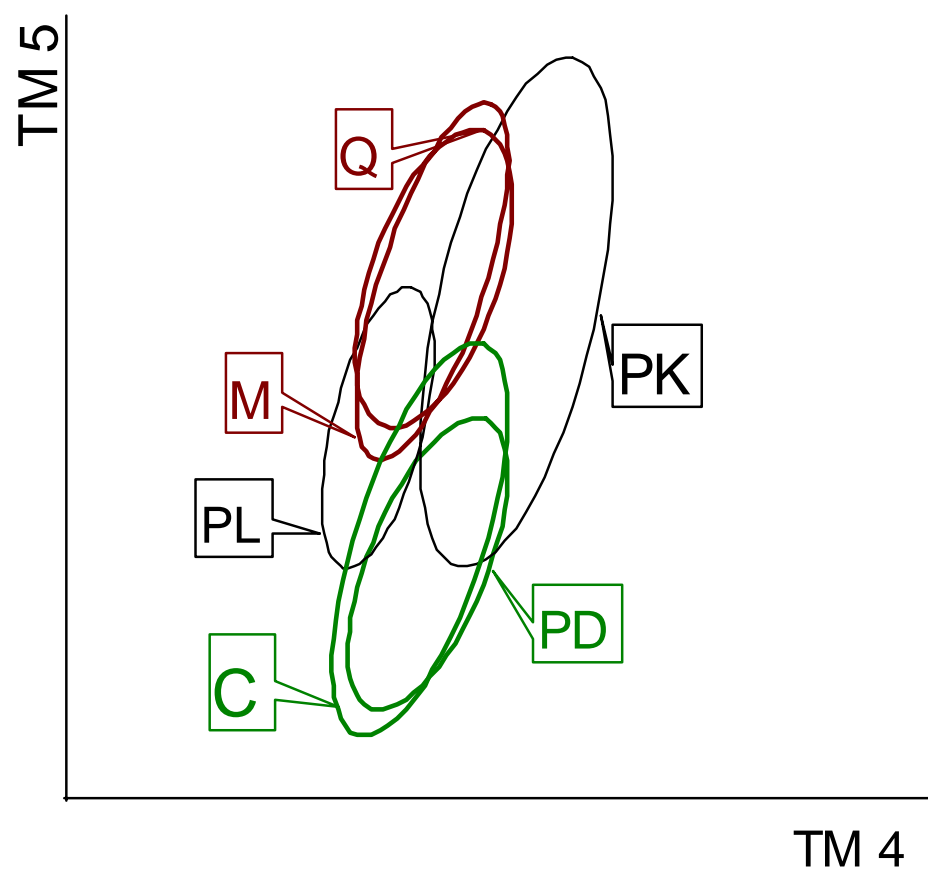

Abbildung 5.20: Die Streuungsellipsen ( \pm zweifache Standardabweichung) verschiedener Waldtypen im zweidimensionalen Merkmalsraum. 


\section{Ergebnisse der pixelbasierten „Maximum Likelihood“"-Klassifikation}

Die Verifizierungsergebnisse der pixelbasierten „Maximum Likelihood“-Klassifikation der Landsat TM-Daten sind in Tabelle 5.20 in Form einer Fehlermatrix dargestellt. Trotz der teilweise unscharfen Trennbarkeiten wurde eine Klassifikation mit einer Gesamtgenauigkeit von 67,3\% und einem Kappawert von 0.59 erreicht. Hier traten die größten Fehler bei der Klasse Wasser auf. Viele Pixel, die eigentlich landwirtschaftlich genutzten Flächen zugehörig sind, wurden als Wasser klassifiziert. Ein Grund dafür ist vor allem der hohe Wassergehalt der Böden von Reisfeldern. Eine weitere häufige Fehlklassifikation war die Klassifikation von PL zu Q / M. Als Ursache für diese Fehlklassifikationen sind wahrscheinlich sowohl die Artenzusammensetzung bei der Klasse Laub-Nadel Mischwald (M) als auch Nadelverfärbungen an Larix leptolepis (PL) anzusehen.

Tabelle 5.20: Fehlermatrix für die pixelbasierte „Maximum Likelihood“-Klassifikation der Landsat TM-Daten. Angegeben ist jeweils die Anzahl von Pixeln.

\begin{tabular}{|c|c|c|c|c|c|c|c|c|c|}
\hline \multirow{2}{*}{\multicolumn{2}{|c|}{ Klasse }} & \multicolumn{6}{|c|}{ Referrenz } & \multirow{2}{*}{ Summe } & \multirow{2}{*}{$\begin{array}{c}\text { Nutzer- } \\
\text { Genauigkeit }\end{array}$} \\
\hline & & $\mathrm{PD} / \mathrm{C}$ & PK & PL & $\mathrm{Q} / \mathrm{M}$ & $\mathrm{L}$ & Wasser & & \\
\hline \multirow{6}{*}{ 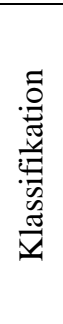 } & $\mathrm{PD} / \mathrm{C}$ & 1282 & 214 & 182 & 17 & 6 & 0 & 1701 & $75,4 \%$ \\
\hline & PK & 106 & 539 & 29 & 75 & 8 & 0 & 757 & $71,2 \%$ \\
\hline & PL & 311 & 12 & 1126 & 253 & 9 & 0 & 1711 & $65,8 \%$ \\
\hline & $\mathrm{Q} / \mathrm{M}$ & 65 & 52 & 454 & 654 & 109 & 1 & 1335 & $49,0 \%$ \\
\hline & $\mathrm{L}$ & 6 & 0 & 56 & 29 & 956 & 5 & 1052 & $90,9 \%$ \\
\hline & Wasser & 38 & 4 & 48 & 5 & 168 & 99 & 362 & $27,3 \%$ \\
\hline \multicolumn{2}{|c|}{ Summe } & 1808 & 821 & 1895 & 1033 & 1256 & 105 & 6918 & \\
\hline & $\begin{array}{l}\text { ller - } \\
\text { igkeit }\end{array}$ & $70,9 \%$ & $65,7 \%$ & $59,4 \%$ & $63,3 \%$ & $76,1 \%$ & $94,3 \%$ & \multicolumn{2}{|c|}{$\begin{array}{c}\text { Gesamt-Genauigkeit } \\
67,3 \% \\
\text { Kappa }: 0.59\end{array}$} \\
\hline
\end{tabular}

\subsection{Segmentbasierte Klassifikation von Landsat TM-Daten}

\subsubsection{Segmentierung}

Bei der Segmentierung der Landsat TM-Daten wurde zuerst die selbe Strategie wie bei den IKONOS-Daten angewand. Dabei wurde festgestellt, daß eine andere Strategie für Landsat TM-Daten nötig ist. Die Landsat TM-Daten besitzen eine relativ grobe geometrische Auflösung von $30 \mathrm{~m}$, die viel geringer als die geometrische Auflösung der IKONOS-Daten ist. Daher weist ein Pixel in der Landsat TM-Szene ähnliche spektrale 
Eigenschaften wie ein Segment aus 30 x 30 Pixeln in der IKONOS-Szene auf. Darüber hinaus weist das Untersuchungsgebiet eine sehr kleinräumige Flächenstruktur auf. Daher wurde auf den ersten Schritt bei der Segmentierungsstrategie der IKONOS-Szene verzichtet. Im ersten Schritt wurde der „Scale Parameter“ gleich dem Wert 3 mit einer Gewichtung von 0,2 für den „Shape Parameter“ und einer Gewichtung von $80 \%(0,8)$ für den Parameter „Compactness“ gesetzt. Die daraus resultierenden Segmente bestehen aus durchschnittlich 7 Pixeln. Der zweite Schritt der Segmentierung wurde mit einem „Scale Parameter“ von 10 durchgeführt. Dabei lag die Gewichtung nur auf dem Parameter „Color“, d.h. die Form der Segmente wurde nicht berücksichtigt.

In Tabelle 5.10 sind die verwendeten Segmentierungsparameter für die Landsat TMDaten zusammengefaßt und in Abbildung 5.21 daraus resultierende Ergebnisse graphisch dargestellt.

Tabelle 5.21: Die verwendeten Parameter für die Segmentierung der Landsat TMDaten.

\begin{tabular}{cccc}
\hline Level & Scale Parameter & Color / Shape & $\begin{array}{c}\text { Smoothness / } \\
\text { Compactness }\end{array}$ \\
\hline Level 1 & 3 & $0,8 / 0,2$ & 0,2 / 0,8 \\
Level 2 & 7 & $1,0 / 0,0$ & $-/-$ \\
\hline
\end{tabular}

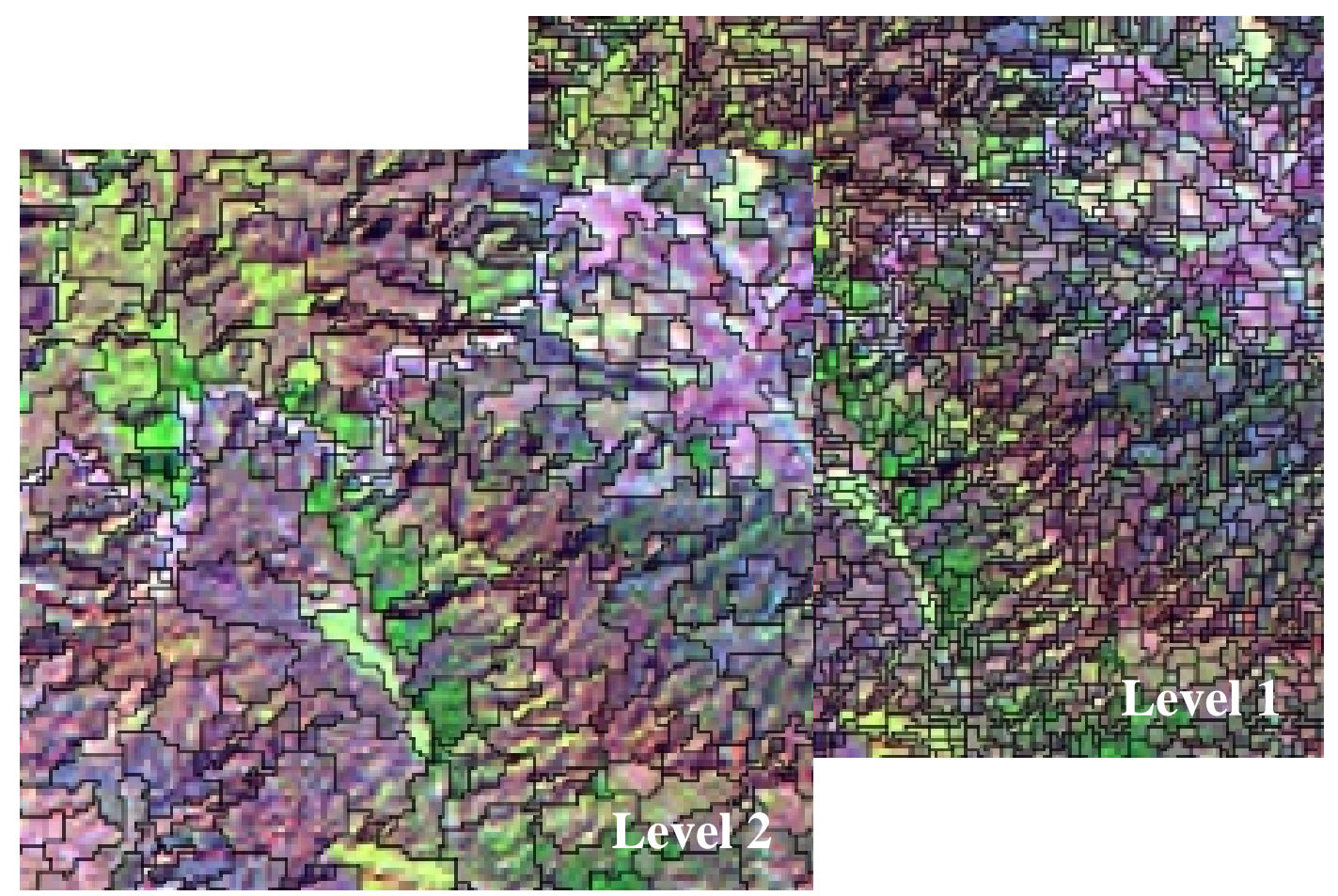

Abbildung 5.21: Segmentierungsergebnisse der Landsat TM-Daten 


\subsubsection{Mehrheitszuweisung}

Die Oben beschriebenen Segmente wurden mit der Methode der Mehrheitszuweisung klassifiziert. Die Verifizierung der Klassifikation erfolgte anhand von unabhängigen Referenzflächen. Die Fehlermatrix zeigt im Vergleich zur pixelbasierten Klassifikation verbesserte Ergebnisse mit einer Gesamtgenauigkeit von 90,1\%. Der Kappawert beträgt dabei 0,87. Die niedrigste Nutzergenauigkeit wurde bei der Klasse Wasser mit ca. $74 \%$ festgestellt. Die häufigste fälschlicherweise als Wasser angesprochene Klasse war landwirtschaftliche Fläche (L), höchstwahrscheinlich Naß-Reisfelder.

Tabelle 5.22: Fehlermatrix bei einer Mehrheitszuweisung der Landsat TM-Daten aufgrund der pixelbasierten „Maximum Likelihood“-Klassifikation.

\begin{tabular}{|c|c|c|c|c|c|c|c|c|c|}
\hline \multirow{2}{*}{\multicolumn{2}{|c|}{ Klasse }} & \multicolumn{6}{|c|}{ Referrenz } & \multirow{2}{*}{ Summe } & \multirow{2}{*}{$\begin{array}{c}\text { Nutzer- } \\
\text { Genauigkeit }\end{array}$} \\
\hline & & $\mathrm{PD} / \mathrm{C}$ & PK & PL & $\mathrm{Q} / \mathrm{M}$ & $\mathrm{L}$ & Wasser & & \\
\hline \multirow{6}{*}{ 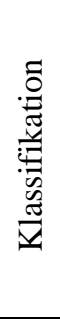 } & $\mathrm{PD} / \mathrm{C}$ & 1808 & 257 & 90 & 0 & 0 & 0 & 2155 & $83,9 \%$ \\
\hline & PK & 0 & 564 & 0 & 0 & 0 & 0 & 564 & $100,0 \%$ \\
\hline & PL & 0 & 0 & 1627 & 101 & 0 & 0 & 1728 & $94,2 \%$ \\
\hline & $\mathrm{Q} / \mathrm{M}$ & 0 & 0 & 178 & 932 & 25 & 0 & 1135 & $82,1 \%$ \\
\hline & $\mathrm{L}$ & 0 & 0 & 0 & 0 & 1194 & 0 & 1194 & $100,0 \%$ \\
\hline & Wasser & 0 & 0 & 0 & 0 & 37 & 105 & 142 & $73,9 \%$ \\
\hline \multicolumn{2}{|c|}{ Summe } & 1808 & 821 & 1895 & 1033 & 1256 & 105 & 6918 & \\
\hline \multicolumn{2}{|c|}{$\begin{array}{l}\text { Hersteller - } \\
\text { Genauigkeit }\end{array}$} & $100,0 \%$ & $68,7 \%$ & $85,9 \%$ & $90,2 \%$ & $95,1 \%$ & $100,0 \%$ & \multicolumn{2}{|c|}{$\begin{array}{c}\text { Gesamt-Genauigkeit } \\
90,1 \% \\
\text { Kappa }: 0.87\end{array}$} \\
\hline
\end{tabular}

\subsubsection{Segmentbasierte „Maximum Likelihood“-Klassifikation}

Die Verifizierungsergebnisse der segmentbasierten „Maximum Likelihood“Klassifikation der Landsat TM-Daten sind in Tabelle 5.23 angegeben. Die Klasse „Wasser“ zeigt bei dieser Methode ebenfalls die niedrigste Nutzergenauigkeit. Fast die Hälfte der landwirtschaftlichen Flächen wurde hier als Wasser klassifiziert, obwohl diese Klasse in den Referenzflächen richtig klassifiziert wurde. Eine mögliche Ursache für die Fehlklassifikation ist, wie bereits für die pixelbasierte Klassifikation erwähnt, der unterschiedliche Feuchtigkeitsgehalt im Boden. Darüber hinaus erreichte die Klassifizierung der Larix leptorepis-Bestände lediglich eine Genauigkeit von nur 33\% aus der Sicht des Herstellers. Die meisten Flächen wurden fälschlicherweise als Q / MKlasse klassifiziert. 
Tabelle 5.23: Fehlermatrix für die segmentbasierte „Maximum Likelihood“Klassifikation der Landsat TM- Daten.

\begin{tabular}{|c|c|c|c|c|c|c|c|c|c|}
\hline \multirow{2}{*}{\multicolumn{2}{|c|}{ Klasse }} & \multicolumn{6}{|c|}{ Referrenz } & \multirow{2}{*}{ Summe } & \multirow{2}{*}{$\begin{array}{c}\text { Nutzer- } \\
\text { Genauigkeit }\end{array}$} \\
\hline & & $\mathrm{PD} / \mathrm{C}$ & PK & PL & $\mathrm{Q} / \mathrm{M}$ & $\mathrm{L}$ & Wasser & & \\
\hline \multirow{6}{*}{ 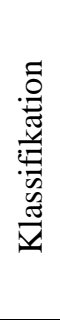 } & $\mathrm{PD} / \mathrm{C}$ & 1208 & 74 & 0 & 0 & 0 & 0 & 1282 & $94,2 \%$ \\
\hline & PK & 175 & 704 & 0 & 48 & 0 & 0 & 927 & $75,9 \%$ \\
\hline & PL & 183 & 43 & 633 & 35 & 0 & 0 & 894 & $70,8 \%$ \\
\hline & $\mathrm{Q} / \mathrm{M}$ & 0 & 0 & 1049 & 884 & 0 & 0 & 1933 & $45,7 \%$ \\
\hline & $\mathrm{L}$ & 242 & 0 & 213 & 66 & 672 & 0 & 1193 & $56,3 \%$ \\
\hline & Wasser & 0 & 0 & 0 & 0 & 584 & 105 & 689 & $15,2 \%$ \\
\hline \multicolumn{2}{|c|}{ Summe } & 1808 & 821 & 1895 & 1033 & 1256 & 105 & 6918 & \\
\hline \multicolumn{2}{|c|}{$\begin{array}{l}\text { Hersteller - } \\
\text { Genauigkeit }\end{array}$} & $66,8 \%$ & $85,7 \%$ & $33,4 \%$ & $85,6 \%$ & $53,5 \%$ & $100,0 \%$ & \multicolumn{2}{|c|}{$\begin{array}{c}\text { Gesamt-Genauigkeit } \\
60,8 \% \\
\text { Kappa }: 0.53\end{array}$} \\
\hline
\end{tabular}

\subsection{Fazit der Klassifikationsergebnisse von Landsat TM}

In Tabelle 5.24 wurden die Klassifizierungsgenauigkeiten der überprüften Methoden und die entsprechenden Kappa-Werte zusammengefaßt. Anders als bei den IKONOSDaten, bei denen die Methode der segmentbasierten „Maximum Likelihood“Klassifikation die höchste Genauigkeit lieferte, ergab hier die Methode der Mehrheitszuweisung bei der Klassifizierung der Landsat TM- die besten Ergebnisse Daten mit einer Gesamtgenauigkeit von 90 \%. Der Kappa-Wert beträgt dabei 0,87.

Tabelle 5.24: Vergleich der Genauigkeit von verschiedenen Klassifikationsmethoden für die Landsat TM-Daten.

\begin{tabular}{ccc}
\hline Methode & Gesamtgenauigkeit & Kappa \\
\hline Pixelbasierte MLK & $67 \%$ & 0,59 \\
Mehrheitszuweisung & $90 \%$ & 0,87 \\
Segmentbasierte MLK & $61 \%$ & 0,53 \\
\hline
\end{tabular}

Nach der Zusammenfassung von zwei Nicht-Wald-Klassen stieg die Gesamtgenauigkeit geringfügig auf $91 \%$ (Tabelle 5.25). In Abbildung 5.22 ist das Ergebnis der Klassifikation in Form einer Karte dargestellt. 
Tabelle 5.25: Klassifikationsergebnis der Landsat TM-Daten zur Kartierung von Waldtypen. Angaben sind prozentuale Werte aus der Sicht des Herstellers.

\begin{tabular}{c|ccccc}
\hline Klasse & PD / C & PK & PL & Q / M & Nicht-Wald \\
\hline PD / C & 100,0 & 31,3 & 4,7 & 0,0 & 0,0 \\
PK & 0,0 & 68,7 & 0,0 & 0,0 & 0,0 \\
PL & 0,0 & 0,0 & 85,9 & 9,8 & 0,0 \\
Q / M & 0,0 & 0,0 & 9,4 & 90,2 & 1,8 \\
Nicht-Wald & 0,0 & 0,0 & 0,0 & 0,0 & 98,2 \\
\hline
\end{tabular}

Gesamtgenauigkeit : $91 \%$

Eine nach Waldtypen getrennte Flächenbilanz wurde aus den Klassifikationsergebnissen der Landsat TM-Daten erstellt und ist in Tabelle 5.26 dargestellt. Der Waldflächenanteil im Untersuchungsgebiet beträgt ca. $85 \%$ der Gesamtfläche. Etwa $70 \%$ der Waldflächen waren mit Nadelbaumarten bedeckt. Die Anteile von Laubwäldern und Mischwäldern betragen zusammen ca. $30 \%$ der gesamten Waldfläche.

Tabelle 5.26: Flächenbilanz der Klassifikation der Landsat TM-Daten.

\begin{tabular}{cccc}
\hline \multirow{2}{*}{ Waldtypen } & $\begin{array}{c}\text { Flächengröße } \\
(\mathrm{ha})\end{array}$ & Gesamtfläche & Waldfläche \\
\cline { 3 - 4 } & 1724,6 & 22,5 & 26,5 \\
Pinus densiflora / & 425,4 & 5,5 & 6,5 \\
Nadel-Mischwald & 2405,0 & 31,3 & 36,9 \\
Pinus koraiensis & 1954,7 & 25,5 & 30,0 \\
Larix leptolepis & 1166,4 & 15,2 & - \\
Quercus spp. / Laub- & 7676,0 & 100 & 100 \\
Nadel Mischwald & & & \\
Nicht-Wald & & & \\
\hline Gesamt & &
\end{tabular}




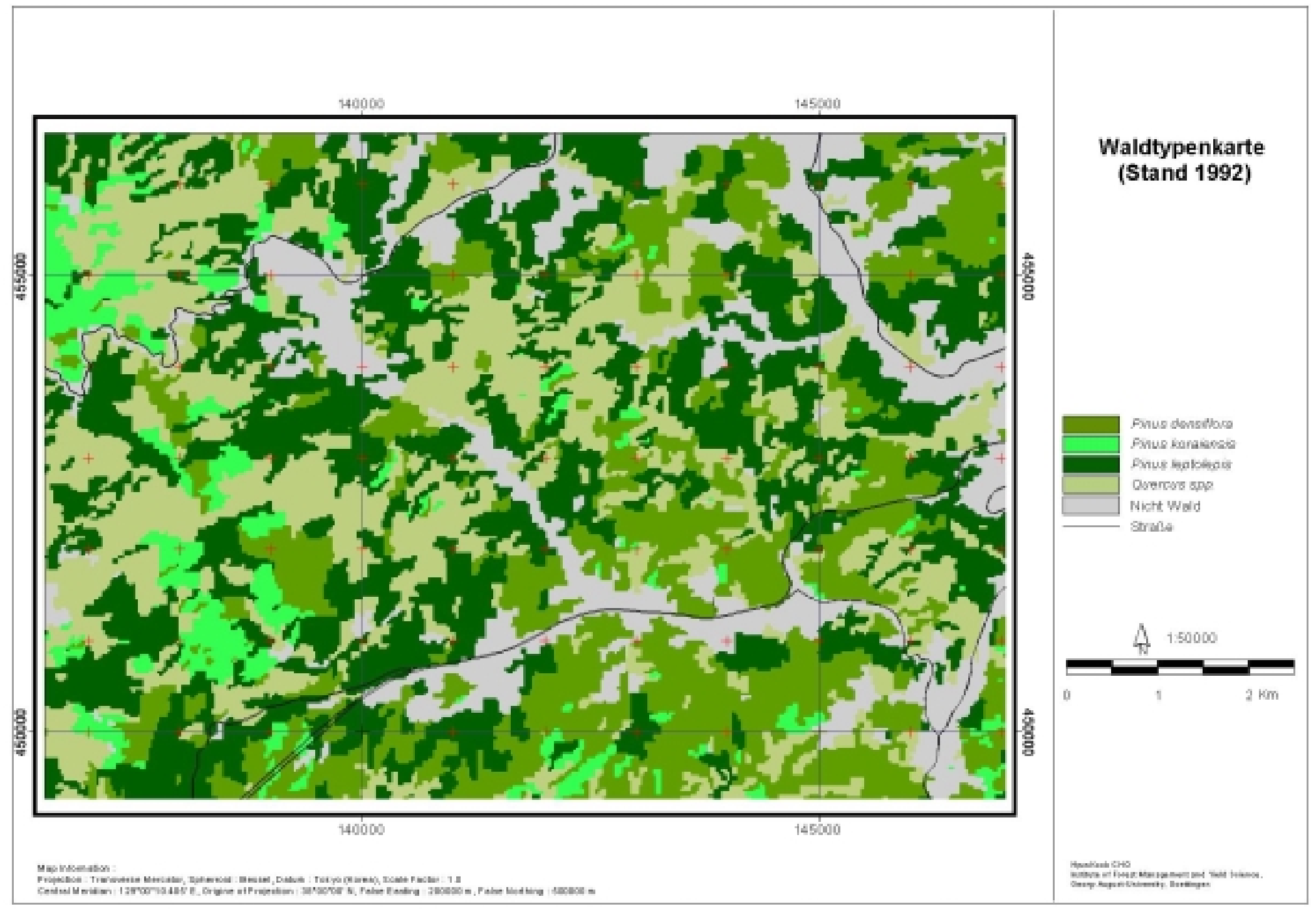

Abbildung 5.22: Waldtypenkartierung des Untersuchungsgebietes, hergeleitet aus der Klassifikation der Landsat TM-Daten aus dem Jahr 1992. 


\subsection{Erfassung der Veränderungen}

Die Erfassung der Veränderungen erfolgte durch einen Vergleich der bereits im Abschnitt 5.4 klassifizierten Satellitendaten. Hierfür wurden jeweils die Klassifikationsergebnisse mit den besten Ergebnissen, nämlich das durch die Methode der Mehrheitszuweisung klassifizierte Landsat TM-Bild vom 1992 und die segmentbasierte „Maximum Likelihood“-Klassifikation der IKONOS-Daten aus dem Jahr 2000 herangezogen.

Die beiden Datensätze mit unterschiedlichen geometrischen Auflösungen und unterschiedlichen thematischen Informationen wurden zuerst durch eine Vorverarbeitung vereinheitlicht. Die Auflösung der Landsat TM-Daten wurden durch „Resampling“ von $30 \mathrm{~m}$ auf $1 \mathrm{~m}$ transformiert. Damit konnten die detaillierten geometrischen Informationen der hochauflösenden Satellitendaten von IKONOS beibehalten werden. Die thematischen Unstimmigkeiten wurden durch Zusammenfassung der thematischen Kategorien von der detaillierten IKONOS Klassifikation bereinigt. Hierfür wurden sowohl die Klasse Nadel-Mischwald (C) und Pinus densiflora (PD) in die neue Klasse PD / C als auch die Klassen Quercus spp. (Q) und Laub-Nadel-Mischwald (M) in die Klasse Q / M umkodiert. Darüber hinaus wurden alle Nicht-Wald Klassen zusammengefaßt.

In Tabelle 5.27 sind die Flächen getrennt nach Waldtypen für die Jahr 1992 und 2000 gegenübergestellt.

Tabelle 5.27: Veränderungen der Waldflächen im Untersuchungsgebiet zwischen 1992 und 2000.

\begin{tabular}{|c|c|c|c|c|c|c|c|}
\hline \multirow[t]{2}{*}{ Jahr } & \multirow[b]{2}{*}{ Klasse } & \multicolumn{4}{|c|}{1992} & \multicolumn{2}{|r|}{ ( ha ) } \\
\hline & & $P D / C$ & PK & $\mathrm{PL}$ & $Q / M$ & Nichtwald & Summe \\
\hline \multirow{8}{*}{2000} & $P D / C$ & 520,2 & 133,1 & 405,1 & 312,3 & 63,5028 & 1434,2 \\
\hline & PK & 167,2 & 122,5 & 97,6 & 87,8 & 17,9413 & 493,0 \\
\hline & PL & 186,9 & 27,0 & 518,3 & 325,5 & 23,126 & 1080,7 \\
\hline & $\mathrm{Q} / \mathrm{M}$ & 481,4 & 112,8 & 820,6 & 880,5 & 75,4716 & 2370,8 \\
\hline & $F$ & 205,0 & 4,5 & 213,0 & 86,8 & 60,2075 & 569,5 \\
\hline & LP & 7,7 & 2,1 & 21,4 & 43,4 & 19,0206 & 93,7 \\
\hline & Nichtwald & 156,2 & 23,4 & 329,1 & 218,4 & 907,1 & 1634,2 \\
\hline & Summe & 1724,6 & 425,4 & 2405,0 & 1954,7 & 1166,4 & 7676,0 \\
\hline
\end{tabular}


Die größte Veränderung kam bei der Klasse der Larix leptolepis (PL) vor. Die Flächen dieser Klasse verringerten sich zwischen 1992 und 2000 um ca. 1300 ha. Dies ist einerseits auf Fehlklassifikationen andererseits aber auch auf methodische und geometrische (bedingt durch die Auflösung) Fehler zurückzuführen. Hierauf soll im Abschnitt 6 näher eingegangen werden

Die Flächengrößen der Wald- und Nichtwaldflächen und deren Veränderungen sind in Tabelle 5.28 angegeben. Insgesamt ist die Waldfläche im Untersuchungsgebiet im Zeitraum von 1992 bis 2000 um ca. 560 ha zurückgegangen. Die Hauptursache des Waldrückgangs ist die Einrichtung von Skigebieten und der Bau von Straßen. Die Flächenänderungen sind in Abbildung 5.23 veranschaulicht.

Tabelle 5.28: Flächengröße der Wald- bzw. Nichtwald-Klasse und deren Flächenveränderungen.

\begin{tabular}{ccc}
\hline Jahr & Wald & Nichtwald \\
\hline 1992 & 6509,7 & 1166,4 \\
2000 & 5948,2 & 1727,8 \\
Differenz & $-561,4$ & $+561,4$ \\
\hline
\end{tabular}




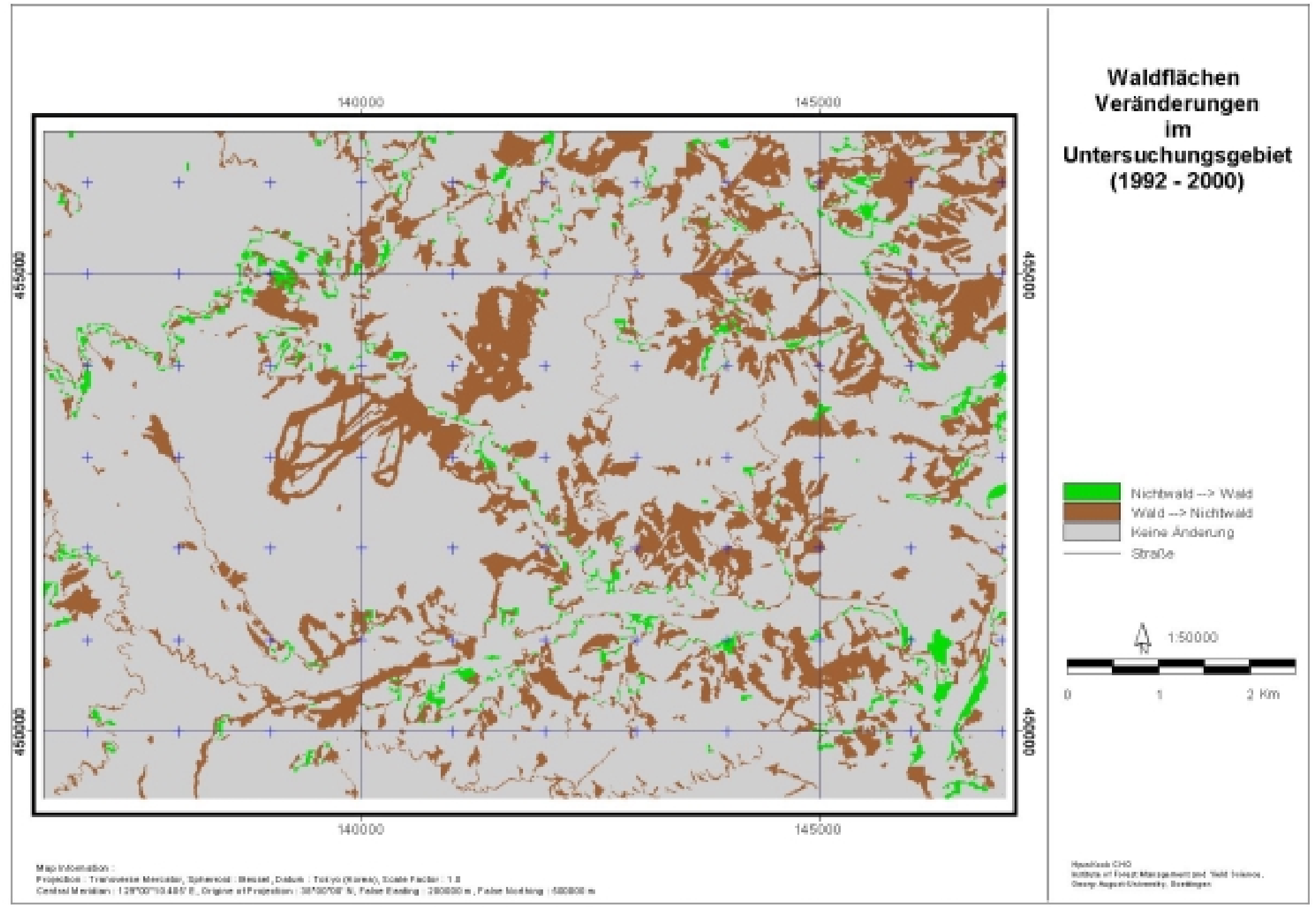

Abbildung 5.23: Veränderungen von Wald und Nichtwald im Zeitraum 1992 bis 2000 


\section{Diskussion}

\section{Georeferenzierung und geometrische Genauigkeit}

Eine genaue Georeferenzierung bildet die Grundlage für die topographische Normalisierung, die Fusion unterschiedlicher Fernerkundungsdaten und die Erfassung von Veränderungen. Darüber hinaus ist dies eine Grundvoraussetzung zur integrierten Nutzung von Daten anderer Herkunft.

Die Genauigkeit der Paßpunktmessungen aus Orthotophotos fällt in der vorliegenden Arbeit im Vergleich zu den Arbeiten von Toutin und ChEng (2001), Verfasser des Orthorektifizierungsmoduls für IKONOS-Daten, schlechter aus. Die bei ihnen erreichte Genauigkeit von 1,3m mittlere quadratische Abweichung (RMS Fehler) in X- und YRichtung resultieren wahrscheinlich aus einer höheren Paßpunktgenauigkeit und aus einem besseren Höhenmodell. Zudem berichten sie von einer Höhenamplitude im Gelände von nur ca. $60 \mathrm{~m}$.

Troz des etwas höheren Fehlers ist die Qualität der Georeferenzierung anhand der Paßpunkte und des Höhenmodells in Anbetracht der hohen Reliefenergie des Untersuchungsgebietes für forstliche Zwecke als ausreichend zu bezeichnen.

\section{Segmentierung}

Die Segmentierung bildet die Grundlage zur Durchführung einer segmentbasierten Klassifikation. Als Fehlerquellen kommen hier mehrere Faktoren in Frage.

Die Bestimmung einer optimalen Segmentgröße erfolgt heuristisch. Sie ist aus diesem Grund keine objektive sondern eine subjektive Größe und hängt von der Erfahrung des Auswerters ab.

Die Abgrenzung einzelner Segmente ist nicht immer eindeutig durchführbar, da Waldtypen fließend ineinander übergehen können und eine Undeutlichkeit der Grenzen bestehen kann.

Die Grenzziehung der Segmente erfolgt prinzipiell konform mit den im Bild abgebildeten Grenzen. Die sinnführenden bzw. semantischen Grenzen aus der 
(a)
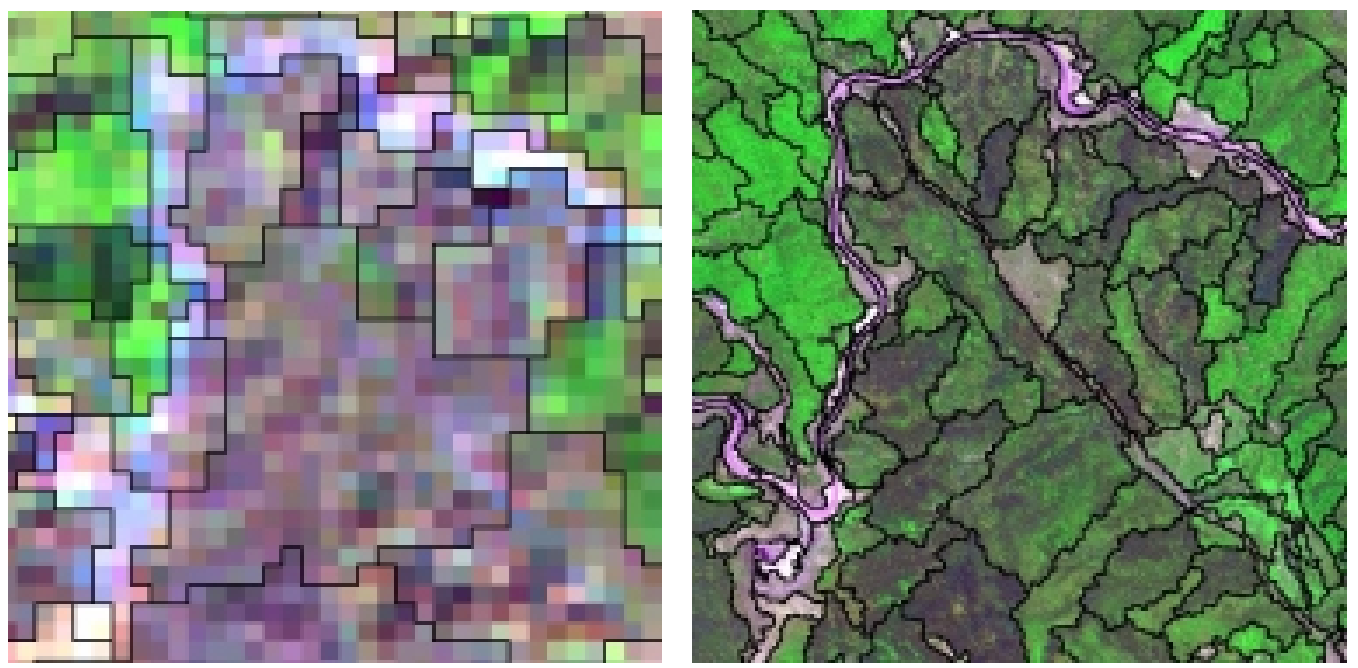

(b)
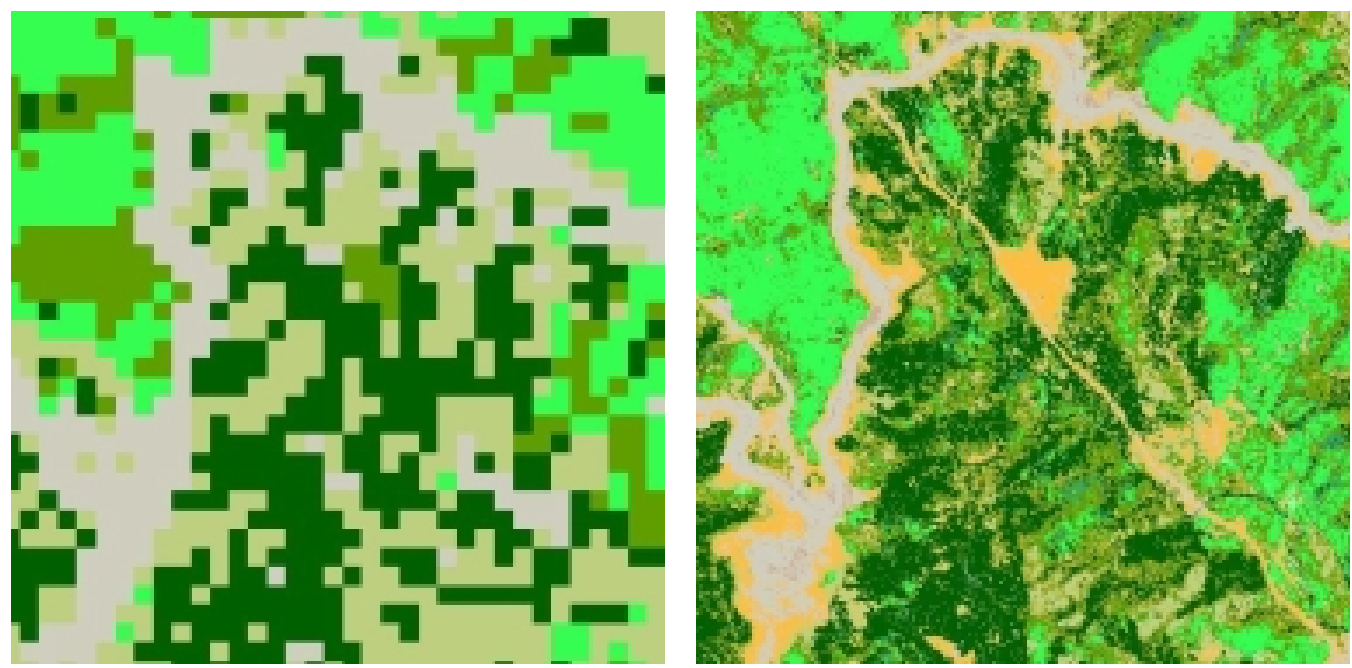

(c)
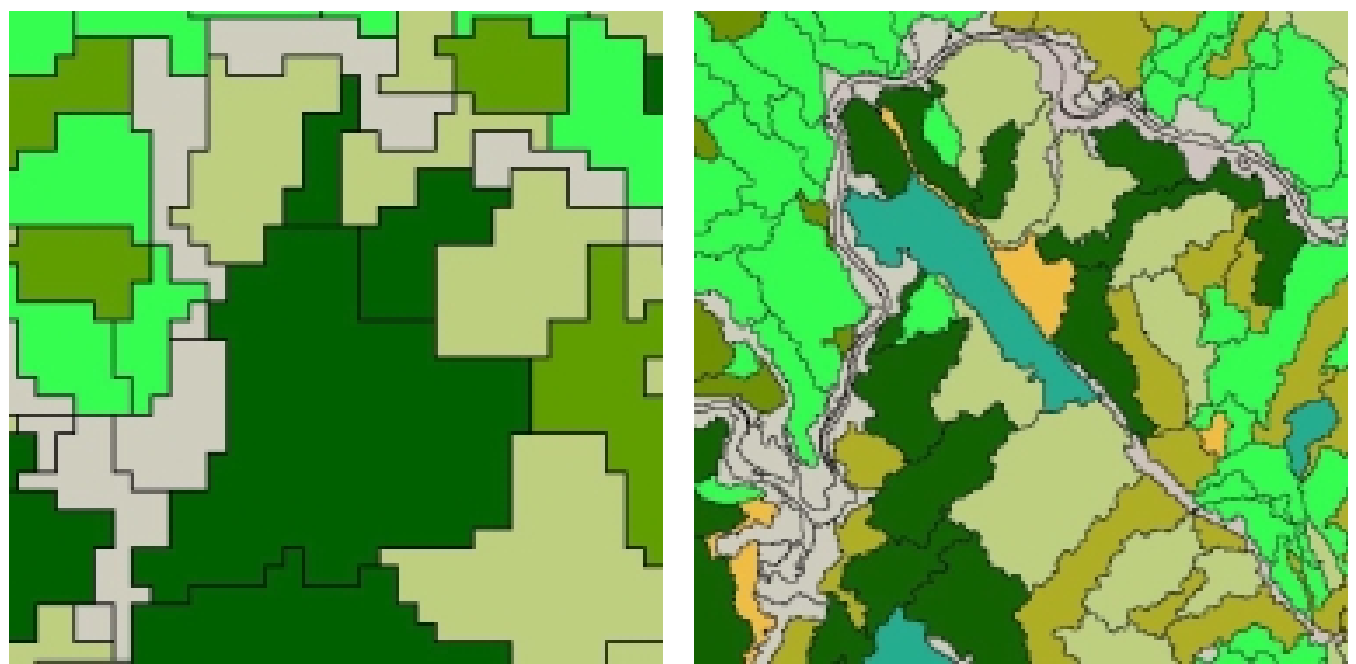

Abbildung 6.1:Vergleich der beiden Datensätze Landsat TM (links) und IKONOS (rechts) in einem kleinen Ausschnitt von ca. $1 \mathrm{~km} x 1 \mathrm{~km}$ Größe. (a) Original-Daten mit dem Grenzen der Segmente, (b) Pixelbasierte Klassifikation (c) Segmentbasierte Klassifikation. 
Segmentierung sind jedoch nicht immer mit den abgebildeten Grenzen im hochauflösenden Satellitenbild deckungsgleich. Bei Fernerkundungsdaten mit einer mittelhohen Auflösung wie z.B. bei Landsat TM-Daten ist die Feinstruktur der Klassengrenze bedingt durch die relativ grobe Auflösung des Bildes nicht abgebildet (Abbildung 6.1 (a)).

\section{Pixelbasierte „Maximum Likelihood“-Klassifikation}

Die pixelbasierte „Maximum Likelihood“-Klassifikation wurde nur anhand der spektralen Informationen des einzelne Pixels durchgeführt. Die Interpretation und Nutzung der Klassifikationsergebnisse wird dabei durch den sogenannten „Salz und Pfeffer" Effekte erschwert, ebenso wie die Integration der Ergebnisse in ein GIS. Dieser Effekt schlägt sich besonders bei der Anwendung dieser Methode auf die höchstauflösenden IKONOS-Daten nieder, weil durch die hohe Auflösung z.B. die Reflexion des Waldbodens unter Umständen auf Kleinstflächen im Kronendach pixelweise abgebildet wird (Abbildung 6.1 (b)).

Im Gegensatz dazu können bei der Landsat TM-Aufnahme verschiedene Klassen in einem Pixel abgebildet werden so daß z.B. die Reflexion eines Waldbestandes oder eines Bestandesrandes mit dem benachbarten Bestand in einem Pixel zusammengefaßt wird.

\section{Segmentbasierte Methode mit Mehrheitszuweisung}

Die höchstauflösenden Daten der IKONOS-Szene zeigen besonders im Bereich von Mischwäldern spezielle Probleme bei der Klassifizierung. Der einzelnen Pixel innerhalb eines Segmentes repräsentieren dabei entweder die Laubwaldklasse oder die Nadelwaldklasse. Bei der Mehrheitszuweisung wird dann das Segment je nach den Mehrheitsverhältnissen entweder als Laubwald oder als Nadelwald klassifiziert. Auf dieser Grundlage ist keine eindeutige Klassifikation von Mischwäldern mit der Mehrheitszuweisungsmethode durchzuführen.

Im Gegensatz dazu werden Mischwälder bei der Klassifikation von Landsat TM-Daten bedingt durch größere Pixel genauer erfaßt, da die reflektierten Spektralwerte durch die Integration über die Pixelfläche repräsentativer für den Mischbestand sind. Beim 
Vergleich der Klassifikationsergebnisse von Landsat TM-Daten mit unterschiedlichen Klassifikationsverfahren hat sich als beste Methode die Mehrheitszuweisung erwiesen.

\section{Segmentbasierte „Maximum Likelihood“-Klassifikation}

Bei der segmentbasierten „Maximum Likelihood“-Methode bildet das Segment die Grundeinheit der Klassifikation. Ein Vorteil der segmentbasierten „Maximum Likelihood“-Klassifikation ist, daß neue Merkmale nicht nur auf Basis der spektralen Information des Pixels, sondern auch auf Basis des Segmentes herangezogen werden können. Als neue Merkmale kommen z.B. der Mittelwert, die Standardabweichung, die Differenz oder das Verhältnis zwischen den Kanälen, sowie die Textur in Frage. Darüber hinaus können z.B. die Form, die Größe, der Umfang, die Kompaktheit und die „Smoothness“ sowie die hierarchischen Beziehungen der Segmente zur Klassifikation herangezogen werden.

Zur Begrenzung der zu bearbeitenden Datenmengen und aus Gründen der Plausibilität der hergeleiteten Beziehungen wurden in dieser Untersuchung nur die segmentweise hergeleiteten Mittelwerte und Standardabweichungen von Grauwerten einzelner Kanäle einbezogen.

Für die hochauflösenden Daten der IKONOS-Szene sind mit dieser Methode die besten Ergebnisse erreicht worden (s. Kap. 5.413.3). Durch die segmentweise hergeleiteten Merkmale können besser trennbaren Signaturen für die Mischwälder gewonnen worden (vergl. Tabelle 5.10 und Tabelle 5.14)

Die Klassifikation der Landsat TM-Daten führte zu keinen befriedigenden Ergebnissen bei den einzelnen Waldklassen. Hier werden bei einer relativ groben Pixelgröße von 30 x $30 \mathrm{~m}$ die in der IKONOS-Szene differenzierten spektralen Signaturen bereits zusammengefaßt. Durch die Mittelwertbildung der Pixelwerte in den einzelnen Segmenten werden die spektralen Unterschiede zwischen verschiedenen Objekten ausgeglichen.

Eine weitere methodische Entwicklung und eine Untersuchung plausibler Zusammenhänge der oben erwähnten Merkmale für die Verbesserung der segmentbasierten „Maximum Likelihood“-Methode ist erforderlich. 


\section{Integration in GIS}

Die segmentbasierte Klassifikation erzeugt keinen „Salz und Pfeffer“ Effekt, wie es bei der Anwendung der pixelbasierten Klassifikationsmethode der Fall ist ( vergl. Abbildung 6.1(a) und (b)).

Diese Eigenschaft ist für die Integration der Klassifizierungsergebnisse in ein GIS vorteilhaft.

Eine weitere Möglichkeit zur Verbesserung der Ergebnisse ist die weitere Verarbeitung der Klassifikation mit Hilfe von GIS-Operationen, wie z.B. die Auflösung bzw. Zusammenlegung von kleinflächigen Polygonen, deren Flächengröße unterhalb der einer Planungseinheit liegt, in die sie umgebenden bzw. in größere Flächen. In Abbildung 6.2 sind die Ergebnisse solcher GIS-Operationen beispielhaft dargestellt (vergl. Abbildung 6.1 (c))

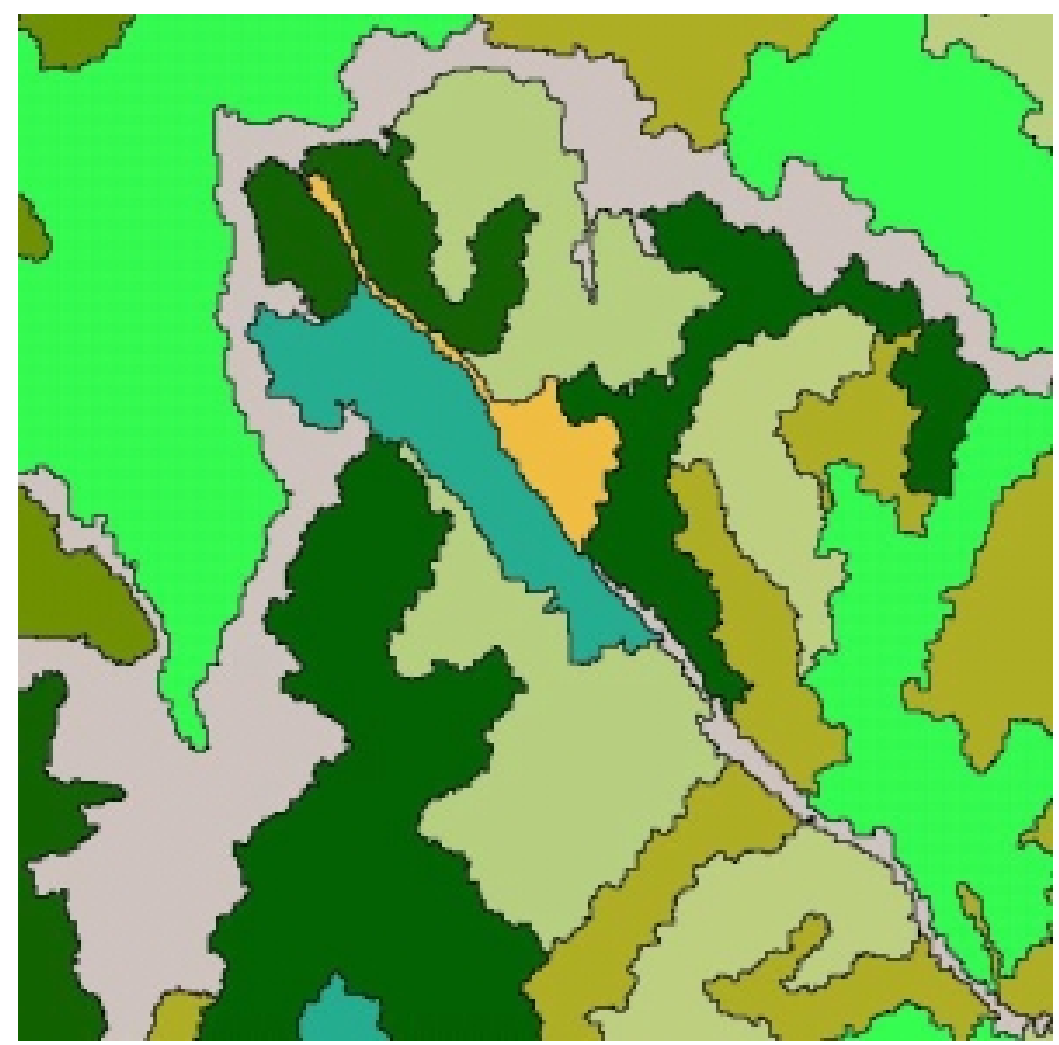

Abbildung 6.2:Beispiel einiger GIS-Operationen am Ergebnis einer segmentbasierten Klassifikation. Hier wurden zuerst alle benachbarten Segmente klassenweise zusammengelegt („Dissolve“). Danach wurden die Flächen, die kleiner als 0,5ha waren, zur meist umgebenden Klasse zugeschlagen (,Elimination“). 


\section{Erfassung der Veränderungen}

Der Zeitraum der verwendeten Satellitenbildszenen decken mit 8 Jahren ein als gut zu bezeichnendes Zeitfenster ab. Die durch die Verschiedenheit der Aufnahmesysteme erforderliche radiometrische Korrektur der Abbildungen zur Durchführung einer direkten Erfassung der Veränderung ist durch eine Vorklassifikation und Differenzbildung zwischen den Klassifikationsergebnisse umgangen worden.

Die unterschiedliche geometrische Auflösung der Satellitenbilddaten wurde durch ein „Resampling“ der Landsat TM-Daten auf $1 \mathrm{~m}$ Rasterweite angeglichen. Hierbei entstehen keine Verluste an geometrischen und thematischen Informationen.

Die hieraus resultierenden thematischen Divergenzen durch die unterschiedliche Abbildung der Landoberfläche führten $\mathrm{zu}$ einer Reduktion der Klassen. Die Zusammenlegung von mehren Klassen der IKONOS-Daten zur Anpassung an die Klassen der Landsat TM-Daten führen selbstverständlich zu einem Informationsverlust. 


\section{Zusammenfassung}

Mit der Inbetriebnahme der neuen Generation von hochauflösenden Satellitensystemen wie z.B. IKONOS wird die Anwendung von Satellitendaten in vielen Bereichen deutlich an Attraktivität gewinnen. Obwohl eine erhöhte geometrische Auflösung bessere Interpretationsmöglichkeiten bietet und den Anteil der Mischpixel reduziert, nimmt die Varianz innerhalb einer Klasse $\mathrm{zu}$, welche eine herkömmlichen pixelbasierten Klassifikation erschwert. Eine alternative Methode hierzu ist die segmentbasierte Klassifikation.

Zielsetzung dieser Arbeit ist es daher, einen Beitrag zum Einsatz von Daten der neuen Generation von hochauflösenden Satellitendaten (z.B. IKONOS) für forstliche Zwecke zu liefern. Darüber hinaus wurde die Anwendbarkeit der segmentbasierten Klassifikationsmethode für die Klassifikation sowohl einer geometrisch hoch auflösenden als auch von mittel auflösenden Satellitendaten überprüft.

Das Untersuchungsgebiet "PyeongChang" befindet sich in der Mitte der koreanischen Provinz "Kangwon-Do", die im östlichen Teil von Südkorea liegt. Das Gebiet umfasst eine Fläche von ca. $1500 \mathrm{~km}^{2}$ und liegt zwischen den geographischen Koordinaten $128^{\circ} 14^{\prime}$ und $128^{\circ} 46^{\prime}$ östliche Länge sowie zwischen $37^{\circ} 16^{\prime}$ und $37^{\circ} 49^{\prime}$ nördliche Breite.

Als Untersuchungsmaterial wurden 35 infrarot-schwarz-weiß Luftbilder vom Oktober 1992, eine Landsat 5 TM Szene vom November 1992 und ein IKONOS-GEO Szene vom April 2000 herangezogen.

Es wurde zunächst die Bereitstellung der Grundlagedaten für die Auswertung der Satellitendaten vorgestellt. Für die Herstellung des digitalen Geländemodells wird zuerst ein TIN (Triangulated Irregular Network) Model mit Hilfe von Höhenlinien sowie von Höhenwerten einiger Berggipfel erstellt, welche aus der digitalen topographischen Karte gewonnen wurden. Danach wurden zwei DGM mit einer Rastergröße von 30m bzw. $1 \mathrm{~m}$ aus dem TIN Model abgeleitet. Das DGM wurde für die Orthorektifizierung und für die topographische Normalisierung der Landsat TM Daten eingesetzt.

Für die digitale photogrammetrische Auswertung der Luftbilder wurden die Bilder mit einem einfachen Büro-Scanner und mit einer Auflösung von 300 dpi (ca. $85 \mu$ ), die bei 
einem mittleren Bildmaßstab von 1: 13000 ca. 1m auf dem Boden entspricht, digitalisiert und mit dem digitalen photogrammetrischen Auswertungssystem „Softplotter“ ausgewertet. Die Paßpunkte zur Aerotriangulation der Luftbilder wurden aus den digitalen und den analogen topographischen Karten im Maßstab 1:25000 gewonnen. Insgesamt wurden 23 Paßpunkte, davon 6 Vollpaßpunkte, 12 Lagepaßpunkte und 5 Höhenpaßpunkte für die 30 Modelle überwiegend im Bereich von Straßenkreuzungen und Brücken gesammelt. Dabei wurden 107 Verknüpfungspunkte für die Aerotriangulation herangezogen. Damit konnte eine Lagegenauigkeit von 6,5 m in X-Richtung, 8,2 m in Y-Richtung und eine Höhengenauigkeit von 10,7 m erreicht werden. Für jedes orientierte Luftbild wird ein Orthophoto erstellt. Dabei wurde eine bilineare Transformation für die Zuweisung neuer Pixelwerte herangezogen. Anschließend wurden die einzelnen Orthophotos unter „ERDAS IMAGINE“ zu einem Orthophotomosaik zusammengesetzt.

Die Luftbild-Orthophoto und -Stereoauswertung diente der Gewinnung der Paßpunkte für die Georeferenzierung der IKONOS und Landsat Daten und zusätzlich zur Gewinnung der sogenannten „Ground Truth“ als auch der Referenzdaten für die Klassifizierung der Landsat TM Satellitenaufzeichnungen.

Weiterhin wurden die multispektralen und panchromatischen Daten von IKONOS fusioniert, um damit die Vorteile beider Datensätze gleichzeitig nutzbar zu machen. Durch eine IHS Transformation wurde dies ermöglicht. Anschließend werden die geometrischen Korrekturen im Rahmen der Georeferenzierung beschrieben. Dabei liegen die Schwerpunkte auf der Erprobung verschiedener Georeferenzierungsmethoden und der Analyse ihrer Genauigkeit mit unabhängigen Kontrollpunkten für die hochauflösenden „IKONOS-Geo“ Satelliten-Daten. Mit einer Orthorektifizierung wurde ein mittlerer Lagefehler von 9,5 m und eine maximale Abweichung von 24,6 m erreicht. Der Lagefehler von 14,8 m mit CE90 (Circular Error $90 \%$ ) liegt zwischen der Genauigkeit der von „Space Imaging (SI)“ definierten Kategorien „Reference“ und „Pro“, die jedoch ca. 3 bzw. 4-fach teurer als Daten der Genauigkeitskategorie „Geo“ sind. Bei den Landsat TM-Daten ergab sich kein großer Unterschied zwischen einer polynominalen Transformation und einer Orthorektifizierung. Trotzdem zeigt die Orthorektifizierung etwas bessere Resultate. 
Darüber hinaus wird die topographische Normalisierung der Landsat TM-Daten für die Eliminierung bzw. Minimierung topographiebedingter Einflüsse bei der Klassifizierung dargestellt. Die topographische Normalisierung wurde mit der Minnaert-Konstante Methode durchgeführt. Durch eine visuelle Betrachtung konnte dabei festgestellt werden, daß die topographiebedingten Beleuchtungsunterschiede in der korrigierten Szene weitgehend normalisiert wurden.

Die Klassifikation der Satellitendaten wurde sowohl durch die herkömmliche pixelbasierte Methode als auch durch die segmentbasierte Methode durchgeführt. Für die pixelbasierte Klassifikation wurde die „Maximum Likelihood“ Methode herangezogen. Als segmentbasierte Klassifikation wurden die Methode der Mehrheitszuweisung und die „Maximum Likelihood“ Klassifikation auf Segmentbasis verwendet. Anschließend wurden die Ergebnisse der Methoden anhand der Genauigkeit der Klassifikation miteinander verglichen und überprüft. Unter den verwendeten Methoden wurde die Methode „segmentbasierte Maximum Likelihood Klassifikation“ mit einer Gesamtgenauigkeit von ca. $82 \%$ als best geeignete Methode zur Klassifikation der IKONOS-Daten ermittelt. Für die Landsat TM-Daten ergab sich die Methode der Mehrheitszuweisung als die am besten geeignete Methode mit einer Gesamtgenauigkeit von 90 \%. Der Kappawert beträgt dabei 0,87. Die Ergebnisse der Klassifikation wurden in Form einer Karte dargestellt.

Aufgrund der Klassifikation der beiden Satellitenszenen mit unterschiedlichem Aufnahmezeitpunkt konnte die Waldveränderung erfaßt werden. Da die beiden Klassifikationsergebnisse unterschiedliche geometrische und thematische Informationen beinhalten, wurden zuerst die beiden Datensätze durch eine Vorverarbeitung einheitlich gemacht. Anschließend konnten die Flächenveränderungen der zusammengefaßten Waldklasse sowie der Nichtwaldklasse angegeben werden. Insgesamt ist im Zeitraum von 1992 bis 2000 die Waldfläche um ca. 560 ha zurückgegangen. 


\section{Literatur}

AKÇA, A., Pahl, A., Feldkötter, C. (1996) Fernerkundung, terrestrische Aufnahmen und Geoinformationssysteme zum Monitoring subtropischer und tropischer Wälder, Forstarchiv, 67(2):53-59.

AкÇA, A. (2001) Waldinventur, Sauerländer's Verlag.

BAATZ, M. und SCHÄPE, A. (2000) Multiresolution Segmentation-an optimization approach for high quality multi-scale image segmentation, Angewandte Geographische Informationsverarbeitung XII, STROBL, J. et al. (Hrsg.) Beiträge zum AGIT-Symposium Salzburg 2000, Karlsruhe, Herbert Wichmann Verlag, S. 12-23.

BÄHR und VÖGTLE (1991) Digitale Bildverarbeitung, Wichmann Verlag.

BÄHR und WIESEL (1991) Cost-Benefit Analysis of Digital Orthophoto Technology, Digital Photogrammetric Systems, EBNER, FrITSCH und HEIPKE (Eds.) Wichmann Verlag.

BAltsavias, E.P. und BILL, R. (1994) Scanners-a Survey of Current Technology and Future Needs, IAPRS, 30(1):130-143.

Baltsavias, E.P. und Patias P. (1995) On the Use of DTP Scanners for Cartographic Applications, Proc.17. International Cartographic Conference, 3.-9. September, Barcelona, Spain, S. 1179-1189.

BAltsavias, E.P. und WAegli B. (1996) Quality Analysis and Calibration of DTP Scanners, IAPRS, 31(1):13-19.

BAltsavias, E.P. (1999) On the Performance of Photogrammetric Scanners. Photogrammetric Week '99, D. FrITSCH and R. SPILLER (Eds.) Wichmann Verlag, Heidelberg, S. 155-173.

BEON, MU-SuP (1997) Verjuengungsoekologische Untersuchungen in Kiefern (Pinusdensiflora)-Eichen-(Quercus-spp.)-Mischbestaenden Suedkoreas, Diss. Univ. Göttingen.

Beucher, S. und Meyer, F. (1993) The Morphological Approach to Image Segmentation: The Watershed Transformation, DoUGHERTY, E. R. (Hg.)

CARPer, J. W., T. M. Lillesand und Kiefer, R. W. (1990) The use of intensity-huesaturation transforms for merging SPOT panchromatic and multispectral image data, Photogrammetric Engineering \& Remote Sensing, 56:459-467. 
Chaudhuri, V. und SARKar, N. (1995) Texture Segmentation Using Fractal Dimension, IEEE Trans. Pattern Analysis and Machine Intelligence, PAMI17:72-77.

CHEn, X. W. (2002) Using remote sensing and GIS to analyse land cover change and its impacts on regional sustainable development, International Journal of Remote Sensing, 23(1) 107-124.

Cho, HyunKook, Akca, A. und Lee, Woo-Kyun (1999) Correction of Topographic Effects on Landsat TM Imagery for Forest Type Classification in Mountainous Area, Korean Journal of Forest Measurements 2(1):50-59.

Chung, Dong-Jun (1996) Konkurrenzverhaeltnisse und Struktur eines natuerlichen Pinus densiflora-Quercus variabilis-Mischwaldes in Korea, Diss. Univ. Göttingen.

Civco, D. L. (1989) Topographic Normalization of Landsat Thematic Mapper Digital Imagery, Photogrammetric Engineering \& Remote Sensing, 55:1303-1309.

ColBY, J. D. (1991) Topographic Normalization in Rugged Terrain, Photogrammetric Engineering \& Remote Sensing, 57:531-537.

Collins, J. B. und Woodcock, C. E. (1996) An assessment of several linear change detection techniques for mapping forest mortality using multitemporal landsat TM data, Remote Sensing of Environment, 56(1) 66-77.

Congalton, R. G., Oderwald, R. G. und Mead, R. A. (1983) Assessing Landsat Classification Accuracy Using Discrete Multivariate-Analysis Statistical Techniques, Photogrammetric Engineering \& Remote Sensing, 49:1671-1678.

Congalton, R. G. und Mead, R. A. (1983) A Quantitative Method to Test for Consistency and Correctness in Photointerpretation, Photogrammetric Engineering \& Remote Sensing, 49:69-74.

Congalton, R. G. (1988) A Comparison of Sampling Schemes Used in Generating Error Matrices for Assessing the Accuracy of Maps Generated from Remotely Sensed Data, Photogrammetric Engineering \& Remote Sensing, 54(5) 593600 .

Congalton, R. G. (1991) A Review of Assessing the Accuracy of Classifications of Remotely Sensed Data, Remote Sensing of Environment, 37(1) 35-46.

Congalton, R. G., Balogh, M., Bell, C., Green, K., Milliken, J. A. und Ottman, R. (1998) Mapping and Monitoring Agricultural Crops and Other Land Cover in the Lower Colorado River Basin, Photogrammetric Engineering \& Remote Sensing, 64:1107-1113. 
Cook, R., McConnell, I., Stewart, D. und Oliver, C. (1994) MUM (Merge Using Moments) Segmentation for SAR Images, Proc. SPIE 2316, S. 92-103.

Cooley, J. H. und Cooley, T. (1999) Segmentation and Discrimination of Structural and Spectral Information Using Multi-Layered Pulse Couple Neutral Networks, Proc. IEEE 1999 International Geoscience and Remote Sensing Symposium, 28 June-2 July 1999, Hamburg, Germany, Piscataway, CD-Rom.

CopPIN, P., NACKAERTS, K. et al. (2001) Operational monitoring of green biomass change for forest management, Photogrammetric Engineering \& Remote Sensing, 67(5) 603-611.

Darvishsefat, A. A. (1995) Und-Fusion von Multisensoralen Satellitendaten Einsatz zur Erfassung von Waldinventuren, Universität von Zürich, Abteilung von Geographie, Remote Sensing Series Vol. 24.

DefiniEns Imaging (2000) User Guide der Software eCognition, München.

Dulyakarn, P., Rangsanseri, Y. und Thitimajshima, P. (1999) Segmentation of Multispectral Images Based on Multithresholding, Proc. 2nd International Symposium on Operationalization of Remote Sensing, 16-20 August 1999, Enschede, Netherlands, CD-Rom.

ERDAS, (1999) ERDAS Field Guide, 5th Edition, S. 698.

FARAG, A. A. (1992) Edge-based Segmentation, Remote Sensing Reviews, 6:95-122.

Felzenszwalb, P. F. und Huttenlocher, D. P. (1998) Image Segmentation Using Local Variation, Proc. IEEE Conference on Computer Vision and Pattern Recognition, June 1998, Santa Barbara, USA, S. 98-104.

FGDC, (1998) Geospatial Positioning Accuracy Standards, Part3: National Standard for Spatial Data Accuracy, FGDC-STD-007.3-1998, Federal Geographic Data Committee, Reston, Virginia, USA, http://fgdc.er.usgs.gov/fgdc.html.

Fjortoft, R., Lopees, A., Marthon, P. und Cubero-Castan, E. (1998) An Optimal Multiedge Detector for SAR Image Segmentation, Trans. Geoscience and Remote Sensing, 36(3) S. 793-802.

Frery, A. C., Lucca, E. V. D., Freitas, C. d. C. und Santeanna, S. J. S. (1999) SAR Segmentation Algorithms: a Quantitative Assessment, Proc. IEEE 1999 International Geoscience and Remote Sensing Symposium, 28 June-2 July 1999, Hamburg, Germany, Piscataway, CD-Rom.

García-Haro, F. J., GILABERt, M. A. et al. (2001) Monitoring fire-affected areas using Thematic Mapper data. International Journal of Remote Sensing, 22:533 
Gillespie, A. R., Kahle, A. B. und Walker, R. E. (1986) Color Enhancement of Highly Correlated Images .1. Decorrelation and Hsi Contrast Stretches, Remote Sensing of Environment, 20:209-235.

GoRTE, B. (1989) Probabilistic Segmentation of Remotely Sensed Images, ITC Publication Series No. 63. Enschede.

Greenwalt, C.R. und Shultz, M.E. (1962) Principles of error theory and cartographic applications; Technical Report No. 96,. U.S. Air Force Aeronautical Chart and Information Center. St. Louis, Missouri.

Gregory, W. P., und Compagna, D. J. (1990) Hyperspherical direction cosine transformation for separation of spectral and illumination information in digital scanner data. Journal of Photogrammetric Engineering and Remote Sensing, 56, 475-479.

Hame, T., HeIler, I. et al. (1998) An unsupervised change detection and recognition system for forestry, International Journal of Remote Sensing, 19(6) 10791099.

Haralick, R.M., Shanmugan K. und Dinstein, I. (1973) Texture features for image classification, IEEE Systems Man and Cybernetics, 3(6), 610-621.

Haralick, R. M. und Shapiro, L. G. (1992) Computer and Robot Vision. Volume 1. AddisonWesley, Reading.

HILDEBRANDT, G. (1996) Fernerkundung und Luftbildmessung für Forstwirtschaft, Vegetationskartierung und Landschaftsökologie. Wichmann Verlag, Heidelberg.

Hofmann, T., Puzicha, J. und Buhmann, J. M. (1998) Unsupervised Texture Segmentation in a Deterministic Annealing Framework. IEEE Trans. Pattern Analysis und Machine Intelligence, PAMI-20(8):803-818.

Huss, J. (1984) Luftbildmessung und Fernerkundung in der Forstwirtschaft. Wichmann Verlag, Karlsruhe.

Jain, A. K. und Farrokhnia, F. (1991) Unsupervised Texture Segmentation Using Gabor Filters, Pattern Recognition, 24(12) S. 1167-1186.

Janssen, L. L. F. und van der Wel, F. J. M. (1994) Accuracy Assessment of Satellite Derived Land-Cover Data: A Review. Photogrammetric Engineering \& Remote Sensing, 60(4) 419-426.

JENSEN, J.R. (1996) Introductory digital image processing - a remote sensing perspective. Prentice Hall. Upper Saddle River, 318 S. 
Jo, Myung-Hee, Seong-Ryul BaeK, Mal-Sook Kim, Yun-Won Jo und Si-Young LEE (2000) The Construction of Forest Fire Monitoring System using Internet GIS and Satellite images, Proceedings of international symposium on Remote Sensing, November 1-3, 2000,Kyung-Ju, Korea, 61-64.

Jones, A.R., SetTle, J.J. und Wyatt, B.K. (1988) Use of Digital Terrain Data in the Interpretation of Spot-1 Hrv Multispectral Imagery. International Journal of Remote Sensing, 9(4):669-682.

Kartikeyan, B., Sarkar, A. und Majumder, K. L. (1998) A Segmentation Approach to Classification of Remote Sensing Imagery. International Journal of Remote Sensing, 19(9) S. 1697-1709.

KELLENBERGER, TOBIAS W. (1996) Erfassung der Waldfläche in der Schweiz mit multispektralen Satellitenbilddaten-Grundlagen, Methodenentwicklung und Anwendung, Diss. ETH Zürich.

Keller, J. M. und Chen, S. (1989) Texture Description and Segmentation Through Fractal Geometry, Computer Vision, Graphics, And Image Processing 45, S. 150-166.

KFS: Korea Forest Service (2001) Management of Forest Resources Using Remote Sensing, S. 260.

KFRI: Korea Forest Research Institute (1996) Manual of National Forest Inventory

KIM, CHUL-MIN (1991) Studies on Analysis of Forest Distribution and Characteristics in Gumo-san Provincial Park Using Landsat Thematic Mapper Data and Digital Terrain Model, Dissertation, Seoul National University, Korea.

Kim, Joon Bum, Jo, Myung Hee OH, Jeong Soo Lee, Kwang-Jae Park, Sung-Joong und UM, HyANG-HeE (2001) Temporal and spatial correlation analysis of Bursaphelenchus xylophililus damaged area and meteorological factors using GIS and satellite images, Proceeding of 3rd conference on agricultural and forest meteorology, S.49-52,.

KIM, SANG-WoOK und CHONG-HwA PARK (2000) Spatio-temporal change detection of forest patches of North Korea between 1979 and 1998, Proceedings of international symposium on Remote Sensing, , November 1-3, 2000,Kyung$\mathrm{Ju}$, Korea, 276-281.

KING, D. (1997) Fast Algorithms for Simplifying Large Spatial Data. http://www.cc.gatech.edu/-kingd/6155p1.html (Datum 05.Jul.2002)

KMA: Korea Meteorogical Administration (2001) Annual Meteorological Statistic. 
KraUs, K. (1990) Fernerkundung Band 2, Auswertung photographischer und digitaler Bilder, Bonn, Ferd. Dümmlers Verlag.

Kraus, K. (1996) Photogrammetrie. Band 2. Verfeinerte Methoden und Anwen-dungen. Dümmler Verlag, Bonn.

KRZYSTEK, P. und WILD D. (1992) Experimental Accuracy Analysis of Automatically Measured Digital Terrain Models. Robust Computer Vision, Foerstner/Ruwiedel (Eds.) Wichmann Verlag, Karsruhe, Germany, S. 372 390.

KunZ, D., VöGTLE, T. und Schilling, K.-J. (1998) Integrierte Verarbeitung von Satellitenbild und vektorieller Karteninformation, BÄHR, H.-P. und T. VÖGTLE (Hg.)

Kurgöllüs, F. und SANKUR, B. (1999) Image Segmentation Based on Multi-Scan Constraint Satisfaction Neural Network, Pattern Recognition Letters, 20:1551-1563.

Leblon, B., AleXAnder, M. et al. (2001) Monitoring fire danger of northern boreal forests with NOAA-AVHRR NDVI images. International Journal of Remote Sensing, 22(14):2839-2846.

LeE, Kyu-Sung und JeOng-Hyun Kim (2000) Change Analysis of Forest Area and Canopy Conditions in, Kaesung, North Korea Using Landsat, SPOT and KOMPSAT Data, Journal of the Korean Society of Remote Sensing, 16(4):327-338.

Lee, Seung-Ho, Song-Hak Choung und Jang-Ho Song, (1998) Forest resources inventory of North Korean using satellite remote sensing data, FRI journal of forest science, 58:1-13.

LeE Seung-HO, CheOl-Min Kim und Dong-HA OH (1999) Analysis of Forest Types and Stand Structures over Korean Peninsula Using NOAA/AVHRR Data, Proceedings of International Symposium on Remote Sensing, November 3-5, 1999, Kyangnung, Korea, S. 386-389.

LI, S. Z. (1995) Markov Random Field Modeling in Computer Vision. Springer, Tokyo.

LiU, F. und PickARD, R. W. (1996) Periodicity, Directionality, and Randomness: Wold Features for Image Modeling and Retrieval, IEEE Trans. Pattern Analysis and Machine Intelligence, PAMI-18(7):722-733.

Ma, Ho-Seop, Young-Gwan Chung Su-Young Jung und Dong-Wook Choi (1999) Evaluation of Damaged Stand Volume in Burned Area of Mt. Weol-A using Remotely Sensed Data, Journal of KAGIS(The Korean Association of Geographic Information Studies), 2(2):79-86. 
MAS, J.-F. (1999) Monitoring land-cover changes: a comparison of change detection techniques. International Journal of Remote Sensing, 20:139-152.

MATHER, P.M. (1999) Computer Processing of Remotely-Sensed Images, John Wiley \& Sons.

McKeown, D. M. (1988) Building Knowledge-Based Systems for Detecting ManMade Structures from Remotely Sensed Imagery. Philosophical Trans. Royal Society of London, S. 423-435.

MeIER, H.R. (1984) Progress by Forward Motion Compensation in Cameras. Proc. of 15th ISPRS Congress, 25(1):194-203.

MetTernicht, G. (1999) Change detection assessment using fuzzy sets and remotely sensed data: an application of topographic map revision. Isprs Journal of Photogrammetry and Remote Sensing, 54(4):221-233.

Meyer, F. und Beucher, S. (1990) Morphological Segmentation, Journal of Visual Communication and Image Representation, 1(1):21-46.

MiLIARESIS, G. C. (1999) Automated Segmentation of Alluvial Fans to Regions of High to Intermediate Flood Hazards from Landsat Thematic Mapper Imagery, 2nd International Symposium on Operationalization of Remote Sensing, 16-20 August 1999, Enschede, The Netherlands. Enschede, CD-Rom.

Muchoney, D. M. und HAACK, B. N. (1994) Change Detection for Monitoring Forest Defoliation. Photogrammetric Engineering \& Remote Sensing, 60(10):12431251.

MÜSIGMANN, U. (1992) Bewertung inhomogener fraktaler Strukturen und Skalenanalyse von Texturen. Springer, Berlin.

NIEMEYER, I. (2001) Satelliten und nukleare Kontrolle. Änderungsdetektion und objektorientierte, wissensbasierte Klassifikation von Multispektralaufnahmen zur Unterstützung der nuklearen Verifikation, Schriften des Forschungszentrums Jülich, Reihe Umwelt/Environment, Band 28. Jülich.

Nirala, M. L. und Venkatachalam, G. (2000) Technical Note: Hyperspherical Direction Cosine Transformation of Remotely Sensed Data for Separation of Topographic Expression of Land Use Classification, International Journal of Remote Sensing , 21:2203-2212.

Nunez, J., Otazu, X. Fors, Prades, O. A. Pala, V. und Arbiol R. (1999) Multiresolution-Based Image Fusion with Additive Wavelet Decomposition, Ieee Transactions on Geoscience and Remote Sensing, 37:1204-1211. 
OH, Dong Ha Und KaP DuK KIM (1990) A Study on the Changes of Land Use and Stand Volume around Mt. Kuem-O using Aerial Photographs, 79(4)388-397.

OH, Dong-HA (1998) Spatial Analysis and Change Detection of Pinus densiflora Stands Damaged by Pine Gall Midge using Landsat TM and Digital Map Data, Dissertation, Seoul National University, Korea.

Pal, S. K., A. Ghosh und Shankar, B. U. (2000) Segmentation of Remotely Sensed Images with Fuzzy Thresholding, and Quantitative Evaluation, International Journal of Remote Sensing, 21:2269-2300.

Peng, G., und PhILIP, J. H. (1990) An assessment of some factors in uencing multispectral land cover classi. cation. Journal of Photogrammetric Engineering and Remote Sensing, 56:597-603.

Pesari, M. und Kanellopoulos, I. (1999) Detection of Urban Features Using Morphological Based Segmentation and Very High Resolution Remotely Sensed Data, Kanellopoulos, I., Wilkinson, G. G. und T. Moons (Hg.)

Pohl, C. und van Genderen, J. L. (1998) Multisensor Image Fusion in Remote Sensing: Concepts, Methods and Applications, International Journal of Remote Sensing, 19:823-854.

PRÖBSTING, T. (1996) Luftbildgestützte Verfahren für die Forstwirtschaft: Bedarf, Anwendungsbereiche, Auswertungssysteme, Diss. Univ. Göttingen.

Puzicha, J. und Buhmann, J. M. (1999) Multiscale Annealing for Real-Time Unsupervised Texture Segmentation, Computer Vision and Image Understanding, 76(3) S. 213-230.

Puzicha, J., Hofmann, T. und Buhmann, J. M. (1999) Histogramm Clustering for Unsupervised Segmentation and Image Retrieval. Pattern Recogniton Letters, 20(9):899-909.

RADBERGER, R. (2001) Monitoring der Verstädterung im Großraum Istanbul mit den Methoden der Fernerkundung und der Versuch einer räumlich-statistischen Modellierung, Dissertation Universität Göttingen.

Rehrauer, H., SeIDEl, K. und Datcu, M. (1998) Multiscale Image Segmentation with a Dynamic Label Tree, Proc. IEEE 1998 International Geoscience and Remote Sensing Symposium, 6-10 July 1998, Seattle, USA. Piscataway, CDRom.

RichARDS John A. (2000) Remote Sensing Digital Image Analysis : An Introduction, Springer Verlag. 
Sagerer, G. und Niemann H. (1997) Semantic Networks for Understanding Scenes. Advances in Computer Vision and Machine Intelligence, Plenum Press, New York.

SCHNÖRR, C. (1997) Variationsansätze zur Bildsegmentation und Merkmalsextraktion. Habilitationsschrift, Universität Hamburg, Fachbereich Informatik, Arbeitskreis Kognitive Systeme, Hamburg.

SchowengerdT, R. A., (1997) Remote Sensing : Models and Methods for Image Processing, Academic Press.

Shandley, J., Franklin, J. und White, T. (1996) Testing the Woodcock-Harward Image Segmentation Algorithm in an Area of Southern California Chaparral and Woodland Vegetation, International Journal of Remote Sensing, 17(5) S. 983-1004.

ShetTigarA, V. K. (1992) A generalized component substitution technique for spatial enhancement of multispectral images using a higher resolution data set, Photogrammetric Engineering \& Remote Sensing, 58:561-567.

SingH, A. (1989) Digital Change Detection Techniques Using Remotely-Sensed Data, International Journal of Remote Sensing, 10:989-1003.

Skalet, C.D., LeE G.Y.G., und LADNER, L.J. (1992) Implementation of Softcopy Photogrammetric Workstations at the U.S. Geological Survey, Photogrammetric Engineering \& Remote Sensing., 58(1):57 - 63.

Smith, J. H., J. D. WiCKHAM, et al. (2002) Impacts of patch size and land-cover heterogeneity on thematic image classification accuracy. Photogrammetric Engineering \& Remote Sensing, 68(1) 65-70.

SoILlE, P. (1998) Morphologische Bildverarbeitung. Grundlagen, Methoden, Anwendungen. Springer, Berlin.

Song, C., Woodcock,C. E. et al. (2001) Classification and Change Detection Using Landsat TM Data When and How to Correct Atmospheric Effects? Remote Sensing of Environment, 75(2) 230-244.

Sonka, M., Hlavac, V. und Boyle, R. (1998) Image Processing, Analysis, and Machine Vision. 2. Auflage. PWS Publishing, Pacific Grove.

StehMAN, S. V. (1996) Estimating the kappa coefficient and its variance under stratified random sampling. Photogrammetric Engineering \& Remote Sensing, 62(4) 401-407. 
Stehman, S. V. und CzAPlewski, R. L. (1998) Design and analysis for thematic map accuracy assessment: Fundamental principles. Remote Sensing of Environment, 64(3) 331-344.

SteHMAN, S. V. (1999) Basic probability sampling designs for thematic map accuracy assessment. International Journal of Remote Sensing, 20(12) 2423-2441.

Story, M. und Congalton, R. G. (1986) Accuracy Assessment: A User's Perspective. Photogrammetric Engineering \& Remote Sensing, 52(3):397-399.

SwaIN P.H. und DAvis, S.M. (1978) Remote Sensing: The Quantitative Approach.

Teillet, P. M. Guinfin B. und Goodenough, D. G. (1982) On the Slope-Aspect Correction of Multispectral Scanner Data, Canadian Journal of Remote Sensing, 8:84-106.

TeILlET, P. M. (1986) Image Correction for Radiometric Effects in Remote Sensing, International Journal of Remote Sensing, 7:1637-1651.

Thitimajshima, P. und Chumsamrong, W. (1999) SAR Segmentation Using Multiresolution Fuzzy Clustering, Proc. 2nd International Symposium on Operationalization of Remote Sensing, 16-20 August 1999, Enschede, The Netherlands. Enschede, CD-Rom.

Tilton, J. C. (1998) Image Segmentation by Region Growing and Spectral Clustering with a Natural Convergence Criterion, Proc. IEEE 1998 International Geoscience and Remote Sensing Symposium, 6-10 July 1998, Seattle, USA. Piscataway, CD-Rom.

Tilton, J. C. (1996) Hybrid Image Segmentation for Earth Remote Sensing Data Analysis, Proc. IEEE 1996 International Geoscience and Remote Sensing Symposium, 27-31 May 1996, Lincoln, USA. Piscataway, CD-Rom.

TÖNJES, R. (1999) Wissensbasierte Interpretation und 3D-Rekonstruktion von Landschaftsszenen aus Luftbildern. Fortschritt-Berichte VDI, Reihe 10, Nr. 575. VDI-Verlag, Düsseldorf.

Toutin, T (1985) Analyse mathématique des capacités stéréoscopiques du système SPOT. Thèse de Docteuringénieur, Ecole Nationale des Sciences Géodésiques, Paris, France.

Toutin, T., Carbonneau, Y. und Stlaurent, L. (1992) An Integrated Method to Rectify Airborne Radar Imagery Using Dem, Photogrammetric Engineering and Remote Sensing, 58:417-422,.

Toutin, T. und Carbonneau, Y. (1992) Mos and Seasat Image Geometric Corrections, Ieee Transactions on Geoscience and Remote Sensing, 30:603-609,. 
Toutin, T. (1995) Integration of Multisource Data-Comparison of Geometric and Radiometric Methods, International Journal of Remote Sensing, 16:27952811, .

Toutin, T. und Cheng, P. (2000) Demystification of Ikonos!, EOM, 9:17-21,.

U.S. GeOlOGICAL SuRVEY (1990) The spatial data transfer standard, Draft, January.

Wang, L. und LIU, J. (1999) Texture Classification Using Multiresolution Markov Random Field Models, Pattern Recogniton Letters, 20(2) 171-182.

Won, KAng-Yung und Im, Jung-Ho ( 2001) Fire Severity Mapping Using a Single Post-Fire Landsat 7 ETM+ Imagery, Korean Journal of Remote Sensing, 17(1):85-97.

Woodcock, C. E. und Harward, V. J. (1992) Nested-Hierarchical Scene Models and Image Segmentation, International Journal of Remote Sensing, 13(16) 31673187.

Woodham, R. J. und Gray, M. H. (1987) An Analytic Method for Radiometric Correction of Satellite Multispectral Scanner Data. Ieee Transactions on Geoscience and Remote Sensing, 25(3):258-271.

Xuan, M. O. und AdAH T. (1996) Task-Specific Segmentation of Remote Sensing Images, Proc. IEEE 1996 International Geoscience and Remote Sensing Symposium, 27-31 May 1996, Lincoln, USA. Piscataway, CD-Rom.

Yun, Jong Wha und CHA, Du Song (1995) Studies on the estimation of Korean pine(pinus koraiensis) stand structure by aerial photographs, Journal of Korean forestry society, 84(4):.437-446.

ZERDA, H. R. (1998) Monitoring der Vegetations-und Landnutzungsveränderungen durch Brandrodung und Übernutzung im Trocken-Chaco Argentiniens mit Satellitenfernerkundung und GIS. Dissertation Universität Göttingen.

ZhU, Z. L., YANG, L. M. et al. (2000) Accuracy assessment for the US Geological Survey Regional Land-Cover Mapping Program: New York and New Jersey region, Photogrammetric Engineering \& Remote Sensing, 66(12):1425-1435. 


\section{Anhang}

\subsection{IKONOS Metadata}

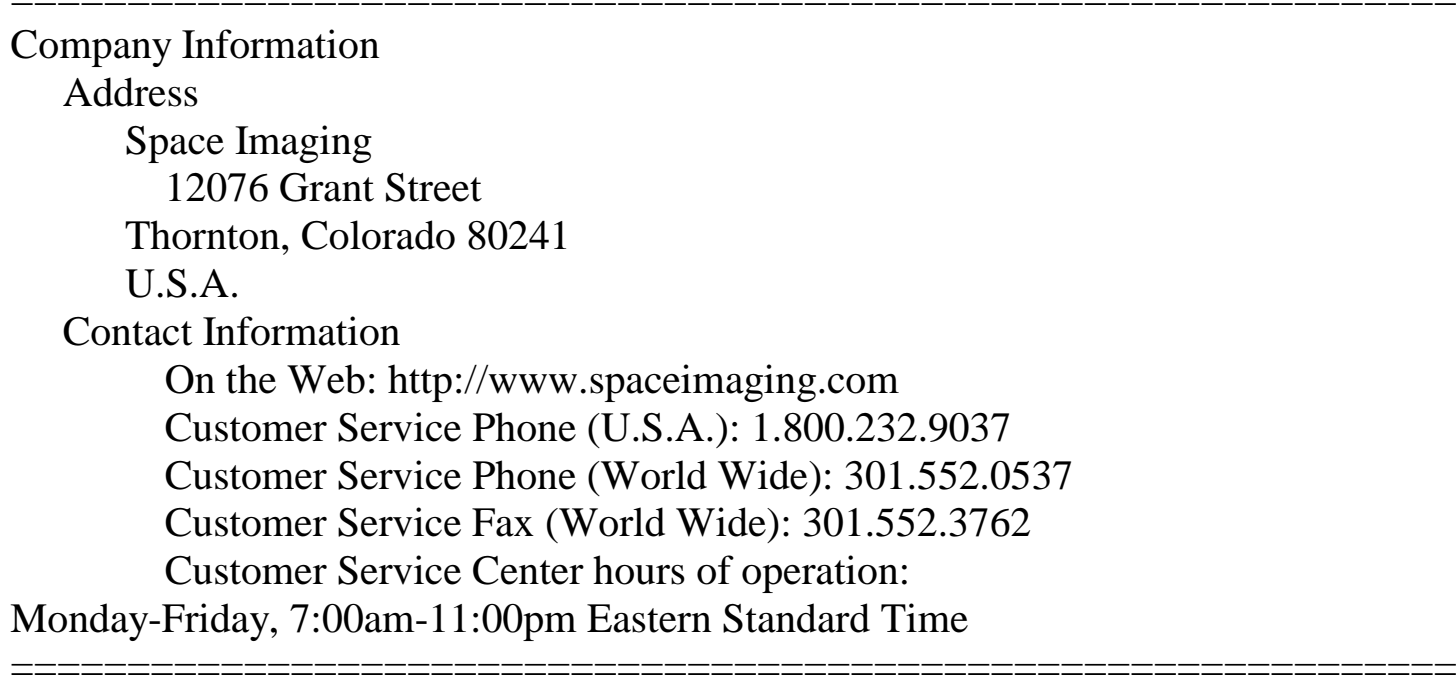

Product Order Metadata

Product Order Number: 34731

Customer Project Name: 262Korea

Product Order Area (Geographic Coordinates)

Number of Coordinates: 4

Coordinate: 1

Latitude: 37.54300000 degrees

Longitude: 128.27900000 degrees

Coordinate: 2

Latitude: 37.61100000 degrees

Longitude: 128.27900000 degrees

Coordinate: 3

Latitude: 37.61100000 degrees

Longitude: 128.40300000 degrees

Coordinate: 4

Latitude: 37.54300000 degrees

Longitude: 128.40300000 degrees

Product Order Area (Map Coordinates)

Coordinate: 1

Map X (Easting): 436364.78 meters

Map Y (Northing): 4162900.45 meters

Coordinate: 2

Map X (Easting): 447309.09 meters

Map Y (Northing): 4162823.63 meters

Coordinate: 3

Map X (Easting): 447261.15 meters

Map Y (Northing): 4155279.32 meters 
Coordinate: 4

Map X (Easting): 436306.88 meters

Map Y (Northing): 4155356.10 meters

Sensor Type: Satellite

Processing Level: Standard Geometrically Corrected

Image Type: PAN

Interpolator Method: Bicubic

Multispectral Algorithm: None

Stereo: Mono

Mosaic: No

Map Projection: Universal Transverse Mercator

UTM Specific Parameters

Hemisphere: $\mathrm{N}$

Zone Number: 52

Datum: WGS84

Product Order Pixel Size: 1.00 meters

MTFC Applied: Yes

DRA Applied: No

Media: CD

File Format: GeoTIFF

TIFF Tiled: No

Bits per Pixel per Band: 11 bits per pixel

Special Instructions: NA

Source Image Metadata

Number of Source Images: 1

Source Image ID: 2000042401463320000011612401

Product Image ID: 000

Sensor: IKONOS-2

Acquired Nominal GSD

Cross Scan: 1.02 meters

Along Scan: 1.06 meters

Scan Direction: 0 degrees

Nominal Collection Azimuth: 140.1404 degrees

Nominal Collection Elevation: 57.51640 degrees

Sun Angle Azimuth: 131.5839 degrees

Sun Angle Elevation: 57.10895 degrees

Acquisition Date/Time: 2000-04-24 01:46

Product Space Metadata

Number of Image Tiles: 1

$X$ Tiles: 1

Y Tiles: 1

Product MBR Geographic Coordinates

Number of Coordinates: 4

Coordinate: 1 
Latitude: 37.61099599 degrees

Longitude: 128.27834405 degrees

Coordinate: 2

Latitude: 37.61169248 degrees

Longitude: 128.40300342 degrees

Coordinate: 3

Latitude: 37.54298594 degrees

Longitude: 128.40355173 degrees

Coordinate: 4

Latitude: 37.54229116 degrees

Longitude: 128.27900683 degrees

Product Map Coordinates

UL Map X (Easting): 436306.88 meters

UL Map Y (Northing): 4162900.45 meters

Pixel Size X: 1.00 meters

Pixel Size Y: 1.00 meters

Columns: 11004 pixels

Rows: 7624 pixels

Product Component Metadata

Number of Components: 1

Tile ID: 0

Product Image ID: 000

Tile File Name: po_34731_pan_0000000.tif

Tile Geographic Corner Coordinates

Number of Coordinates: 4

Coordinate: 1

Latitude: 37.61099599 degrees

Longitude: 128.27834405 degrees

Coordinate: 2

Latitude: 37.61169248 degrees

Longitude: 128.40300342 degrees

Coordinate: 3

Latitude: 37.54298594 degrees

Longitude: 128.40355173 degrees

Coordinate: 4

Latitude: 37.54229116 degrees

Longitude: 128.27900683 degrees

Tile Map Coordinates

UL Map X (Easting): 436306.88 meters

UL Map Y (Northing): 4162900.45 meters

Pixel Size X: 1.00 meters

Pixel Size Y: 1.00 meters

Columns: 11004 pixels

Rows: 7624 pixels 


\subsection{Lagefehler mit unabhängigen Kontrollpunkten}

(a) Umprojektion

\begin{tabular}{|c|c|c|c|c|c|}
\hline ID & Xref & Yref & Xinp & Yinp & Dist \\
\hline 1 & 140633,70 & 456766,13 & 140659,02 & 456752,41 & 28,79 \\
\hline 2 & 145931,92 & 456780,91 & 146005,75 & 456753,47 & 78,76 \\
\hline 3 & 141951,50 & 455401,26 & 141969,65 & 455397,91 & 18,46 \\
\hline 4 & 137083,09 & 453456,53 & 136974,47 & 453564,09 & 152,87 \\
\hline 5 & 139861,14 & 450478,09 & 139880,13 & 450469,66 & 20,78 \\
\hline 6 & 137201,22 & 450082,56 & 137171,69 & 450146,91 & 70,80 \\
\hline 7 & 139933,92 & 451395,66 & 139944,47 & 451394,59 & 10,60 \\
\hline 8 & 140244,00 & 453891,06 & 140257,70 & 453872,06 & 23,43 \\
\hline 9 & 144484,27 & 454227,00 & 144539,11 & 454161,59 & 85,36 \\
\hline 10 & 143196,06 & 450101,56 & 143209,78 & 450085,75 & 20,93 \\
\hline 11 & 142448,30 & 453533,50 & 142424,03 & 453573,59 & 46,86 \\
\hline 12 & 146594,69 & 450538,75 & 146682,23 & 450443,84 & 129,12 \\
\hline 13 & 145870,12 & 451132,53 & 145940,78 & 451055,56 & 104,48 \\
\hline 14 & 144867,11 & 452500,47 & 144891,38 & 452488,88 & 26,89 \\
\hline 15 & 145775,10 & 454020,84 & 145847,97 & 453938,00 & 110,33 \\
\hline 16 & 144829,14 & 455607,59 & 144894,53 & 455550,63 & 86,73 \\
\hline 17 & 142926,06 & 456465,13 & 142967,83 & 456438,53 & 49,51 \\
\hline 18 & 143648,73 & 456627,44 & 143716,45 & 456584,41 & 80,23 \\
\hline 19 & 138495,89 & 456583,16 & 138467,42 & 456622,38 & 48,46 \\
\hline 20 & 141180,06 & 452515,94 & 141166,04 & 452530,87 & 20,48 \\
\hline
\end{tabular}


(b) Polynomtransformation

\begin{tabular}{|c|c|c|c|c|c|}
\hline ID & Xref & Yref & Xinp & Yinp & Dist \\
\hline 1 & 140633,70 & 456766,13 & 140627,15 & 456782,45 & 17,59 \\
\hline 2 & 145931,92 & 456780,91 & 145929,90 & 456792,51 & 11,78 \\
\hline 3 & 141951,50 & 455401,26 & 141933,14 & 455432,21 & 35,98 \\
\hline 4 & 137083,09 & 453456,53 & 137079,89 & 453453,48 & 4,42 \\
\hline 5 & 139861,14 & 450478,09 & 139868,10 & 450484,68 & 9,58 \\
\hline 6 & 137201,22 & 450082,56 & 137207,77 & 450085,26 & 7,08 \\
\hline 7 & 139933,92 & 451395,66 & 139933,29 & 451406,75 & 11,11 \\
\hline 8 & 140244,00 & 453891,06 & 140252,70 & 453883,61 & 11,45 \\
\hline 9 & 144484,27 & 454227,00 & 144496,89 & 454203,10 & 27,03 \\
\hline 10 & 143196,06 & 450101,56 & 143167,69 & 450145,51 & 52,31 \\
\hline 11 & 142448,30 & 453533,50 & 142391,58 & 453615,43 & 99,65 \\
\hline 12 & 146594,69 & 450538,75 & 146582,64 & 450547,38 & 14,82 \\
\hline 13 & 145870,12 & 451132,53 & 145870,10 & 451142,33 & 9,80 \\
\hline 14 & 144867,11 & 452500,47 & 144833,03 & 452535,84 & 49,12 \\
\hline 15 & 145775,10 & 454020,84 & 145807,29 & 453972,30 & 58,24 \\
\hline 16 & 144829,14 & 455607,59 & 144844,73 & 455586,70 & 26,07 \\
\hline 17 & 142926,06 & 456465,13 & 142901,16 & 456496,40 & 39,98 \\
\hline 18 & 143648,73 & 456627,44 & 143627,89 & 456649,88 & 30,63 \\
\hline 19 & 138495,89 & 456583,16 & 138527,24 & 456545,57 & 48,94 \\
\hline 20 & 141180,06 & 452515,94 & 141154,83 & 452565,96 & 56,02 \\
\hline
\end{tabular}


(c) Orthorektifizierung

\begin{tabular}{|c|c|c|c|c|c|}
\hline ID & Xref & Yref & Xinp & Yinp & Dist \\
\hline 1 & 140633,70 & 456766,13 & 140631,79 & 456770,06 & 4,38 \\
\hline 2 & 145931,92 & 456780,91 & 145930,74 & 456790,17 & 9,34 \\
\hline 3 & 141951,50 & 455401,26 & 141960,11 & 455403,63 & 8,93 \\
\hline 4 & 137083,09 & 453456,53 & 137074,95 & 453455,35 & 8,23 \\
\hline 5 & 139861,14 & 450478,09 & 139865,60 & 450484,02 & 7,42 \\
\hline 6 & 137201,22 & 450082,56 & 137203,67 & 450090,21 & 8,03 \\
\hline 7 & 139933,92 & 451395,66 & 139942,31 & 451394,77 & 8,43 \\
\hline 8 & 140244,00 & 453891,06 & 140245,30 & 453890,62 & 1,38 \\
\hline 9 & 144484,27 & 454227,00 & 144484,45 & 454213,67 & 13,33 \\
\hline 10 & 143196,06 & 450101,56 & 143192,57 & 450111,96 & 10,97 \\
\hline 11 & 142448,30 & 453533,50 & 142471,36 & 453524,78 & 24,66 \\
\hline 12 & 146594,69 & 450538,75 & 146584,13 & 450547,08 & 13,45 \\
\hline 13 & 145870,12 & 451132,53 & 145867,13 & 451146,43 & 14,22 \\
\hline 14 & 144867,11 & 452500,47 & 144860,20 & 452495,38 & 8,58 \\
\hline 15 & 145775,10 & 454020,84 & 145774,68 & 454007,83 & 13,02 \\
\hline 16 & 144829,14 & 455607,59 & 144818,29 & 455614,14 & 12,67 \\
\hline 17 & 142926,06 & 456465,13 & 142926,20 & 456464,95 & 0,23 \\
\hline 18 & 143648,73 & 456627,44 & 143652,44 & 456627,71 & 3,72 \\
\hline 19 & 138495,89 & 456583,16 & 138508,20 & 456571,62 & 16,87 \\
\hline 20 & 141180,06 & 452515,94 & 141181,61 & 452517,93 & 2,52 \\
\hline
\end{tabular}




\section{LEBENSLAUF}

Name Cho, Hyun-Kook

Geboren 28, 11, 1962 in Kangnung, Korea

als Sohn des Lehrer Kyu-hong Cho und seiner Ehefrau Hyuk-nyu Kweon

Familienstand Verheiratet seit 28, 04, 1991 mit Sun-cheon Kim, zwei Kind

$\begin{array}{ll}1969-1975 & \text { Grundschule " Jumunjin " } \\ 1975-1978 & \text { "Kangnung middleschool " } \\ 1978-1981 & \text { "Kangnung highschool" } \\ 1982-1989 & \text { "Department of Forestmanagement, College of Forestry, } \\ & \text { Kangweon National University" in Chuncheon/Korea } \\ & \text { Abschluß Bachelor of Agriculture } \\ & \text { "Department of Forestmanagement, Graduate school, } \\ & \text { Kangweon National University" in Chuncheon/Korea } \\ & \text { Abschluß Master of Agriculture } \\ & \text { Wehrdienst } \\ 1983-1991 & \text { Assistant bei Studiumberatung in College of Forestry, } \\ 1990-1992 & \text { Kangweon National University" in Chuncheon/Korea } \\ 1993-1994 & \text { Sprachkurs an der Universität Freiburg } \\ 1994-1996 & \text { Forstwissenschaftliche Fakultät an der Universität Freiburg } \\ 1997-2002 & \text { Institut für Forsteinrichtung und Ertragskunde } \\ & \text { Fakultät für Forstwissenschaften und Waldökologie } \\ & \text { Georg-August-Universität Göttingen }\end{array}$

